\title{
Blood Pressure Estimation using Oscillometric Pulse Morphology
}

\author{
By \\ Majid Mafi

\begin{abstract}
A Thesis
Presented to Faculty of Graduate Studies and Research In partial fulfillment of the Requirements for the degree of
\end{abstract}

Masters of Applied Science

Electrical and Computer Engineering University of Ottawa

(C) Majid Mafi, Ottawa, Canada, 2012 
I hereby declare that I am the sole author of this thesis. This is a true copy of the thesis, including any required final version, as accepted by examiners. Also, I authorize the University of Ottawa to lend this document to other institutions for the purpose of scholarly research.

Majid Mafi

I further authorize the University of Ottawa to reproduce this document by photocopying or by any other means, in total or in part, at the request of other institution or individuals for the purpose of research

Majid Mafi 


\section{Abstract}

This thesis work presents the analysis of Oscillometric blood pressure pulse waveform under different pressure points (Systolic, Mean Arterial, and Diastolic Pressures). Pulse waveform's characteristics were determined from the waveforms at three different pressures and are compared for subjects at three different age groups. Estimation of blood pressure using a morphology based approach was done by using the change of pulse waveform characteristics at different pressure points. Pulse waveform's characteristics that were obtained from pulse waveforms are utilized to estimate SBP, MAP, and DBP. The estimates obtained with pulse morphology based technique are compared with a BP measurement device and Maximum Amplitude Algorithm. Maximum slope of the pulse was also used for blood pressure estimation. The effect of movement and breathing on proposed method and MAA were compared and it was observed that breathing artifacts affect less the proposed method. 


\section{Acknowledgments}

It is my pleasure to thank the many people who made this research possible.

I would like to express my gratitude to my supervisors Dr. Voicu Groza and Dr. Miodrag Bolic for their support, guidance, and assistance with the research and the writing of this thesis.

I would also like to thank Biosign Technology Inc., Ontario Centres of Excellence (OCE), and Wisense for their collaborative research support and funding.

The work could also not have been completed without the support and aid of fellow research team members including Professor Dr. Hilmi Dajani and research collaborators Dr. Izmail Batkin and Dr. Sreeraman Rajan. I would also like to thank and acknowledge postdoctoral fellows Dr. Saif Ahmad and Dr. Soojeong Lee and my lab mates Mohamad Forouzanfar, Karen Soueidan, and Silu Chen. It has been a pleasure collaborating with each member of the team.

Last but not least, I am heartily thankful to my parents for their understanding, patience, and encouragement for the period of my graduate studies. 


\section{Table of Contents}

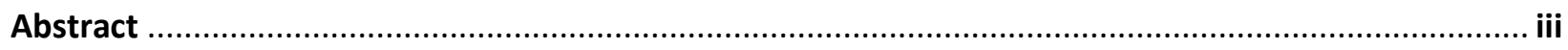

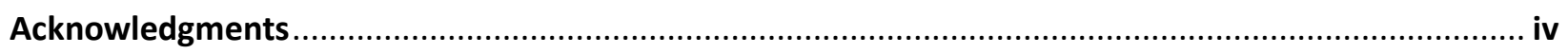

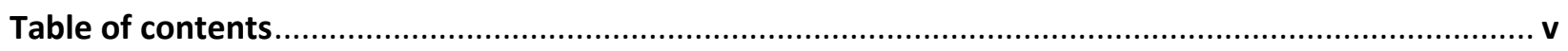

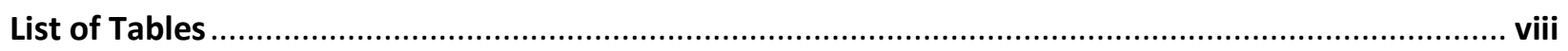

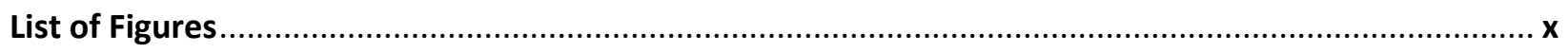

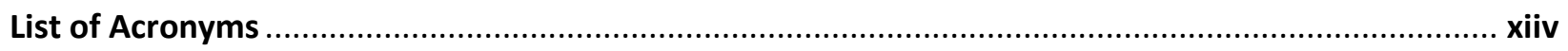

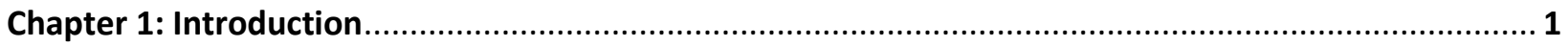

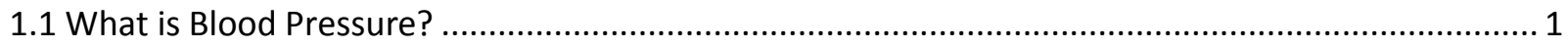

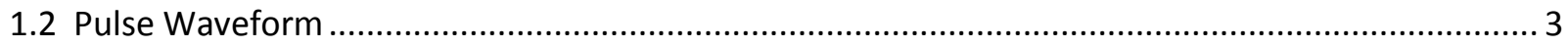

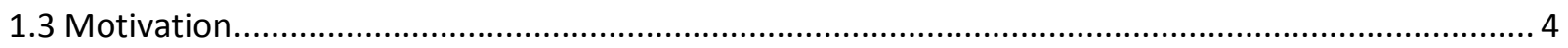

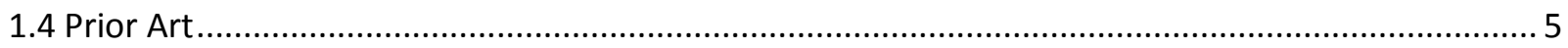

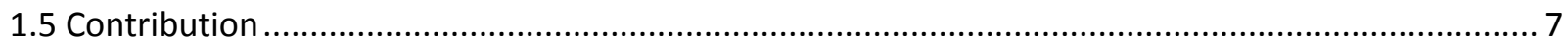

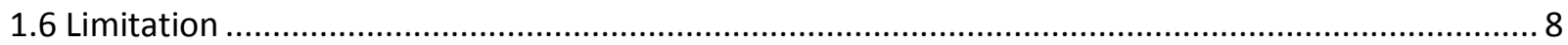

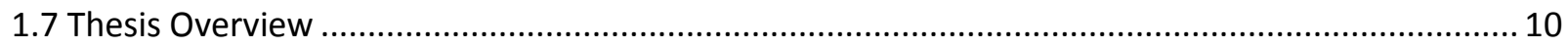

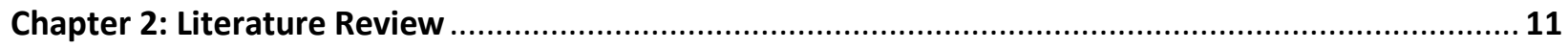

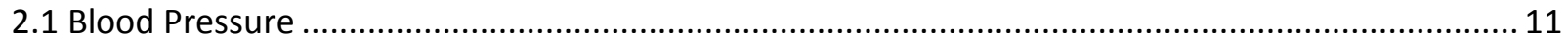

2.2 Methods of Blood Pressure Measurements ............................................................................. 12

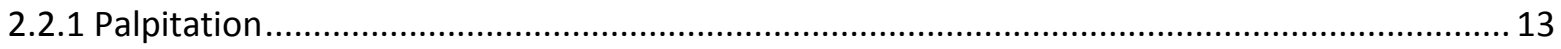

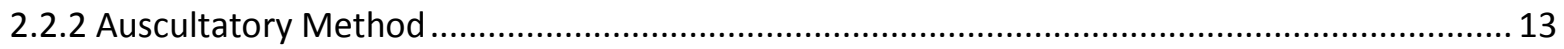

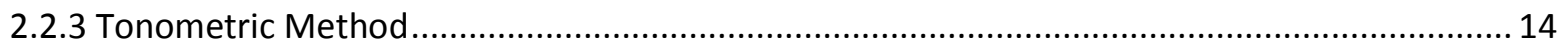

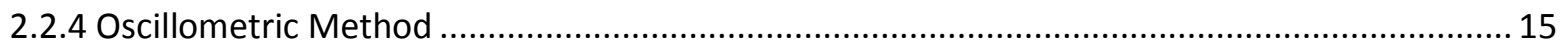

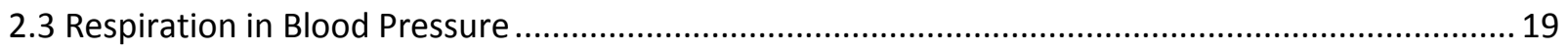

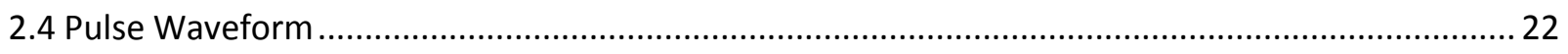

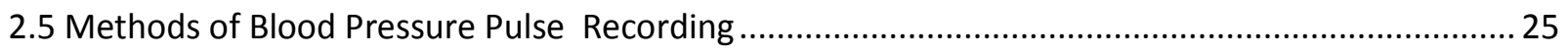

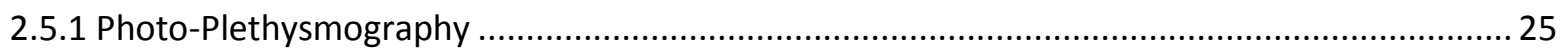




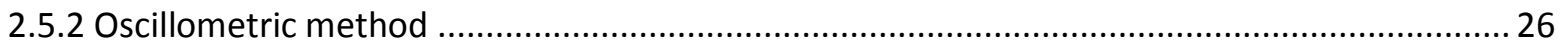

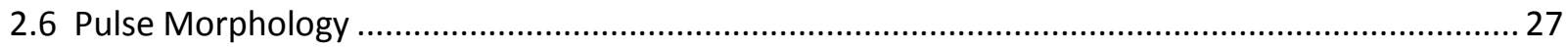

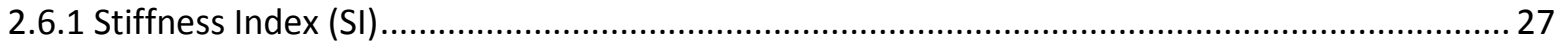

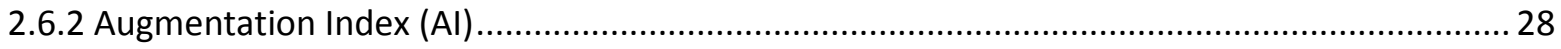

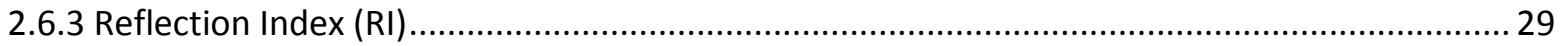

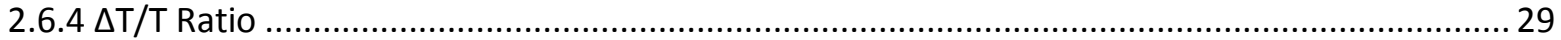

2.6.5 Time Parameters of Blood Pressure Pulse Waveform ........................................................... 29

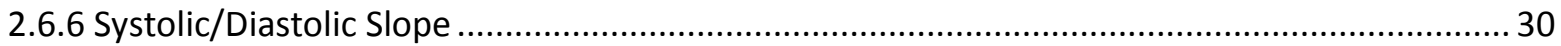

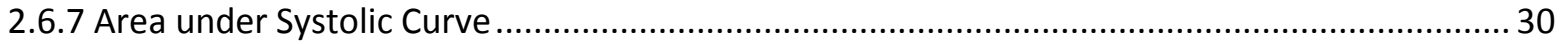

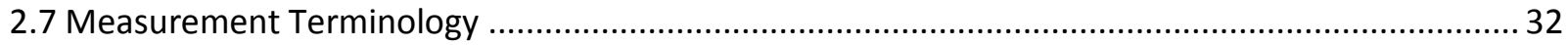

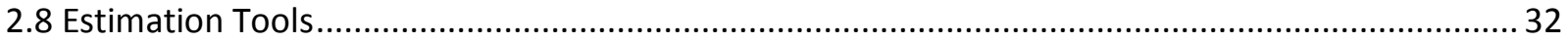

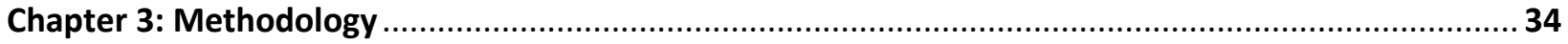

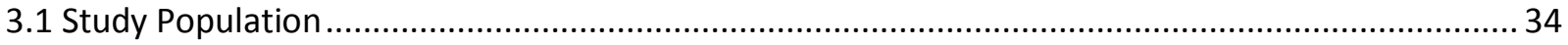

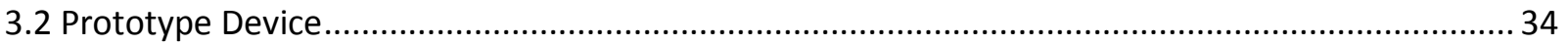

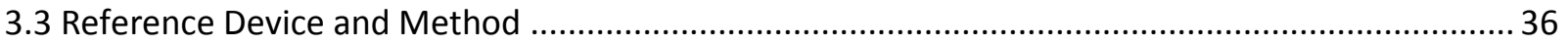

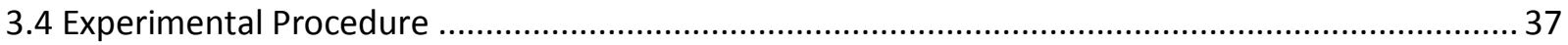

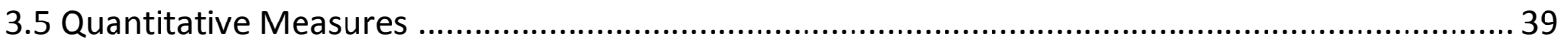

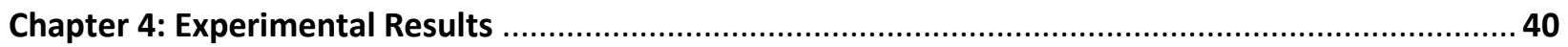

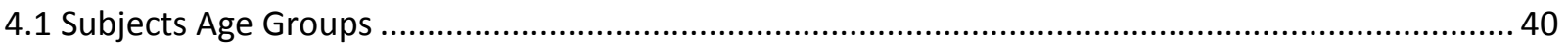

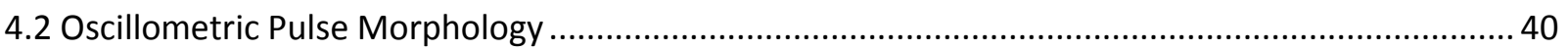

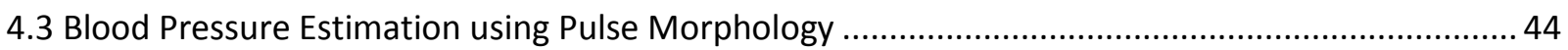

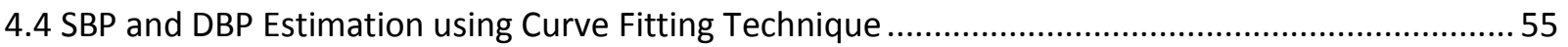

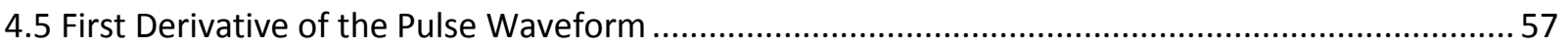

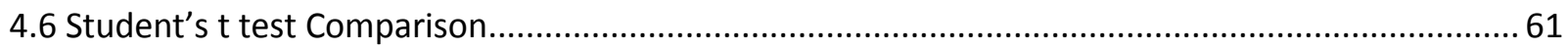

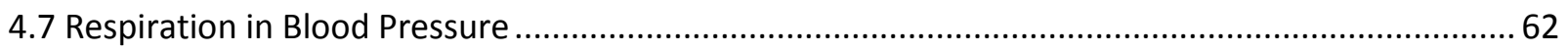

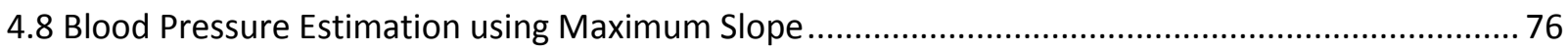




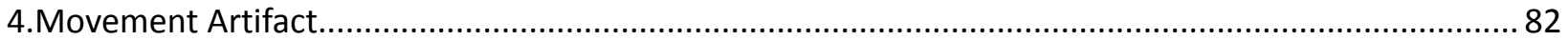

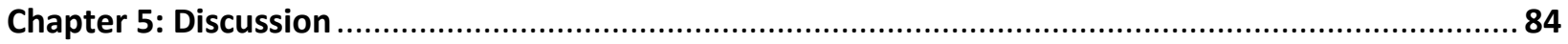

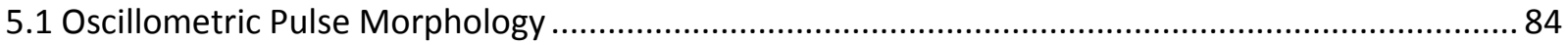

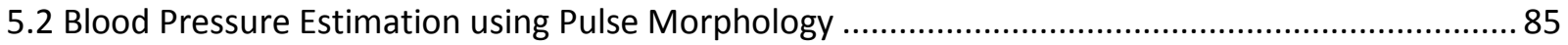

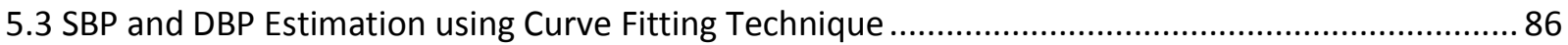

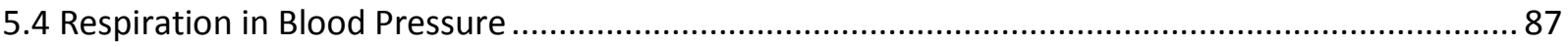

5.5 Blood Pressure Estimation using Maximum Slope …..................................................................... 89

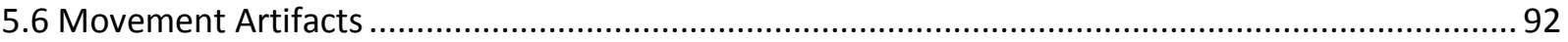

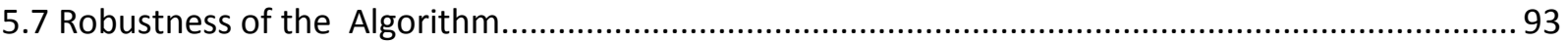

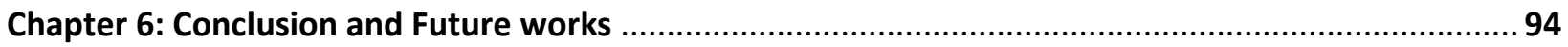

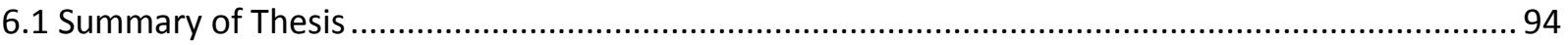

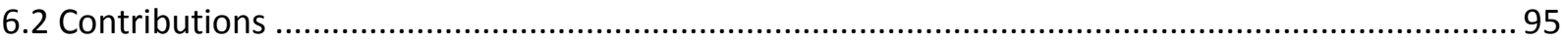

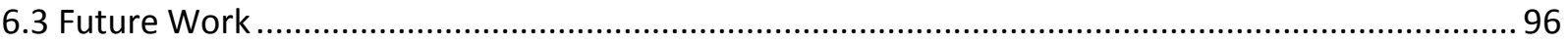

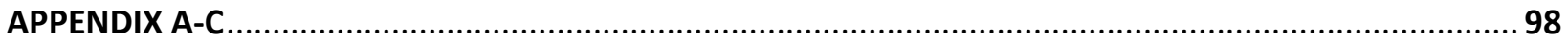

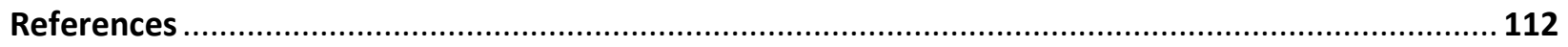




\section{List of Tables}

Table 2.1: Common usage of pulse parameters

Table 3.1: Experimental steps

Table 4.1: Reflection Index

Table 4.2: Augmentation Index

Table 4.3: Stiffness Index

Table 4.4: $\Delta \mathrm{T} / \mathrm{T}$ ratio 42

Table 4.5: Crest Time

Table 4.6: Dicrotic Wave Time

Table 4.7: Relative Crest Time 43

Table 4.8: Relative Dicrotic Wave Time .43

Table 4.9: Time Delay Parameter .43

Table 4.10: Area under Systolic Curve .44

Table 4.11: Mean Absolute Error (mmHg) with OMRON results at SBP 49

Table 4.12: Mean Absolute Error (mmHg) with OMRON results at MAP .49

Table 4.13: Mean Absolute Error (mmHg) with OMRON results at DBP 50

Table 4.14: Comparison of the presented method with MAA and OMRON results at SBP 50

Table 4.15: Comparison of the presented method with MAA and OMRON results at MAP ....... 51

Table 4.16: Comparison of the presented method with MAA and OMRON results at DBP 51 
Table 4.17: Systolic Blood Pressure Student's t test

Table 4.18: Mean Arterial Pressure Student's t test

Table 4.19: Diastolic Blood Pressure Student's t test 62

Table 4.20: Comparison of Max. Slope method with MAA and OMRON results in SBP 79

Table 4.21: Comparison of Max. Slope method with MAA and OMRON results in MAP 79

Table 4.22: Comparison of Max. Slope method with MAA and OMRON results in DBP 80

Table 4.23: Maximum Slope Student's t test 80

Table 4.26: Blood Pressure Estimation with Movement 82 


\section{List of Figures}

Figure 2.1: Circulatory System with arrows representing the Blood Circulation into the Body ... 11

Figure 2.2: Auscultatory Blood Pressure Measurement method 14

Figure 2.3: Example of Cuff Deflation Waveform 17

Figure 2.4: Cuff Deflation Waveform and Oscillometric Waveform 18

Figure 2.5: Maximum Amplitude Algorithm 20

Figure 2.6: Blood Pressure Waveform formation 23

Figure 2.7: Blood Pressure Pulse Waveform at different Ages 24

Figure 2.8: Oscillometric Blood Pressure Waveforms 27

Figure 2.9: Blood Pressure Pulse Parameters 31

Figure 3.1: Block Diagram of BP Prototype Development 35

Figure 3.2: Reference results using OMRON recording and MAA method 36

Figure 3.3: 5 Trials of Oscillometric Recording using the Prototype Device 38

Figure 4.1: Sample Pulses at different Pressure Points for Subjects from different Age Groups 41

Figure 4.2: Plot of Reflection Index .46

Figure 4.3: Plot of Stiffness Index .47

Figure 4.4: Plot of Augmentation Index 47

Figure 4.5: Plot of $\Delta \mathrm{T} / \mathrm{T}$ Ratio 48

Figure 4.6: Different Pressure range in a Pulse .49 
Figure 4.7: Bland Altman plot comparing the presented method with OMRON results in SBP . 52

Figure 4.8: Bland Altman plot comparing the presented method with OMRON results in MAP 53

Figure 4.9: Bland Altman plot comparing the presented method with OMRON results in DBP 54

Figure 4.10: Smoothed Reflection Index Plot and First Derivative .55

Figure 4.11: Smoothed Augmentation Index Plot and First Derivative 56

Figure 4.12: Smoothed $\Delta \mathrm{T} / \mathrm{T}$ ratio Plot and First Derivative 56

Figure 4.13: Smoothed Stiffness Index Plot and First Derivative .57

Figure 4.14: Bland Altman plot comparing the SBP Estimated from curve fitted graphs with OMRON results .58

Figure 4.15: Bland Altman plot comparing the DBP Estimated from curve fitted graphs with

OMRON results .59

Figure 4.16: First Derivative of the Blood Pressure Pulse Waveform 60

Figure 4.17: Cuff Deflation Waveform with Fast Breathing .64

Figure 4.18: Pulses at Systolic region with Fast Breathing 64

Figure 4.19: Pulses at MAP region with Fast Breathing 65

Figure 4.20: Pulses at Diastolic region with Fast Breathing 65

Figure 4.21: Comparison of Proposed Method with Reference Results under Fast Breathing at SBP .66

Figure 4.22: Comparison of Proposed Method with Reference Results under Fast Breathing at MAP 
Figure 4.23: Comparison of the Proposed Method with Reference Results under Fast Breathing at DBP

Figure 4.24: Comparison of MAA with Reference Results under Fast Breathing at SBP, MAP, and DBP

Figure 4.25: Cuff Deflation Waveform with Deep Breathing .70

Figure 4.26: Pulses at Systolic region with Deep Breathing 70

Figure 4.27: Pulses at MAP region with Deep Breathing 71

Figure 4.28: Pulses at Diastolic region with Deep Breathing 71

Figure 4.29: Comparison of Presented Method with Reference Results under Deep Breathing at SBP .72

Figure 4.30: Comparison of Proposed Method with Reference Results under Deep Breathing at MAP .73

Figure 4.31: Comparison of Presented Method with Reference Results under Deep Breathing at DBP. .74

Figure 4.32: Comparison of MAA with Reference Results under Deep Breathing at SBP, MAP, and DBP .75

Figure 4.33: Plot of Maximum Slope of all Pulses .78

Figure 4.34: The Curve Fitted of Maximum Slope plot 78

Figure 4.35: BP Estimation from Maximum Slope method .79 
Figure 4.36: Bland Altman plot comparing the Maximum Slope method with OMRON results in SBP 80

Figure 4.37: Bland Altman plot comparing the Maximum Slope method with OMRON results in MAP 81

Figure 4.38: Bland Altman plot comparing the Maximum Slope method with OMRON results in DBP 81

Figure 4.39: Cuff Deflation Pressure Waveforms with Movement during the Recording. 83

Figure 4.40: Pulses with Movement of Arm during the Recording 83

Figure 5.1: Blood Pressure Estimation using MAA with Fast Breathing...... 87

Figure 5.2: Blood Pressure Estimation using MAA with Deep Breathing 88

Figure 5.3: Blood Pressure Estimation using four parameters of Pulse Waveforms with Fast Breathing . 90

Figure 5.4: Blood Pressure Estimation using four parameters of Pulse Waveforms with Deep Breathing .91 


\section{List of Acronyms}

AI: Augmentation Index- A pressure ratio in $\mathrm{mmHg}$ and is defined as the difference of the systolic peak (F) and diastolic peak (P) over systolic peak of pulse waveform.

BP: Blood Pressure- Measure of the pressure exerted by the blood in the arteries during one cardiac cycle.

DBP: Diastolic Blood Pressure- Minimum pressure produced in the arteries that happens at the end of each cardiac cycle.

$\boldsymbol{E C G}$ : Electrocardiogram- Non-invasive recording of the electrical activity of the heart.

$\boldsymbol{F W}$ : forward-going pressure wave- Wave produced by flow of the blood into the arteries from left ventricle to the periphery side during systole phase of the heart.

mmHg: millimeter mercury- Commonly used unit adopted in blood pressure to measure pressure.

MAA: Maximum Amplitude Algorithm- Height based algorithm to estimate blood pressure in oscillometry that is based on characteristic ratios.

MAP: Mean Arterial Pressure- Average arterial blood pressure of a person during a cardiac cycle.

OMW: Oscillometric Waveform

PPG: Photoplethysmogram- Non-invasive recording of blood pressure pulse waveform obtained from the tissue pads attached to the ears, fingers, and toes where there is a high degree of superficial vasculature.

$\boldsymbol{P W V}$ : Pulse Wave Velocity- The velocity of the pulse traveling through the arteries from the left ventricle to the periphery region.

$\boldsymbol{R} \boldsymbol{W}$ : Reflected Pressure Wave- Wave which reflected from periphery side back to the heart in the arteries during the diastole phase of the heart.

$\boldsymbol{R I}$ : Reflection Index- An index to show the pressure reflected from the periphery side back to the arteries around the heart during diastole phase of the heart.

SBP: Systolic Blood Pressure- Maximum pressure produced in the arteries that happens at the beginning of each cardiac cycle.

SI: Stiffness Index- An index characterizing features of the contour of pulse that are determined by PWV in the aorta and large arteries and by the stiffness of the arteries 


\section{Chapter 1: Introduction}

\subsection{What is Blood Pressure?}

Cardiovascular diseases are one the main cause of death and studies showed that one third of deaths of the world's population are due to the heart diseases [1]. Blood pressure (BP) is one of the most common parameter to measure in clinical practice which can provide significant information about physiological condition of the subjects and the heart functioning. BP is the pressure of blood circulation exerted upon the wall of arteries during the cardiac cycle and it is usually measured in millimeter mercury $(\mathrm{mmHg})$. Systolic Blood Pressure (SBP) and Diastolic Blood Pressure (DBP) are the values estimated in blood pressure measurement. Physiologically, SBP happens in the beginning of the cardiac cycle (systole phase) when the heart contracts, which increase the pressure of the arteries around the heart to the maximum level and push the blood from the left ventricle of the heart to the vessels. On the other hand, DBP happens at the end of the cardiac cycle (diastole phase) when the heart relaxes, which drop the pressure of the arteries to the minimum level and the blood fills the chambers of the heart [2] [3].

Blood pressure estimating can be either Invasive (direct) or Noninvasive (indirect). Intra- arterial measurement is an invasive method which considers being the most accurate method of blood pressure measurement, However, the invasive method has its own disadvantages such as danger of potential infection and bleeding and requires close supervision [4]-[8].

Noninvasive methods are most commonly used because they are safer and require less training. Palpitation, Auscultatory, and Oscillometric are some examples of noninvasive methods. In Auscultatory method a sphygmomanometer and a stethoscope are needed. This method is 
considered to be the gold standard of noninvasive methods since the manometer does not require any calibration over time [10] [11]. A proper size pressure cuff which is connected to the manometer is inflated above the expected SBP and deflated slowly. A stethoscope is placed at the brachial artery (elbow) while the pressure of cuff is reducing. An observer would mark the pressures from the manometer when the Korotkoff sounds representing SBP and DBP are heard [9] [10]. However, some studies showed that Auscultatory method seems to underestimate SBP by $5-20 \mathrm{mmHg}$ and overestimate DBP by $12-20 \mathrm{mmHg}[11]$.

Oscillometric is the most popular method in BP devices [12]. This method was invented by Marey on 1876, by observing the oscillation in the sphygmomanometer in Auscultatory method which is caused by the oscillation of blood flow in the artery under the cuff [12]. The procedure is very similar to Auscultatory method. A cuff is inflated above the expected SBP and deflated slowly under expected DBP. A pressure sensor or transducer is within the cuff to measure the pressure of the cuff during deflation and a waveform which is called cuff deflation pressure waveform can be provided [12] [13]. The pulsation in subject's arteries is reflected on cuff deflation pressure waveform and from these oscillations different algorithms have been proposed to estimate SBP and SBP [12].

Blood pressure values fluctuate over time [2]. Factors such as gender, age, and height can affect the values as well [5] [9]. In children, the normal ranges of BP are usually lower than for adults. As adults age, SBP tends to rise and DBP tends to fall. In the elderly, BP tends to be above the normal adult range because of reduced flexibility of the arteries. In females the average ranges of BP are lower than males. Also, individual's BP can vary with exercise, stress, emotional reactions, sleep, digestion, and time of day [2] [5] [9] [12].A large part of Blood Pressure fluctuation may be due to respiration, or breathing [77], that was first discovered by Hales [68]. 
Breathing in normal depth may cause SBP fluctuation up to 3-6 mmHg and deep breathing has been found to influence SBP up to $15-20 \mathrm{mmHg}$ [71].

\subsection{Pulse Waveform}

Pulse waveform is the graphical representation of BP and the shape of the waveform is a result of complex interaction between the left ventricle of the heart and blood circulation system [14]. Pulse waveform which is a pressure signal usually contains more information than is captured by BP values and most of this information can be extracted from the pulse morphology, which refers to the shape and characteristics of the pulse waveform over a span of one cardiac cycle [15] [16]. Pulse contour analysis, has produced new methods of estimating clinically relevant parameters [15]. Pulse contour varies in different part of the circulation and it also depends on physiological and pathological condition of the organisms [16].

Pulse waveform can also be obtained from invasive and noninvasive methods. In invasive method, a needle which goes in the blood circulation system connects to an electronic pressure transducer. The pressure sensor constantly monitors the pressure beat by beat and provides a graph of pressure against time for every single heartbeat. The provided waveform for every beat is called pulse waveform [17].

Photoplethysmogram (PPG) and Oscillometric method are examples of noninvasive methods of recording pulse waveform. PPG expresses changes in the volume of blood in a fingertip, which provides a mean of determining properties of the vascular tree during the cardiac cycle [18]. The signals can be obtained from the tissue pads of the ears, fingers, and toes where there is a high degree of superficial vasculature [19]. In Oscillometric method, oscillations or pulses can be observed from cuff deflation pressure waveform. The advantage of Oscillometric method over 
PPG is that in Oscillometric method pulse waveforms can be obtained and studied at different pressure points while in PPG pulses are obtained at a constant pressure. Although, pulses extracted from Oscillometric recording are more sensitive to artifacts such as breathing and movement than PPG pulses [18] [19].

\subsection{Motivation}

Pulse morphology is description and analysis of pulses waveforms based on their parameters and characteristics. In Blood Pressure studies, pulse morphology is mostly used to provide information about cardiovascular system. Studies regarding to pulse morphology showed mixed results about characteristics of pulse waveforms and changes of pulse waveform parameters due to the age and cardiovascular diseases. [18] [21]-[23]. Some focus on the changes of the pulse contour characteristics based on the increase of large arteries stiffness by age [18] [21] [23] and some compare the pulse shape in elderly and hypertensive subjects [22], whereas other have shown the characteristics of pulses captured by PPG method at different parts of vasculature tree [20] [24].

Pulse morphology can provide significant information about the cardiovascular system and heart functioning. From literature review, different methods to determine the characteristics of the pulses have been proposed to predict the cardiovascular disease [21]. Pulse waveforms change their shape and characteristics with age due to the increase of large arteries stiffness. [14] [18] [20] [21] [23] study the effect of age on pulse waveform and provide physiological and biological explanations for these changes. Since the shape and characteristics of pulse waveform change by age, little effort has been put to come up with some standards of pulse waveform for every age group to predict any abnormalities which can lead to further diseases or disabilities. 
However, in this thesis work, the characteristics of the pulses at different pressure points will be observed and compared for every subject. The changes of the characteristics of the pulse waveform with age will be also studied at different pressure points.

In previous works, pulse waveforms were mostly observed from photoplethysmogram method which provides the pulse waveforms at constant pressure while pulses could have different shape and characteristics at different pressure points for every individual [25] [26]. Therefore, pulses were detected from Oscillometric method in this thesis work to observe and study the pulses at different pressure regions.

Since pulses change their shape and characteristics at different pressure regions of cuff deflation pressure waveform, a new method of estimation BP based on the morphology of pulse waveform is proposed. The proposed method of BP estimation may be used to check the other methods of estimating blood pressure. Meanwhile, characteristics of the pulses obtained at different pressure points in this method can provide a good estimate about the cardiovascular functioning and health of the subjects.

\subsection{Prior Art}

Prior work in blood pressure pulse morphology is scattered around a variety of works. After recording the pulse waveforms, characteristics of the pulses were obtained by considering the amplitude of two peaks of the signal (pressure parameters) and time parameters of the pulses. Most of the pulse characteristics can be obtained from three main parameters which are needed to be extracted from each pulse and are listed as follows:

1. Existence of dicrotic notch

2. Amplitude of the peaks or pressure parameters in $\mathrm{mmHg}$ 


\section{Time parameters of each peak}

- Peak locations and time difference between peaks locations

Pulse Wave Velocity (PWV) was known as a good indicator of arterial stiffness and has been regarded as a marker reflecting vascular damage [27]-[29]. PWV is the velocity of the pulse traveling through the arteries from the left ventricle to the periphery region and it can be recorded from non-invasive devices (i.e. tonometry, cuff pressure) and used to evaluate arterial stiffness [30]. [28] [31] [32] have shown that PWV can be used as a prognostic predictor in patients with hypertension or end-stage renal failure. Many studies compared the difference of pulse contour in different age groups and explained why the shape varies throughout the age and how changes in stiffness of arteries can affect the pulse waveform [18] [20] - [22] [23] [24]. [33] - [37] compare the pulse waveform between healthy subjects and hypertensive subjects. It is also noticed that most of cardiovascular diseases have affected the shape and characteristics of the pulse waveform and these effects can be observed from the early stages of the diseases using pulse morphology.

Time parameters and slope information of pulses from PPG can change by age and arterial stiffness and they can be related to blood pressures, especially DBP [38] [39]. In stiff arteries, pulses will travel faster to the periphery, therefore the PWV increases due to the low distensibility or high stiffness of the arteries [40] [41]. An artery with low distensibility has much less elastic behavior than a normal artery [74].

Pressure ratios can be evaluated based on the amplitude of the peaks which also vary by age and cardiovascular diseases. They can provide important information about the arteries condition and heart functioning [42]. Also, the pressure ratios can determine left ventricle work-load and can 
be used an important independent predictor of mortality, in normotensive and hypertensive individuals [36] [43] - [45].

Some studies compare the Electrocardiogram (ECG) with pulse waveform over the time to see the relations between the changes in ECG and Pulse waveform. Compared with ECG, pulse waveforms have less complexity and can be easily acquired. Therefore, cardiovascular abnormalities can be viewed from the pulses instead of ECG [46].

\subsection{Contributions}

The main contributes of this thesis work are as the follow:

- Analyzing the characteristics of the pulse waveforms at different pressure points for every individual and comparing the characteristics for different age groups

- Developing an new method of blood pressure estimation using parameters of the all the pulses detected from the cuff deflation pressure waveform

- Checking the robustness of the proposed method under breathing and movement artifacts and comparing it to MAA and BP values estimated with OMRON device

- Developing a new method of estimating blood pressure using the maximum slope of the pulse waveforms determined from the first derivative of each pulse waveform.

Pulse waveforms can be extracted either with invasive or non-invasive methods. The contribution of the work performed here is to first extract pulses from Oscillometric measurement. First, all pulses will be extracted from cuff deflation waveforms and the characteristics of the pulses will be studied at different pressure points. The characteristics of the pulses change by going from systolic region toward diastolic region. By dividing the subjects to 
different age groups, it was observed that the characteristics of the pulses at SBP, MAP and DBP are almost the same for subjects with same age. The comparison of the characteristics of Oscillometric pulses at different pressure points and different age groups was firstly done in this project. The work in Sections 4.2 and 5.1 have led to a publication in IEEE International Symposium on Medical Measurements and Applications [64].

Different methods to estimate SBP and DBP from Oscillometric recording exist. Another contribution is to develop a new method of BP estimation using the morphology of the pulses extracted from the cuff deflation waveform derived from Oscillometric recording. Although, the characteristics of the pulses change with age, but the change of these characteristics from systolic region to diastolic region is same for all the healthy subjects from all the age groups. The results in this study led to a publication in International Conference of the IEEE Engineering in Medicine and Biology Society [65], which also includes Sections 4.3 and 5.2.

In addition, a method of BP estimation using the maximum slope of the pulse waveforms has developed. Section 4.8 in chapter 4 and Section 5.5 in chapter 5 provide the results of BP estimation using this method. The proposed method of BP estimation using morphology of the pulse waveform was tested under breathing and movement artifacts and the effects of breathing and movement on the proposed method, MAA, and BP values estimated by OMRON device were compared. Sections $4.8,5.4$, and 5.6 provide the results related to this topic.

\subsection{Limitation}

Blood pressure estimation algorithm using Oscillometric pulse morphology is validated by comparison with reference values recorded by OMRON HEM-790 ITCAN Blood pressure 
measurement device. According to SP10 standard, there was around one minute delay between the OMRON reading and Oscillometric recoding [47].

A limitation can be the time delay between the Oscillometric recording and using OMRON device to obtain reference values. The Oscillometric recording was taken after a minute from the reference reading. However, it is known that blood pressure fluctuates over time [48]. Therefore, the time delay between the two methods can create some error and the subject's blood pressure can differ in the seconds of measurement. The reason for this delay is due the safety standards given by SP-10 during the BP measurement [67]. Ideally, the reference reading (OMRON reading) and Oscillometric reading should occur at the same time to avoid any limitation due to the fluctuation of BP over time, but this is not possible for the safety of the subjects.

In Blood pressure measurement, Intra- Arterial measurement which is an invasive method is considered to be the gold standard of BP estimation and Auscultatory method is the most accurate method of BP estimation in noninvasive methods [4] [13]. Non-invasive measurement results should be considered as reference values while in this case OMRON reading and Maximum Amplitude Algorithm (MAA) results were used as the references blood pressure to compare our estimations which may be another source of error.

In Oscillometric pulse morphology, every single pulse in cuff deflation pressure waveform comes into the consideration and any movement of the subject during the recording can affect the shape and characteristics of the pulses and cause an error.

The presented method has been tested on 18 healthy subjects only. It is needed to be applied on subjects with different health conditions for better validation. Also, testing the method on more subjects can help for more accurate conclusion. 
Blood pressure changes at various times of days with various factors [48]. Factors such as digestion, emotional reactions can also affect the results. In this thesis work, these factors did not take into the consideration. However, they would provide some sources of error.

\subsection{Thesis Overview}

In this thesis the characteristics of Oscillometric pulse waveforms at different age groups and different pressure points is obtained and studied. Also, from the parameters of the pressure waveforms, a new method of estimating blood pressure is proposed. Chapter 2 presents the current work and literature review about blood pressure and different methods of blood pressure measurement, blood pressure pulse waveform and method of recording it, and introduced characteristics of the pulse waveforms and effect of age and cardiovascular diseases on them. Chapter 3 presents the methodology of the proposed method. Chapter 4 shows the result of the proposed algorithm on a data set of 18 subjects. Chapter 5 provided the result and discussion for the work on Oscillometric pulse morphology and the algorithm used based on morphology to estimate blood pressure. Chapter 6 will end with the summarized contribution of the thesis, conclusion, and propose some potential future work in this topic. 


\section{Chapter 2: Literature Review}

\subsection{Blood Pressure}

BP is the pressure of the circulating of the blood upon the walls of blood vessels. Arterial blood pressure changes during the course of the cardiac cycle [2]. Systolic Blood Pressure (SBP) refers to the highest pressure in the beginning of the cardiac cycle when heart contracts or is in systole phase. On the other hand Diastolic Blood Pressure (DBP) refers the minimum pressure at the end of cardiac cycle when the heart relaxes or is in the diastole phase (Figure 2.1) [2] [3]. BP is one of the most common parameters in clinical practice and can provide significant information about the physiological condition of the subjects. However, factors such as sleeping, stress, physical activities, and different time of day can influence the BP results [48].

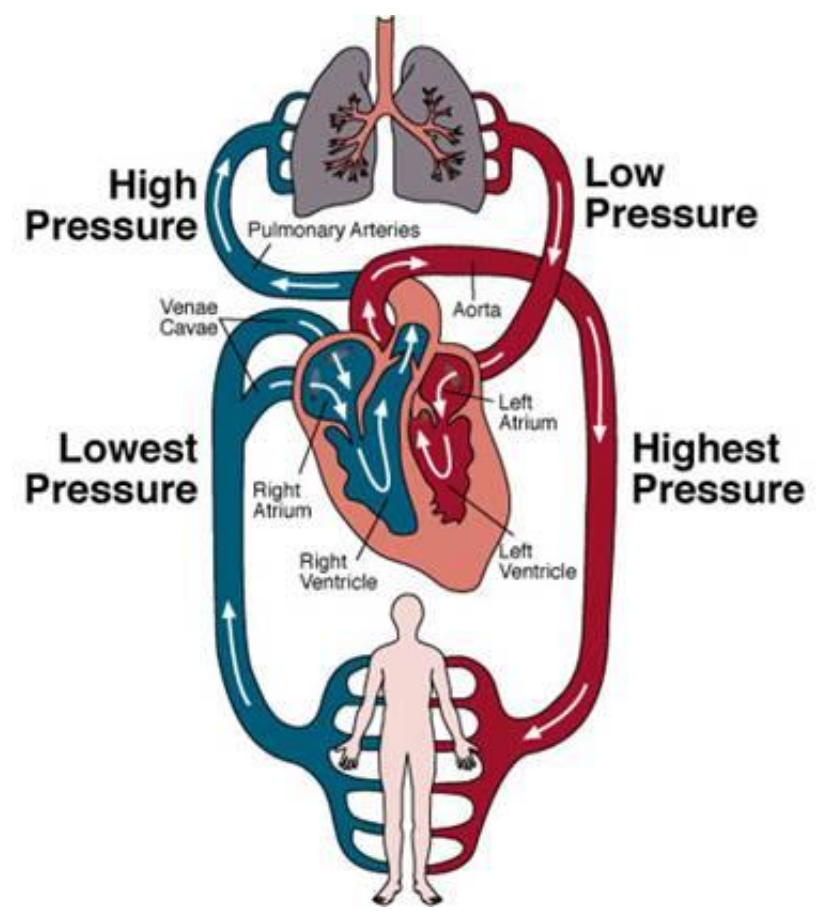

Figure 2.1: Circulatory System with the arrows representing the Blood Circulation into the Body (SOURCE: Revision World. Retrieved 12 23, 2011, from the Circulatory system: http://www.revisionworld.com/country.php) 


\subsection{Methods of Blood Pressure Measurement}

The most accurate method of blood pressure is invasive or direct using an arterial line [4]. A cannula needle is inserted into the artery, into the fluid system, and connected to a pressure sensor or transducer. It continuously records the BP over long period of time. It measures the pressure beat-by-beat and displays a graph of pressure against the time. Therefore, the fluctuation of BP over time can be observed. Invasive method is usually employed in human and veterinary intensive care medicine, anesthesiology, and for research purposes. Invasive pressure monitoring is infrequently associated with complications such potential infection and bleeding. Therefore, patients with this method of arterial monitoring require a very close supervision. This method is usually reserved for patients with rapid variation in their arterial pressure [4]-[7] [10].

Non-invasive methods of blood pressure measurement have fewer complications than invasive methods. Especially in home care monitoring, non-invasive devices have become increasingly popular. Automatic measurement features and easiness of use have also contributed to their growing of popularity. Nevertheless, the accuracy of these devices has not reached to the necessary level yet, and only few of them are clinically validated and have similar estimate with Auscultatory method which is used by doctors [49].

Noninvasive blood pressure measurement methods are indirect and based mainly on measuring counter-pressure which is an external pressure such cuff pressure. An overview of some different noninvasive methods will be given with a description of each. Blood pressure units is millimetermercury $(\mathrm{mmHg})$, which although is not an SI unit, is nonetheless a globally approved blood pressure unit for historical reasons [49]. 


\subsubsection{Palpitation}

Palpitation method measures the blood pressure by putting the hand or an inflatable cuff on an arterial line. The blood pressure can be estimated by counting the number of the pulses sensed from the artery over a period of time. This method estimate SBP only and DBP cannot be measured from palpitation. Some studies have shown that palpitation method overestimate SBP. Therefore American Heart Association (AHA) recommends that palpitation be used to get an estimate of SBP before using any other blood pressure method. Palpitation method is most often used in emergency situation [50].

\subsubsection{Auscultatory Method}

Auscultatory method is considered to be the most accurate and gold standard of non-invasive blood pressure measurements method [75]. The method was first presented by Nikolai Korotkoff (1905) in his M.D. thesis to measure animals' blood pressure by using a Rivo-Rocci sleeve, mercury manometer and child stethoscope [49].

This method uses a smooth proper size inflatable cuff, a stethoscope, and a sphygmomanometer to measure the blood pressure. Sphygmomanometer is a blood pressure meter to measure the pressure of the inflatable cuff and will give an absolute result without need for calibration over time [10] [11] (Figure 2.2). The cuff will be inflated quickly by squeezing a rubber bulb above the expected systolic pressure until the artery is completely occluded. The stethoscope will be placed on brachial artery at the elbow. The cuff pressure will be slowly released. At this point no sound is heard by stethoscope. When blood starts to flow in the artery, the turbulent flow creates a pounding sound which is called the first Korotkoff sound. The pressure at which this sound is heard is the SBP and can be read from sphygmomanometer. After systolic pressure, as the cuff 
pressure reduces the sound will be muted and another sound will be heard at DBP. The appearance and disappearance of sound can be used to determine systolic and diastolic blood pressures [9] - [13].

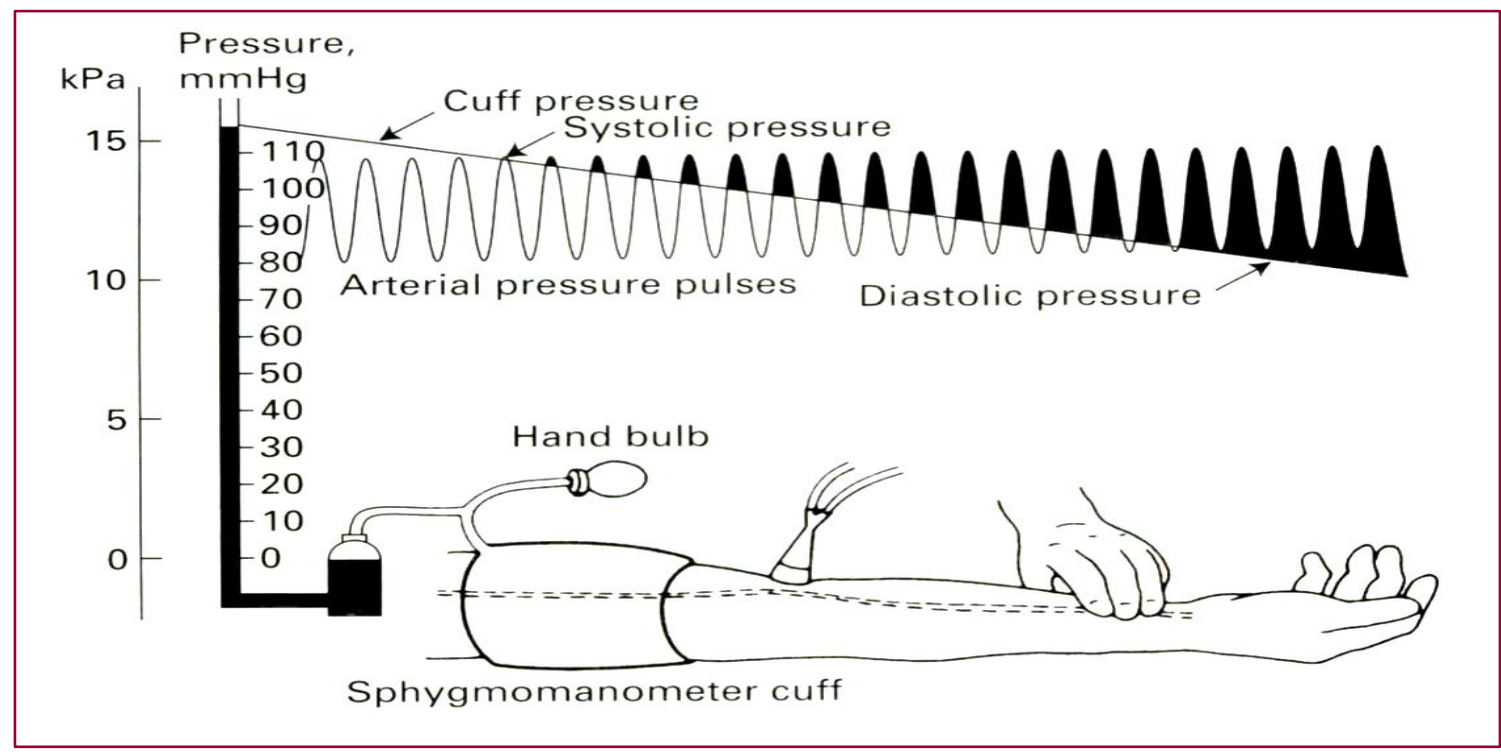

Figure 2.2: Auscultatory Blood Pressure Measurement method (SOURCE: Nichols \& O'Rourke, "McDonald's Blood Flow in Arteries", $4^{\text {th }}$ Edition, Page 132, 1998 [52])

Auscultatory methods needs high degree of training for reading the pressures by hearing the

Korotkoff sounds and it is not recommended for home monitoring care since it is not very practical. In ambulatory measurements, when the subject is able to move moderately, noise may become dominant and make the measurement less accurate. This can be avoided by using two identical stethoscopes under the cuff, one located on the upper side, and one located on the distal side. In this way, noise reaches both stethoscopes at the same time, but the blood pressure pulse propagating through the brachial artery arrives after a time delay [10].

\subsubsection{Tonometric Method}

Tonometric method was first presented by Pressman and Newgard in 1963. The arterial tonometer is a pressure measurement system that can non-invasively and continuously record the 
pressure alteration in a superficial artery with sufficient bone support, such as radial artery. It uses a small transducer, or a rigid sensor array, or a flexible diaphragm. They can be attached on the skin above the pulsating artery. Skin and tissue located between the sensor and array transfer pressure pulsations between them. When the pulsations reach their strongest level, the sensor is regarded as being correctly positioned. This can be facilitated by using a sensor array and selecting sensor elements with the strongest amplitude. Then, the sensor or sensor array is pushed toward the vessel using air pressure. The vessel flattens when the pressure and the force against the artery wall increases. Arterial pressure in the top of the flattened artery's center equals the supporting pressure, allowing the recording of reasonable accurate blood pressure [53]. If the pressure increases too much, the artery will occlude and the measurement will be spoiled [54].

\subsubsection{Oscillometric Method}

Oscillometric method was first introduced by Marey in 1876 [12] [66]. At that time, the arm was placed within a rigid chamber that was made airtight. The air could be pumped into the chamber and provide a uniform external pressure to the arm. Marey noticed that the pressure within the chamber fluctuated with the pulse. He associated the amplitude of these fluctuations at different external pressures to indicate when external pressure is equal to the arterial pressure. He also found out that at this pressure, the arterial wall stress is zero [66]. Oscillometric method considers being the most popular method in most of electronic devices [49]. Oscillometric monitors are easy and simple to use and there is no need of training or supervision during the measurement. The procedure is very similar to Auscultatory method. A pressure sensor or transducer is within an inflatable cuff which is placed around the arm or wrist. The cuff is inflated rapidly around $20-30 \mathrm{mmHg}$ above the SBP and deflated slowly below the DBP at a rate 
of 2 to $3 \mathrm{mmHg}$ per second. As the cuff deflates the pressure sensor records the pressure inside the cuff electronically and presents a signal which is known as cuff deflation pressure waveform (Figure 2.3) [12] [13] [56].

The Oscillometric method of measuring blood pressure uses the amplitude of cuff pressure oscillation to estimate SBP and DBP. However, in Oscillometric method another pressure, known as Mean Arterial Pressure (MAP) is estimating. The pressure sensor detects the amplitude of the oscillations impinged on the cuff by brachial artery pulses. As the pressure of the cuff decreases, the amplitude of the oscillation increases, until they reach a peak and, then they decrease. The cuff pressure at which the maximum oscillation appears corresponds to the MAP [55] [56].

Unlike Auscultatory method, MAP is being found first in oscillometric method and SBP and DBP are extracted from MAP. MAP is defined as the average arterial pressure of an individual. For intra-arterial pressure (invasive method), MAP corresponds to the average of the total arterial pressure during one cardiac cycle [60]. It is been tested that at MAP, external pressure (cuff pressure) is equal to the internal pressure (arterial pressure). At this time, the arterial wall contains zero stress and the vessel is minimally distended. Because arterial pressure varies with time, the artery is unloaded, on average, when cuff pressure is equal to MAP. Also, the arterial compliance is at maximum level (when the transmural pressure is zero). Transmural pressure is the difference of arterial pressure and cuff pressure. Arterial compliance (C) is the change in arterial blood volume $(\Delta \mathrm{V})$ due to a given change in arterial blood pressure $(\Delta \mathrm{P})$, i.e., $\mathrm{C}=\Delta \mathrm{V} / \Delta \mathrm{P}$ [76]. The arterial volume pulse will be at maximum when the transmural pressure is zero, which occur when MAP is equal to cuff pressure [66]. MAP is not commonly estimate on clinical practice and other methods of blood pressure measurements and usually is not presented on the 
screen of blood pressure monitor devices. From the SBP and DBP, the MAP can approximately calculate by [55]:

$$
M A P=D B P+\frac{1}{3} \cdot(S B P-D B P)
$$

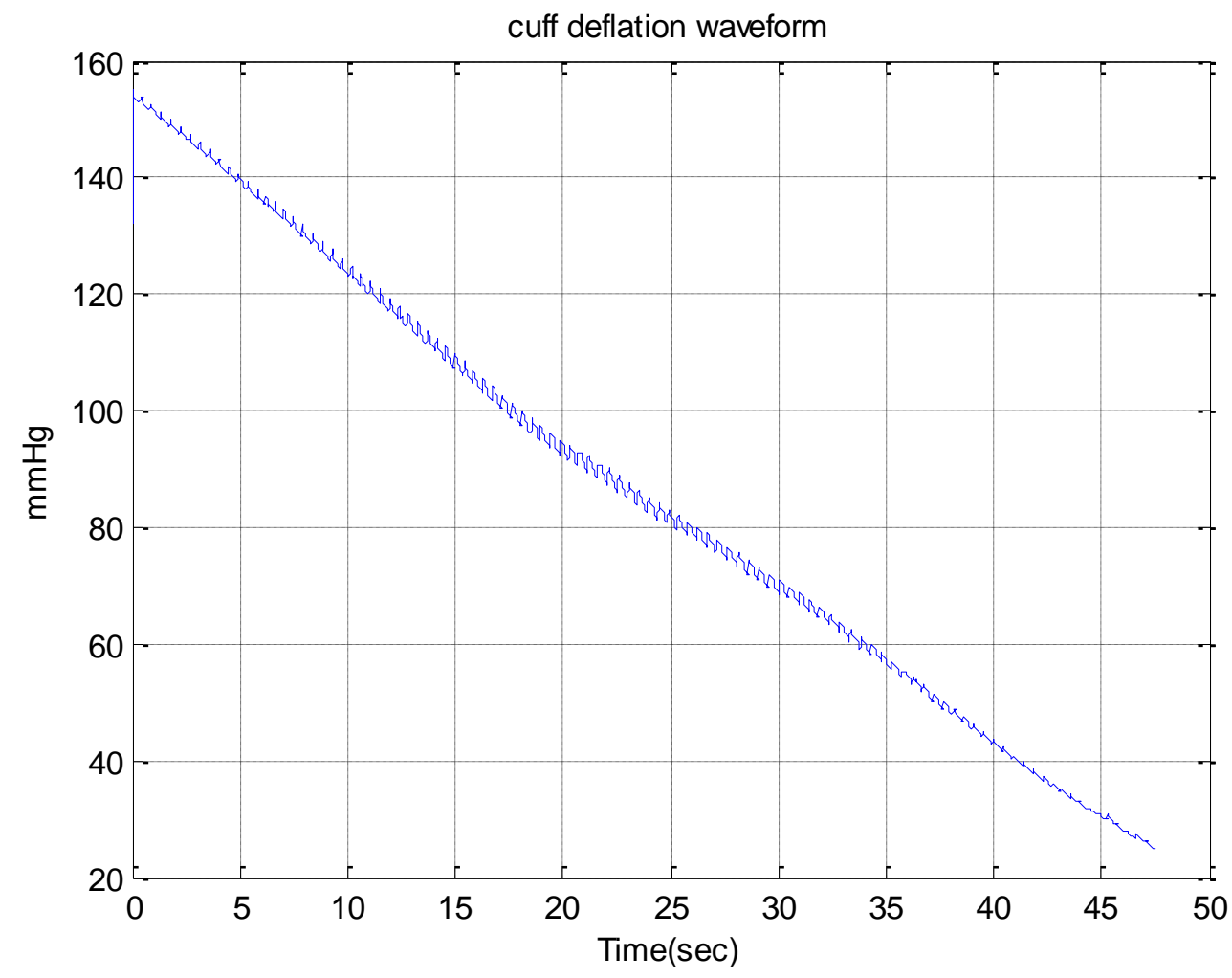

Figure 2.3: Example of Cuff Deflation Waveform

The following observation can be obtained from Oscillometric waveform during the blood pressure measurement:

1. Cuff oscillation increases until it reaches the maximum amplitude, then it decreases with cuff pressure

2. Maximum oscillation correlates with cuff pressure which is equal to MAP.

3. Oscillations in cuff pressure waveform are usually in the range of 1 to $5 \mathrm{mmHg}$ [66]. 
The oscillation or Oscillometric pulses can be extracted from cuff deflation pressure waveform and presented a signal knows as Oscillometric waveform (OMW) [57]. Filtering and deterending are used to extract OMW from cuff deflation pressure waveform [55] [58] [59]. Filtering is used to remove the measurement noise and the frequency components of the deflating cuff pressure. Pulses on OMW have different amplitude and detecting the peaks of the pulses is the main key in the Oscillometric method. The point with highest amplitude on OMW is Oscillometric Pulse Index (OPI) which represents MAP point and by mapping the point to cuff deflation pressure waveform the corresponding pressure can be determined (Figure 2.4) [52] [55] [62]:

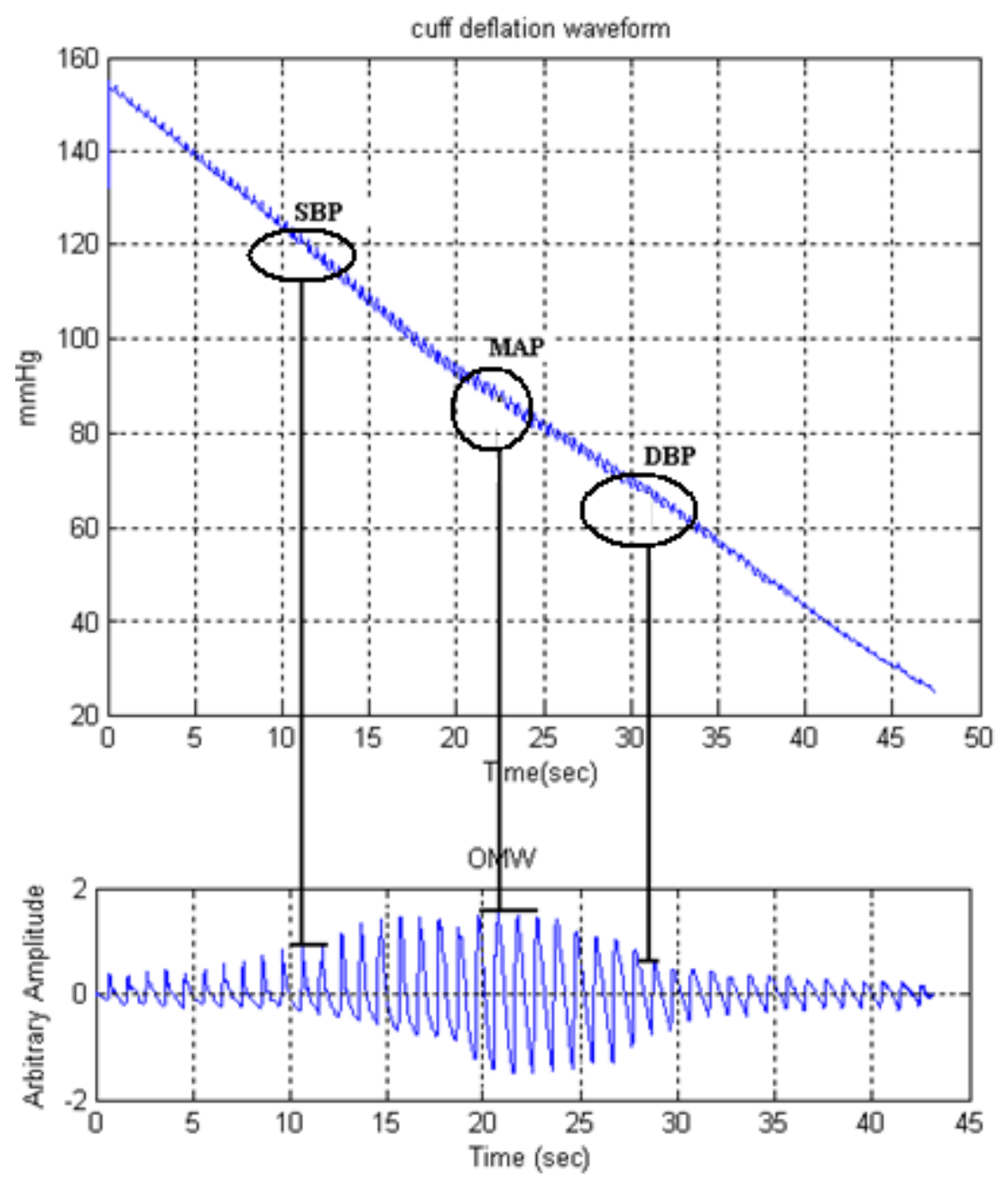

Figure 2.4: Cuff Deflation Waveform and Oscillometric Waveform 
OPI divides OMW into two different regions: the left side is called systolic region and the right side of OPI is called diastolic region. The name systolic region and diastolic region do not hold much physical meaning, except to indicate the location where the points for SBP and DBP may lie. The systolic and diastolic points are typically determined by ratios relative to the MAP point and by mapping systolic and diastolic points to cuff deflation pressure waveform, SBP and DBP can be estimated [52] [55] [61] [62].

The Maximum Amplitude Algorithm (MAA) is the most popular Oscillometric algorithm for determining blood pressure [9] [55]. This algorithm operates first by finding the point on the envelope which corresponds to MAP (OPI). Systolic and diastolic points can be found by multiplying the OPI by systolic and diastolic ratios respectively. The systolic and diastolic ratios are not fixed and may differ from a device to another, but may range from 0.45 to 0.73 for systolic and 0.69 to 0.83 for diastolic ratios. Since these points are defined, they can be mapped to cuff deflation pressure waveform to find the corresponding pressures (Figure 2.5) [55] [62].

In this thesis, a new method of estimating SBP, MAP, and DBP is presented based on the characteristic of the Oscillometric pulses extracted from cuff deflation pressure waveform and is compared to the existing Oscillometric algorithms of BP estimation to validate the proposed method.

\subsection{Respiration in Blood Pressure}

Breathing at different levels can have large influences on BP estimation [68]. In some cases, the breathing effects can be large enough that estimated BP values cannot be truly representative of the subject's actual blood pressure. 


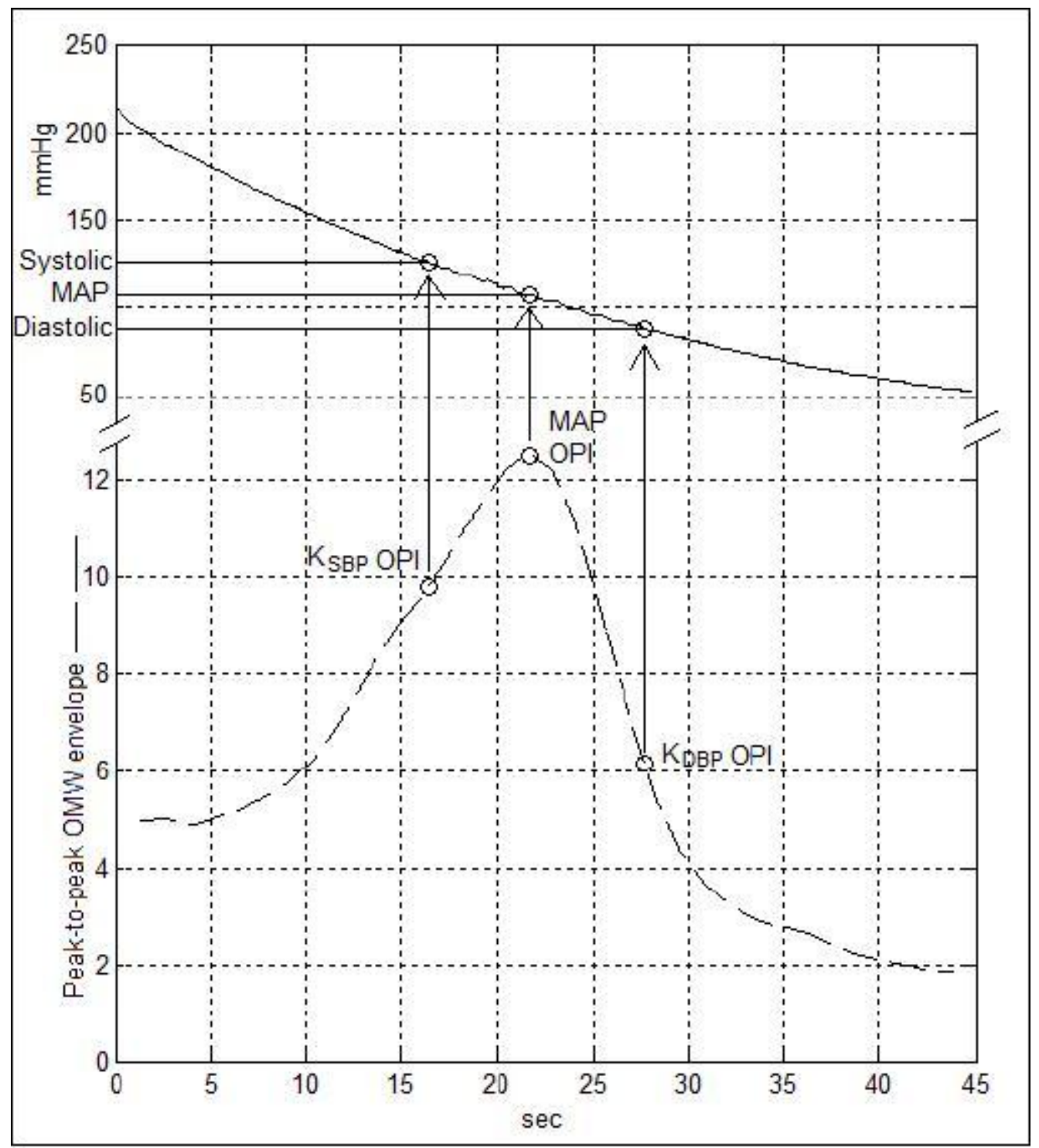

Figure 2.5: Maximum Amplitude Algorithm

(SOURCE: S. Chen, V. Z. Groza, M. Bolic, H. R. Dajani, “Assessment of Algorithms for Oscillometric Blood Pressure Measurement", International Instrumentation and Measurement Technology Conference, pp. 1763-1767, 2009 [62])

Stephan Hales was the first to discover the effect of breathing on heart rate and blood pressure oscillation [68]. It has been discovered that most effects of breathing that influence BP is through Amplitude, Frequency Modulation, and additive effects [69] [70]. Deep breathing has been found to influence SBP up to $15-20 \mathrm{mmHg}$ and breathing in normal depth can cause SBP fluctuation up to 3-6 mmHg [71]. This fluctuation is large enough and can be the motivation to 
develop techniques to remove the respiration effects from BP or develop new method of BP estimations which are less sensitive to respiration.

As respiration occurs, gas is transferred in and out of the lungs flowing from areas with high pressure to areas with low pressure [2]. During inspiration, the diaphragm and intercostal muscles expands the thorax cavity which houses the lunges, heart and ribs. Therefore, the pressure in thorax which is called intra-thoracic pressure drops below the atmospheric pressure and allows air to move into the lungs. During exhalation opposite of this behavior occurs.

Cardiac output is the volume of the blood pumped into the arteries from the heart per minute and is directly related to blood pressure through stroke volume. Stroke volume is the total volume of the blood pumped out of the heart per heartbeat. As cardiac output increases, more blood will pumped out of the heart which results in an increase in Blood Pressure. Therefore, BP can be modeled as the cardiac output multiplied by the resistance to blood flow by the blood vessels [72]. The interaction between the cardiac output and intra-thoracic pressure is the result in the amplitude changes in BP.

During inspiration, the thorax expands as intra-thoracic pressure reduces. Therefore, there is more room for heart to fill a larger capacity with blood. Thus, cardiac output increases and BP will rise as well. During exhalation, intra-thoracic pressure is high and thorax cavity contracts. As a result, the capacity of the heart is less to be filled with blood. Therefore, cardiac output decreases and BP reduces as well [72].

Frequency Modulation is influenced by changes in the heart rate of the subject. Heart rate is controlled by the autonomous nervous system which is a part of nervous system that acts as an involuntary control system for certain functions such as heart rate and blood pressure [73]. Two 
motor neurons namely, vagal and sympathetic are responsible for controlling the heart rate using innervating the cardiac sinoarterial (SA) node of the heart. The excitation of these two neurons depends on respiration phase. Increase and decrease in $\mathrm{CO}_{2}$ concentration, with respect to the inspiration and expiration phase of respiration, excite and inhibit these neurons. In inspiration, the vagal neuron is inhibited and the sympathetic neuron is excited which leads to a higher heart rate. In expiration, vagal neuron is excited and sympathetic is inhibited, leading to lower heart rate. This cyclic behavior influences the Frequency Modulation and can be observed in the pulse to pulse intervals in blood pressure [73].

\subsection{Pulse Waveform}

Blood pressure waveform can be a graphical representation of the BP over one cardiac cycle. The shape of the blood pressure waveform is the result of complex interaction between left ventricle of the heart and the systemic circulation. The pulse waveform consists of two peaks: An

early systolic peak and a second peak or point of inflection that occurs a short time after the first peak in early diastole (Figure 2.6). The first peak is formed by the pressure transmitted along a path from the left ventricle to the periphery and the second peak is formed by pressure transmitted along the aorta and large arteries to sites of impedance mismatch in the lower body, where is reflected back up to the aorta [14].

The shape of the pulse contour is result of two pressure waves: forward-going pressure wave $(\mathrm{FW})$ and reflected pressure wave $(\mathrm{RW})$. Forward going pressure wave is transmitted along a direct path from the left ventricle of the heart to the periphery (i.e. fingers). During the systole phase, the heart contracts and the pressure of the arteries around the heart rises to the maximum level. The pressure around the heart is much larger than the pressure in the periphery, therefore 
the blood will flow from upper part of the body to the lower limb. The first peak is the result increasing the pressure during systole and transmitting the pressure to the lower limb. During diastole phase, the heart relaxes and the pressure drops to the minimum level. During diastolic the pressure of the periphery is more than the pressure of the arteries around the heart, therefore the blood flows toward the heart. The pressure will be transmitted from small vessels in the periphery to the aorta around the heart and creates the second peak of the blood pressure pulse waveform (RW). The time difference between two peaks can be used to infer the transit time taken for the pressure to propagate along the aorta and large arteries to the major sites of reflection in the lower body and back to the root of the arteries around the heart (Figure 2.6) [14].

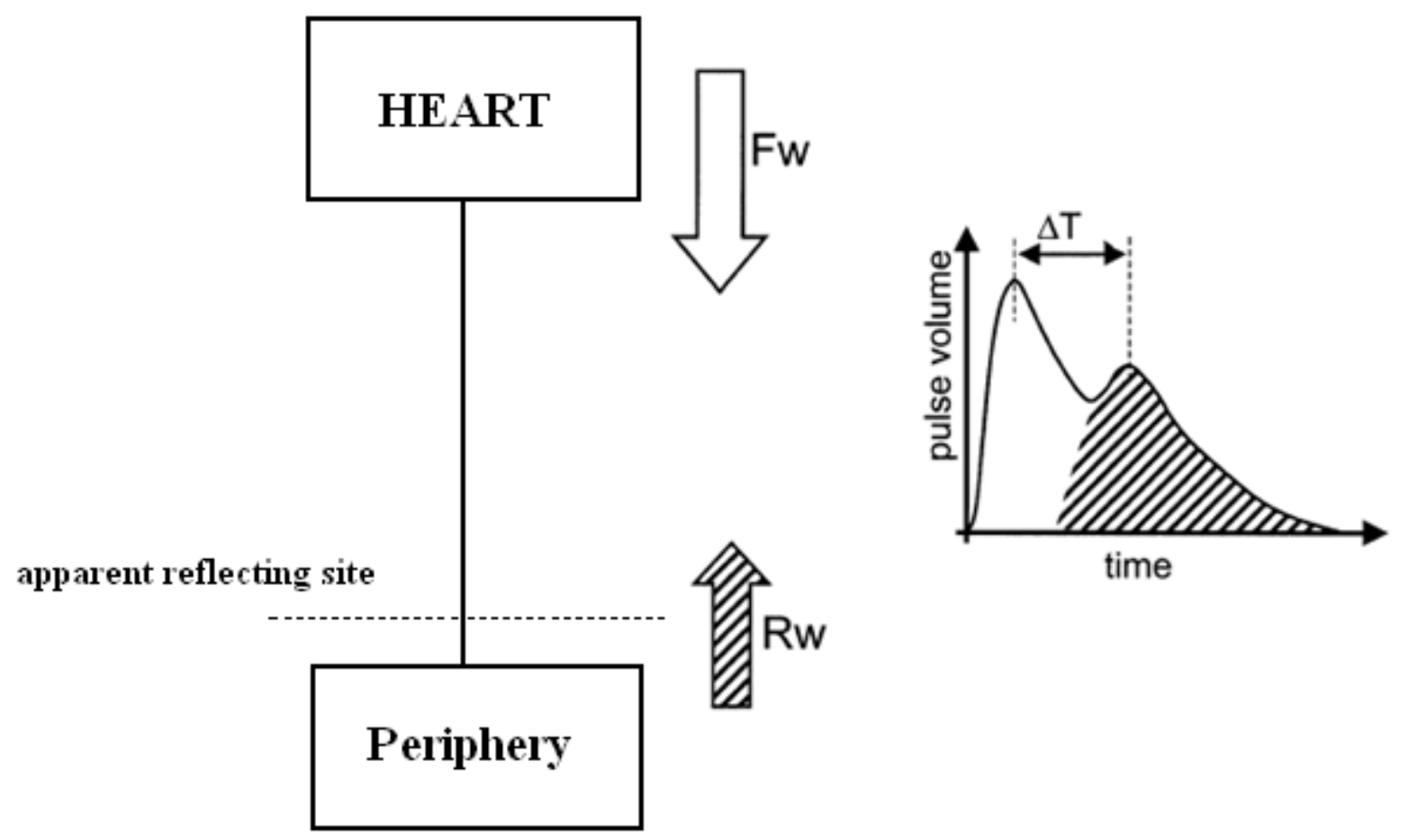

Figure 2.6: Blood Pressure Waveform formation

(Based on: S.C. Millasseau, R. P. Kelly, J. M. Ritter, and P. J. Chowienczyk, "Determination of age-related increase in large arteries stiffness by digital pulse contour analysis”, Clinical Sience, Vol. 103, pp. 371-377, 2002 [14] )

The pulse contour varies in the different parts of the circulation cycle and it depends on the physiological and pathological condition of the organism [16]. Arterial stiffness is an important factor affecting the shape of the pulse contour; also it is an important determinant of 
cardiovascular risks [35]. Changing of blood pressure contour at different level of arterial tree is due to the difference in vessels compliance at different part of the circulation [35] [52]. Aging is accompanied by increase of large arteries stiffness [23] [34] [35] [37] although other factors such as posture, exercise, and cardiovascular diseases can affect the shape and characteristics of the pulse waveform [35] [37]. Pulse Wave Velocity (PWV) was known as a good indicator of arterial stiffness [27]-[29]. The change in pulse contour can be result of increase of stiffness in large arteries with an increase in Pulse Wave Velocity (PWV). Increase in PWV can decrease the time taken for pressure wave reflected (RW) from the periphery region (lower limb) to return to the aorta and arteries around the heart in upper limb. Therefore, RW arrives earlier in the cardiac cycle and that causes the dicrotic notch to become less visible [23] [52]. Also, the first peak of the pulse contour is the result of FW and left ventricular work load. Therefore, the shape of the contour correlates with the degree of the left ventricular hypertrophy, in normotensive and hypertensive individuals [43]. Example of changes of blood pressure contour at different ages is given in Figure 2.7:

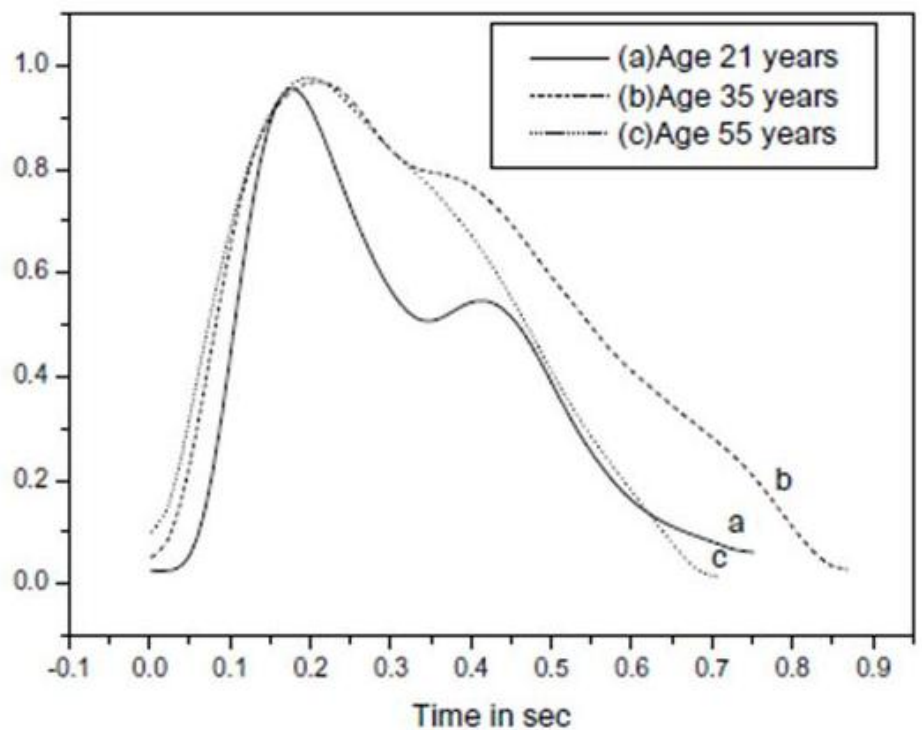

Figure 2.7: Blood Pressure Pulse Waveform at different Ages

(SOURCE: V. K. Jayasree, T. V. Sandhya, P. Radhakrishnan, "Non-invasive Studies on Age Related Parameters Using a Blood Volume Pulse Sensor”, Measurement Science Review, Vol. 8, Sec. 2, No. 2, pp. 82-86, 2008 [18]) 
Increase of PWV due to reduction of large arteries flexibility, can result in an early reflection of pressure wave with increase SBP and decrease DBP, and due to this phenomenon PWV remains as an independent indicator of cardiovascular risk event [37].

\subsection{Methods of Pulse Recording}

Most of blood pressure measurement devices are limited to measuring SBP and DBP only. Recently new techniques have been presented to record blood pressure pulse waveform as well. Most of these techniques record the waveform non-invasively; however, the pulse waveform can be extracted from invasive methods as well. Some of the non-invasive methods of extracting blood pressure pulse waveform are listed next:

\subsubsection{Photo-Plethysmography}

Photo-plethysmography (PPG) is a non-invasive technique to measure the changes in the blood flow in different part of individual's body. It can measure the pulsatile changes in blood volume in the micro-vascular bed. PPG pulse signals can easily be obtained from the tissue pads of the ear, fingers, and toes where there is a high degree of superficial vasculature [25] and provides a mean of determining properties of the vascular tree during the cardiac cycle and changes with aging and disease [18].

Using photo-plethysmography in BP measurement was first introduced on 2008. The device consisted of two major parts. First part was placed around the subject wrist to capture the signals from the arteries and the second part was placed at the subject's fingers. Pulse waveform could be constructed from the transit time of the circulatory pressure wave and the wave speed due to the distance of two devices [63]. 
PPG is a simple, inexpensive, compact, and portable technique to obtain blood pressure pulse waveform that make PPG being used in a wide range of commercially available medical devices. PPG is also used to measure oxygen saturation, blood pressure, and cardiac output. Although, the origin components of the PPG signals is not fully understood, even small movement of subjects can affect the blood pressure waveform. Most importantly, the blood pressure waveform provided by PPG method is not calibrated [18].

\subsubsection{Oscillometric Method}

Oscillometric blood pressure method is based on the oscillation of the blood flow (i.e. pulses). These oscillations are also visible from the sphygmomanometer during the auscultatory technique of blood pressure measurement and the idea of inventing Oscillometric method was from the oscillations of the sphygmomanometer [64]. These oscillations (pulses) can be extracted from the cuff deflation pressure waveform (Figure 2.8). In Figure 2.8, cuff deflation pressure waveform is shown which is extracted from Oscillometric recording. Oscillometric pulses can be observed at different pressure points of cuff deflation waveform. In Figure 2.8, Oscillometric pulses at the range of 117 to $125 \mathrm{mmHg}$ are shown. Oscillometric pulses change their shape and characteristics depending on the different level of arterial tree that the measurement was taken. Since the cuff deflation pressure waveform is a signal of pressure over time, pulses can be observed and studied at different pressure points. Most of the studies on blood pressure pulse morphology have used pulses extracted from PPG. However, in this thesis blood pressure pulse morphology was done based on Oscillometric pulses. Characteristics of the pulses were determined at different pressure points for different age groups. Also, Oscillometric blood pressure pulse morphology was used to propose a new method to estimate BP based on the characteristics of the oscillometric pulses [64] [65]. 

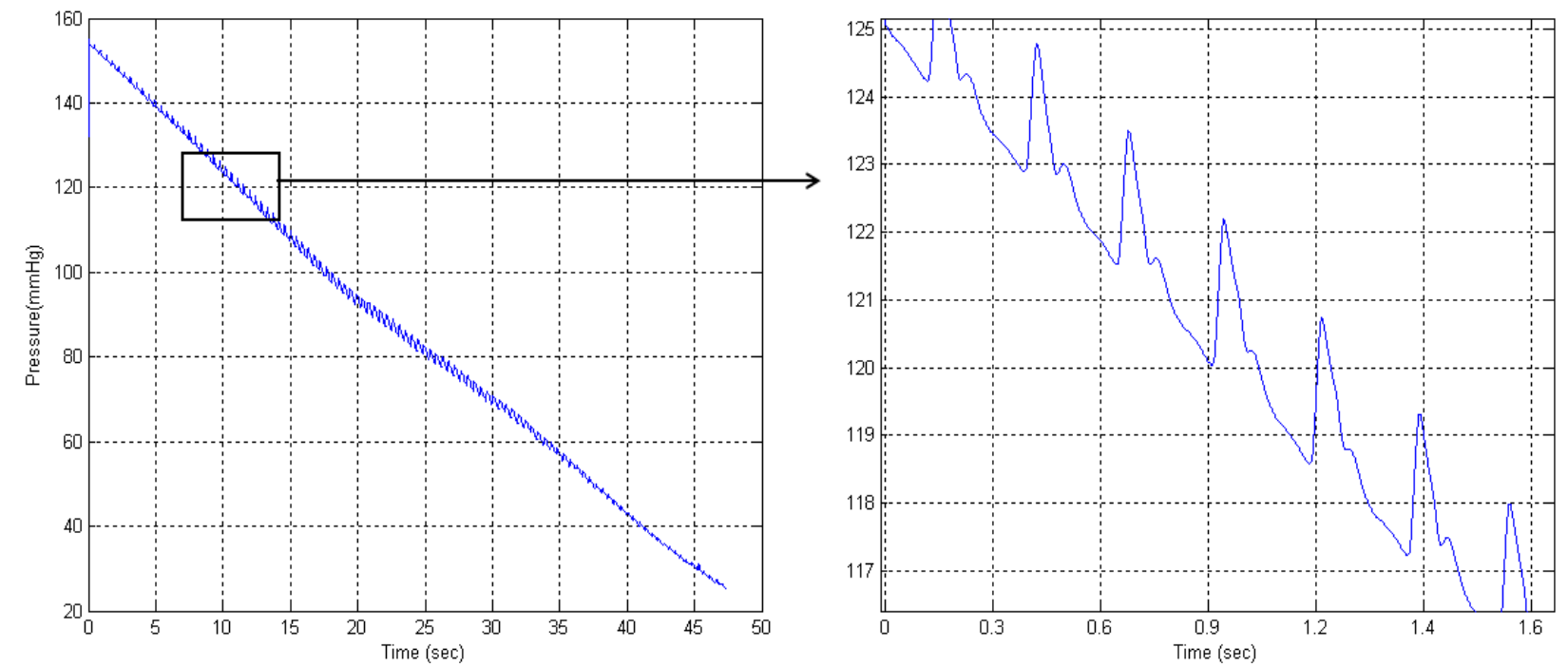

Figure 2.8: Oscillometric Blood Pressure Waveforms

\subsection{Pulse Morphology}

Pulse morphology is the subject of different investigation and it is mostly used to predict cardiovascular diseases. In previous studies, PPG was used, in which the pulse extracted at constant pressure were used. In pulse morphology, pulse characteristics were determined from the waveform and compared for different age groups. The pulse characteristics are either combination of pressure amplitudes in $\mathrm{mmHg}$ or time parameters of the pulse waveform. Some of the pulse characteristics are listed as follow:

\subsubsection{Stiffness Index (SI)}

The time difference between two peaks of blood pressure waveform $(\Delta \mathrm{T})$ can be used to infer the transit time taken for pressure to propagate along the aorta and large arteries to the major sites of the reflection in the lower part of body and back to the root of the arteries around the heart. This path is undefined and the length is unknown, but it can be assumed to be proportional to subject height (h). Therefor the stiffness of the artery can be formulated as [23]: 


$$
S I=\frac{h}{\Delta T}
$$

Due to the complexities of the formation of the blood pressure waveform, SI can be considered as a direct measure of the large arteries stiffness. It should be considered as an index characterizing features of the contour of pulse that are determined by PWV in the aorta and large arteries and by the stiffness of the arteries [23] [64].

\subsubsection{Augmentation Index (Al)}

As it was mentioned before, in older subjects increasing heart rate alerts pulse pressure amplification which is not associated with any change in aortic or other larger arteries stiffness and it can be determined by looking at Augmentation Index (AI) which is linearly and inversely related to heart rate [20] [35]. Augmentation Index can be calculated as follow:

$$
A I=\left[\frac{(F-P)}{F}\right] \times 100 \%
$$

$\mathrm{AI}$ is a pressure ratio and is defined as the difference of the systolic peak $(\mathrm{F})$ and second peak $(\mathrm{P})$ over systolic peak of pulse waveform (Figure 2.9), expressed as a percentage of the pulse pressure [20] [35] [42] [64] [65]. Diastolic peak is dependent of the existence of the dicrotic notch. Existence of the dicrotic notch helps to find the location of the second peak. In cases that P cannot be observed the first derivative of the pulse waveform can be used to find the location of the second peak as will be explained in Section 4.5. 


\subsubsection{Reflection Index (RI)}

Reflection index is an index to show the pressure reflected from the periphery side back to the arteries around the heart during diastole phase of the heart which can affect the second peak of the blood pressure pulse waveform. RI is influenced by stiffness of the arteries and has a linear relation with age. It is related to the vascular tone and is defined as the height of the second peak (P) relative to the height of the first peak $(\mathrm{F})$ and it is calculated as the following formula [30] [64] [65]:

$$
R I=\left[\frac{P}{F}\right] \times 100 \%
$$

\subsection{4 $\Delta \mathrm{T} / \mathrm{T}$ Ratio}

$\Delta \mathrm{T}$ defines time deference between two peaks of pulse waveform and represents the transit time taken for pressure to propagate along the aorta and other large arteries around the heart to the periphery region and back from periphery to the arteries around the heart and $\mathrm{T}$ is the duration of the pulse. This ratio will change by age due to the change in stiffness of arteries and disappearance of the dicrotic notch in older subjects. It is used to define a non-invasive measure of the large arteries stiffness [18] [20].

\subsubsection{Time Parameters of Blood Pressure Pulse Waveform}

By looking at a typical pressure pulse waveform the following parameters can be measured (Figure 2.9): Crest Time (CT): time to reach the maximum pressure in the left ventricle and arteries around the heart during systole phase and it is measured as the time from the beginning of the pulse until the first peak of the pulse. Dicrotic Wave Time (DWT): is the time to reach the maximum level of the pressure reflected by the periphery side back to the origin of the arteries 
around the heart during the diastole phase. DWT can be calculated by measuring the time from the origin of the pulse to the second peak of the pulse waveform (Figure 2.9). Total pulse duration $(\mathrm{T})$ which is the time of one cardiac cycle and is the duration of a single blood pressure pulse waveform. From the above presented parameters, the following secondary parameters can be derived. Relative Crest Time $(\mathrm{RCT})=\mathrm{CT} / \mathrm{DWT}$, Relative Dicrotic Wave Time $($ RDWT $)=$ DWT/T, and Time Delay Parameter (DT) $=$ DWT - CT [39] [64].

Change in the shape of the pulse contour due to the age, exercise, and cardiovascular diseases can affect the time parameter of the blood pressure pulse waveform.

\subsubsection{Systolic / Diastolic Slope}

The slope between systolic and diastolic peaks changes due to change of the location and amplitude of second peak of the pulse waveform, disappearance of dicrotic notch, and changes of time parameters of blood pressure pulse waveform with age and other cardiovascular diseases. It can be useful in pulse morphology and classification of different groups.

\subsubsection{Area under Systolic Curve}

Systolic area is the area under the pulse waveform between the origin of the waveform and the systolic peak and it would also change due to the change of flexibility of the large arteries in older subjects and people with cardiovascular diseases [18]. 


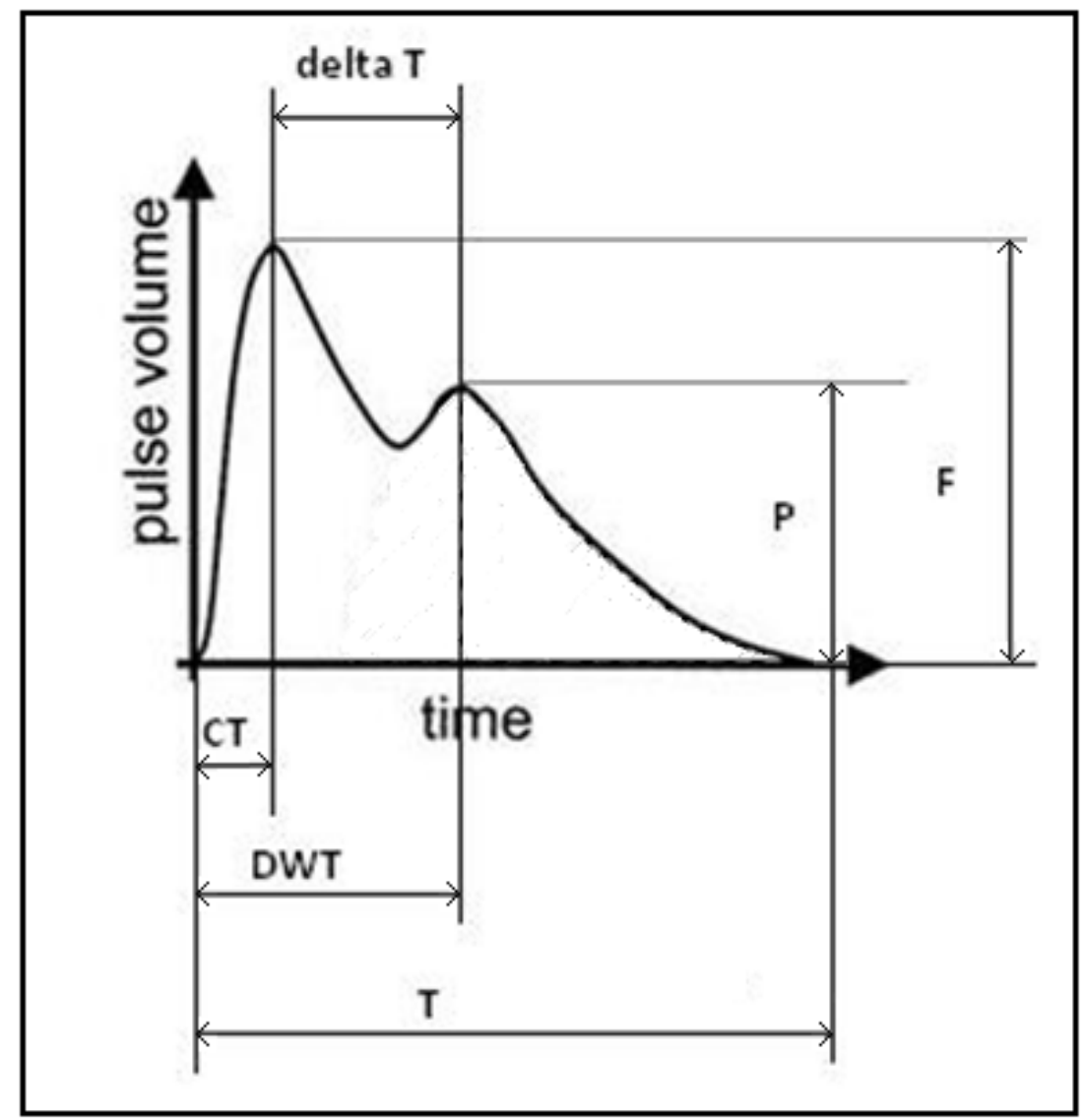

Figure 2.9: Blood Pressure Pulse Parameters

Table 2.1: Common usage of pulse parameters

\begin{tabular}{|l|l|}
\hline \multicolumn{1}{|c|}{ Parameter } & \multicolumn{1}{c|}{ Common Use } \\
\hline Stiffness Index (SI) & For artery stiffness [23] \\
\hline Augmentation Index (AI) & For artery stiffness [20] [35] [42] \\
\hline Reflection Index (RI) & For artery stiffness [30] \\
\hline$\Delta$ T/T Ratio & For artery stiffness and prediction of hypertension [18] [20] \\
\hline Time parameters of pulse waveform & Prediction of hypertension [39] \\
\hline Systolic/diastolic slope & For artery stiffness and prediction of hypertension \\
\hline Area under systolic curve & For artery stiffness and prediction of hypertension [18] \\
\hline
\end{tabular}




\subsection{Measurement Terminology}

Current work in Blood Pressure estimation is mostly in medicine and physiology field. The work done in this thesis is in engineering field. Therefore, short definitions of the most commonly used terms used in this thesis are provided to avoid any sort of confusion by possible readers in medicine field:

Signal: A detectable physical quantity or impulse by which information can be transmitted.

Measurement: Process or the result of determining physical information and quantity.

Recording: Process of capturing data or any physical information that can be digitally stored.

Waveform: Refers to the shape of the graph with varying quantity against time or distance.

Estimate: Approximate calculation of the values of any physical quantity.

Pulse: The arterial palpitation of heartbeat.

Error: The difference between the actual values that are estimated and the desired values.

Pulse Morphology: Description and analysis of a pulse based on its characteristics and parameters.

\subsection{Estimation Tools}

The end goal of this thesis work is to develop a new method of Blood Pressure estimation which is more robust than other existing methods of BP measurements based on the morphology of the pulse waveforms extracted from the cuff deflation pressure waveform and to check the developed method with the existing methods of BP estimation for improving the SBP and DBP 
estimation by Oscillometric devices. To achieve this goal, some algorithms have been used and evaluation of these algorithms needs understanding of some estimation tools.

In the comparison of Oscillometric pulse waveforms at different pressure points for every age group, Standard Deviation (STD) is used. STD is defined as the square root of the variance of the difference between the characteristics of the pulse waveforms. The characteristics of the pulse waveform can be either in pressure in unit of $\mathrm{mmHg}$ or time in unit of second.

In BP estimation, both Mean Absolute Error (MAE) and Standard Deviation (STD) are used. MAE is defined as the mean of the absolute error and STD is calculated as the square root of the variance of the error. Error can be calculated by the difference of the estimated blood pressure and the reference blood pressure in $\mathrm{mmHg}$. The reference is taken by OMRON blood pressure measurement device in this project. Both MAE and STD are common tools that used to evaluate the performance of different blood pressure algorithms [55] [61].

Statistical t-tests are used to show whether the mean of two different groups are different from each other. This method is usually used to compare the mean of two groups. It is a useful tool to check if any improvement achieved by any groups by checking the means of each group. It can statistically show that the two sets of results are different from each other and whether there is any improvement. Paired t-tests will be used for comparison of the proposed method of blood pressure estimation in this thesis with MAA. 


\section{Chapter 3: Methodology}

\subsection{Study Population}

Oscillometric waveforms were obtained from 18 healthy adults, aged from 24 to 68 years old (12 males, 6 females), with no history of cardiovascular diseases. They were also requested not to take any kind of medication during the period of the recording. All subjects provided informed consent form to the blood pressure measurement, in accordance with the guidelines of the institutional research ethic board (See Appendix for the Informed Consent Form).

\subsection{Prototypes Device}

All the recordings were taken using a blood pressure measurement prototype device which records Electrocardiogram (ECG) at the same time of estimating BP [47]. A cuff with conductive fabric at its inner side was wrapped around the subject's left arm. The conductive fabric served as the electrode for detecting ECG. Another conductive fabric was wrapped around the right wrist of the subject and served as the second electrode for detecting ECG. The cuff and conductive fabrics were connected to the device as shown in Figure 3.1.

The device contains four main components, namely, an analog ECG amplifier, an analog pressure transducer (Vernier Pressure Transducer BPS-BTA, Beaverton, OR, USA), a mini direct current (DC) air pump, and a screw that controlles manual pressure release valve. The core of the ECG amplifier consisted of an instrumentation amplifier (INA-129, Texas Instruments, Dallas, TX, USA) supported by circuitry for stabilizing the supply voltage and conditioning the signal. The analog voltage outputs from the ECG amplifier and the Vernier pressure transducer 
were fed to two simultaneously sampled analog input channels of a National Instruments ${ }^{\mathrm{TM}} \mathrm{C}$ Series 9239 analog input module (NI-9239) mounted on a Compact DAQ data acquisition board. These analog signals were conditioned using the NI 9239 module, buffered, and then sampled by a 24-bit delta-sigma analog-to-digital converter (ADC). The quantized signals were transmitted to a PC via a universal serial bus (USB) cable. A PC-based National Instruments ${ }^{\mathrm{TM}}$ LabVIEW development environment was used for acquiring and controlling the acquisition of ECG and cuff pressure signals. Both signals were acquired simultaneously at a sampling rate of $1000 \mathrm{~Hz}$. Software in Matlab® (The MathWorks Inc., Natick, MA, USA) was written for reading, processing, and analyzing the acquired pulse signals [47].

Form literature review, for healthy people, there should be a pulse within every R-peaks of ECG signals. Therefore, ECG signal was used to detect pulses to extract better envelope in MAA method which was used to compare with the presented method [47].

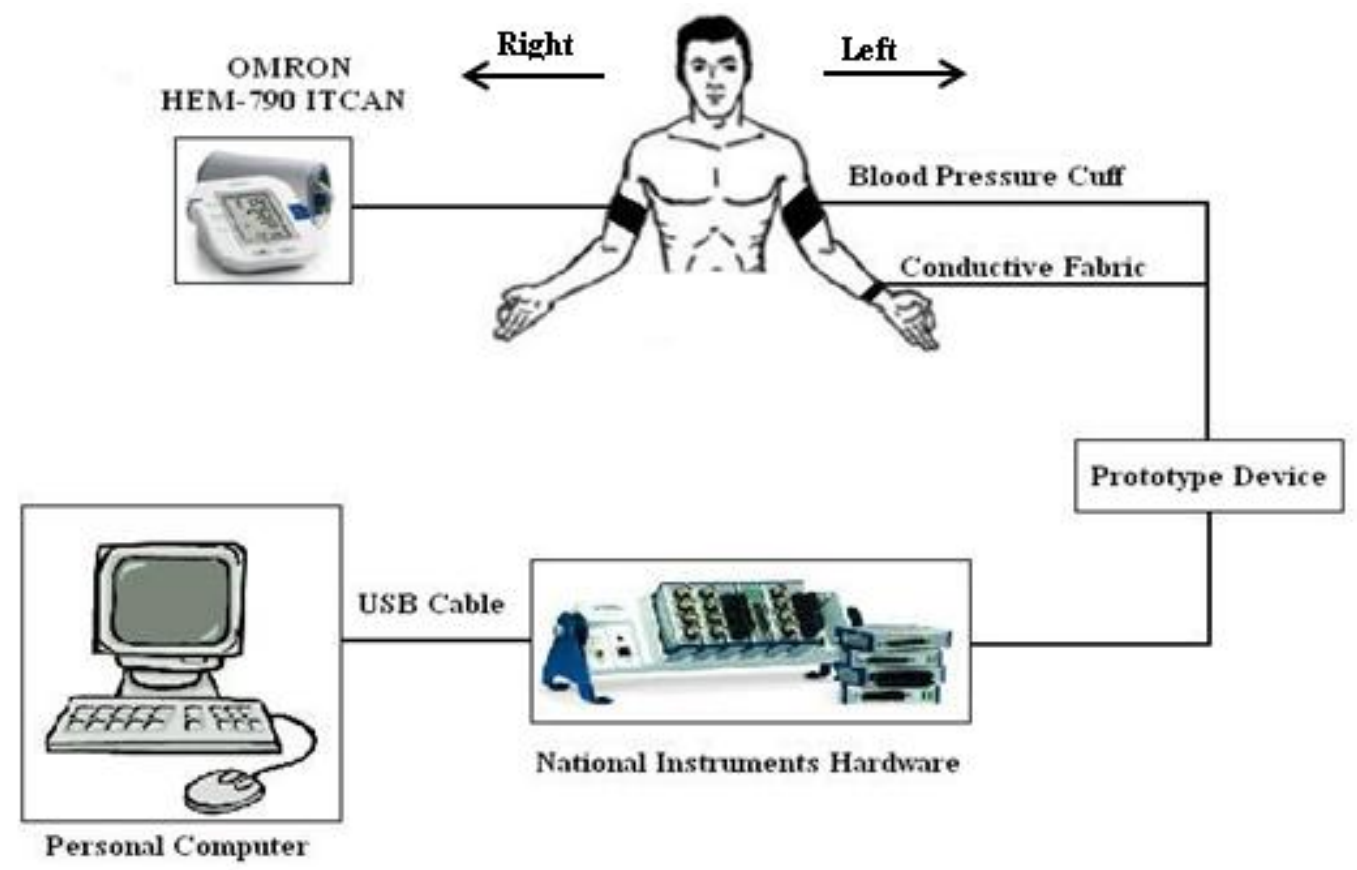

Figure 3.1: Block Diagram of BP Prototype Development 


\subsection{Reference Device and Algorithm}

OMRON HEWM-790 ITCAN is a commercial Oscillometric based blood pressure measurement device which is intended for home monitoring system. OMRON recordings were taken before our prototype device recording and its results were saved to use as a comparison of the presented method.

Maximum Amplitude Algorithm (MAA) method is a popular method of estimating blood pressure in electronic devices. SBP, MAP, and DBP were estimated using MAA method and used as our second reference to compare with the proposed method. An example of BP estimation using MAA method is shown in Figure 3.2. In this figure, horizontal lines represent the OMRON estimates of SBP and DBP. Maximum point shows the MAP (OPI) which was found using MAA. The point on the right side of the MAP corresponds to SBP and the point on the left side of the MAP corresponds to DBP.

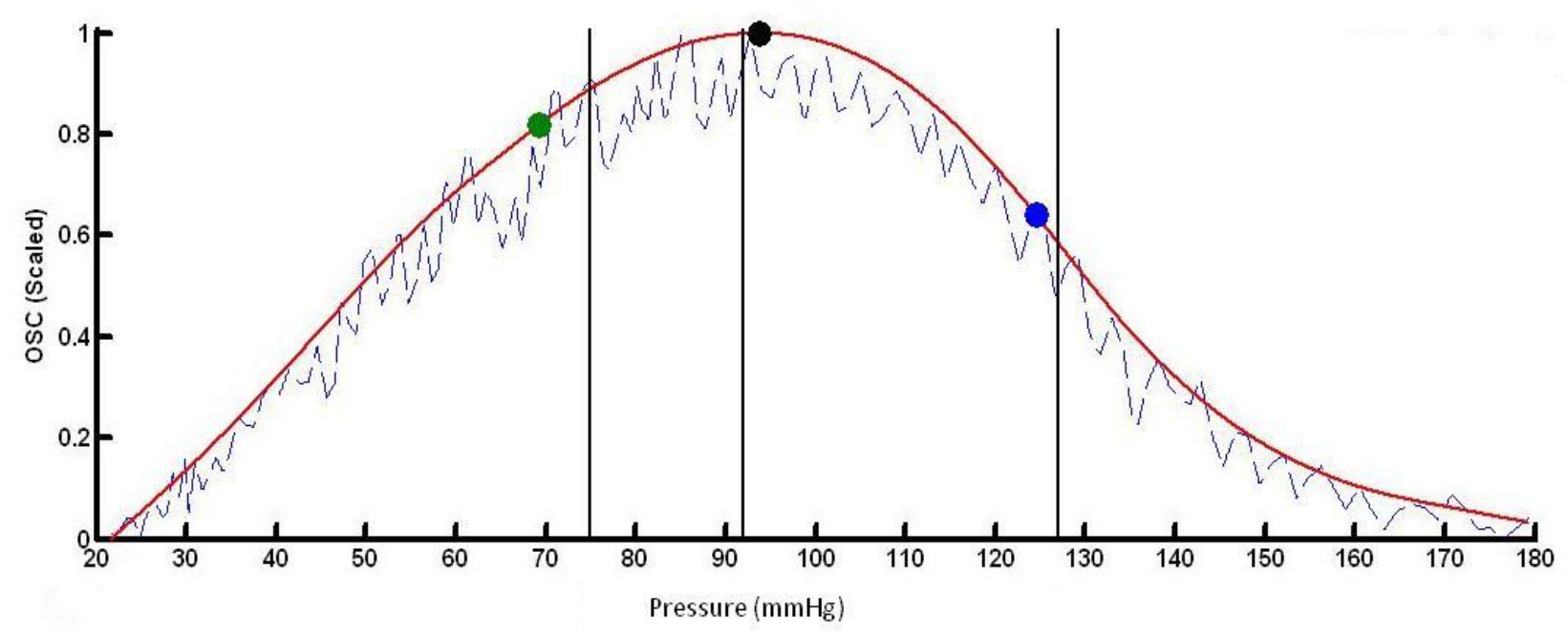

Figure 3.2: Reference results using OMRON recording and MAA method 


\subsection{Experimental Procedure}

The subjects were seated in a chair with their arm at the level of the heart during the recording and asked to be relaxed and breathe normally with possible minimum movement. Each recording took around 90 seconds with our prototype device and the entire session took around one hour. Subjects were asked to participate over 2-3 days with 5 trials per day.

The cuff of an OMRON monitor device (Model HEM-790ITCAN) was placed around subject's right arm and blood pressure was recorded before each trial. Following ANSI/AAMI SP10 standards, there was around 60 seconds delay between OMRON recording and actual recording with our prototype device [67]. Since the OMRON device only records the systolic and diastolic pressures, formula 2.1 was used to derive the Mean Arterial Pressure (MAP) from OMRON measurements.

After BP measurement with OMRON device, the cuff which was placed around subject's left arm was inflated above the expected SBP (around 160-180 $\mathrm{mmHg}$ ) and deflated slowly below the expected DBP (around $20 \mathrm{mmHg}$ ). Vernier Blood Pressure transducer provided the cuff deflation pressure waveform which represents the pressure of the cuff. Again, following SP10 standards, the resting period between consecutive trials was around 3 minutes. Figure 3.3 presents the 5 consecutive recordings by the prototype device.

MAA algorithm was applied on the recordings with the prototype device to estimate SBP, MAP and DBP. The estimated values were saved and with OMRON results were used as our references to be compared with the presented method. Table 3.1 shows the sequence of the experimental steps. 


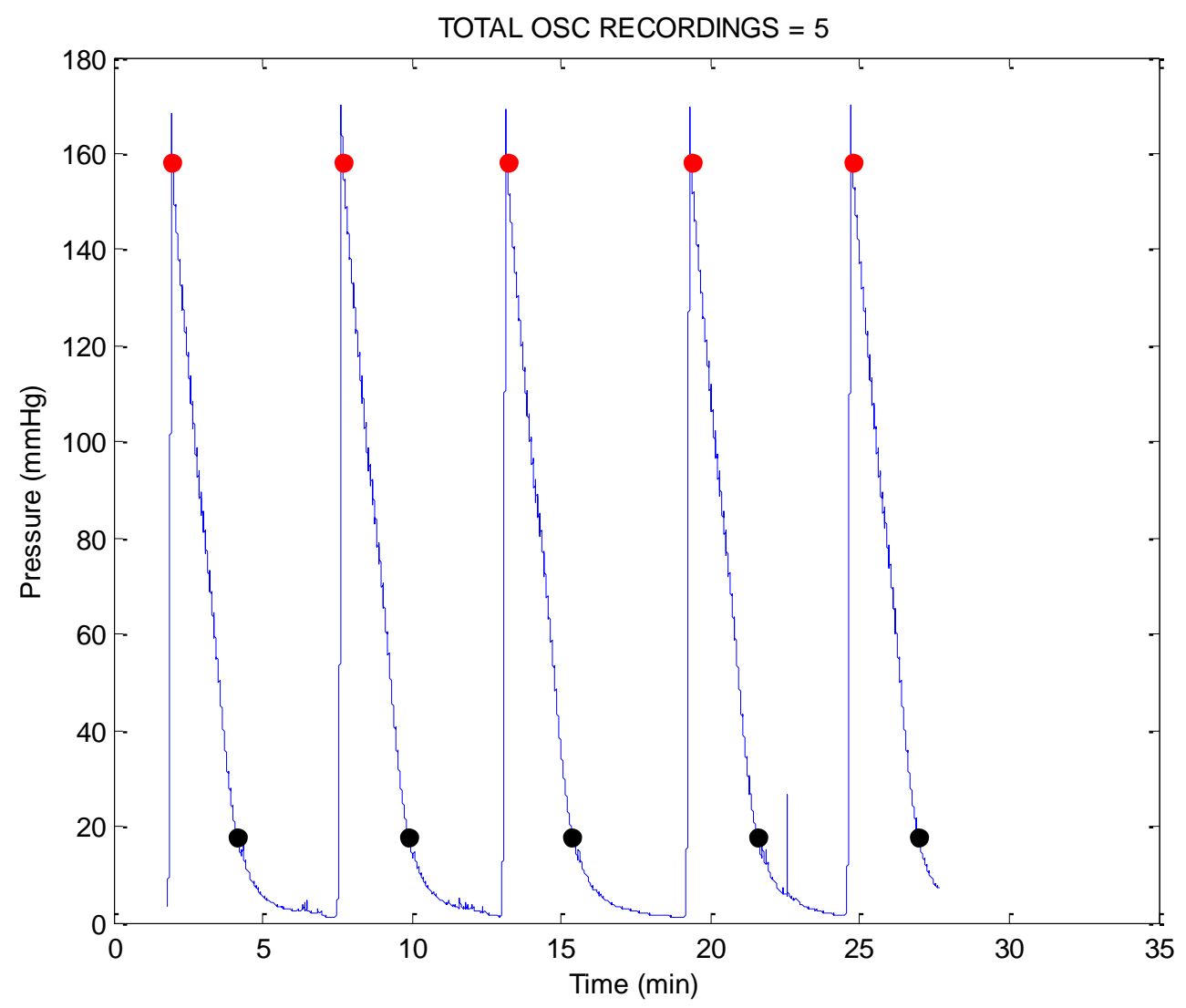

Figure 3.3: 5 Trials of Oscillometric Recording using the Prototype Device

Table 3.1: Experimental steps

\begin{tabular}{|l|l|}
\hline 1 & Place OMRON cuff around subject's right arm \\
\hline 2 & Place Prototype device's cuff around subject's left arm \\
\hline 3 & Make sure subject is relaxed and breathing normally \\
\hline 4 & Take OMRON measurement \\
\hline 5 & Wait around 1 minute \\
\hline 6 & Take measurements with prototype device \\
\hline 7 & Wait around 3 minutes \\
\hline 8 & Repeat steps $4,5,6$, and 7 five times \\
\hline
\end{tabular}




\subsection{Quantitative Measures}

After measuring SBP and DBP with OMRON device and measurements with prototype device, cuff deflation pressure waveforms were detected for all the recordings (Figure 2.2). All the pulses were detected from the cuff deflation pressure waveforms and the following quantitative measures that were explained in chapter 2 were obtained for every detected pulse and some of these parameters were utilized in the estimation of the systolic (SBP), diastolic (DBP), and mean arterial pressures (MAP).

1. Stiffness Index (SI)

2. Augmentation Index (AI)

3. Reflection Index (RI)

4. $\Delta \mathrm{T} / \mathrm{T}$ ratio

5. Time Parameters of blood pressure pulse waveform
a. Crest Time (CT)
b. Dicrotic Wave Time (DWT)
c. Total pulse duration $(\mathrm{T})$
d. Relative Crest Time (RCT)
e. Relative Dicrotic Wave Time (RDWT)
f. Time Delay parameter (DT)

6. Systolic/Diastolic Slope

7. Area under the systolic curve

8. Maximum slope of the pulse 


\section{Chapter 4: Experimental Results}

\subsection{Subjects Age Groups}

For the first part of the project, subjects were divided into three different age groups: young subjects ( $<30$ years old), middle age subjects (30-55 years old), and elderly subjects ( $>55$ years old). The presented parameters in Section 3.6 were obtained and studied for the pulses at systolic, MAP, and diastolic regions. Pulses at different pressure regions for randomly selected subjects from each age group are shown in Figure 4.1. In Figure 4.1, right column represents the systolic pulses for all age groups, middle column shows examples of MAP pulses for all age groups, and left column represents examples of diastolic pulses for all the age groups.

\subsection{Oscillometric Pulse Morphology}

As mentioned earlier in Section 1.4, three main parameters are needed to be extracted from each pulse which help to calculate the characteristics of the pulse. These parameters help to study the presented quantitative measures in Section 3.6, namely:

1. Existence of dicrotic notch

2. Amplitude of the peaks or pressure parameters in $\mathrm{mmHg}$

3. Time parameters of each peak

- Peak locations and time difference between peaks locations

Existence of the dicrotic notch is important to find the location and amplitude of the second peak of the pulse waveform. In some cases the dicrotic notch becomes less or not visible and finding 
the second peak of the pulse is an issue. In these cases, first derivative of the pulses is used to find the location of the second peak, explained in Section 4.4.
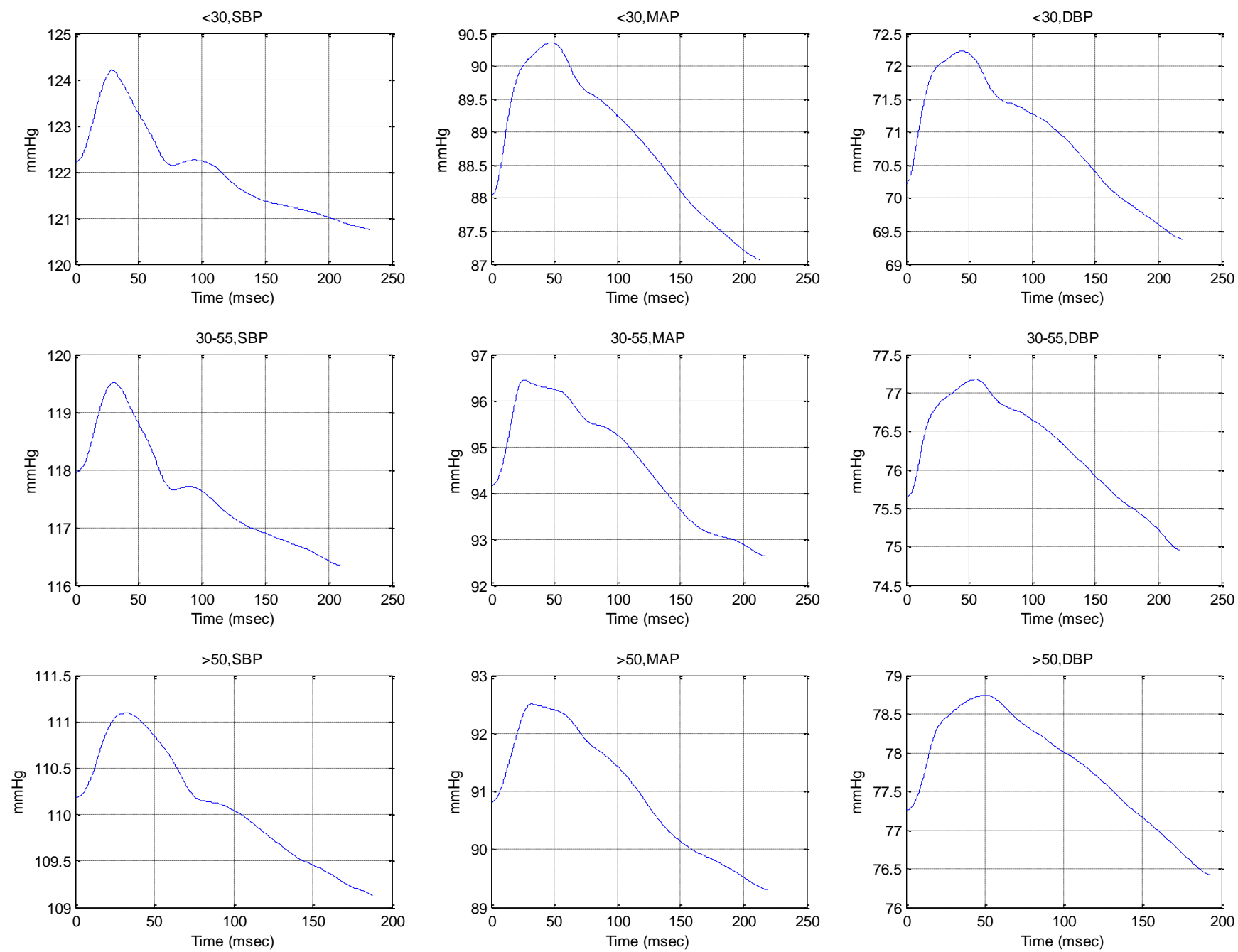

Figure 4.1: Sample Pulses at different Pressure Points for Subjects from different Age Groups

All the parameters presented in Section 3.6 obtained from the pulses at systolic, MAP, and diastolic regions for all 18 subjects. Pulses showed to change their shape and characteristics at different pressure points. Both time and pressure parameters of the pulses varied at different locations of the cuff deflation pressure waveform. 
Parameters of pulse waveform at different age groups and different pressure regions are presented in Tables $4.1-4.10$. Since the pulses have the cuff effect and the beginning and the end of the waveforms are not in the same level, the end point of the pulse waveforms were assumed to be the base of the pulses to estimate the amplitudes of the peaks.

Table 4.1: Reflection Index

\begin{tabular}{|c|c|c|c|}
\hline $\mathrm{RI} \pm \mathrm{STD}$ & SBP & MAP & DBP \\
\hline$<30$ & $0.43 \pm 0.03$ & $0.75 \pm 0.07$ & $0.70 \pm 0.09$ \\
\hline $30-55$ & $0.35 \pm 0.05$ & $0.63 \pm 0.11$ & $0.69 \pm 0.03$ \\
\hline$>55$ & $0.22 \pm 0.06$ & $0.58 \pm 0.06$ & $0.54 \pm 0.09$ \\
\hline
\end{tabular}

Table 4.2: Augmentation Index

\begin{tabular}{|c|c|c|c|}
\hline $\mathrm{AI} \pm \mathrm{STD}$ & $\mathrm{SBP}$ & MAP & $\mathrm{DBP}$ \\
\hline$<30$ & $57.53 \pm 3.24$ & $26.79 \pm 1.47$ & $24.76 \pm 3.51$ \\
\hline $30-55$ & $52.36 \pm 2.86$ & $24.28 \pm 2.01$ & $19.81 \pm 3.04$ \\
\hline$>55$ & $50.49 \pm 3.61$ & $21.81 \pm 1.96$ & $17.60 \pm 2.92$ \\
\hline
\end{tabular}

Table 4.3: Stiffness Index

\begin{tabular}{|c|c|c|c|}
\hline SI \pm STD & SBP & MAP & DBP \\
\hline$<30$ & $0.68 \pm 0.04$ & $1.21 \pm 0.04$ & $1 \pm 0.04$ \\
\hline $30-55$ & $0.78 \pm 0.06$ & $0.73 \pm 0.03$ & $1.27 \pm 0.03$ \\
\hline$>55$ & $0.89 \pm 0.06$ & $0.73 \pm 0.02$ & $1.32 \pm 0.01$ \\
\hline
\end{tabular}

Table 4.4: $\Delta \mathrm{T} / \mathrm{T}$ ratio

\begin{tabular}{|c|c|c|c|}
\hline$\Delta \mathrm{T} / \mathrm{T} \pm \mathrm{STD}$ & $\mathrm{SBP}$ & $\mathrm{MAP}$ & $\mathrm{DBP}$ \\
\hline$<30$ & $0.27 \pm 0.01$ & $0.17 \pm 0.06$ & $0.10 \pm 0.01$ \\
\hline $30-55$ & $0.27 \pm 0.03$ & $0.19 \pm 0.05$ & $0.16 \pm 0.01$ \\
\hline$>55$ & $0.25 \pm 0.03$ & $0.29 \pm 0.06$ & $0.17 \pm 0.01$ \\
\hline
\end{tabular}


Table 4.5: Crest Time

\begin{tabular}{|c|c|c|c|}
\hline $\mathrm{CT} \pm \mathrm{STD}$ & SBP & MAP & DBP \\
\hline$<30$ & $129 \pm 5.36$ & $147 \pm 5.03$ & $173 \pm 6.12$ \\
\hline $30-55$ & $132 \pm 7.94$ & $161 \pm 3.71$ & $225 \pm 8.63$ \\
\hline$>55$ & $153 \pm 4.06$ & $189 \pm 8.27$ & $237 \pm 9.01$ \\
\hline
\end{tabular}

Table 4.6: Dicrotic Wave Time

\begin{tabular}{|c|c|c|c|}
\hline DWT \pm STD & SBP & MAP & DBP \\
\hline$<30$ & $388 \pm 15.65$ & $350 \pm 7.40$ & $350 \pm 16.03$ \\
\hline $30-55$ & $370 \pm 12.41$ & $370 \pm 15.81$ & $360 \pm 7.64$ \\
\hline$>55$ & $350 \pm 16.36$ & $350 \pm 8.94$ & $340 \pm 13.52$ \\
\hline
\end{tabular}

Table 4.7: Relative Crest Time

\begin{tabular}{|c|c|c|c|}
\hline RCT \pm STD & SBP & MAP & DBP \\
\hline$<30$ & $0.33 \pm 0.01$ & $0.41 \pm 0.04$ & $0.49 \pm 0.05$ \\
\hline $30-55$ & $0.36 \pm 0.03$ & $0.49 \pm 0.06$ & $0.63 \pm 0.06$ \\
\hline$>55$ & $0.44 \pm 0.04$ & $0.51 \pm 0.08$ & $0.65 \pm 0.07$ \\
\hline
\end{tabular}

Table 4.8: Relative Dicrotic Wave Time

\begin{tabular}{|c|c|c|c|}
\hline RDWT \pm STD & SBP & MAP & DBP \\
\hline$<30$ & $0.41 \pm 0.07$ & $0.40 \pm 0.05$ & $0.40 \pm 0.09$ \\
\hline $30-55$ & $0.42 \pm 0.05$ & $0.42 \pm 0.06$ & $0.41 \pm 0.06$ \\
\hline$>55$ & $0.44 \pm 0.06$ & $0.45 \pm 0.05$ & $0.44 \pm 0.09$ \\
\hline
\end{tabular}

Table 4.9: Time Delay Parameter

\begin{tabular}{|c|c|c|c|}
\hline TDP \pm STD & SBP & MAP & DBP \\
\hline$<30$ & $259 \pm 9.34$ & $146 \pm 6.94$ & $177 \pm 10.64$ \\
\hline $30-55$ & $238 \pm 11.06$ & $254 \pm 7.73$ & $135 \pm 8.51$ \\
\hline$>55$ & $197 \pm 8.36$ & $221 \pm 11.45$ & $124 \pm 7.09$ \\
\hline
\end{tabular}


Table 4.10: Area under Systolic Curve

\begin{tabular}{|c|c|c|c|}
\hline $\begin{array}{c}\text { Area under } \pm \text { STD } \\
\text { systolic }\end{array}$ & SBP & MAP & DBP \\
\hline$<30$ & $105 \pm 12.63$ & $141 \pm 7.35$ & $160 \pm 14.30$ \\
\hline $30-55$ & $80 \pm 9.45$ & $116 \pm 9.86$ & $143 \pm 12.06$ \\
\hline$>55$ & $75 \pm 9.36$ & $104 \pm 12.73$ & $136 \pm 8.64$ \\
\hline
\end{tabular}

Reflection Index, Augmentation Index, $\Delta \mathrm{T} / \mathrm{T}$ ratio, Dicrotic Wave Time, Time Delay Parameter, and area under systolic curve showed to decrease by age in all pressure points while, Stiffness Index, Crest Time, Relative Crest Time, and Relative Dicrotic Wave Time showed to mostly increase by age at all the pressure points. AI, $\Delta \mathrm{T} / \mathrm{T}$ ratio, DWT, and RDWT decreased by going from systolic pressure to diastolic pressure in cuff deflation pressure waveform while CT, RCT, and area under systolic curve increased by going from systolic pressure to diastolic pressure in cuff deflation pressure waveform. Reflection index had its maximum value at MAP and Stiffness Index had its maximum value at diastolic pressure.

\subsection{Blood Pressure Estimation using Pulse Morphology}

Oscillometric pulse morphology can be used to estimate SBP, MAP, and DBP. Pulses showed to change their shape and characteristics at different pressure points across the cuff deflation pressure waveform. Four parameters, namely: Augmentation Index (AI), Reflection Index (RI), Stiffness Index (SI), and $\Delta \mathrm{T} / \mathrm{T}$ ratio which were presented earlier, were obtained for all the pulses on cuff deflation pressure waveform and used to estimated BP.

As explained in chapter two, Augmentation Index and Reflection Index are pressure parameters of pulse waveform and are estimated from amplitudes of both peaks of the pulse. Stiffness Index 
is an indicator of the stiffness of the arteries and is estimated by dividing the height of the subjects by the height. $\Delta \mathrm{T} / \mathrm{T}$ ratio is a time ratio when $\Delta \mathrm{T}$ is the time difference of the peaks and $\mathrm{T}$ is total duration of the pulse waveform.

The plots of four quantitative measures were sketched and two different types of graphs were obtained. The plots for Reflection Index and Stiffness Index showed to be similar (Figure 4.2, Figure 4.3) while Augmentation Index and $\Delta \mathrm{T} / \mathrm{T}$ ratio plots were similar to each other (Figure 4.4, Figure 4.5).

These two types of graphs have opposite characteristics, but same technique was used to estimated SBP, MAP, and DBP. The x-axis of all the graphs represents the pulse numbers from cuff deflation pressure waveform in order. Therefore, pulses representing SBP, MAP, and DBP were detected and mapped to the cuff deflation waveform to obtain the corresponding pressures in $\mathrm{mmHg}$.

Similar to other techniques of estimating BP in Oscillometric method, MAP (OPI) divided the graph into systolic and diastolic regions. Left side of MAP corresponds to systolic and right side of MAP corresponds to diastolic pressures.

In type one (plots of Reflection Index and Stiffness Index), the maximum point corresponds to the pulse which represents MAP. The left side of MAP (systolic region) is convex and minimum point of systolic region corresponds to systolic pulse. Opposite to the systolic region, the maximum point of diastolic region represents diastolic pulse. All the pulses are mapped to the cuff deflation pressure waveform to obtain their corresponding pressures (Figure 4.2 and Figure 4.3). 
Type two (plots of Augmentation Index and $\Delta \mathrm{T} / \mathrm{T}$ ratio) has opposite characteristics of type one. The minimum point represents MAP pulse, systolic region is concave and maximum point of systolic region is used to obtain SBP. The diastolic region is convex and the minimum point is used to estimate DBP (Figure 4.4 and Figure 4.5).

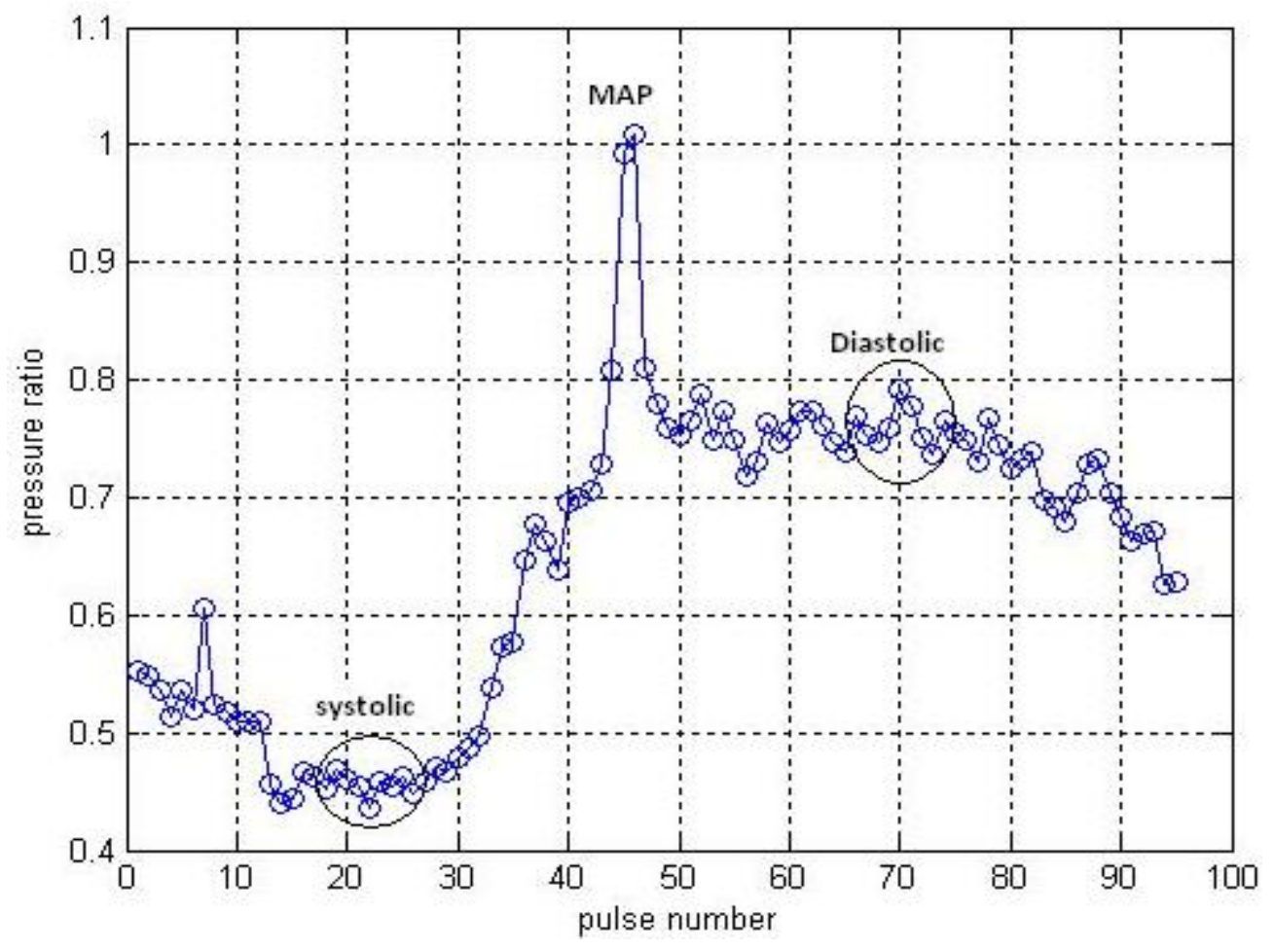

Figure 4.2: Plot of Reflection Index 


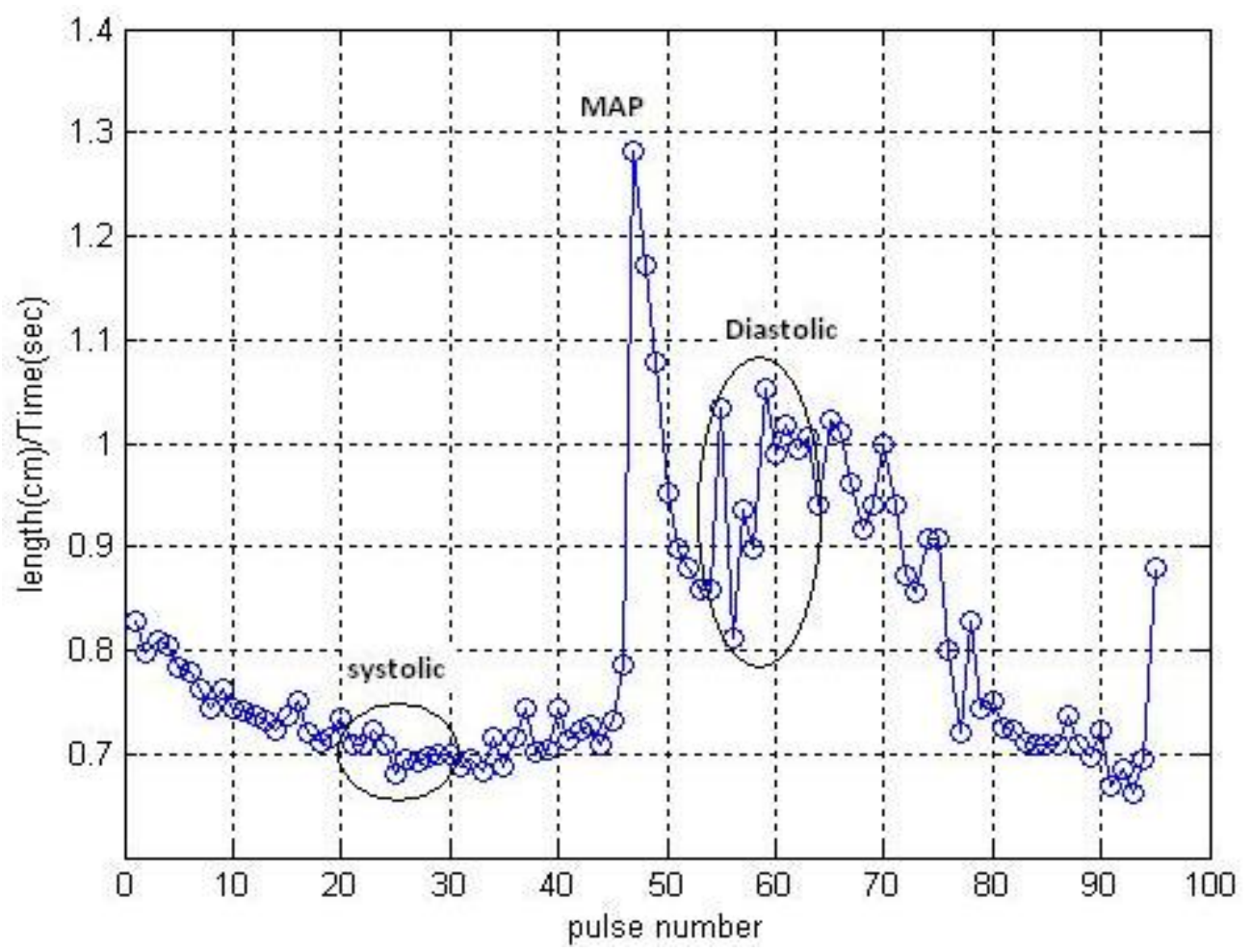

Figure 4.3: Plot of Stiffness Index

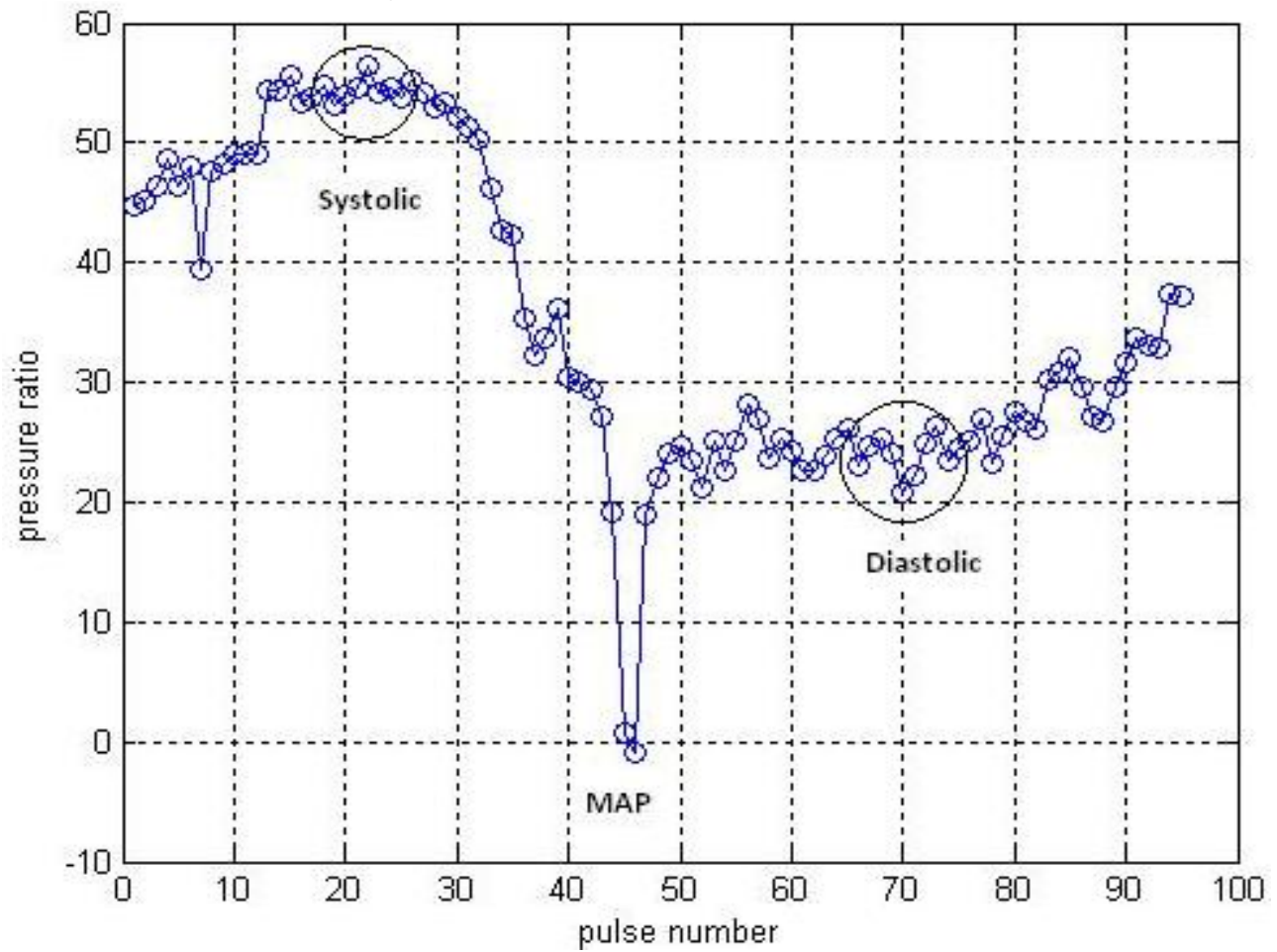

Figure 4.4: Plot of Augmentation Index 


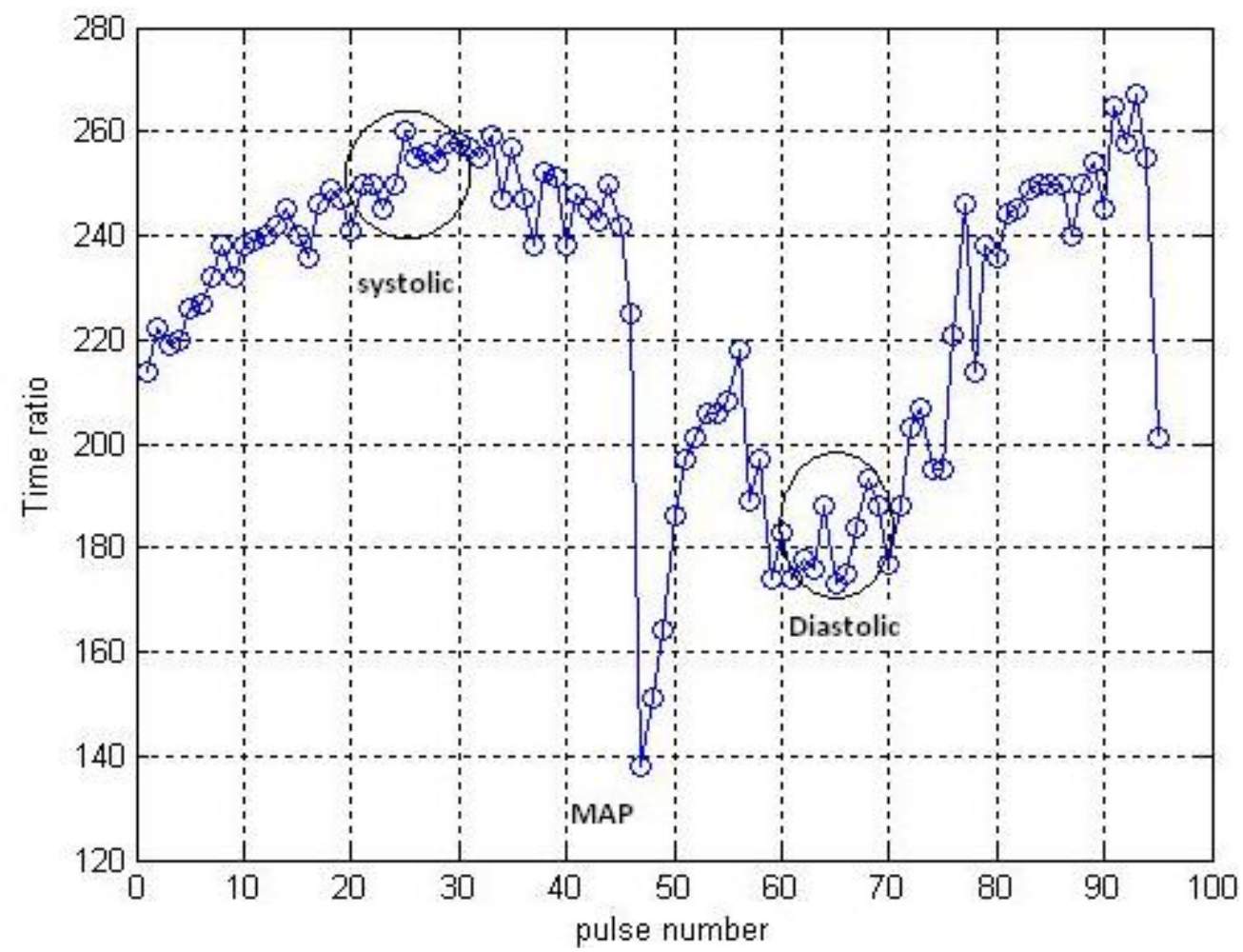

Figure 4.5: Plot of $\Delta \mathrm{T} / \mathrm{T}$ ratio

Since every pulse has a different pressure range (Figure 4.6), the average of the maximum and minimum pressures in the selected pulses is taken as pulse pressure.

Subjects may have small movements of their limbs during the recordings which can cause a source of error, to avoid unpredictable error in estimation of SBP and DBP using the proposed method, nine pulses at systolic and diastolic regions were picked at minimum and maximum regions and mean and median of the selected pulses were used to obtain SBP and DBP.

Estimated systolic, MAP, and diastolic blood pressures from this method were compared to the results of OMRON HEM-790ITCAN blood pressure measurement device and Maximum Amplitude Algorithm (MAA). The Mean Absolute Error (MAE) for comparison of the presented method and MAA with OMRON results at SBP, MAP, and DBP are presented at Tables 4.11 4.13. MAE of the proposed method at SBP and MAP was less than MAA in comparison with 


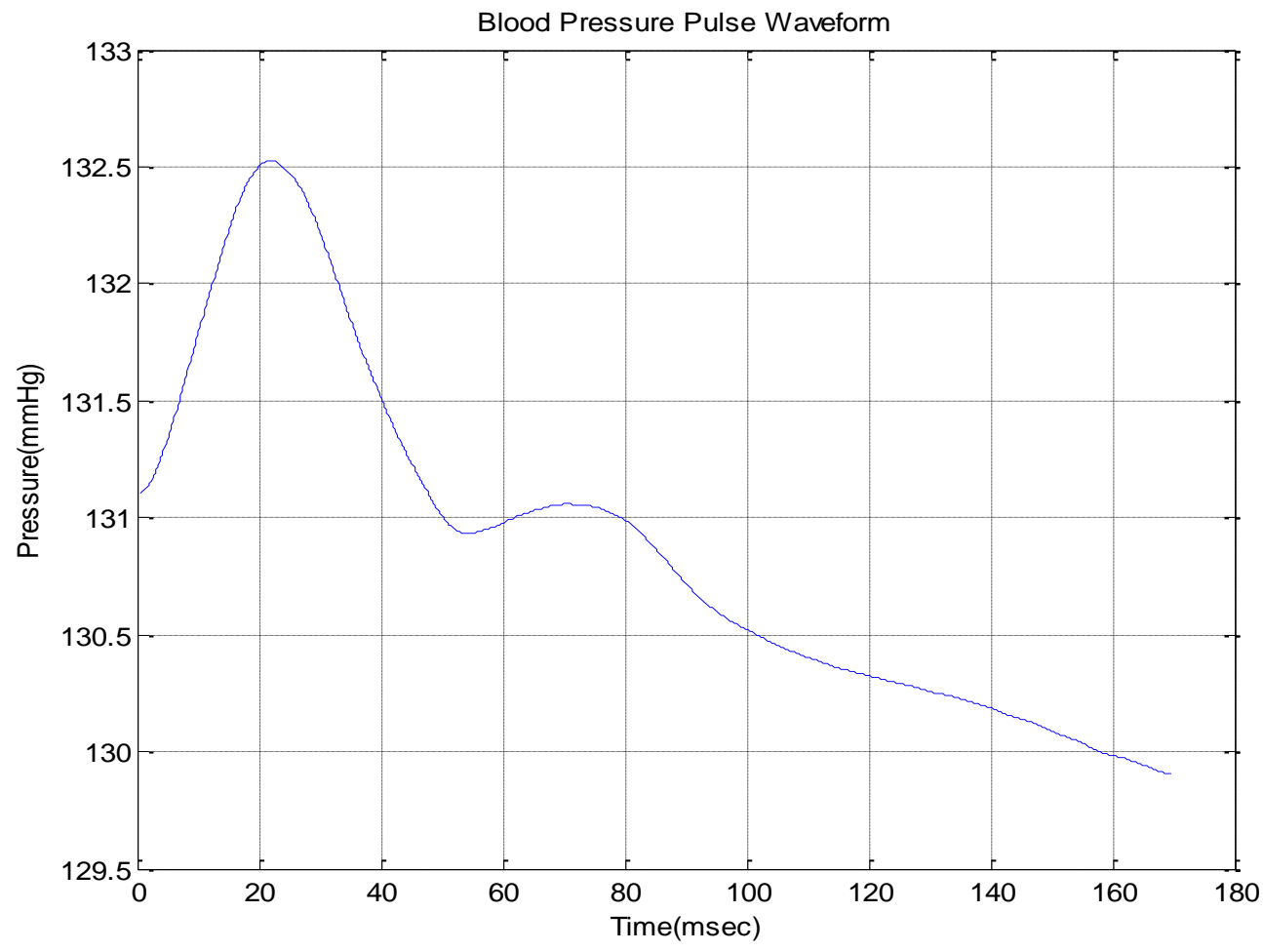

Figure 4.6: Different Pressure Range in a Pulse

OMRON results. MAE of MAA at DBP was less than the proposed method in comparison with OMRON results.

Table 4.11: Mean Absolute Error (mmHg) with OMRON results at SBP

\begin{tabular}{|c|c|c|c|c|c|}
\hline SBP & Augmentation Index & Reflection Index & Stiffness Index & $\Delta \mathrm{T} / \mathrm{T}$ & MAA \\
\hline OMRON & 4.7 & 4.6 & 3.3 & 3.2 & 4.9 \\
\hline
\end{tabular}

Table 4.12: Mean Absolute Error (mmHg) with OMRON results at MAP

\begin{tabular}{|c|c|c|c|c|c|}
\hline MAP & Augmentation Index & Reflection Index & Stiffness Index & $\Delta \mathrm{T} / \mathrm{T}$ & MAA \\
\hline OMRON & 2.1 & 2.1 & 2 & 2.2 & 3.7 \\
\hline
\end{tabular}


Table 4.13: Mean Absolute Error (mmHg) with OMRON results at DBP

\begin{tabular}{|c|c|c|c|c|c|}
\hline SBP & Augmentation Index & Reflection Index & Stiffness Index & $\Delta \mathrm{T} / \mathrm{T}$ & MAA \\
\hline OMRON & 5.3 & 5.1 & 4.9 & 4.9 & 4.7 \\
& & & & & \\
\hline
\end{tabular}

Bland Altman plots are also constructed for all the results from the presented method and OMRON recording shown in Figure 4.7 to Figure 4.10. These plots are constructed for each of the four quantitative measures. SBP, MAP, and DBP estimated from the presented algorithm are compared to the OMRON results and plotted in four figures. Lines which represent the mean difference between the reading and limits are shown in the plots. The limits of agreement are computed as the mean difference \pm 1.96 the standard deviation $(\mathrm{SD})$. The horizontal lines represent the mean difference and limits of agreements in each Bland Altman plot. Therefore, concentration of Bland Altman results in the middle of limit of agreements means more similarity between the estimated BP with proposed method and OMRON results.

SBP, MAP, and DBP of all the subjects' recordings with presented method, MAA, and OMRON are shown in Table 4.14 to Table 4.16. Comparison of all trials of all 18 subjects with MAA and OMRON are presented in Appendix A.

Table 4.14: Comparison of the presented method with MAA and OMRON results at SBP

\begin{tabular}{|c|c|c|c|c|c|c|}
\hline $\mathrm{SBP} \pm \mathrm{SD}$ & Augmentation Index & Reflection Index & Stiffness Index & $\Delta \mathrm{T} / \mathrm{T}$ & MAA & OMRON \\
\hline $\mathrm{mmHg}$ & $113.8 \pm 7.9$ & $113.77 \pm 7.9$ & $112.83 \pm 8.4$ & $113.61 \pm 8.3$ & $114.06 \pm 6.7$ & $114.13 \pm 9.2$ \\
\hline
\end{tabular}


Table 4.15: Comparison of the presented method with MAA and OMRON results at MAP

\begin{tabular}{|c|c|c|c|c|c|c|}
\hline $\mathrm{MAP} \pm \mathrm{SD}$ & Augmentation Index & Reflection Index & Stiffness Index & $\Delta \mathrm{T} / \mathrm{T}$ & MAA & OMRON \\
\hline $\mathrm{mmHg}$ & $86.8 \pm 7.2$ & $87.12 \pm 7.2$ & $87.13 \pm 6.57$ & $87.11 \pm 6.56$ & $87.64 \pm 4.73$ & $87 \pm 5.42$ \\
\hline
\end{tabular}

Table 4.16: Comparison of the presented method with MAA and OMRON results at DBP

\begin{tabular}{|c|c|c|c|c|c|c|}
\hline $\mathrm{DBP} \pm \mathrm{SD}$ & Augmentation Index & Reflection Index & Stiffness Index & $\Delta \mathrm{T} / \mathrm{T}$ & MAA & OMRON \\
\hline $\mathrm{mmHg}$ & $74.17 \pm 5.77$ & $74.08 \pm 5.7$ & $75.04 \pm 5.56$ & $73.94 \pm 5.4$ & $72.82 \pm 8.7$ & $72.52 \pm 4.8$ \\
\hline
\end{tabular}



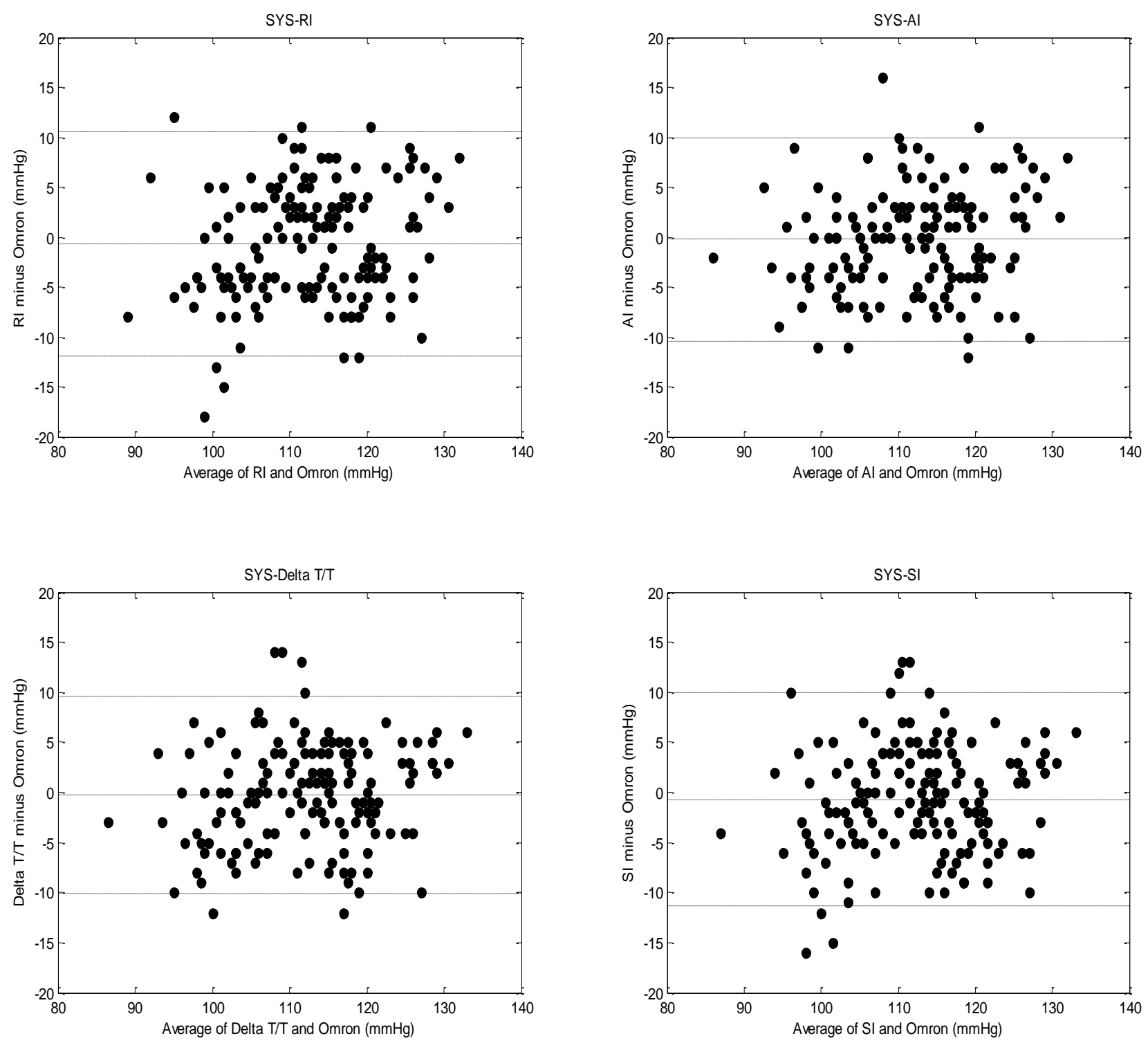

Figure 4.7: Bland Altman plot comparing the Presented Method with OMRON results in SBP 

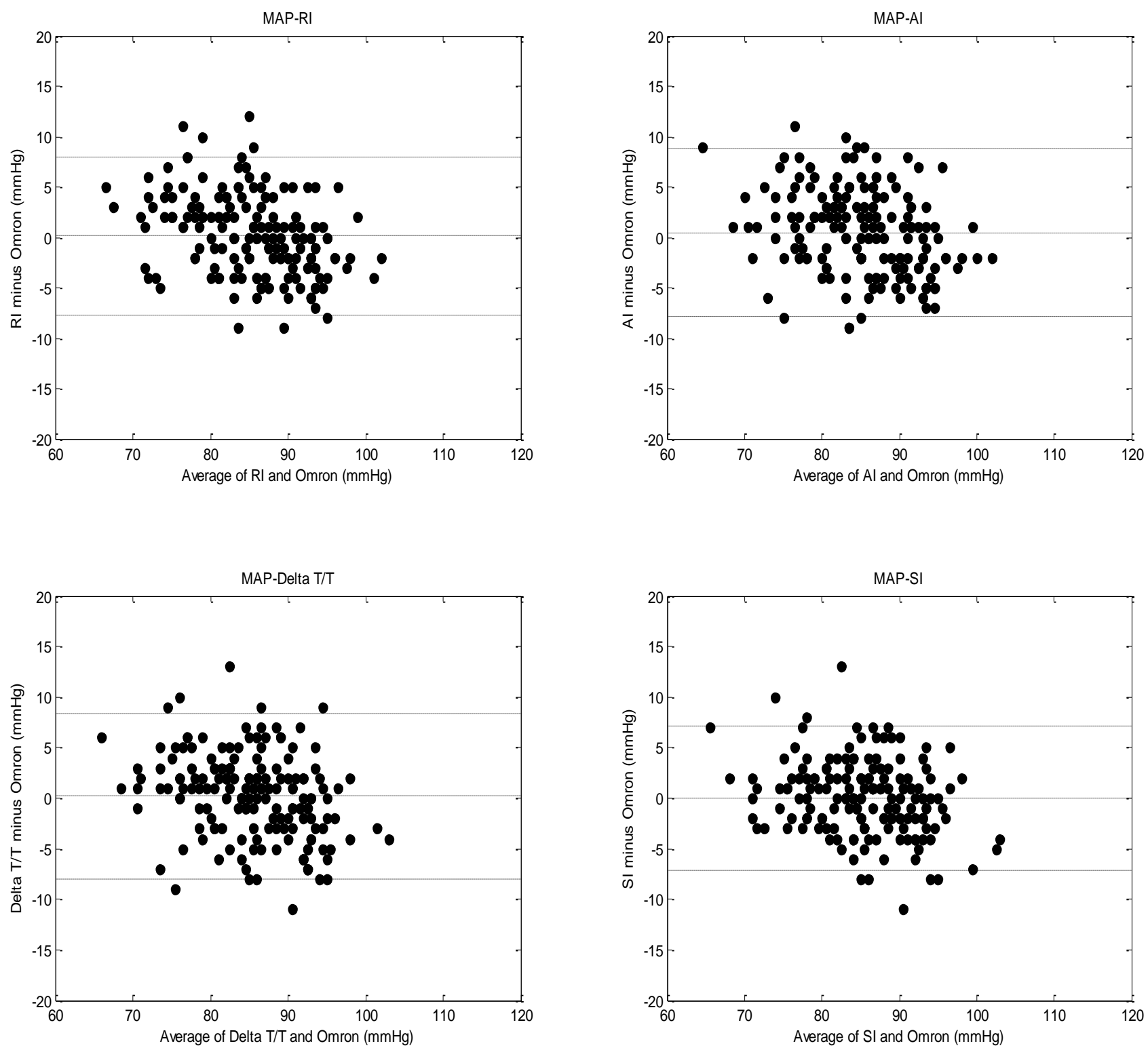

Figure 4.8: Bland Altman plot comparing the Presented Method with OMRON results in MAP 

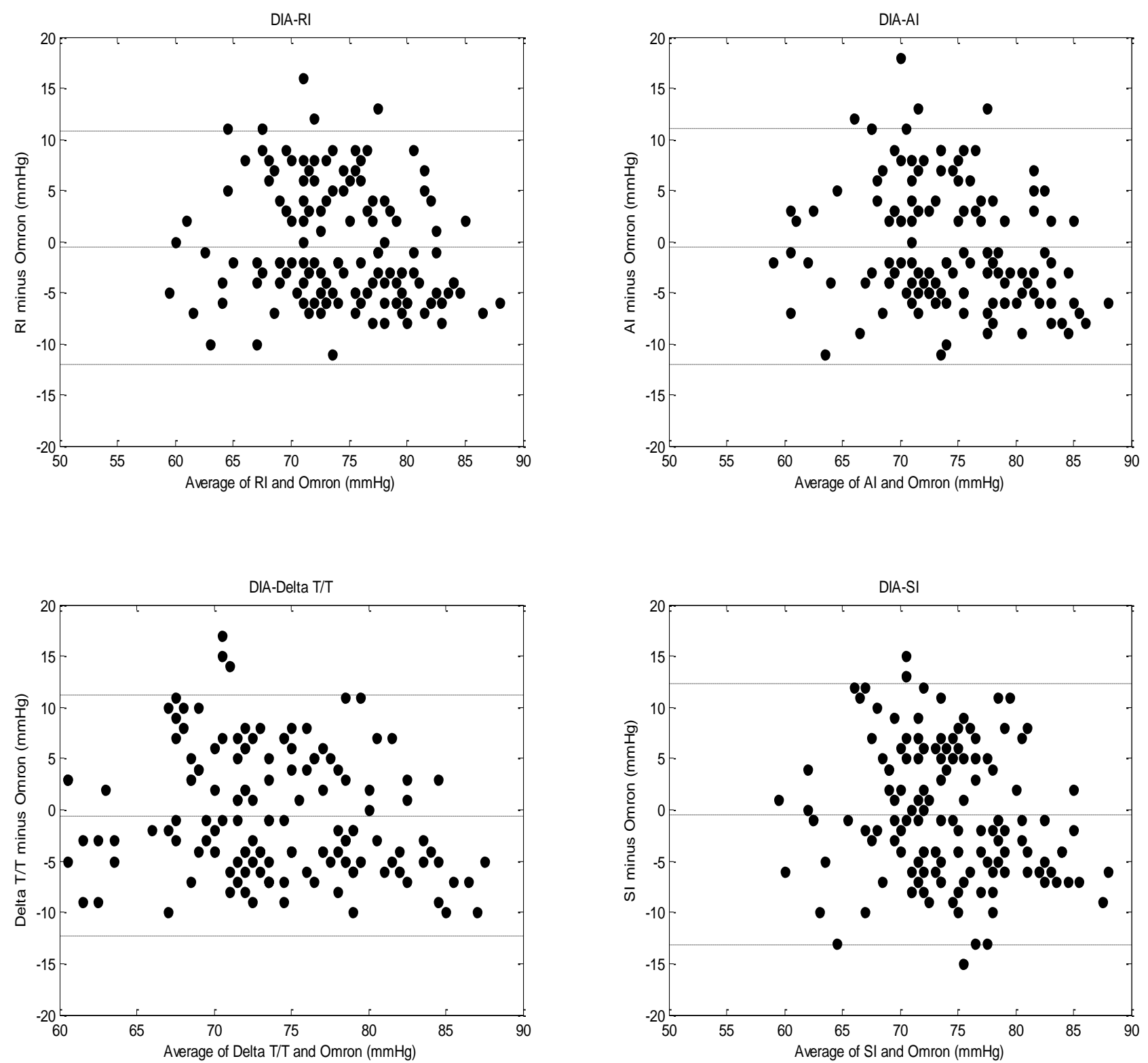

Figure 4.9: Bland Altman plot comparing the Presented Method with OMRON results in DBP 


\subsection{SBP and DBP Estimation using Curve Fitting Technique}

Systolic and diastolic blood pressures were obtained from fitted curves as well. The plots of 4 parameters of all pulse waveforms were smoothed using curve fitting technique. Smooth Spline method of curve fitting was used for smoothing the plots of parameters. Since the pulses representing systolic and diastolic regions were detected from the minimum and maximum regions of the convex and concave graphs respectively, the first derivative of the smoothed curves was used to detect the location of the maxima and minima of the convex and concave graphs. The maximum point of concave graphs was obtained when the graph of first derivative was going from positive region to negative region. Also, the minimum point of convex graphs was obtained when the graph of first derivative was going from negative region to positive region. Figure 4.10 to Figure 4.13 represent the curve fitted data of the four parameters and the first derivative of the curve fitted data for a randomly selected subject.

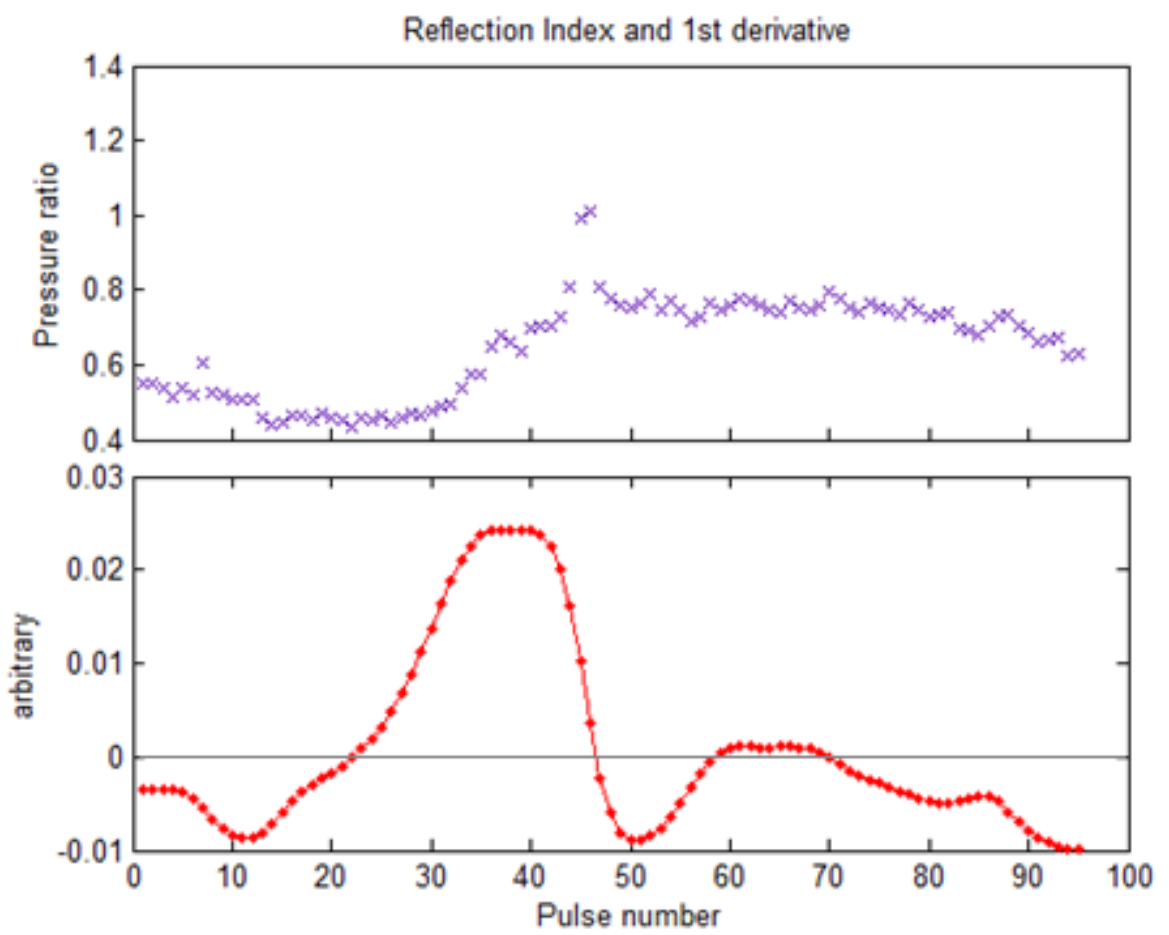

Figure 4.10: Smoothed Reflection Index Plot and First Derivative 

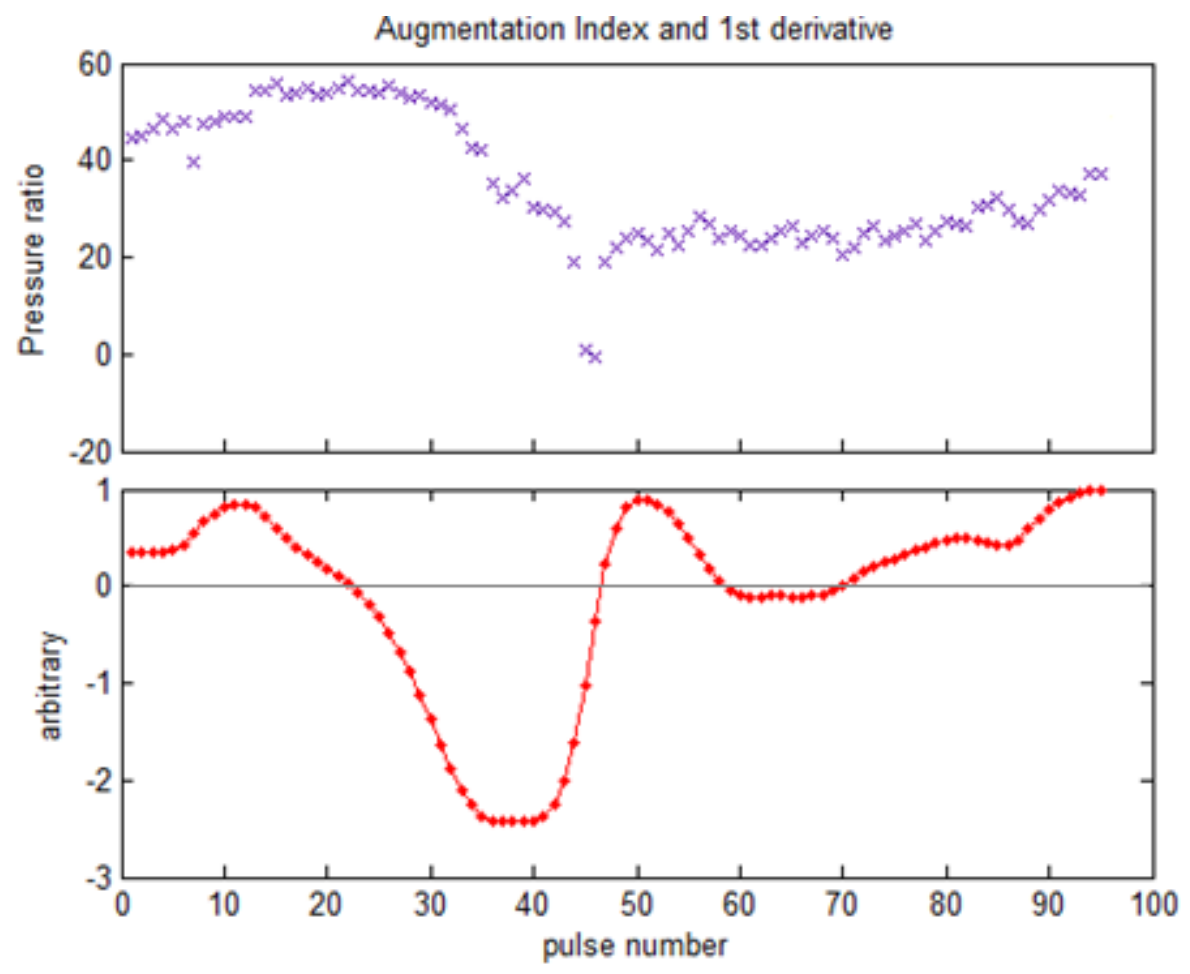

Figure 4.11: Smoothed Augmentation Index Plot and First Derivative
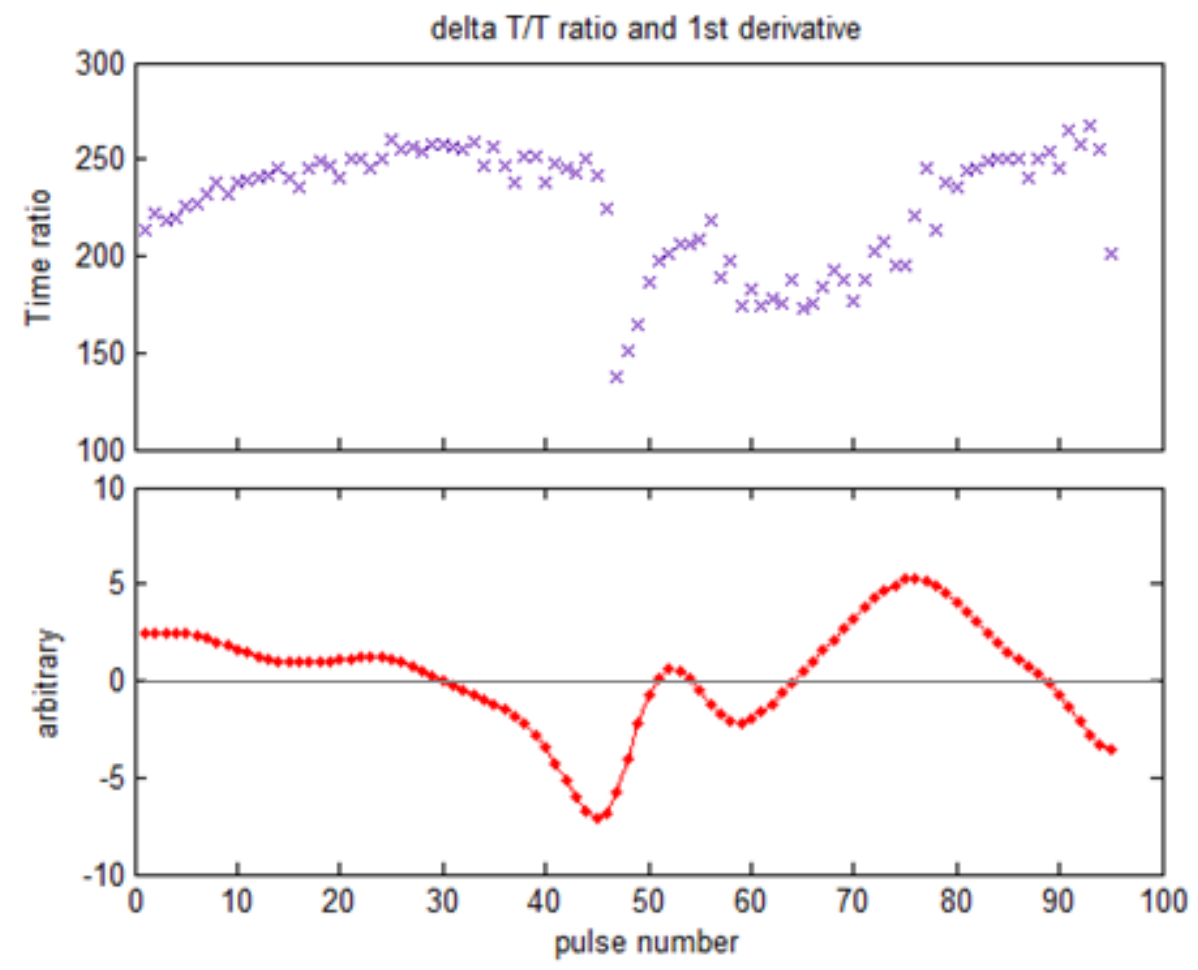

Figure 4.12: Smoothed $\Delta \mathrm{T} / \mathrm{T}$ ratio Plot and First Derivative 

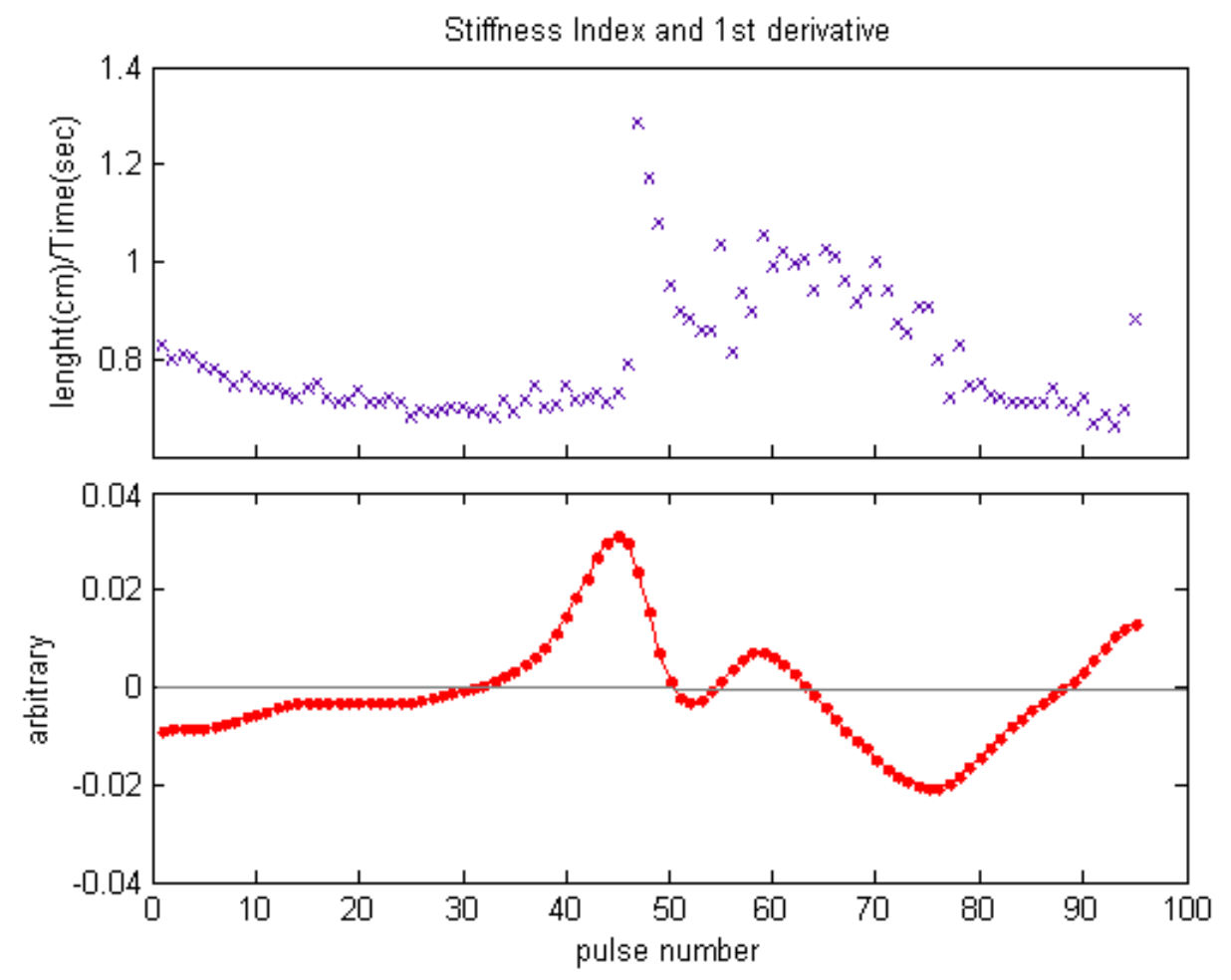

Figure 4.13: Smoothed Stiffness Index Plot and First Derivative

The Bland Altman plots constructed for SBP and DBP results obtained from curve fitting technique are presented in Figure 4.14 and Figure 4.15.

\subsection{First Derivative of the Pulse Waveform}

The first derivative of the pulse carries important information that can be used in pulse morphology and the proposed method of estimating BP based on pulse morphology. In cases that the dicrotic notch of the pulse waveform is less or not visible and the second peak cannot be detected, the first derivative of the pulse can lead us to find the location of the second peak for In elderly subjects due to the reducing of the flexibility of the large arteries, the dicrotic notch becomes less or not visible. Also, in Oscillometric cuff deflation waveform, by going toward 

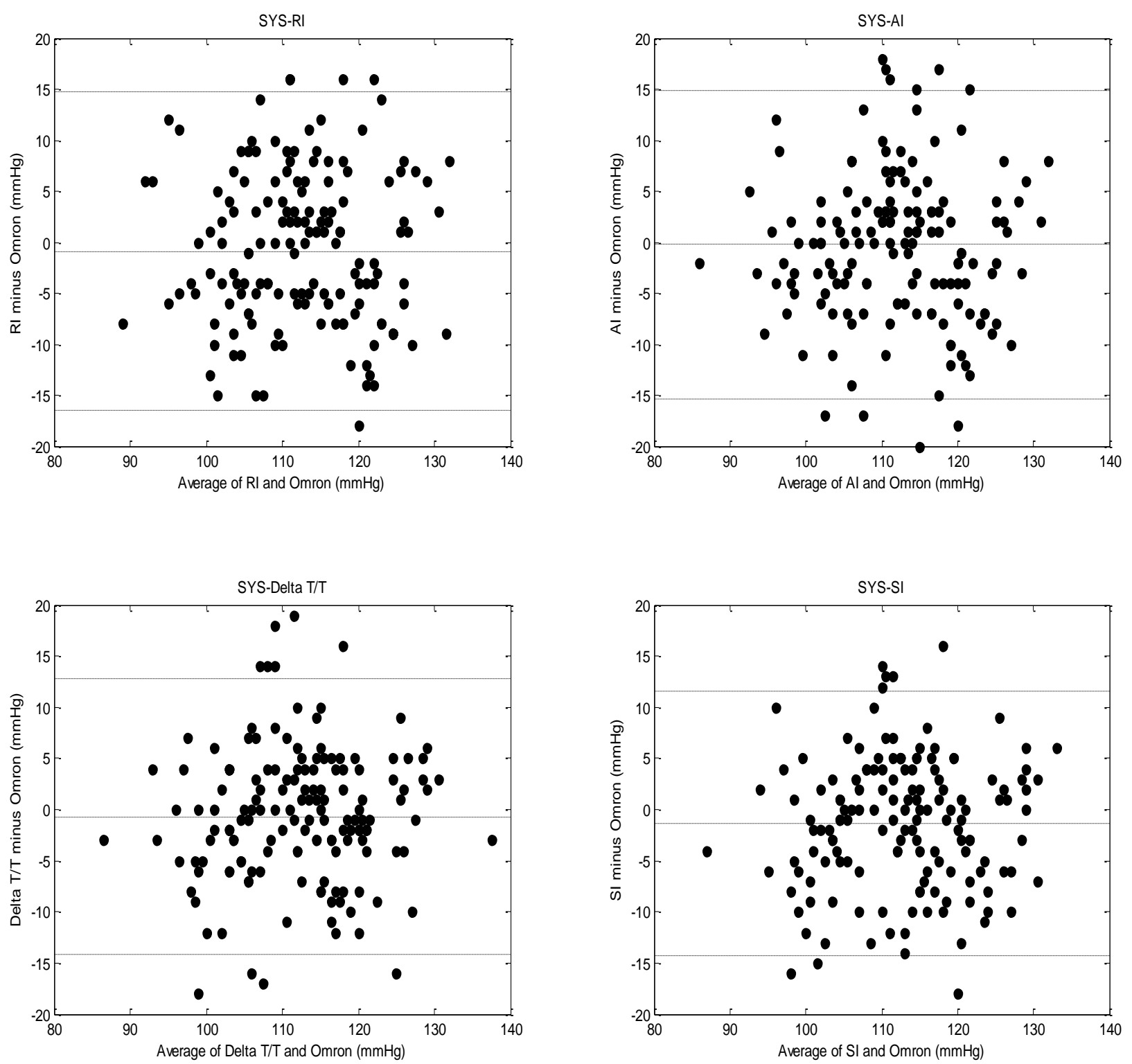

Figure 4.14: Bland Altman plot comparing the SBP Estimated from curve fitted graphs with OMRON results 

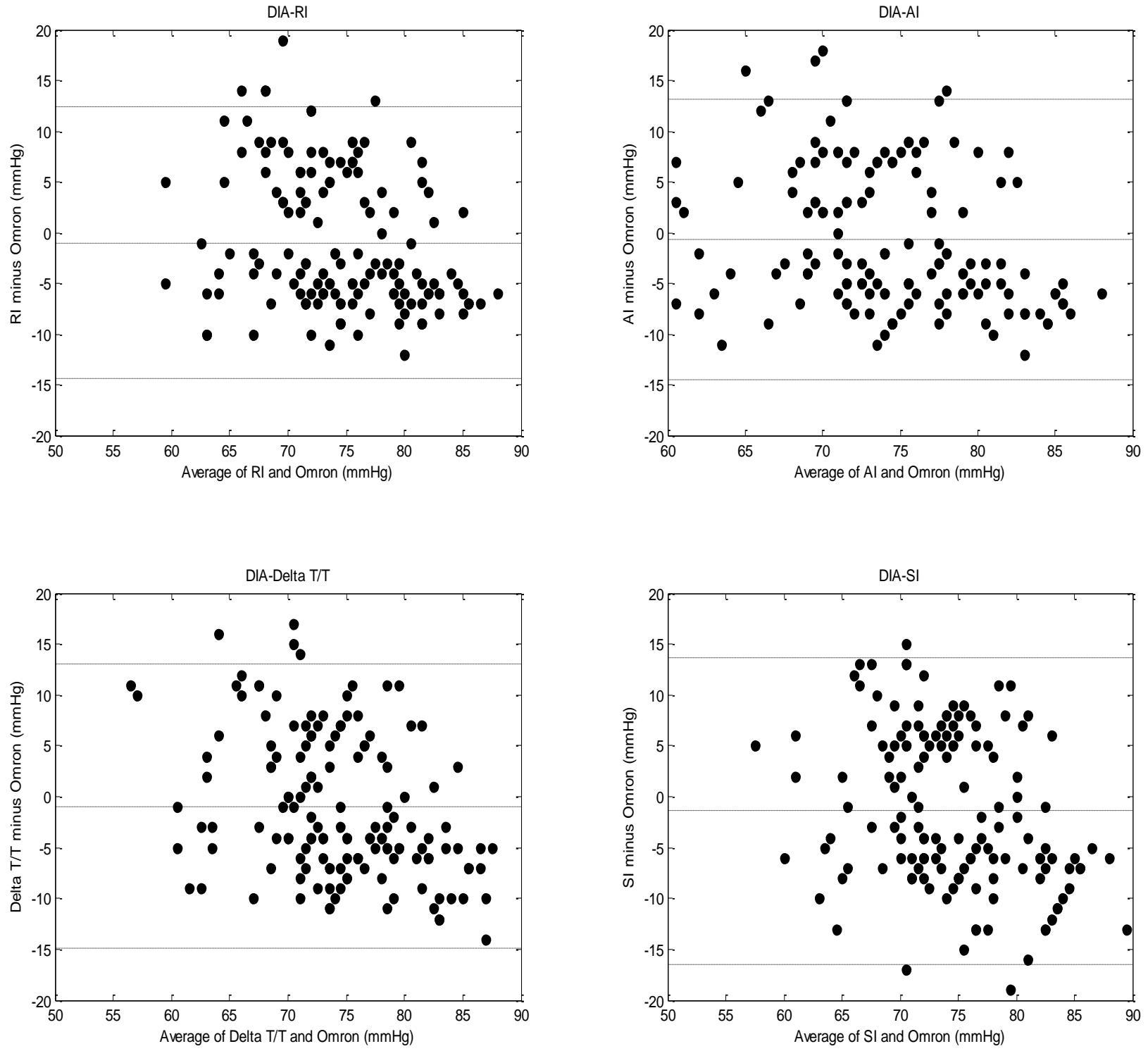

Figure 4.15:Bland Altman plot comparing the DBP Estimated from curve fitted graphs with OMRON results

further investigations. The maximum slope of the pulse can also be found from the first derivative of the pulse contour.

diastolic region the notch becomes less visible. In these cases, detecting the location of the second peak becomes an issue. To solve this problem, the first derivative of the pulse was taken (Figure 4.16). The second point that the signal of the first derivative goes from positive to negative regions can be mapped to the pulse waveform and considered to be the location of the 
second peak. The location of the peak with maximum amplitude on the first derivative signal can also be mapped to the pulse waveform to represent the location of the maximum slope in the blood pressure pulse waveform. The maximum slope can be also used to estimate BP, as described in Section 4.8.

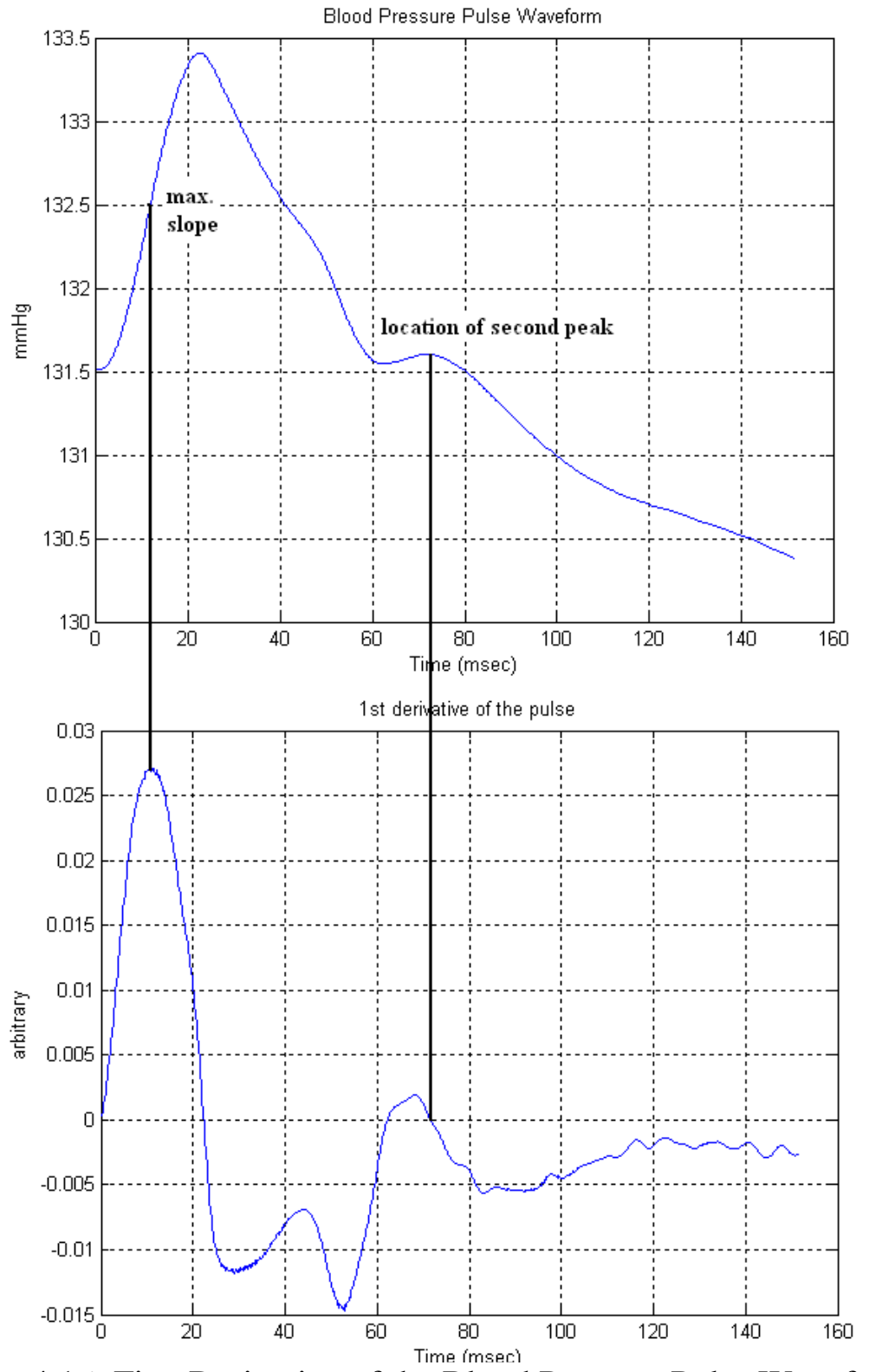

Figure 4.16: First Derivative of the Blood Pressure Pulse Waveform 


\subsection{Student's $\mathrm{t}$ test Comparison}

Student's t test is used to compare two sets of quantitative data when samples are collected independently of one another. This test is one of the most commonly used techniques for testing hypothesis on the basis of a difference between sample means. The test static is calculated using a formula as follow:

$$
t=\frac{\boldsymbol{X}-\mu}{s / \sqrt{n}}
$$

Where $\mathbf{X}$ is the sample mean, $\mu$ is the hypothesized population mean, $\mathbf{s}$ is the standard deviation, and $\mathrm{n}$ is the sample size. The nominator gets larger as the means get further apart and denominator gets smaller as the sample variances decrease or the sample sizes increase. Matlab performs a t test of the hypothesis that the data is a vector which comes from a distribution with mean zero and returns the result of the test in $\mathrm{H}$. $\mathrm{H}=0$ indicates that the null hypothesis cannot be rejected at the $5 \%$ significant level, and when $\mathrm{H}=1$ indicates that the null hypothesis can be rejected at the 5\% level. $\mathrm{P}$ is the probability, under the null hypothesis, of observing a value as extreme or more extreme of the test static. The BP estimation using the proposed method based on four parameters of the pulse waveforms and MAA method were compared using student's $t$ test. In this comparison each method was compared with OMRON results as reference and returned values from student's t test were compared. The $t$ test results of the proposed method with OMRON results and MAA method with OMRON results are presented in Tables 4.17 4.19. 
Table 4.17: Systolic Blood Pressure Student's t test

\begin{tabular}{|c|c|c|c|c|c|}
\hline & AI & SI & RI & $\Delta \mathrm{T} / \mathrm{T}$ & MAA \\
\hline $\begin{array}{c}\text { H at } 5 \% \\
\text { significant level }\end{array}$ & 0 & 0 & 0 & 0 & 0 \\
\hline P value & 0.9555 & 0.1332 & 0.0860 & 0.3728 & 0.9250 \\
\hline
\end{tabular}

Table 4.18: Mean Arterial Pressure Student's t test

\begin{tabular}{|c|c|c|c|c|c|}
\hline & AI & SI & RI & $\Delta \mathrm{T} / \mathrm{T}$ & MAA \\
\hline $\begin{array}{c}\text { H at } 5 \% \\
\text { significant level }\end{array}$ & 1 & 0 & 0 & 1 & 0 \\
\hline P value & $4.7344 \mathrm{e}-006$ & 0.7886 & 0.4824 & $2.3929 \mathrm{e}-005$ & 0.2572 \\
\hline
\end{tabular}

Table 4.19: Diastolic Blood Pressure Student's t test

\begin{tabular}{|c|c|c|c|c|c|}
\hline & AI & SI & RI & $\Delta \mathrm{T} / \mathrm{T}$ & MAA \\
\hline $\begin{array}{c}\text { H at } 5 \% \\
\text { significant level }\end{array}$ & 0 & 0 & 0 & 0 & 0 \\
\hline P value & 0.2400 & 0.1521 & 0.0629 & 0.0571 & 0.7282 \\
\hline
\end{tabular}

For 5\% significant level, $\mathrm{H}=1$ for $\mathrm{p}$ value being less than 0.05 . At MAP, BP estimation using AI and $\Delta \mathrm{T} / \mathrm{T}$ ratio showed to be more reasonable than other parameters and MAA. AT SBP and DBP none of the $\mathrm{P}$ values are less than 0.05 . However, RI at SBP and RI and $\Delta \mathrm{T} / \mathrm{T}$ ratio at DBP showed to be more reasonable than other parameters and MAA.

\subsection{Respiration in Blood Pressure}

As mentioned in chapter 2, Respiration can have influence on BP estimation. In some cases, the respiration effects can be large enough that estimated BP values cannot be truly representative of 
the subject's actual blood pressure. It is important to note that the term respiration is only used in literature because it is regarded as a chemical process. Instead, breathing is related to the ventilation movement of air in the lungs and diaphragm and is known as a physical process. Both chemical and physical processes have influenced BP but, some chemical processes that affect BP are unclear. Therefore, breathing is used in the work instead of respiration.

In this part of the thesis work, the proposed method of BP estimation using 4 parameters of pulse waveform compared to MAA method and OMRON in the cases of deep and fast breathings. 15 recordings with deep breathing and 15 recordings with fast breathing were taken and the proposed method of BP estimation and MAA were applied on them. Recordings were taken from five subjects. The subjects were asked to sit upright and perform fast and deep breathing at their own natural pace, with no outside control. Subject was asked to breathe normally during OMRON recording and results were used as reference. Subject was asked to breathe either fast or deep during the recording with the prototype device.

An example of cuff deflation waveform with fast breathing is shown in Figure 4.17. Figure 4.18 to Figure 4.20 show examples of three pulses at systolic, MAP, and diastolic regions respectively. Figure 4.21 to Figure 4.24 show the Bland-Altman plots, comparing the effect of fast breathing on the presented method of blood pressure estimation and MAA method at SBP, MAP, and DBP. An example of cuff deflation waveform with deep breathing is presented in Figure 4.25. Nine pulses at systolic, MAP, and diastolic regions are shown in Figure 4.26 to Figure 4.28 respectively. Figure 4.29 to Figure 4.32 show the Bland-Altman plots, comparing the effect of deep breathing on the presented method of blood pressure estimation and MAA method at SBP, MAP, and DBP. 


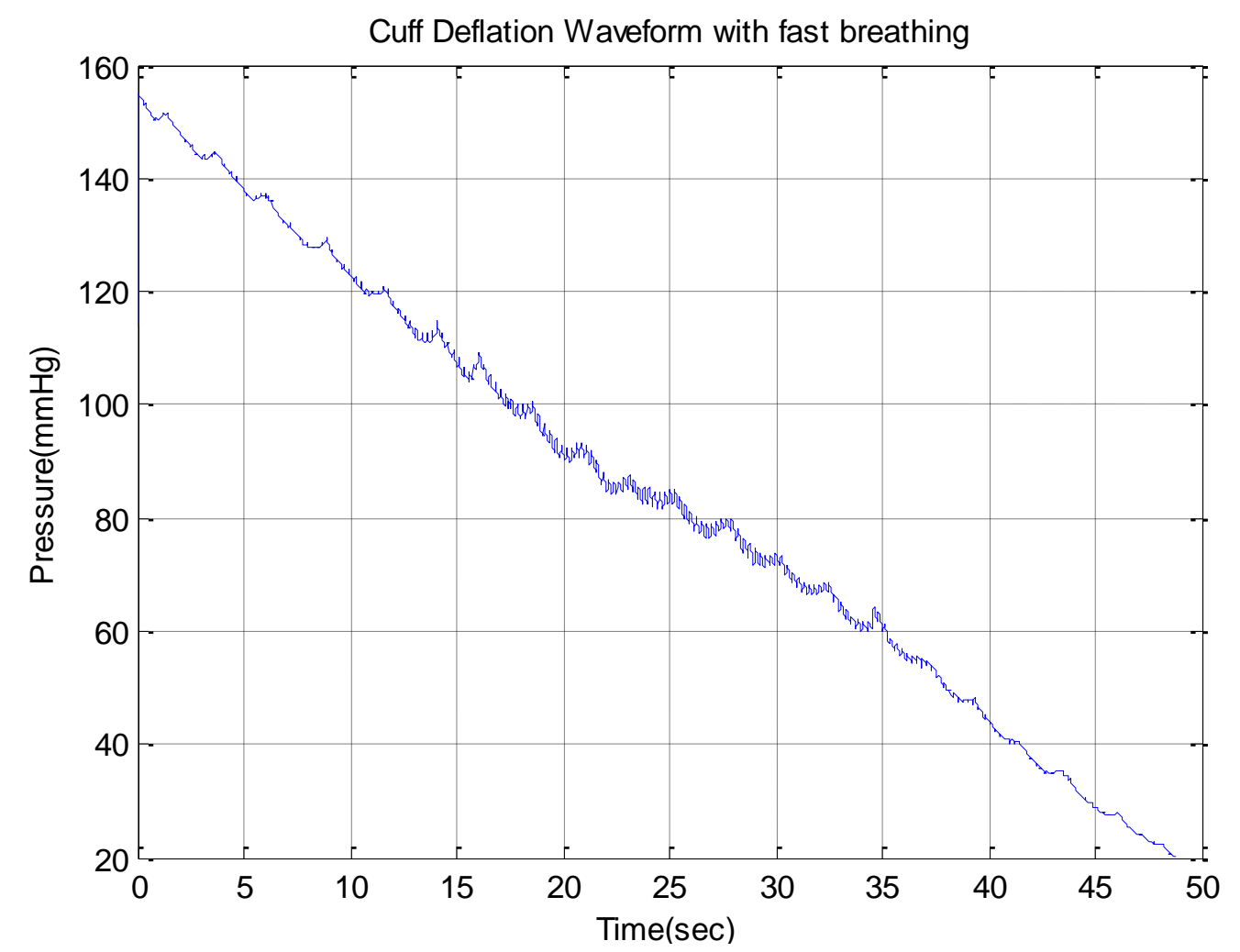

Figure 4.17: Cuff Deflation Waveform with Fast Breathing

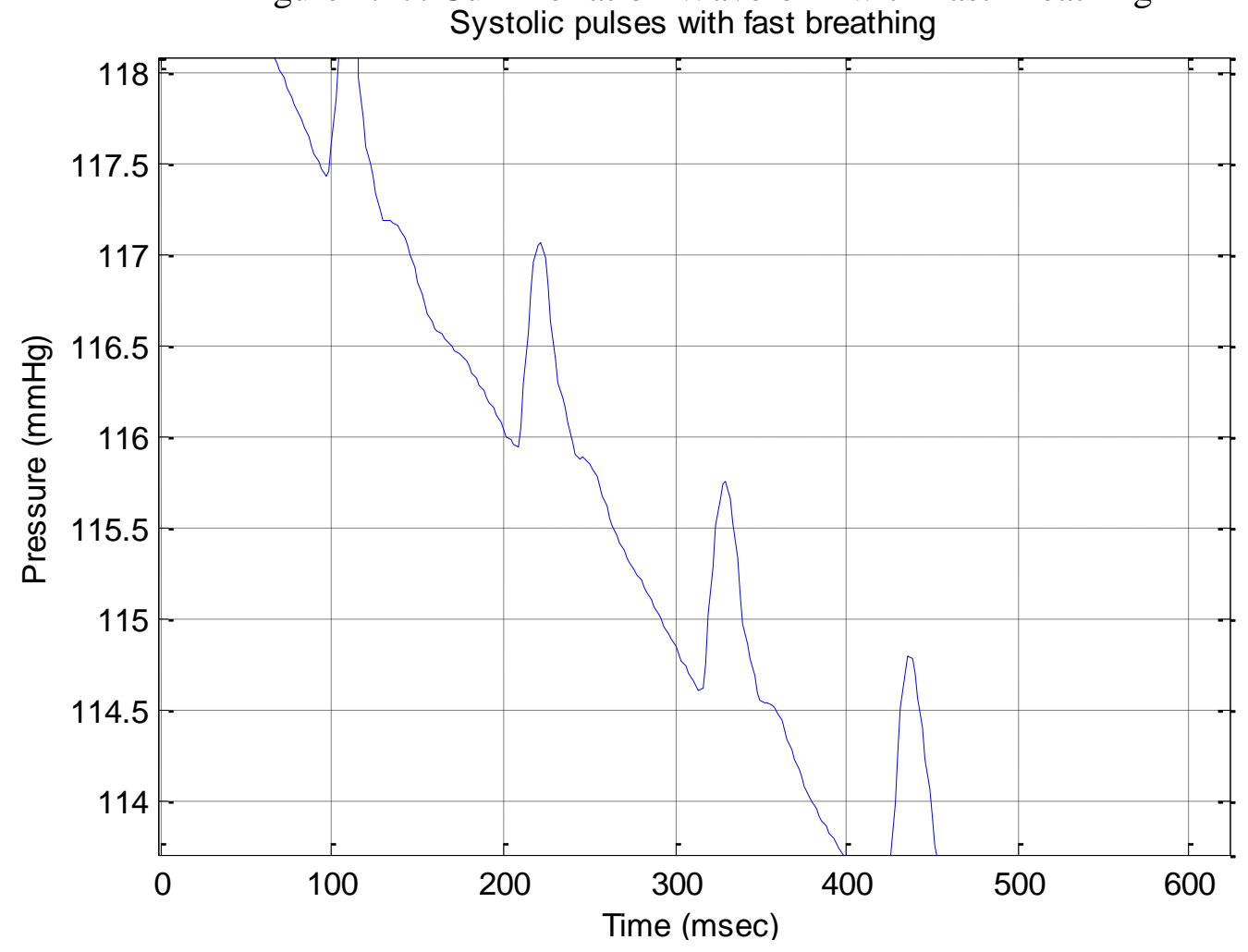

Figure 4.18: Pulses at Systolic region with Fast Breathing 


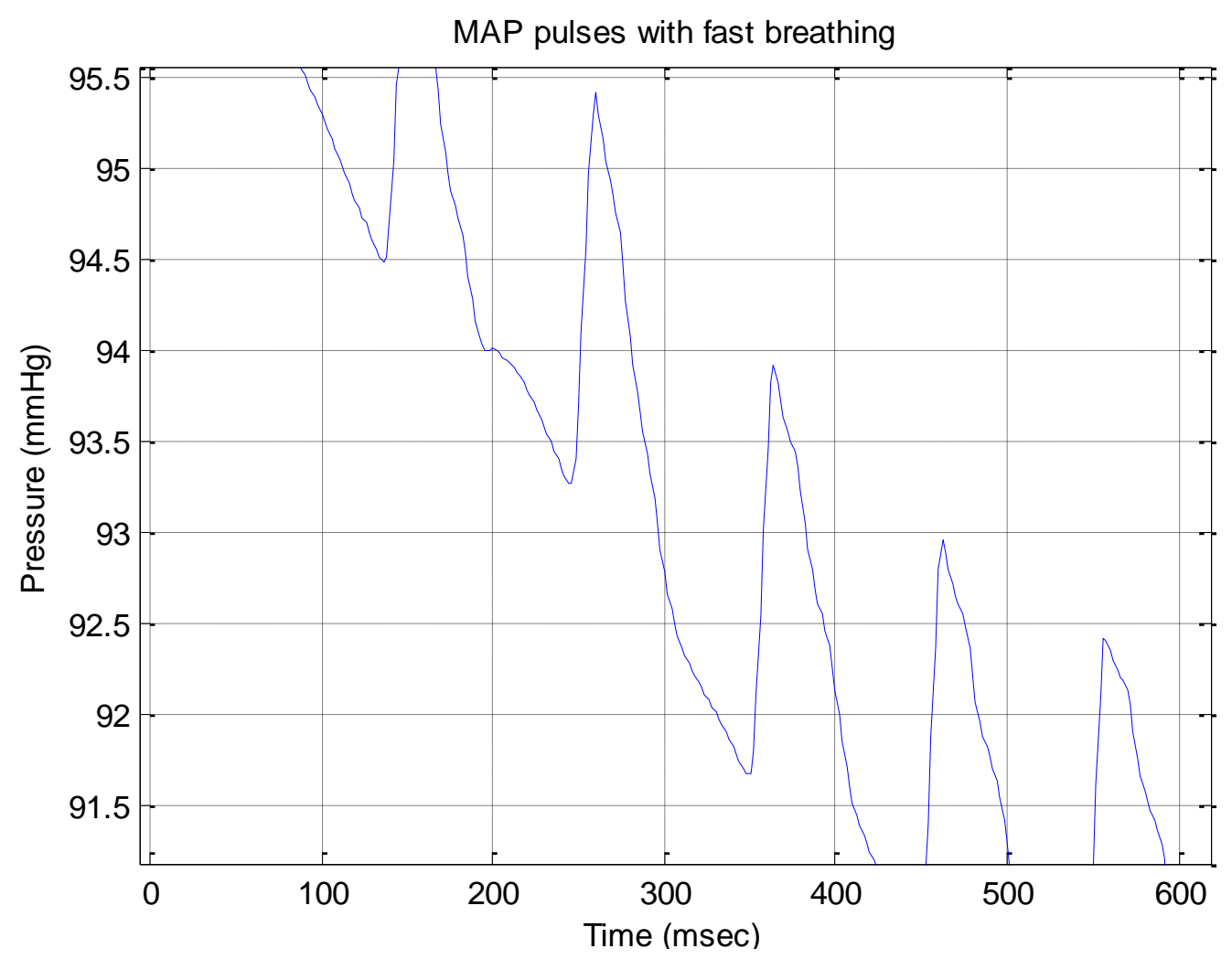

Figure 4.19: Pulses at MAP region with Fast Breathing

Diastolic pulses with fast breathing

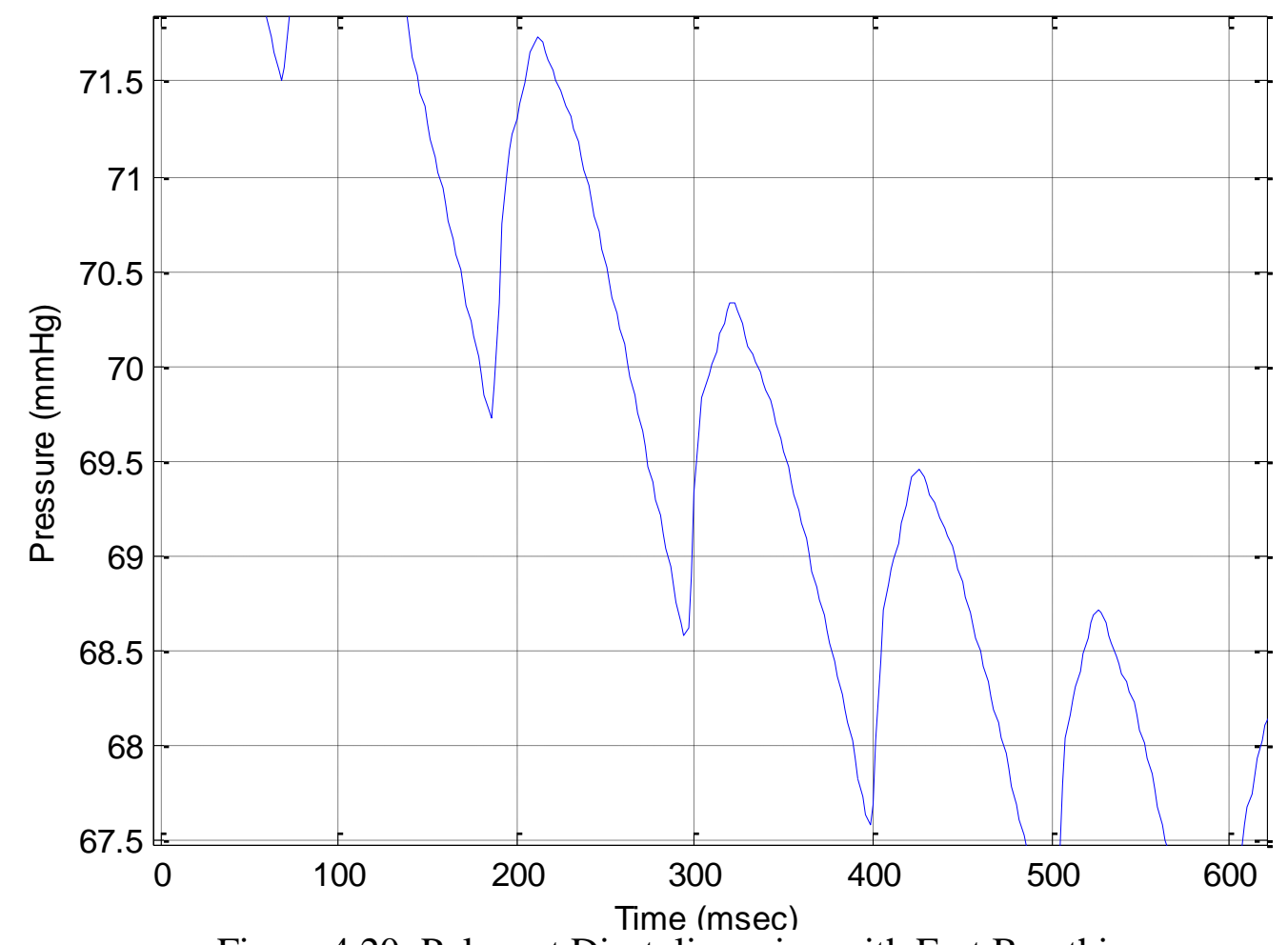

Figure 4.20: Pulses at Diastolic region with Fast Breathing 

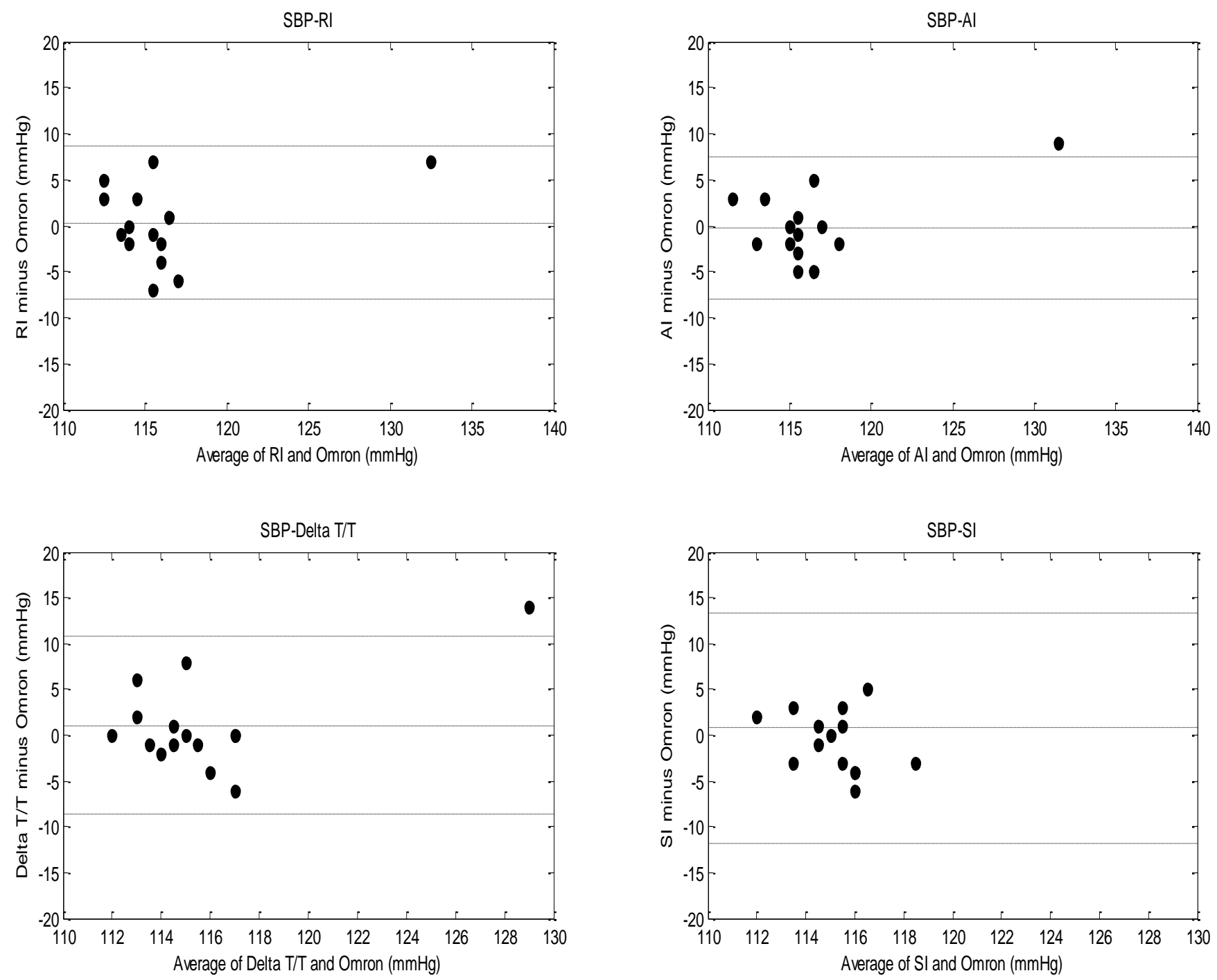

Figure 4.21: Comparison of Proposed Method with Reference Results under Fast Breathing at SBP 

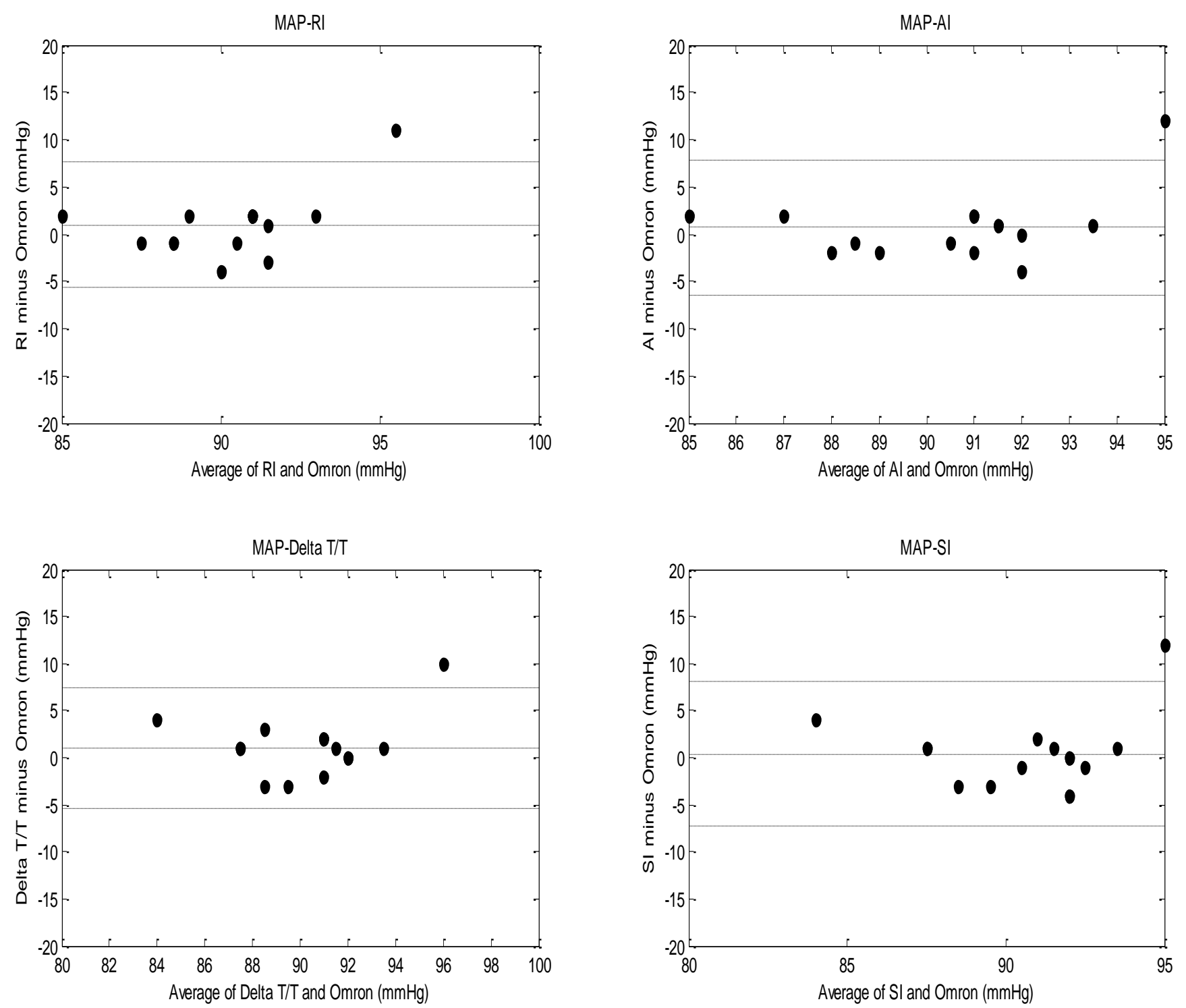

Figure 4.22: Comparison of Proposed Method with Reference Results under Fast Breathing at MAP 

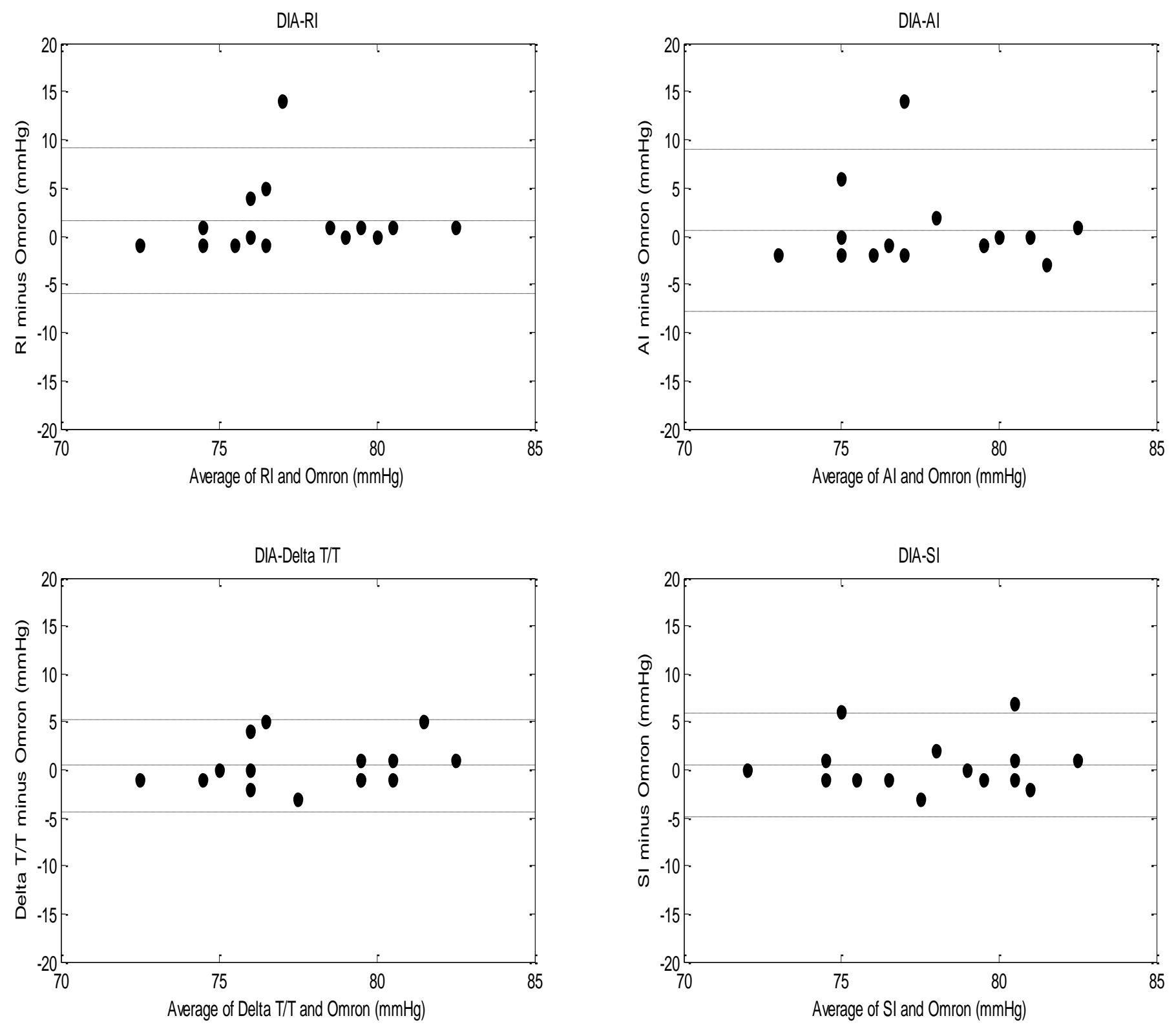

Figure 4.23: Comparison of the Proposed Method with Reference Results under Fast Breathing at DBP 

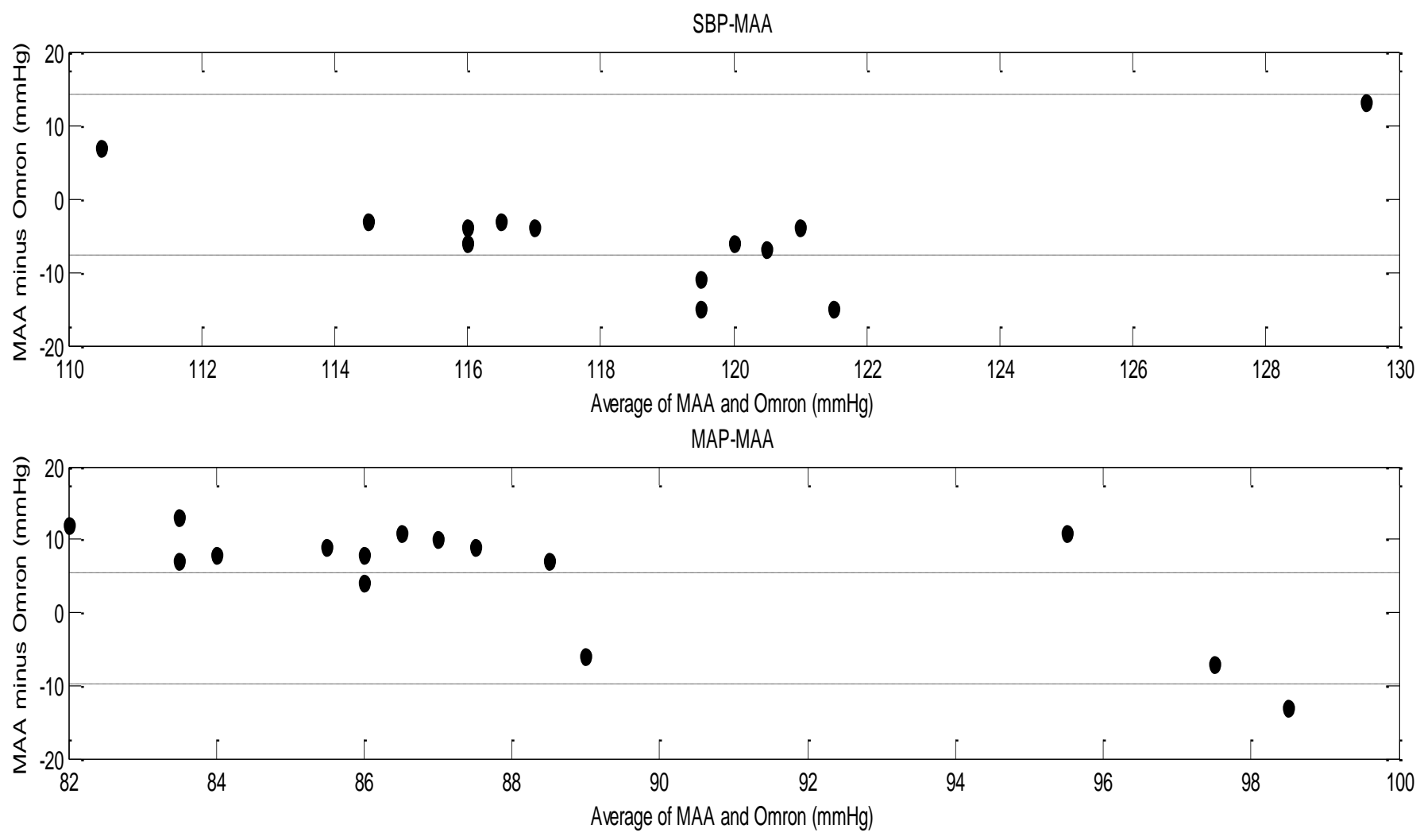

DBP-MAA

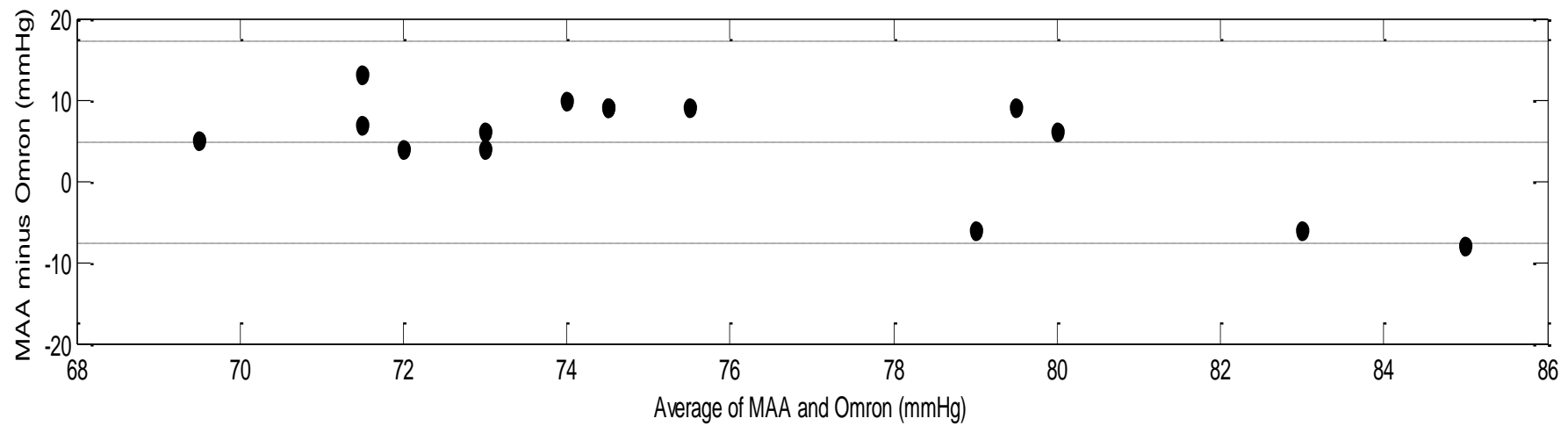

Figure 4.24: Comparison of MAA with Reference Results under Fast Breathing at SBP, MAP, and DBP 


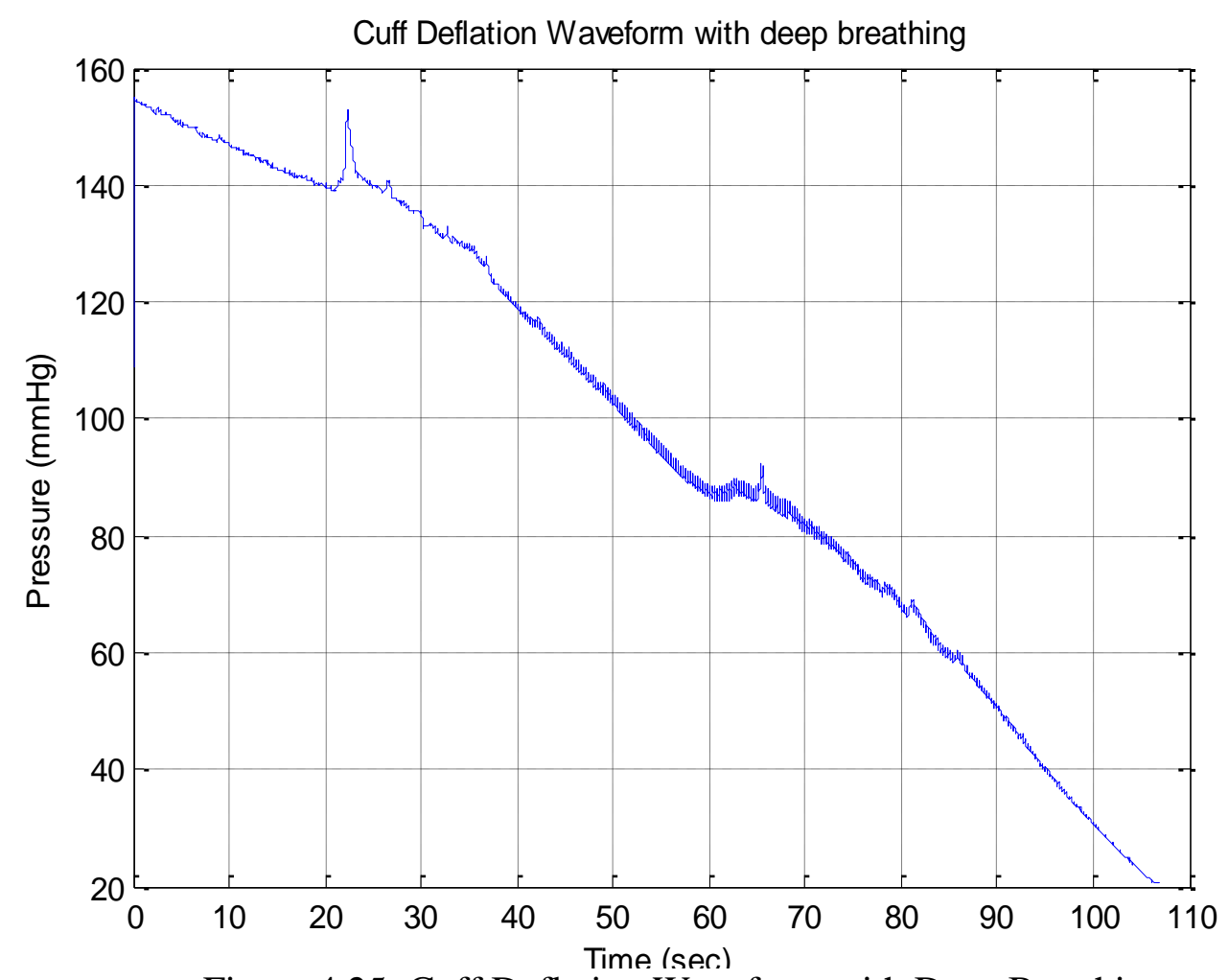

Figure 4.25: Cuff Deflation Waveform with Deep Breathing

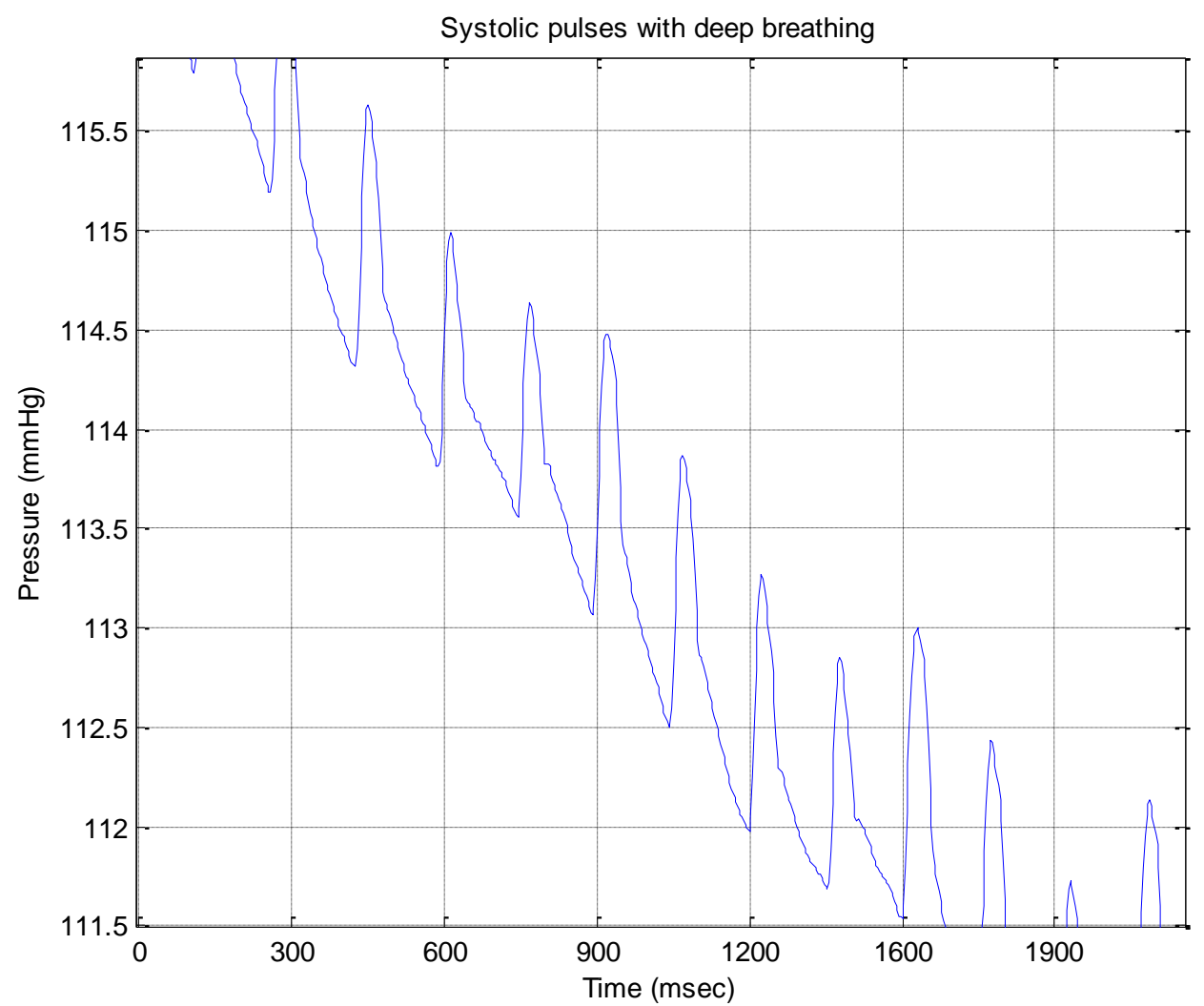

Figure 4.26: Pulses at Systolic region with Deep Breathing 


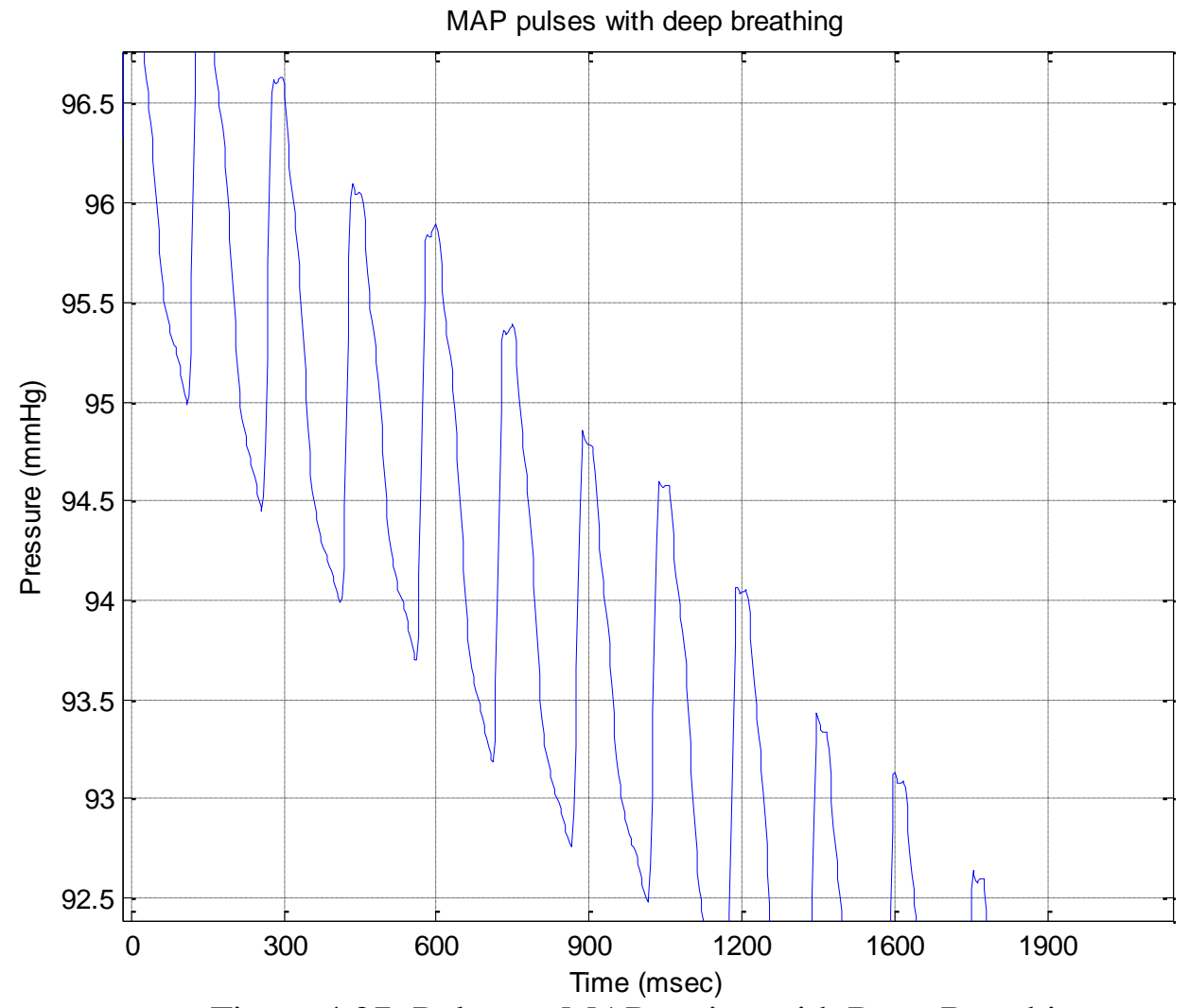

Figure 4.27: Pulses at MAP region with Deep Breathing

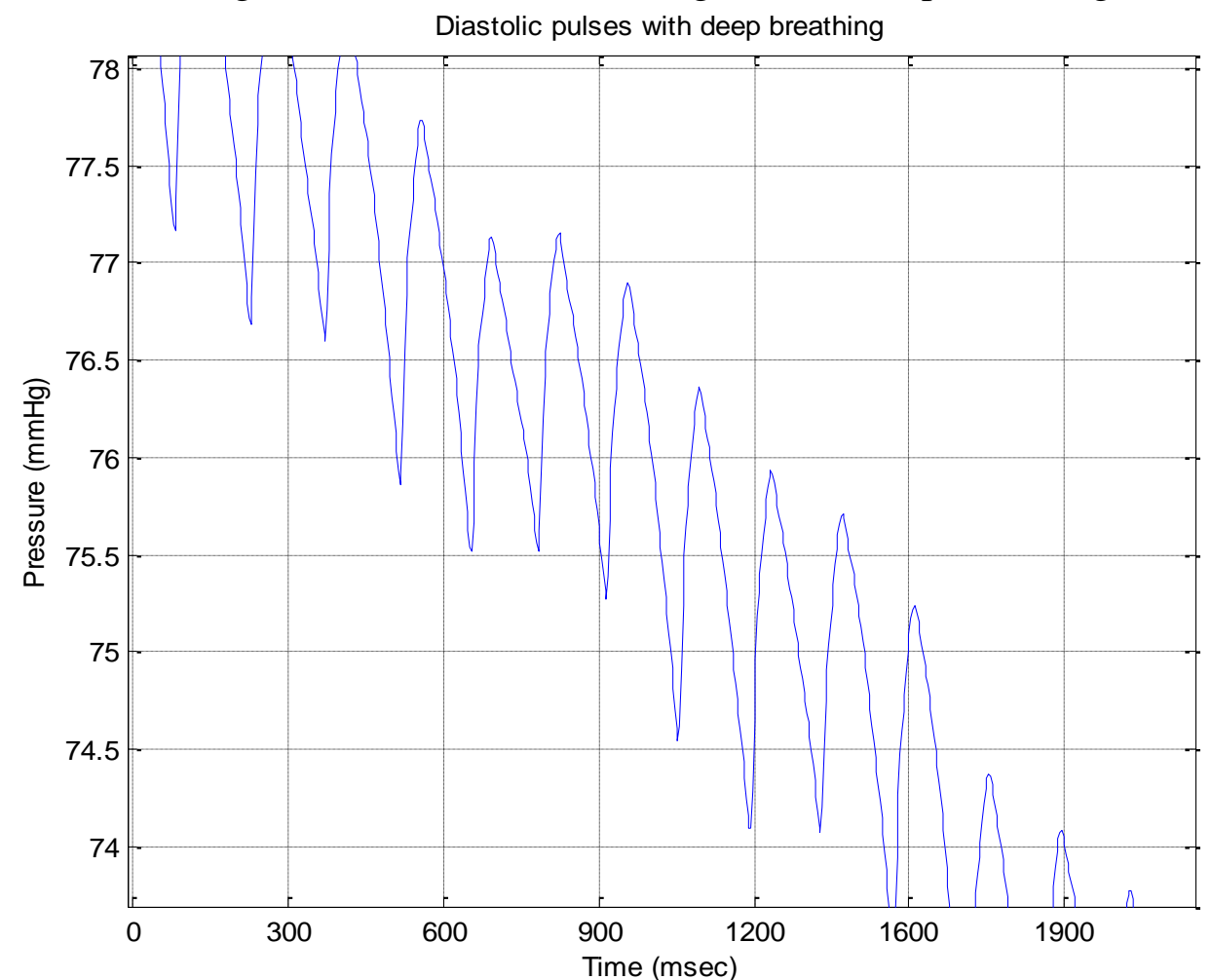

Figure 4.28: Pulses at Diastolic region with Deep Breathing 

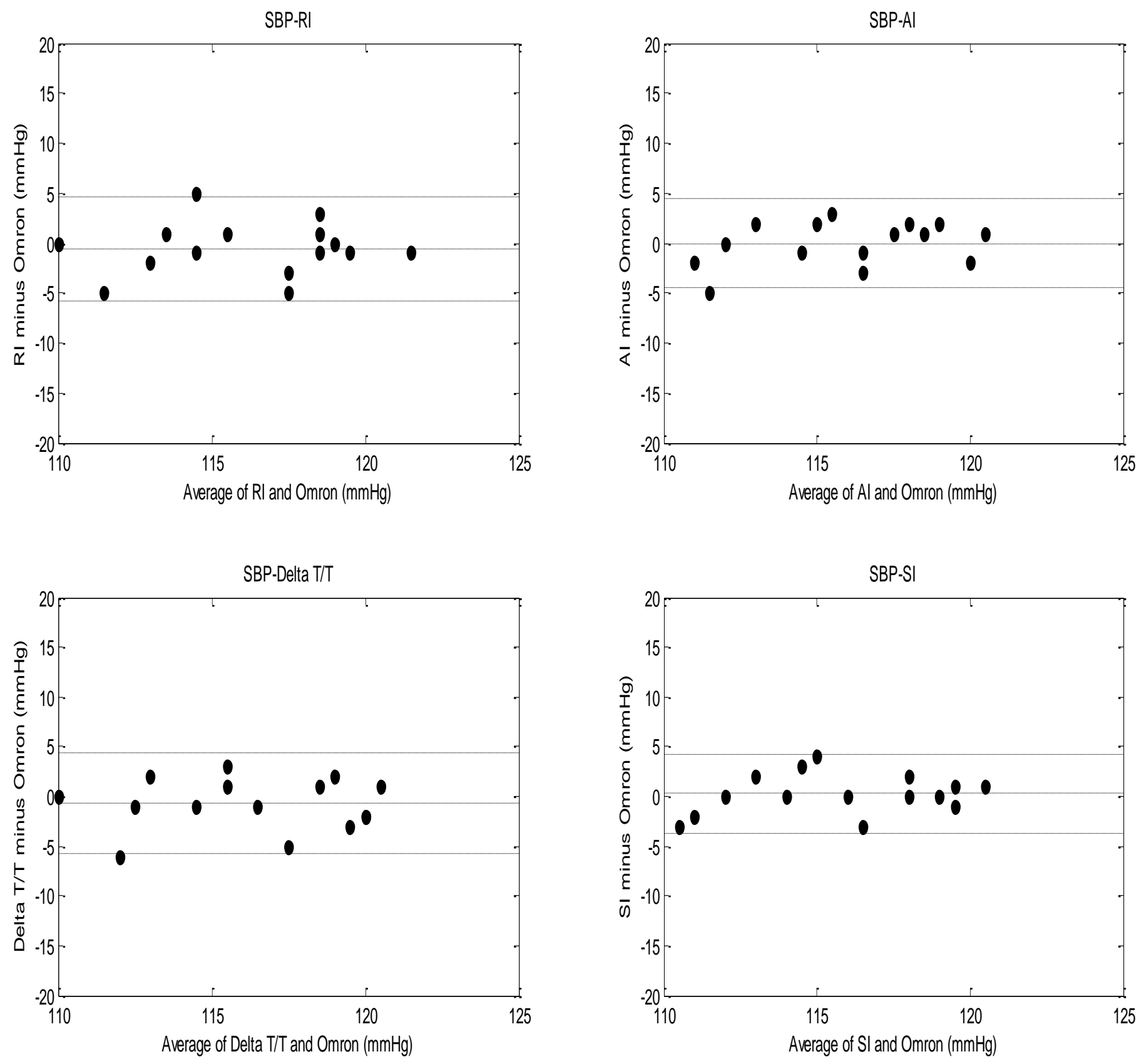

Figure 4.29: Comparison of Presented Method with Reference Results under Deep Breathing at

\section{SBP}



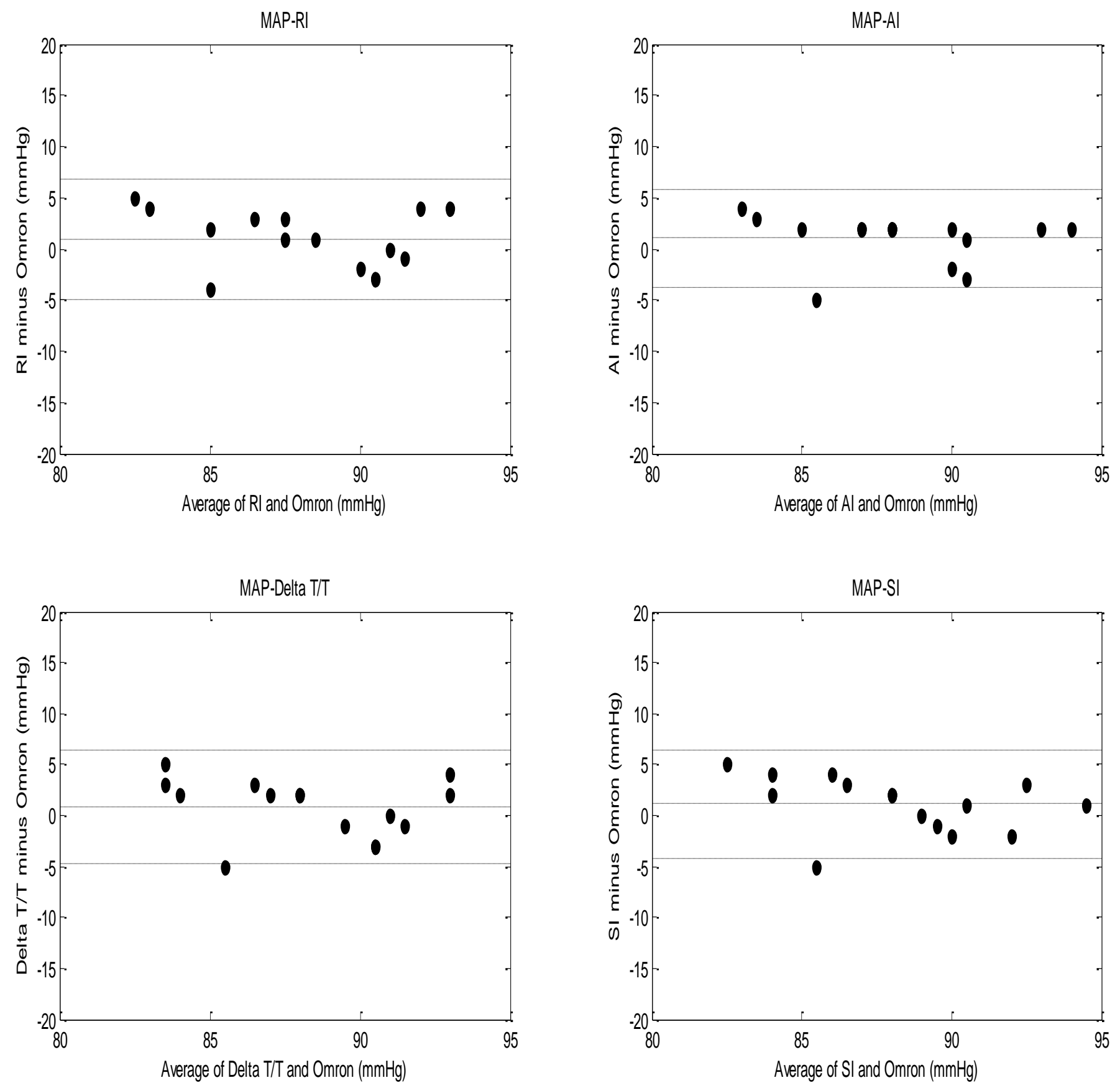

Figure 4.30: Comparison of Proposed Method with Reference Results under Deep Breathing at MAP 

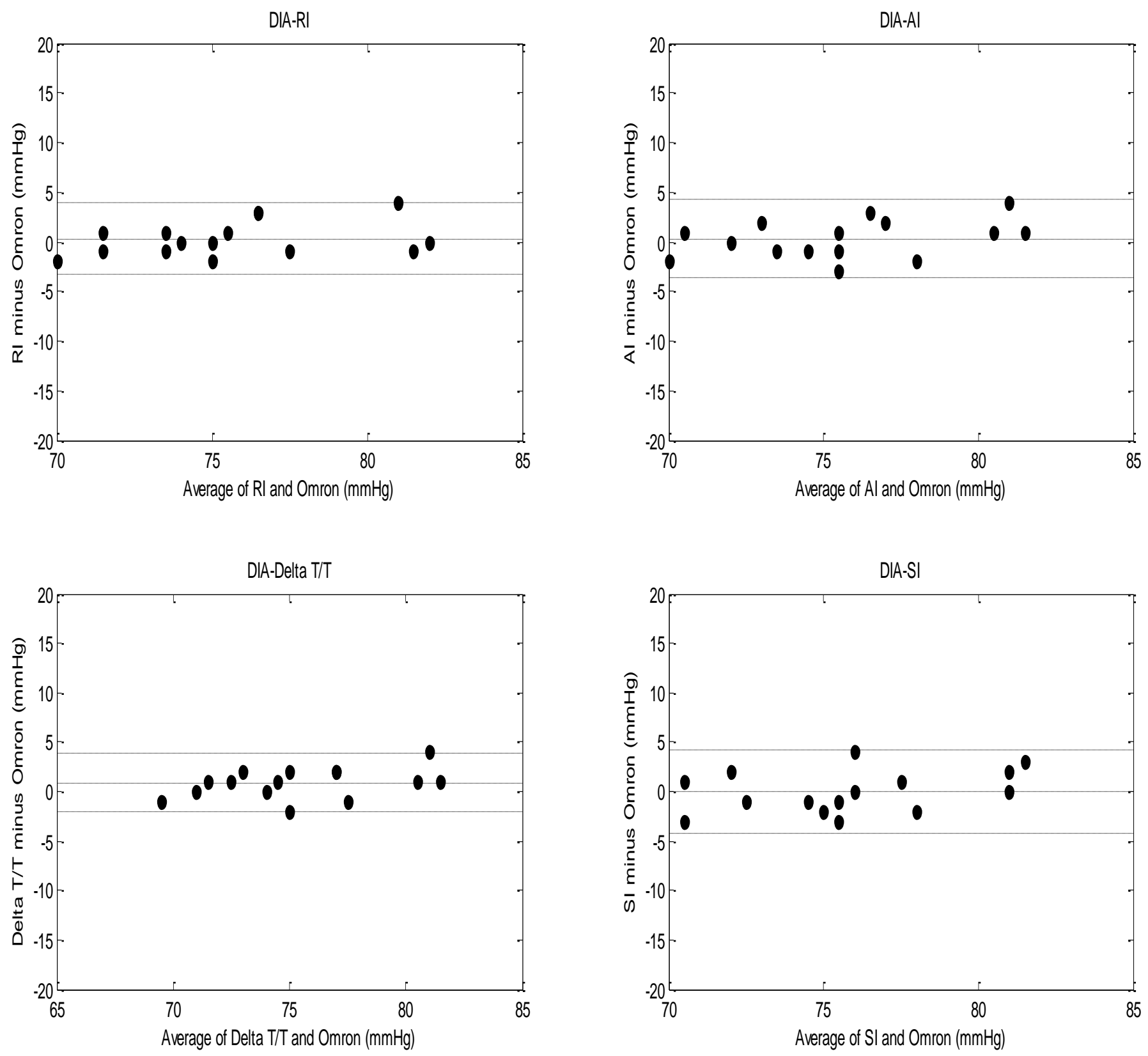

Figure 4.31: Comparison of Presented Method with Reference Results under Deep Breathing at DBP 

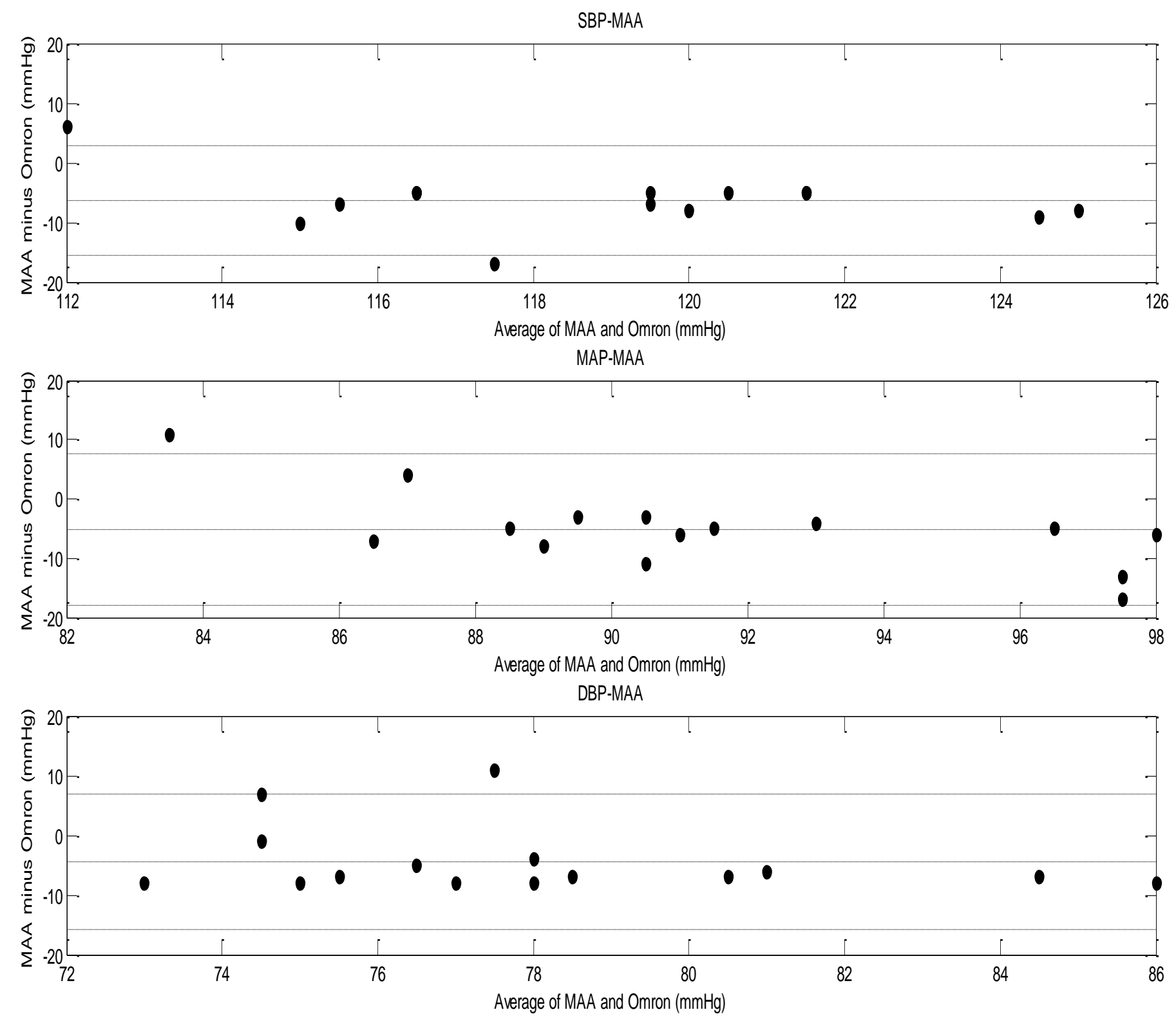

Figure 4.32: Comparison of MAA with Reference Results under Deep Breathing at SBP, MAP, and DBP 


\subsection{Blood Pressure Estimation using Maximum Slope}

Maximum slope of the blood pressure pulse waveform can also be used to estimate BP. The location of maximum slope can be determined from the first derivative of the waveform as described in Section 4.4. In this part of the thesis work, the maximum slopes of all pulses from cuff deflation pressure waveform were computed and plotted (Figure 4.33). After curve fitting the graphs (Figure 4.34), an envelope was achieved. The same technique as MAA method was used to estimate BP from the detected envelope (Figure 4.35). The maximum point was considered to represent MAP (OPI) pulse. Similar to other Oscillometric methods of blood pressure estimation, the left side of MAP (OPI) is called systolic region and the right side is diastolic region. Again, systolic region and diastolic region does not have any physiological meaning. They just show that the SBP and DBP are located in their own regions.

After detecting MAP pulse from envelope, the systolic and diastolic pulses will be obtained by their corresponding systolic and diastolic ratios. The systolic ratio used in this technique is 0.75 and diastolic ratio is 0.5 . Systolic and diastolic ratios can be found by least squares optimization. The least square error in blood pressure can be expressed as:

$$
\begin{aligned}
\operatorname{Err}_{S B P} & =\sum_{i=1}^{175}\left[S B P_{i}-s_{i}\left(r_{s}\right)\right]^{2} \\
\operatorname{Err}_{D B P} & =\sum_{i=1}^{175}\left[D B P_{i}-d_{i}\left(r_{d}\right)\right]^{2}
\end{aligned}
$$

Where $\mathrm{i}$ is the number of recordings, $\mathrm{s}_{\mathrm{i}}\left(\mathrm{r}_{\mathrm{s}}\right)$ is the function that returns SBP for a given recording with input systolic ratio $\left(r_{s}\right)$ and $d_{i}\left(r_{d}\right)$ function does the same thing for DBP. The goal is to have systolic and diastolic ratios that can minimize the systolic and diastolic error. Functions $\mathrm{s}_{\mathrm{i}}\left(\mathrm{r}_{\mathrm{s}}\right)$ and $d_{i}\left(r_{d}\right)$ are very complicated to drive. Therefore, the ratios which produce the minimum least 
square error are found by computing the error empirically for a predefined set of ratios. Using a set of ratios can provide a set of errors. The minimum error in the set can be determined and the corresponding ratio is taken as the desired ratio.

An advantage of estimating BP using maximum slope over MAA is that this technique is less sensitive to noise [78]. In MAA method, maximum amplitude (first peak) of the pulse waveforms is used to detect the envelope while random noise with higher amplitude can be detected instead of the first peak and affect the shape of the envelope which would lead to source of error in BP estimation. However, unpredictable noise has less effect on maximum slope of the waveform and the detected envelope and BP estimation using maximum slope can be more robust.

The comparison of BP estimation using maximum slope with MAA method and OMRON results is shown in Table 4.20 to Table 4.22. The student's t test results of BP estimation using maximum slope and MAA compared to OMRON results are presented in Table 4.23. The Bland Altman plots are also constructed for SBP, MAP, and DBP estimated with the maximum slopes and OMRON recording shown in Figure 4.36 to Figure 4.38. Comparison of all trials of all 18 subjects with MAA and OMRON are presented in Appendix B. 


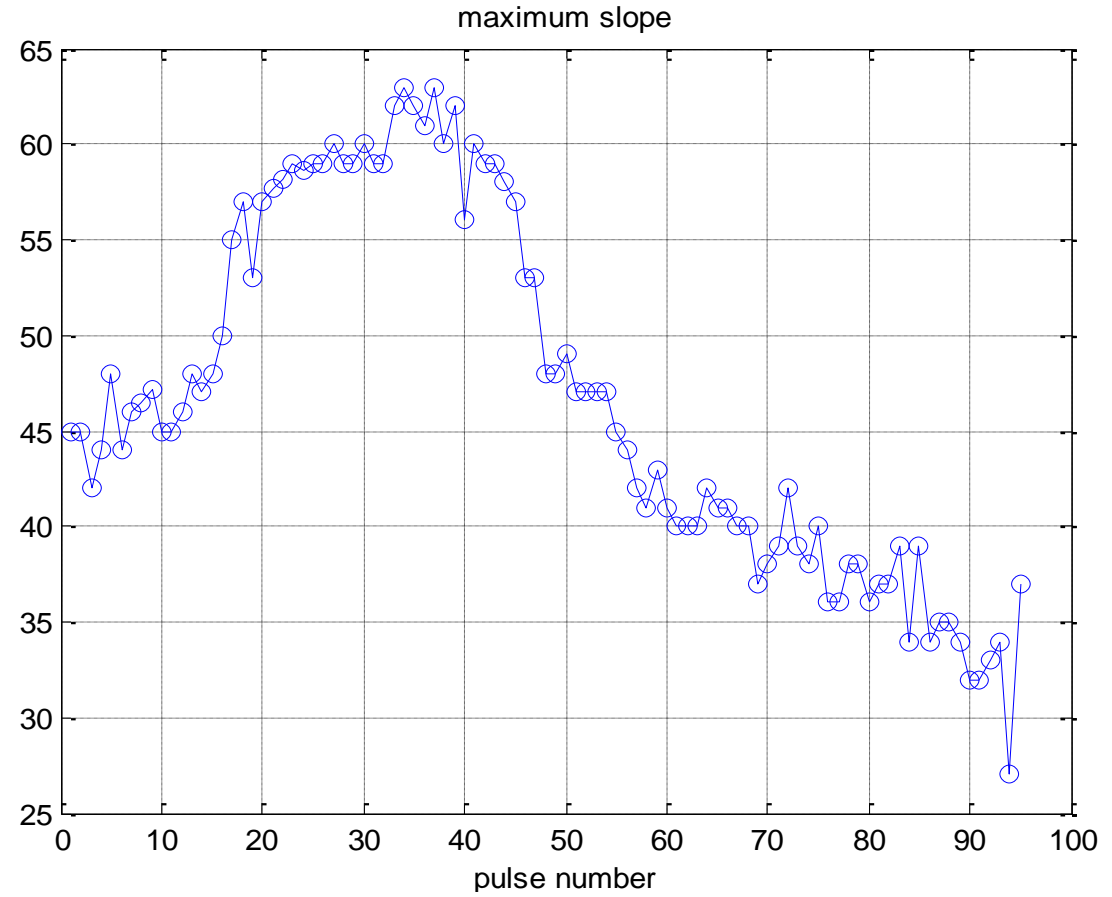

Figure 4.33: Plot of Maximum Slope of all Pulses

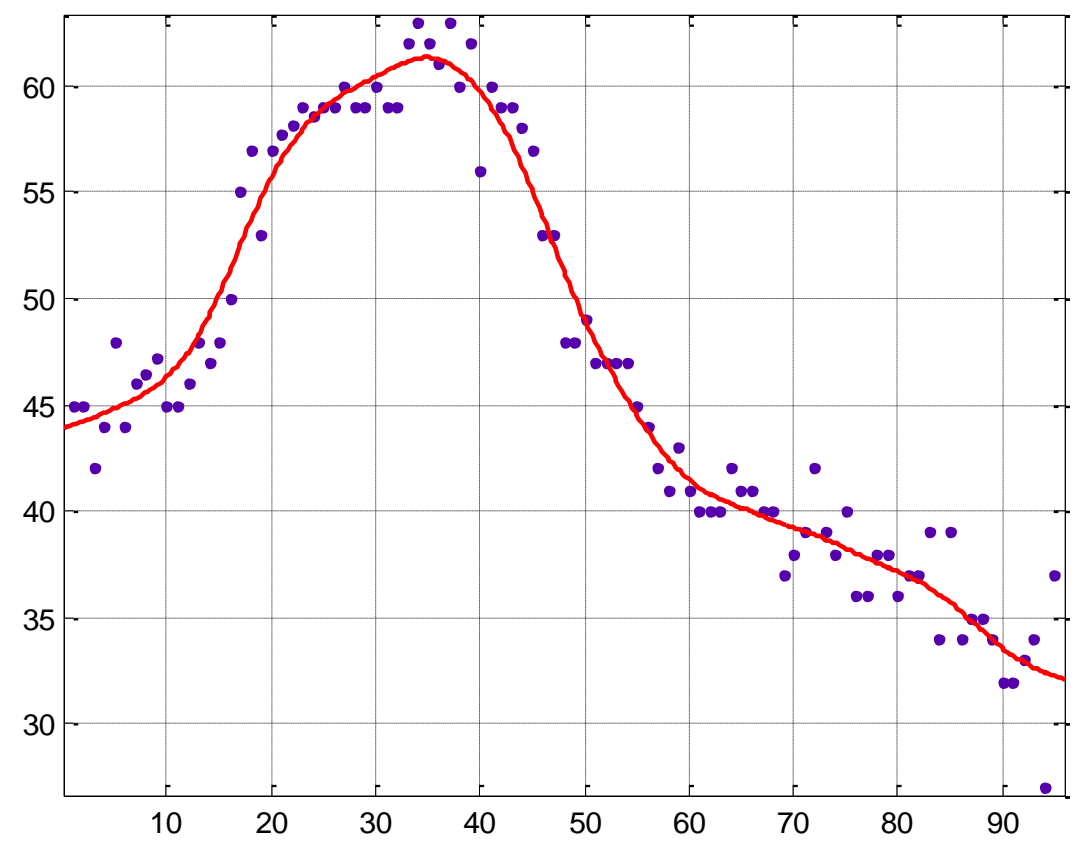

Figure 4.34: The curve fitted of Maximum Slope plot 


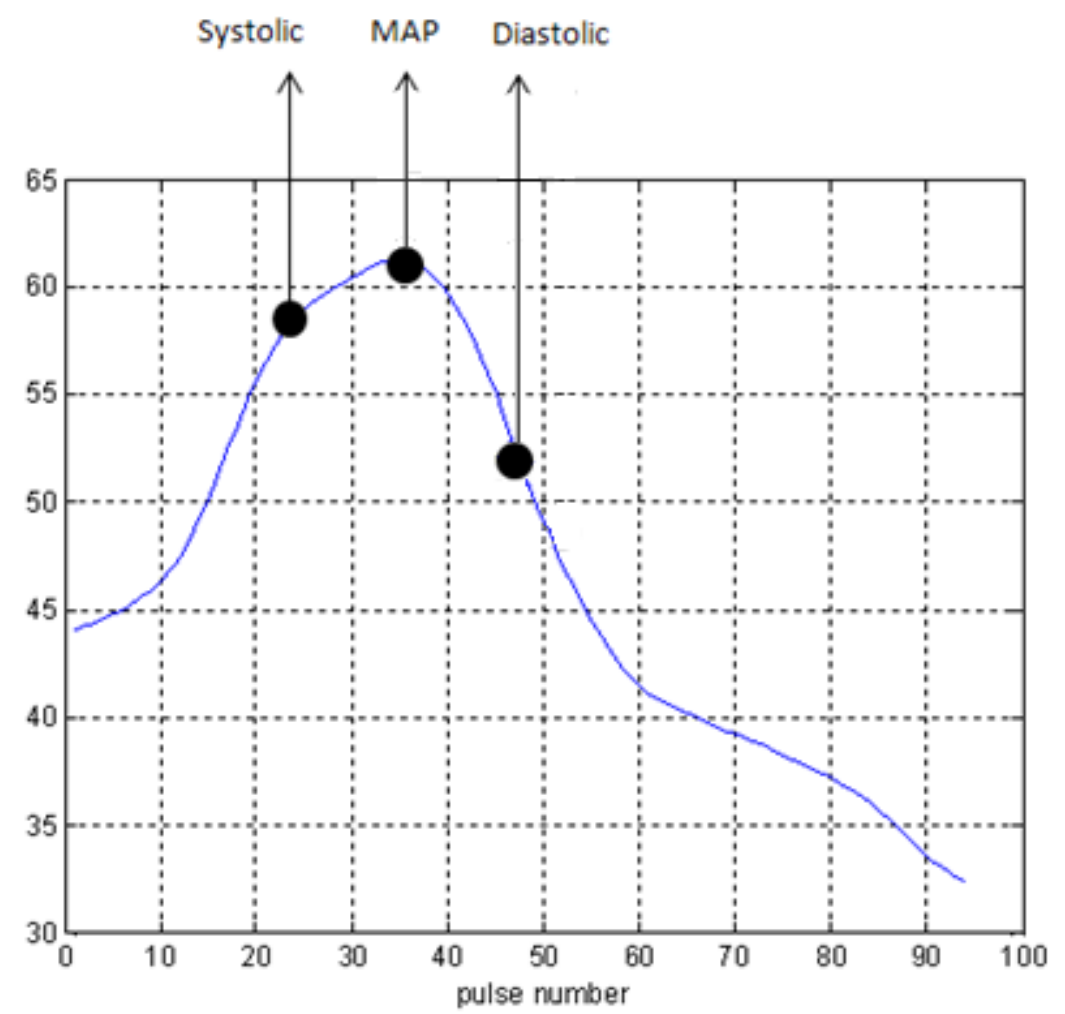

Figure 4.35: BP Estimation from Maximum Slope method

Table 4.20: Comparison of Max. Slope method with MAA and OMRON results in SBP

\begin{tabular}{|c|c|c|c|}
\hline SBP & Maximum Slope & MAA & OMRON \\
\hline $\mathrm{mmHg}$ & $112.53 \pm 10.15$ & $114.06 \pm 6.7$ & $114.13 \pm 9.2$ \\
\hline
\end{tabular}

Table 4.21: Comparison of Max. Slope method with MAA and OMRON results in MAP

\begin{tabular}{|c|c|c|c|}
\hline MAP & Maximum Slope & MAA & OMRON \\
\hline $\mathrm{mmHg}$ & $85.83 \pm 7.2$ & $87.64 \pm 4.73$ & $87 \pm 5.42$ \\
\hline
\end{tabular}


Table 4.22: Comparison of Max. Slope method with MAA and OMRON results in DBP

\begin{tabular}{|c|c|c|c|}
\hline DBP & Maximum Slope & MAA & OMRON \\
\hline $\mathrm{mmHg}$ & $72.81 \pm 6.95$ & $72.82 \pm 8.7$ & $72.52 \pm 4.8$ \\
\hline
\end{tabular}

Table 4.23: Maximum Slope Student's t test

\begin{tabular}{|c|c|c|c|c|c|c|}
\hline & \multicolumn{3}{|c|}{ Maximum Slope } & \multicolumn{3}{c|}{ MAA } \\
\cline { 2 - 7 } & SBP & MAP & DBP & SBP & MAP & DBP \\
\hline $\begin{array}{c}\text { H at 5\% } \\
\text { significant level }\end{array}$ & 1 & 0 & 0 & 0 & 0 & 0 \\
\hline P value & 0.0491 & 0.2230 & 0.0615 & 0.3657 & 0.4610 & 0.1960 \\
\hline
\end{tabular}

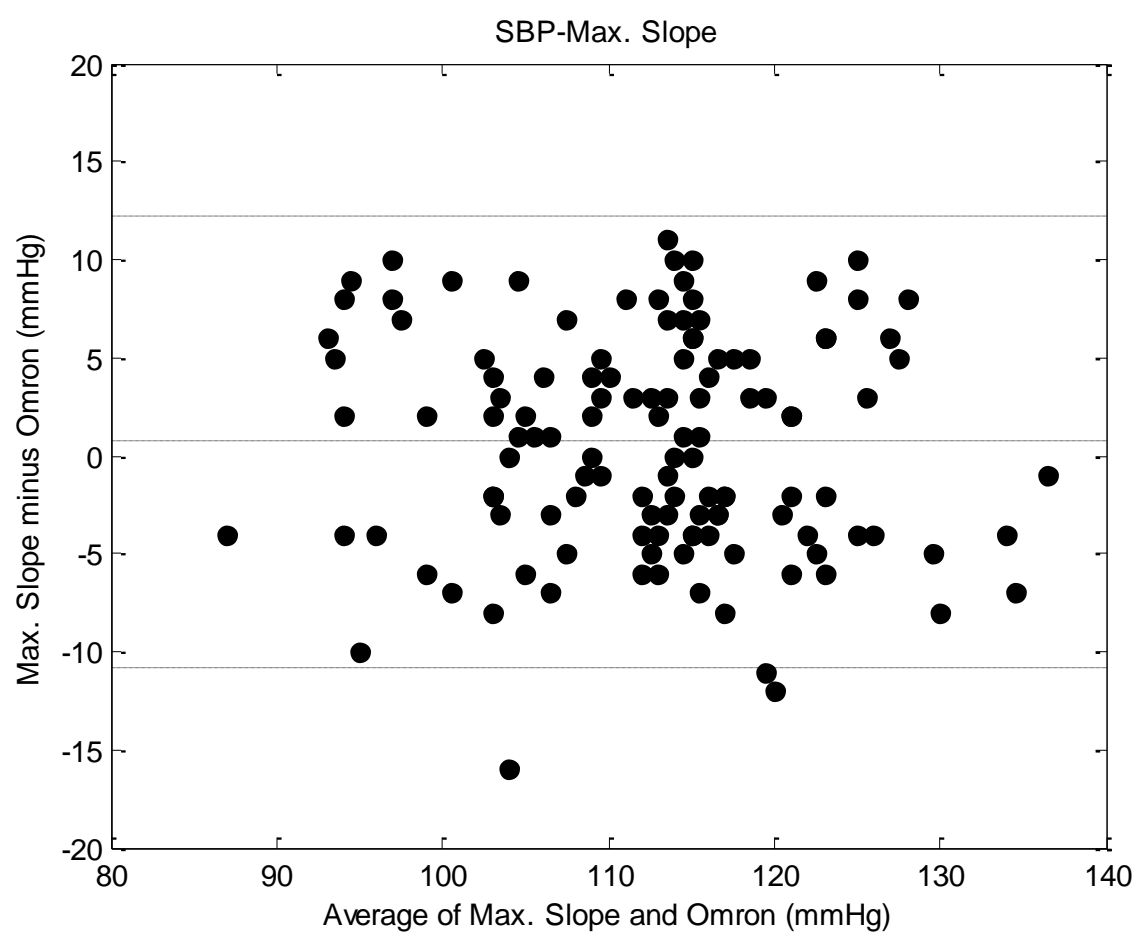

Figure 4.36: Bland Altman plot comparing the Maximum Slope method with OMRON results in SBP 


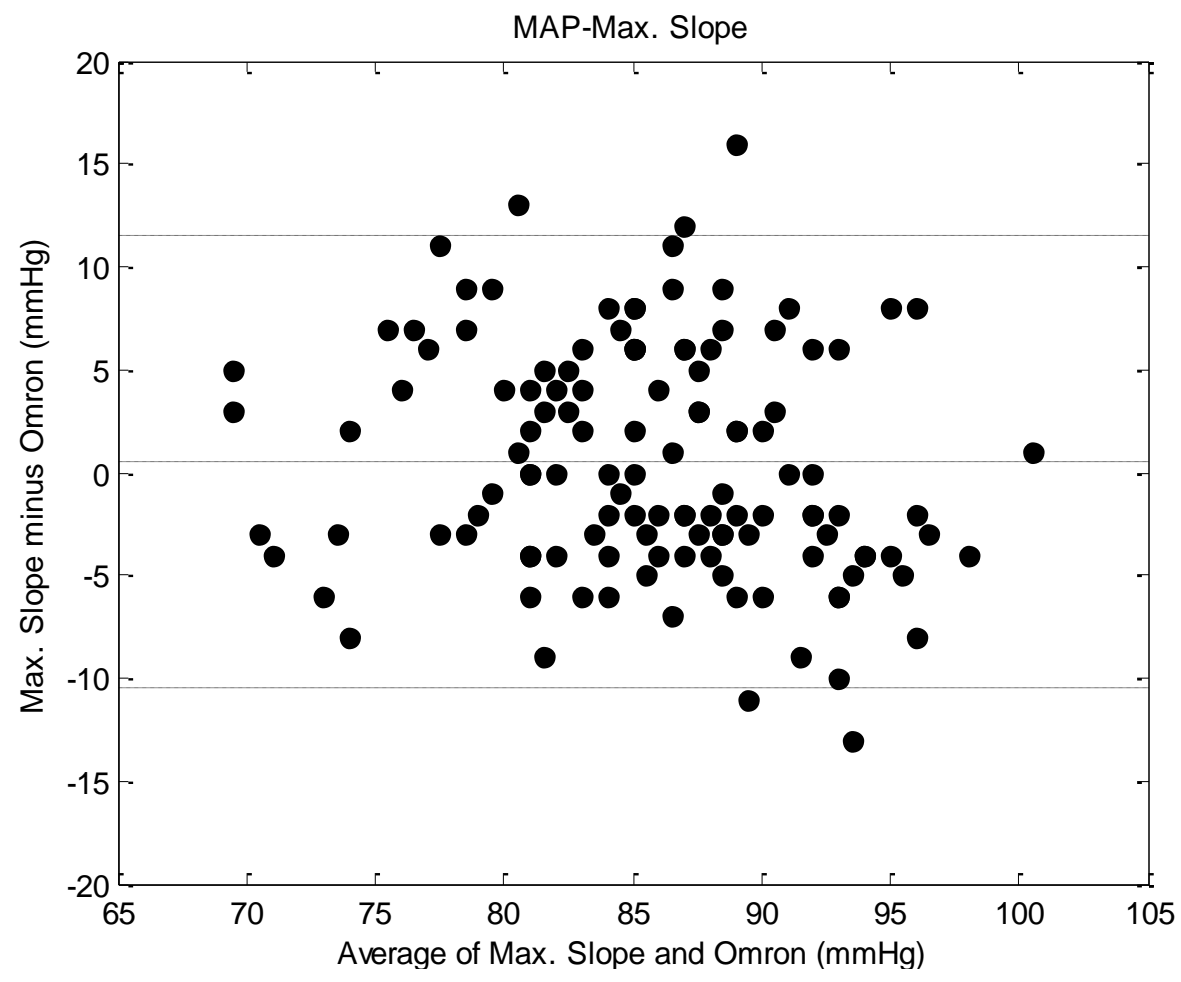

Figure 4.37: Bland Altman plot comparing the Maximum Slope method with OMRON results in MAP

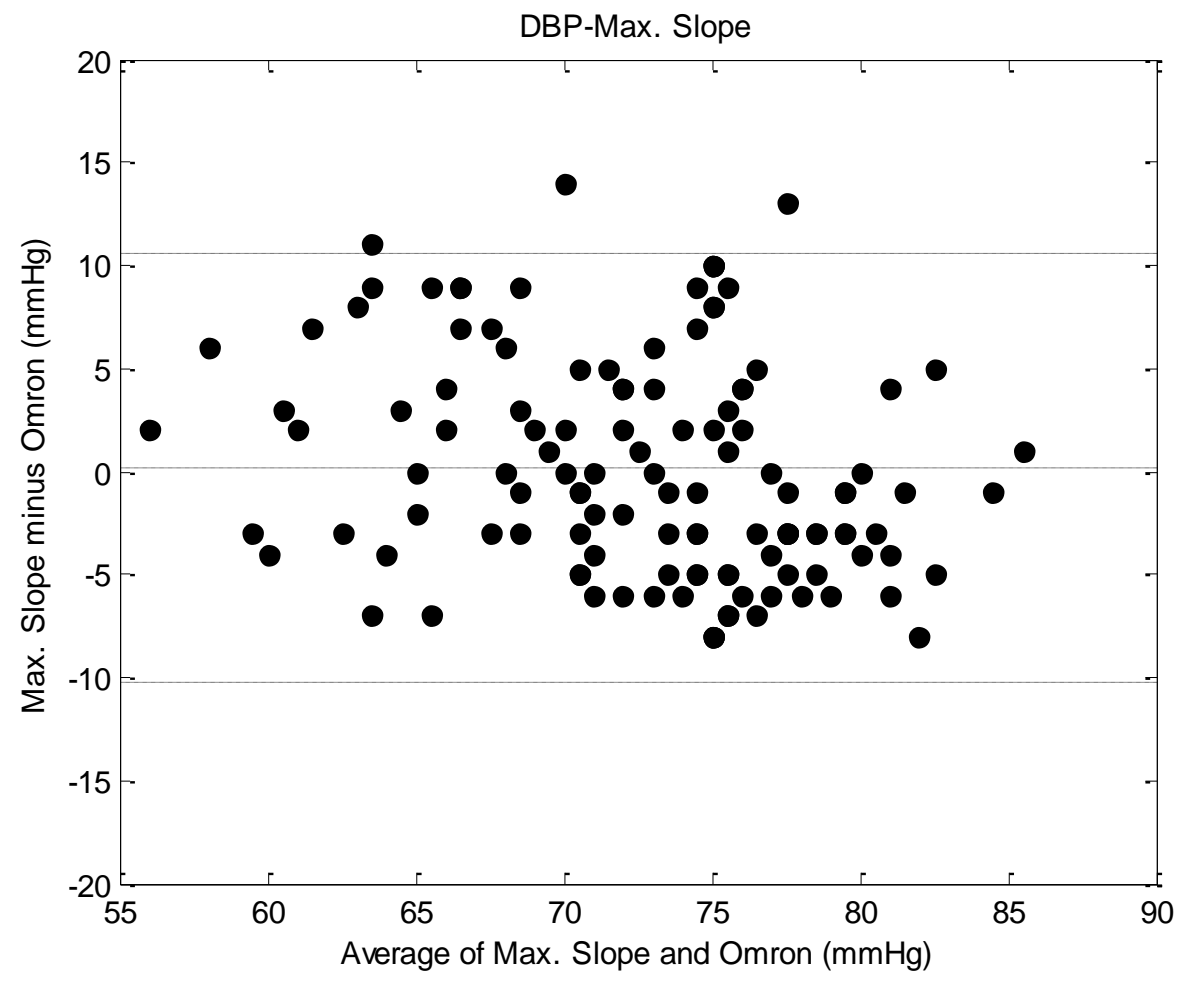

Figure 4.38: Bland Altman plot comparing the Maximum Slope method with OMRON results in DBP 


\subsection{Movement Artifacts}

Similar to other techniques of BP measurement, movements during recording will cause error. Figure 4.39 shows examples of different types of movements in 5 trials of recording with prototype device. Two different kinds of movement were applied during the recordings. First, the subjects were seated with minimum possible movement for a half of the recordings, and then a sudden movement happened in the middle of the recording and subject remained relaxed for rest of the recording. Second, a continuous movement with the arm was applied for the entire recording. Figure 4.40 is an example of pulses with continuous movement during the recording. The BP estimation using presented method, MAA, and OMRON are presented in Table 4.26. Similar to the other projects done in this thesis work, OMRON recordings were taken before the recordings with the prototype device. Subjects remained relaxed during OMRON recordings and no movements were applied and OMRON results were used as reference. Although the proposed method estimated BP for first and fifth trial, but the estimated values were influenced by movement especially at the pressure region that the movement was applied.

Table 4.26: Blood Pressure Estimation with Movement

\begin{tabular}{|c|c|c|c|c|c|c|}
\hline & $\mathrm{AI}$ & $\mathrm{SI}$ & $\mathrm{RI}$ & $\Delta \mathrm{T} / \mathrm{T}$ & MAA & OMRON \\
\hline \multirow{4}{*}{ SBP } & 112 & 112 & 110 & 110 & 119 & 115 \\
\cline { 2 - 7 } & $\mathrm{N} / \mathrm{A}$ & N/A & N/A & N/A & N/A & 111 \\
\cline { 2 - 7 } & $\mathrm{N} / \mathrm{A}$ & N/A & N/A & N/A & N/A & 115 \\
\cline { 2 - 7 } & N/A & N/A & N/A & N/A & N/A & 115 \\
\cline { 2 - 7 } & 107 & 106 & 107 & 104 & 115 & 110 \\
\hline \multirow{5}{*}{ MAP } & 81 & 79 & 79 & 78 & 85 & 89 \\
\cline { 2 - 7 } & N/A & N/A & N/A & N/A & N/A & 90 \\
\cline { 2 - 7 } & N/A & N/A & N/A & N/A & N/A & 88 \\
\cline { 2 - 7 } & N/A & N/A & N/A & N/A & N/A & 88 \\
\cline { 2 - 7 } & 85 & 84 & 85 & 84 & 82 & 87 \\
\hline \multirow{5}{*}{ DBP } & 66 & 67 & 67 & 68 & 71 & 74 \\
\cline { 2 - 7 } & N/A & N/A & N/A & N/A & N/A & 80 \\
\cline { 2 - 7 } & N/A & N/A & N/A & N/A & N/A & 75 \\
\cline { 2 - 7 } & N/A & N/A & N/A & N/A & N/A & 74 \\
\cline { 2 - 7 } & 71 & 71 & 72 & 72 & 69 & 75 \\
\hline
\end{tabular}




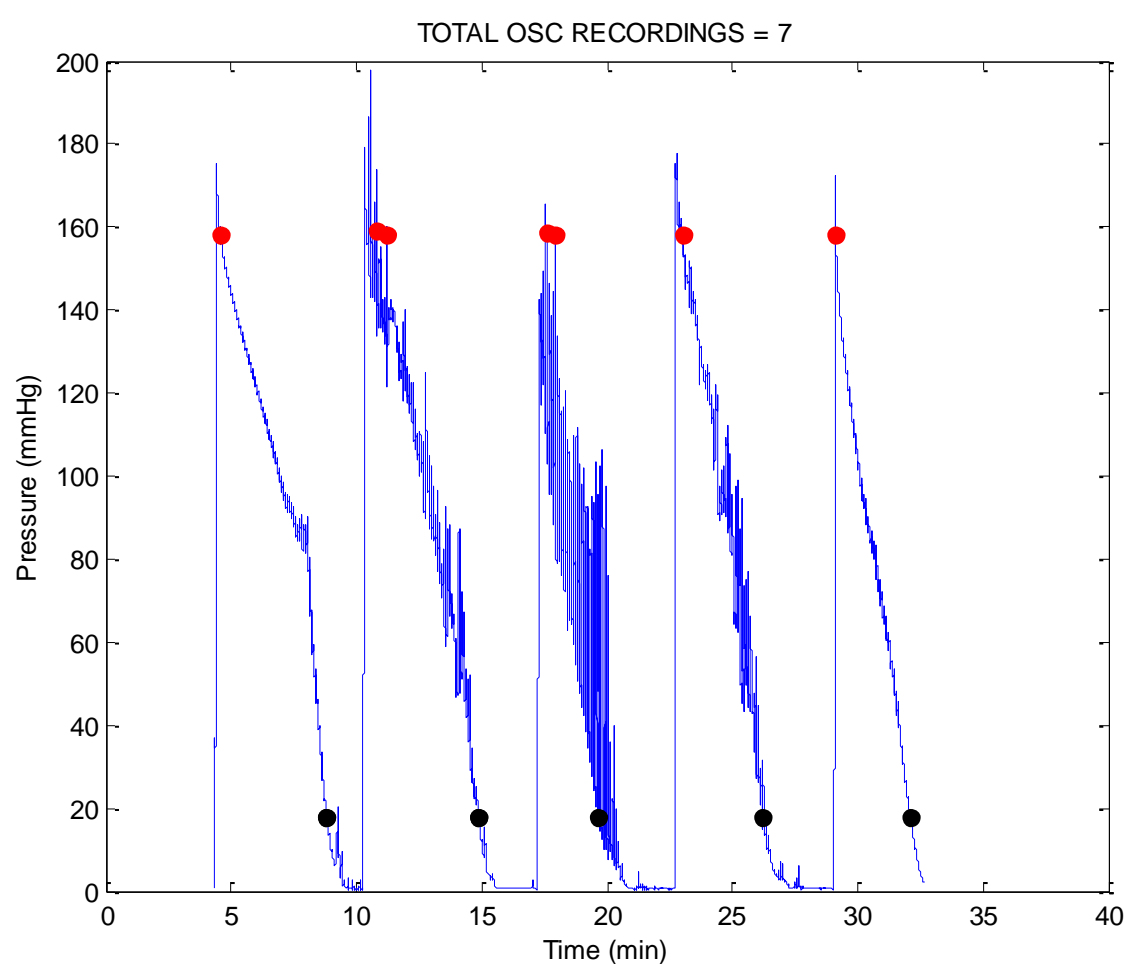

Figure 4.39: Cuff Deflation Pressure Waveforms with Movement during the Recording

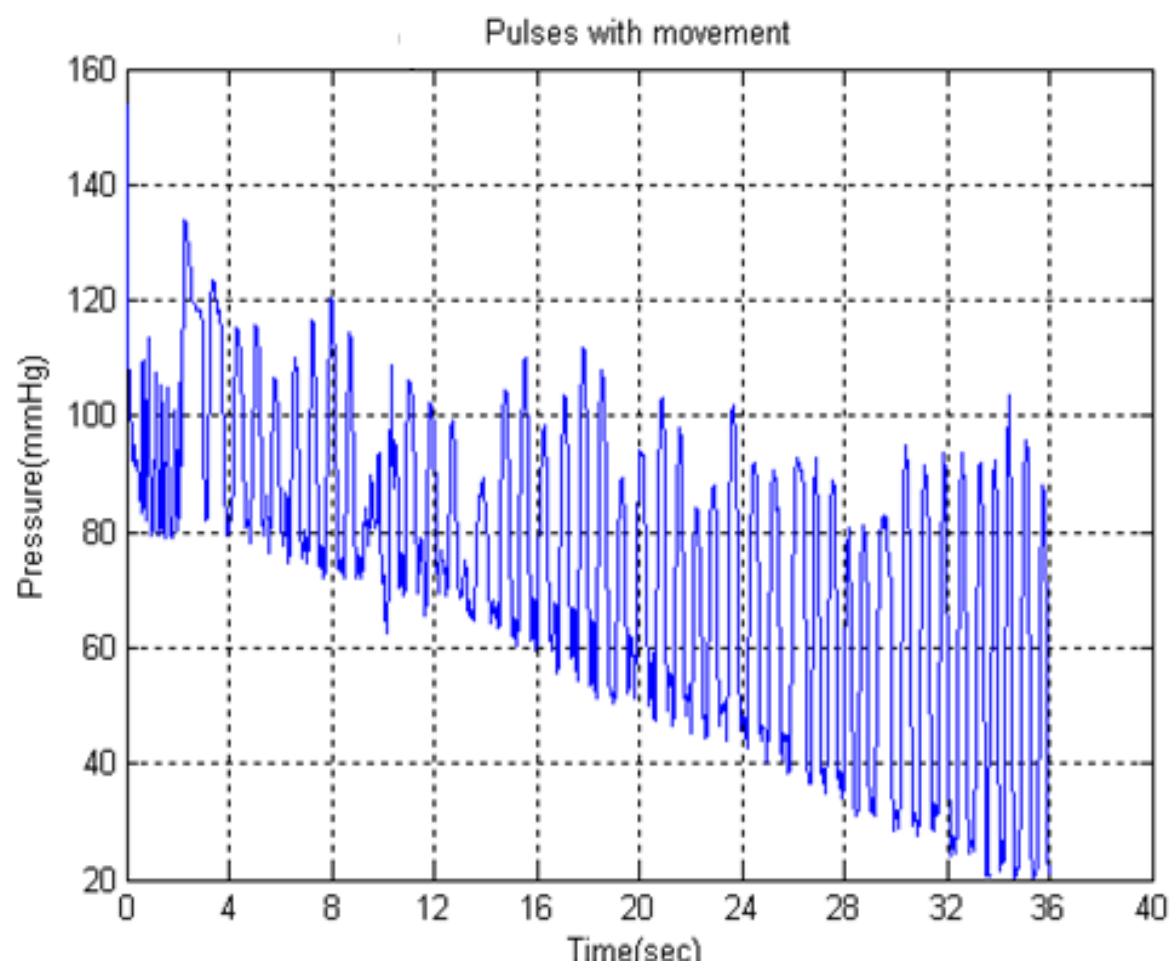

Figure 4.40: Pulses with Movement of Arm during the Recording 


\section{Chapter 5: Discussion}

\subsection{Oscillometric Pulse Morphology}

In the first part of this thesis work, characteristics of the pulse waveforms for different age groups and different pressure points were obtained and compared. We showed that pulses change their characteristics with age. The dicrotic notch on pulses becomes less visible or in some cases even not visible for older subjects. This is caused by reducing the flexibility of the large arteries with aging [14]. Existence of the notch can help to find the location of the second peak of the pulse waveform.

Oscillometric pulse waveform (Oscillometric oscillation) can be detected from the cuff deflation pressure waveform. Pulses at different pressure points showed to have different shape and characteristics. In theory, the Oscillometric maximum amplitude occurs when arterial compliance is maximum or in the other word when the transmural pressure is zero [66]. At MAP, the pressure inside the cuff is equal to the pressure of the artery which would make the transmural pressure zero. Zero transmural pressure means the arterial compliance is maximum and the maximum amplitude occurs. In the first part of the thesis, by computing the amplitudes of the pulse waveforms, it was observed that around MAP region on cuff deflation waveform, pulses have maximum amplitudes.

From our observation, the dicrotic notch was mostly visible around SBP region on cuff deflation pressure waveform and became less visible by going from SBP region to DBP region. Therefore, characteristics and shape of the pulses changed at different pressure points. For every individual the characteristics of the pulses at SBP, MAP, and DBP were computed and the changes toward DBP were observed. 
Reflection Index, Stiffness Index, Crest Time, Relative Crest Time, and Relative Dicrotic Wave Time showed to increase by age while Augmentation Index, $\Delta \mathrm{T} / \mathrm{T}$ ratio, Dicrotic Wave Time, Time Delay Parameter, and area under systolic curve were reducing by age. Also, pulse waveform characteristics varied at different pressure points which can be the result of existence of the dicrotic notch on the waveform, changing of the pressure parameters and time parameters of the pulse waveform.

Healthy subjects at a same age group showed to have similar pulse characteristics at systolic, MAP and diastolic regions. Since the cardiovascular disorders can influence the characteristics of the pulse waveforms, they can be predicted using pulse morphology.

\subsection{Blood Pressure Estimation Using Pulse Morphology}

In Oscillometric method, MAP is estimated by using the Oscillometric pulse with maximum amplitude. The maximum amplitude at MAP is due to the zero transmural pressure [66]. Since the amplitude of the pulse at MAP is more than other pulses around it, an obvious sharp peak will create by plotting the characteristics of the pulses from cuff deflation waveform (Figure 4.2 to Figure 4.5). MAP pulse divides the signal into systolic and diastolic regions. Systolic region is on the left side of MAP while diastolic region is on the right side.

Plotting four parameters (Reflection Index, Augmentation Index, Stiffness Index, and $\Delta T / T$ ratio) of every pulse from cuff deflation pressure waveform provide two different types of signals to estimate blood pressure which they had opposite characteristics of each other. Reflection Index and Stiffness Index showed to have similar graphs. MAP tends to be the maximum point in Reflection Index and Stiffness Index graphs. The systolic region is convex 
and the diastolic region is concave. Minimum of the convex graph represents systolic pulse and maximum of the diastolic region represents diastolic pulse.

Augmentation Index and $\Delta \mathrm{T} / \mathrm{T}$ ratio had opposite shape of other type. Minimum point tends to represent MAP pulse. Systolic region is concave and diastolic region is convex. The maximum of systolic region represents systolic pulse and the minimum of the diastolic region represents diastolic pulse.

Nine pulses at minimum at maximum points of systolic and diastolic regions were chosen and the mean of the pressures of the chosen pulses defined SBP and DBP. Due to the possible small movements of the limbs during the recording, the shape and characteristics of the pulse waveforms could be influenced. Therefore, nine pulses at each region were chosen to estimate BP. Comparing the proposed method of BP estimation and MAA with OMRON results in Chapter 4, Section 4.3, we observed that the proposed method had smaller error at SBP and MAP and MAA had smaller error at DBP with OMRON as reference.

\subsection{SBP and DBP Estimation using Curve Fitting Technique}

The SBP and DBP estimated using curve fitting technique was compared with the SBP and DBP computed from the simple method of averaging nine minimum and maximum points of systolic and diastolic regions of plots of parameters.

Comparing Figure 4.7 and Figure 4.9, Bland Altman plots of comparing the SBP and DBP estimated from the nine points with OMRON with Figure 5.1 and Figure 5.2, we can observe that results estimated with simple method of averaging nine pulses provided more similarity with OMRON recordings. In Figure 4.7 and Figure 4.9, the final results are more concentrated to the center than Figure 5.1 and Figure 5.2. Although, both techniques were tested on 18 subjects only 
and more subjects are needed for choosing the better technique of estimating SBP and DBP in the presented method. Also both techniques are comparing with OMRON recordings, while OMRON is not the gold standard of BP measurement.

\subsection{Respiration in Blood Pressure}

As mentioned in Section2.3, breathing influence the amplitude and frequency modulation of the pulse waveform. Since MAA is dependent on the amplitude of the pulse waveform, this technique of blood pressure estimation can be affected with fast and deep breathing. Examples of BP estimation using MAA with fast and deep breathing are shown in Figure 5.1 and Figure 5.2. The horizontal lines in both figures represent the OMRON results which were taken before the recording with prototype device. The points with the highest amplitude represent MAP estimated by MAA. Points on the left side of MAP represent SBP and points on the right side of MAP represent DBP of the subjects.

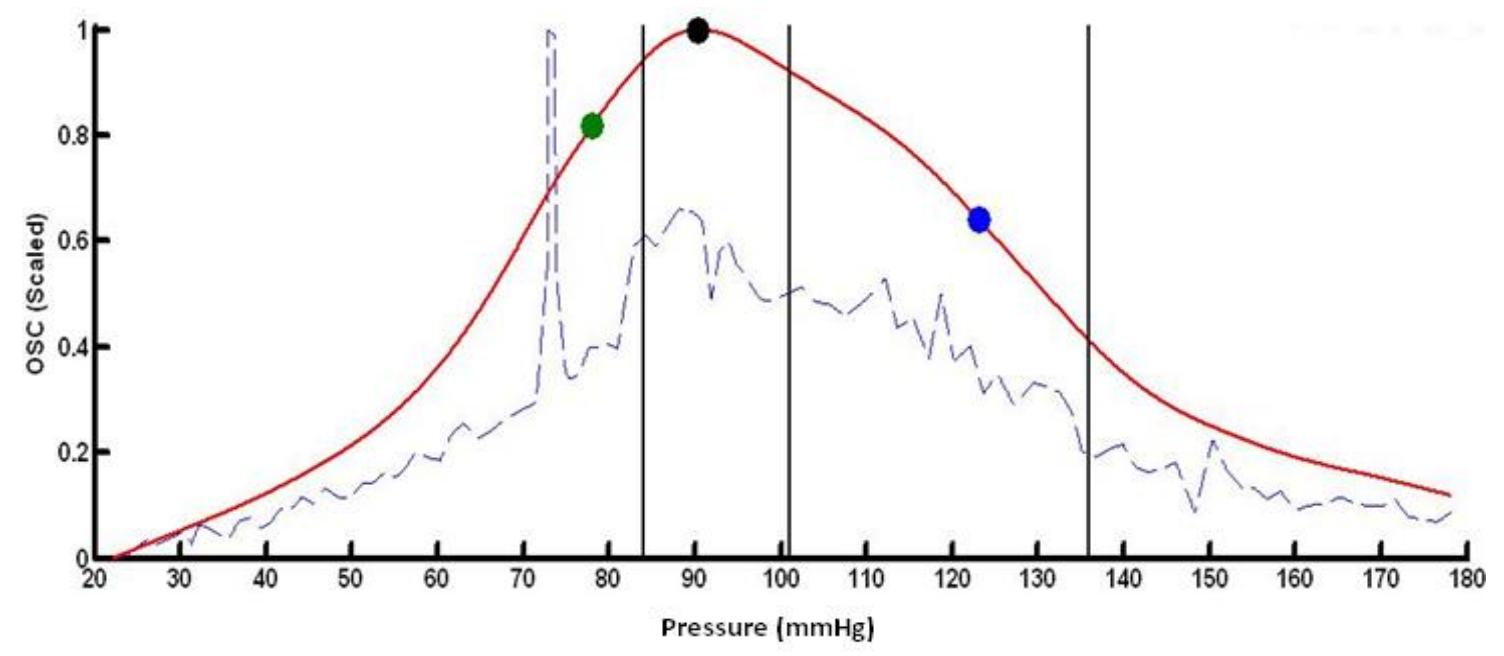

Figure 5.1: Blood Pressure Estimation using MAA with Fast Breathing 


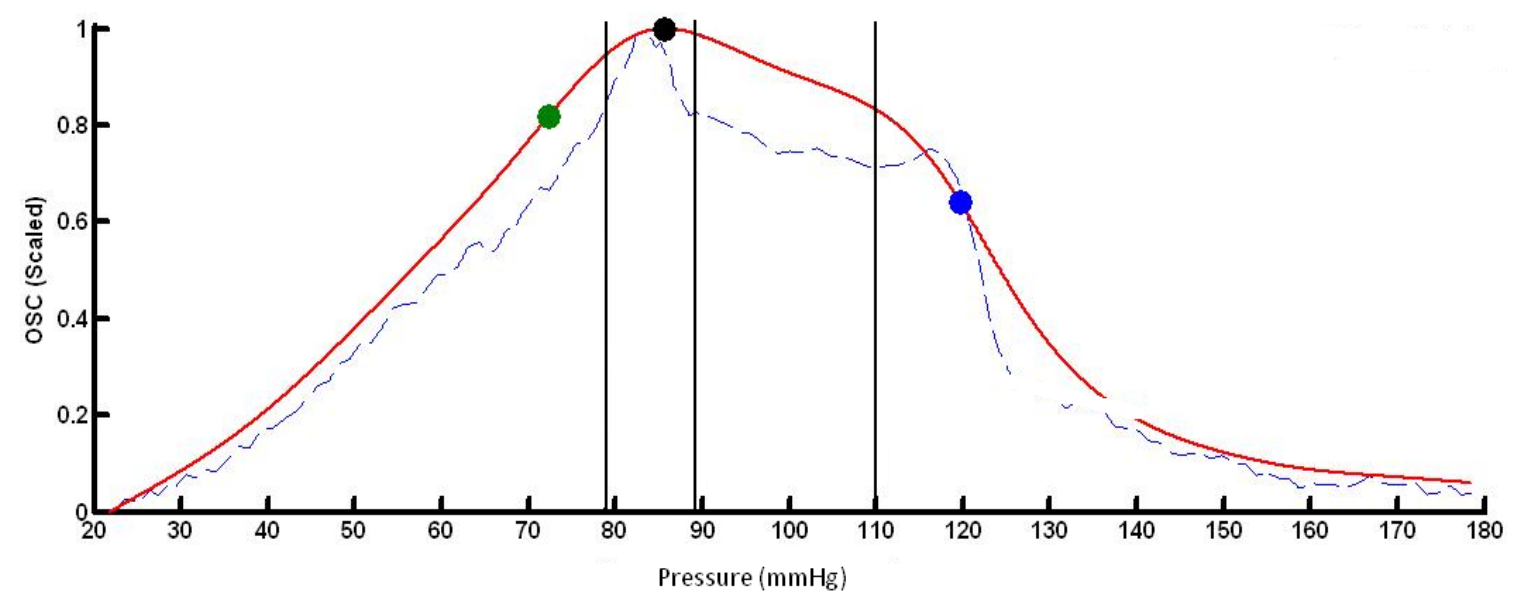

Figure 5.2: Blood Pressure Estimation using MAA with Deep Breathing

The BP estimation with the presented method using the parameters of the pulse waveform is dependent on the changes of the characteristics of the pulse waveforms from systolic region toward the diastolic region. Although, the characteristics of the pulse waveforms changes from normal breathing to either fast or deep breathing, but the changes of the characteristics from higher pressure points to lower pressure point showed to be same in normal, fast, and deep breathing. Figure 5.3 shows the BP estimation using four parameters of pulse waveforms from cuff deflation pressure waveform with fast breathing and Figure 5.4 shows the BP estimation using the presented method with deep breathing.

As described in Section 4.3, in plots of Augmentation Index and $\Delta \mathrm{T} / \mathrm{T}$ ratio minimum point represents the MAP pulse; the left side of the MAP is systolic region and is concave and the maximum of the systolic region represents systolic pulse. The right side of the MAP is diastolic region and is convex and the minimum of the diastolic region represents diastolic pulse. The plots of Stiffness Index and Reflection Index had opposite characteristics. The maximum point represents the MAP pulse. Systolic region is convex and minimum of the systolic region shows systolic pulse. Diastolic region is concave and maximum of the diastolic region represents diastolic pulse. 
The comparison of blood pressure estimation using proposed method with MAA and OMRON results in table 4.23 and 4.24 showed that the presented method has closer estimation to OMRON results than MAA. It should be also noticed that only six recordings with breathing were taken and more recordings are needed for more accurate conclusion. Again, the presented method should also be compared to the gold standard of blood pressure estimation or more accurate methods of blood pressure measurements than OMRON device.

\subsection{Blood Pressure Estimation using Maximum Slope}

The location of the maximum slope of each pulse waveform was obtained from the first derivative of the pulses. The peak with maximum amplitude from the first derivative signal was mapped to the original pulse to detect the location of the maximum slope. Plotting the maximum slope of the pulses from cuff deflation pressure waveform provided an envelope. Similar to MAA techniques, the maximum point on the envelope is used to determine MAP. Systolic and diastolic points can be obtained by their own ratios from MAP at their corresponding regions.

The advantage of estimating blood pressure using maximum slope over MAA is that this technique may be more robust than MAA [78]. In MAA, BP is estimated based on the amplitude of the first peak of the pulse waveforms and therefore, noise can either affect the amplitude of pulses or create extra peaks which would make the BP estimation less accurate.

Results of student's t test in Table 2.25 showed that $\mathrm{P}$ values for the BP estimation using maximum slope are lower than $\mathrm{P}$ values for BP estimation using MAA, which supports the use of maximum slope of the pulse waveforms for BP estimation. 

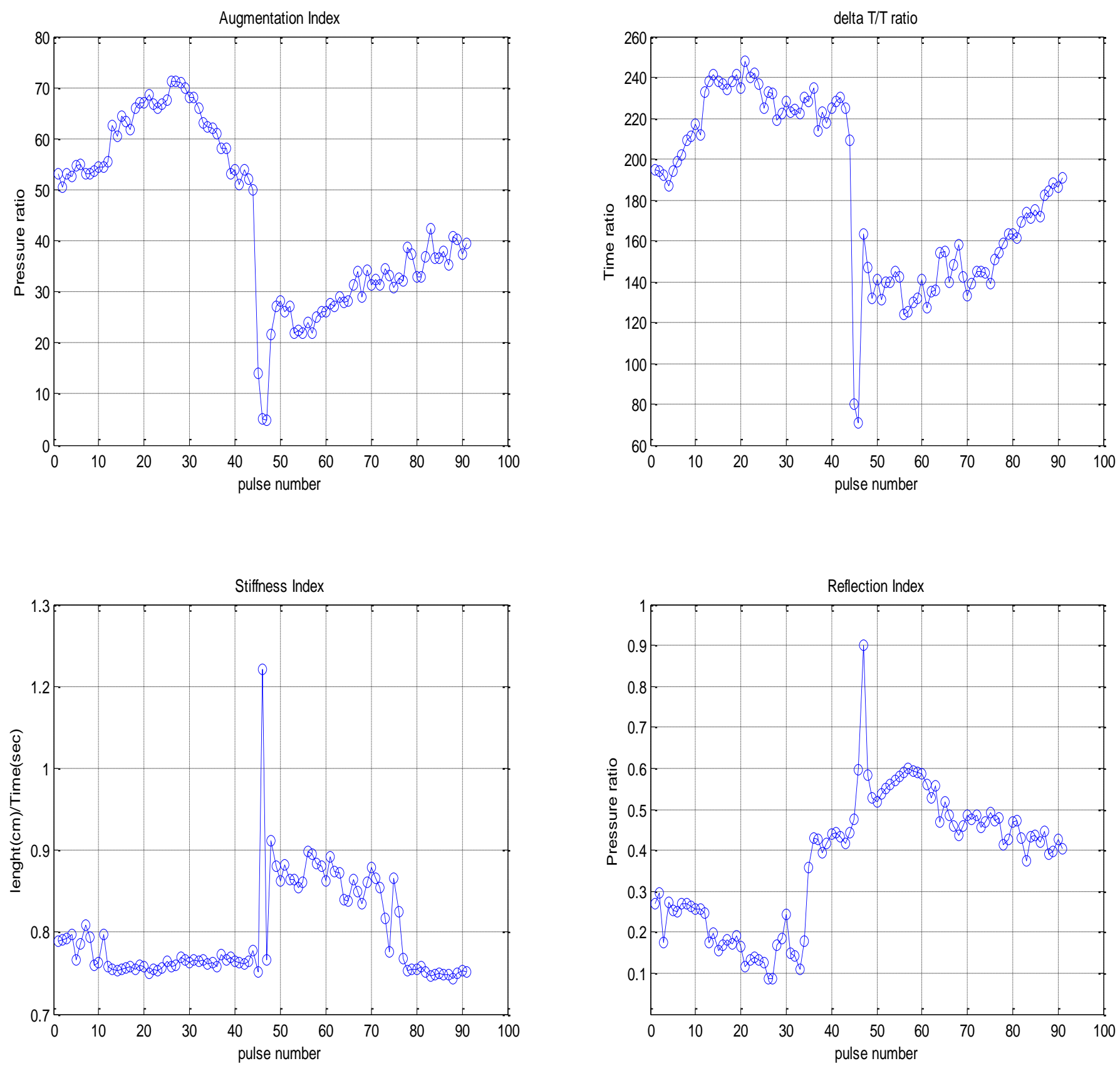

Figure 5.3: Blood Pressure Estimation using four parameters of Pulse Waveforms with Fast Breathing 

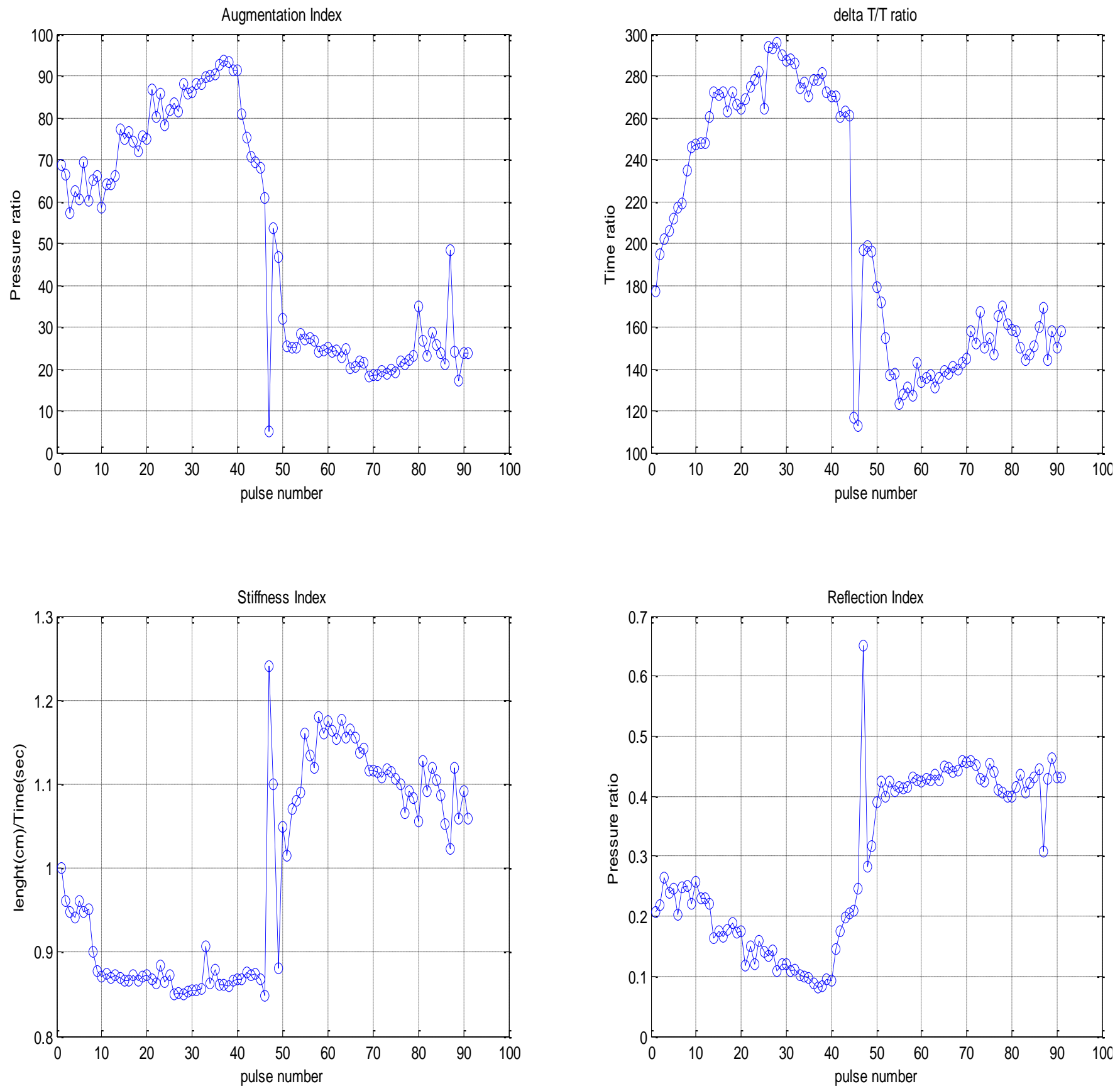

Figure 5.4: Blood Pressure Estimation using four parameters of Pulse Waveforms with Deep

\section{Breathing}




\subsection{Movement Artifacts}

Similar to the other methods of blood pressure estimation, the presented method will fail in certain conditions and BP cannot be estimated. Unlike other methods of BP estimation, recordings should be taken slower than usual for better detection of second peak of the blood pressure pulse waveform. Other methods such as MAA focus on the amplitude of the first peak (systolic peak) of the pulse waveform while in this method the amplitude of both peaks from pulse waveform are needed to be studied for BP estimation. Fast deflation of the cuff during the recording would affect the shape of the pulses specially the second peak detection. Since the systolic peak of the pulse waveform has the highest amplitude, detecting the first peaks of the pulse waveforms has no issue, but the second peak detection of the pulse waveform will be difficult especially in the pressure ranges that the dicrotic notch is less visible or in some cases not visible.

Similar to other techniques of BP measurement, movements during recording will cause error. Figure 5.7 shows examples of different types of movements in 5 trials of recording with prototype device. Two different kinds of movement were applied during the recordings. First, the subjects were seated with minimum possible movement for a half of the recordings, and then a sudden movement happened in the middle of the recording and subject remained relaxed for rest of the recording. Second, a continuous movement with the arm was applied for the entire recording. In the second case of movement with continuous movement of the arm, both MAA and the proposed method failed to estimate SBP, MAP, and DBP since the noise caused by the movement affected the location, shape and characteristics of all the pulses from cuff deflation pressure waveform. In Figure 5.7, first and fifth trials have the first type of movement when a sudden movement applied in the middle of the recording. Second, third, and forth trials are 
examples of recordings with continuous movement of the arm for the entire recording. In the case of second type of movement, pulse waveforms are extremely influenced by movement artifacts. Figure 5.8 is an example of pulses with continuous movement during the recording.

\subsection{Robustness of the Algorithm}

The proposed method was tested on 18 healthy subjects, with 175 recordings over all. Some of the computed results using the presented method were out of the acceptable range of comparing with OMRON recordings. In SBP estimation using parameters of the blood pressure pulse waveforms, 12 recordings using AI, 14 recordings using $\Delta \mathrm{T} / \mathrm{T}$ ratio, 17 recordings using SI, and 17 recordings using RI were out of $\pm 10 \mathrm{mmHg}$ range. In MAP estimation, 1 recording for AI, $\Delta \mathrm{T} / \mathrm{T}$ ratio, and SI individually and 2 recordings for RI were out of $\pm 10 \mathrm{mmHg}$ range. In DBP estimation, 12 recordings in AI, 14 recordings in $\Delta \mathrm{T} / \mathrm{T}$ ratio, 9 recordings in SI, and 11 recordings in RI were out of the $\pm 10 \mathrm{mmHg}$ range. In comparison of the proposed method of estimating BP using pulse parameters with OMRON recordings, MAP had the most accurate results. Also Bland Altman plots of MAP showed that MAP estimation using proposed algorithm is closer to OMRON in comparison with SBP and DBP estimation.

In BP estimation using the maximum slope on the pulse waveform, 10 recordings at DBP, 10 recordings at MAP, and 12 recordings at $\mathrm{SBP}$ were out of $\pm 10 \mathrm{mmHg}$ range. As mentioned before. The BP estimation using the maximum slope on the pulse waveform is less sensitive to noise but it may be compared to other methods of blood pressure measurements as well. 


\section{Chapter 6: Conclusion and Future works}

\subsection{Summary of Thesis}

Blood Pressure (BP) is a vital sign and provides significant physiological information about patients' health. It fluctuates over time and many factors can affect systolic blood pressure (SBP) and diastolic blood pressure (DBP). Oscillometric pulse waveforms can be recorded during the Oscillometric recording and pulse morphology can be used to detect various cardiovascular diseases. The presented work is intended to analyze the characteristics of the Oscillometric pulse waveforms at different pressure range and develop a new technique of estimating BP based on pulse morphology.

Characteristics of the pulse waveforms at systolic, MAP and diastolic regions were computed for all the participants. Pulse waveform characteristics were compared at different pressure points for every subject. Also, subjects were divided into three different age groups and the parameters were compared for different age groups at different pressure ranges. Oscillometric pulse waveforms were detected from cuff deflation pressure waveform. This study evaluated the characteristics of all the pulses from cuff deflation waveform and the plots of those parameters used for the BP estimation. As part of presented work, the maximum slope of the pulse waveforms were used to estimate BP. The location of maximum slopes was determined from the first derivative of the pulse waveform and was mapped to the original pulse waveform. The amplitudes of the locations of the maximum slopes of all the pulse waveform were plotted and an envelope was detected for all the recordings. Similar to MAA method the maximum amplitude 
of the envelope represented MAP pulse and corresponding systolic and diastolic pulses were determined from MAP pulse with their ratios respectively.

The effects of movement and breathing artifacts were observed on the proposed method and MAA. The proposed method showed to be more robust in cases of breathing artifacts than MAA.

The proposed technique was testing using a prototype device. A cuff was placed around the left arm and inflated above the expected SBP and deflated slowly below the expected DBP. The cuff pressure was recorded using a Vernier pressure Transducer. OMRON HEM-790 ITCAN was placed around the right arm and OMRON recording was taking before the prototype device and its results were saved to use as reference to compare the results of proposed method with.

Blood pressure estimation using pulse morphology was proposed in this thesis work. The incorporation of this method can improve the capabilities of the existing Oscillometric devices and can help improving accuracy of the BP estimation in current practice and methods.

\subsection{Contributions}

The main contributes of this thesis work are as the follow:

- Analyzing the characteristics of the pulse waveforms at different pressure points for every individual and comparing the characteristics for different age groups

- Developing an new method of blood pressure estimation using parameters of the all the pulses detected from the cuff deflation pressure waveform

- Developing a new method of estimating blood pressure using the maximum slope of the pulse waveforms determined from the first derivative of each pulse waveform. 
- Initial analysis of the robustness of the proposed method under breathing and movement artifacts and comparison with MAA and BP values estimated with OMRON device

\subsection{Future Work}

The presented technique of estimating blood pressure had various limitations that may be addressed as future work. The major limitation that the presented methods are facing was validation. The results of presented methods were checked and compared with the results of OMRON blood pressure measurement device and MAA method. However, invasive method of blood pressure measurement (intra-arterial) is considered to be a gold standard of blood pressure and comparing the results with this method would lead to more definite validation. Also, in noninvasive methods Auscultatory method is the standard and would allow for better validation. For future work the results of the presented method may be compared with nurse measurements using invasive or Auscultatory method.

The proposed methodology was tested on 18 subjects only. Testing the method on a larger population can provide more evidence of accuracy of this method. Also, none of the participants had a history of any cardiovascular diseases. Since the blood pressure estimation in the proposed method is based on the characteristics of the pulse, some of cardiovascular disease can affect the shape and characteristics of the pulse by creating extra peaks or changing the time parameters and pressure parameters of the pulse waveform. Therefore, the proposed method is needed to be tested on more people and with subjects with different health conditions.

Medications that use for high blood pressure have different effect on different organs of the body. Some of them influence contraction and relaxation of heart muscle which would change the shape and characteristics of the pulse waveform and the presented method of blood pressure 
estimation using pulse morphology will not work in this case. However, the new method of blood pressure estimation is needed to be checked on subjects that take other kinds of medications.

The method presented uses 4 parameters of the pulse waveform. Each parameter was separately examined to estimate BP. Future work may consist of combining the parameters of the pulse waveform for more accurate BP estimation based on the advantages of either pressure or time characteristics of the pulses.

Since the plots of parameters shows the characteristics of all the pulses from cuff deflation waveform, other possible information can be detected from those graphs. Since pulse morphology is usually used for prediction of cardiovascular disorders the plots of parameters can be used for cardiovascular system check and blood pressure estimation at the same time. 


\section{APPENDIX A- BP Estimated by 4 Parameters of Pulse Waveform}

\begin{tabular}{|l|c|c|c|c|c|c|}
\hline $\begin{array}{l}\text { Subject 1 } \\
\text { SBP }\end{array}$ & AI & $\Delta \mathrm{T} / \mathrm{T}$ & SI & RI & MAA & Omron \\
\hline Trial 1 & 110 & 109 & 129 & 130 & 120 & 121 \\
\hline Trial 2 & 130 & 135 & 118 & 118 & 121 & 121 \\
\hline Trial 3 & 128 & 128 & 126 & 126 & 117 & 126 \\
\hline Trial 4 & 126 & 124 & 130 & 128 & 111 & 126 \\
\hline Trial 5 & 109 & 111 & 117 & 116 & 130 & 122 \\
\hline Trial 6 & 115 & 115 & 127 & 124 & 125 & 127 \\
\hline Trial 7 & 118 & 119 & 129 & 128 & 123 & 127 \\
\hline Trial 8 & 124 & 121 & 128 & 128 & 124 & 130 \\
\hline Trial 9 & 125 & 125 & 130 & 131 & 123 & 130 \\
\hline Trial 10 & 138 & 130 & 135 & 136 & 128 & 132 \\
\hline Trial 11 & 124 & 126 & 109 & 108 & 125 & 114 \\
\hline Trial 12 & 113 & 113 & 113 & 115 & 123 & 113 \\
\hline Trial 13 & 112 & 114 & 121 & 126 & 118 & 115 \\
\hline Trial 14 & 126 & 126 & 116 & 120 & 118 & 122 \\
\hline Trial 15 & 122 & 120 & 122 & 124 & 114 & 120 \\
\hline
\end{tabular}

\begin{tabular}{|l|c|c|c|c|c|c|}
\hline $\begin{array}{l}\text { Subject 1 } \\
\text { MAP }\end{array}$ & AI & $\Delta \mathrm{T} / \mathrm{T}$ & SI & RI & MAA & Omron \\
\hline Trial 1 & 88 & 88 & 86 & 86 & 87 & 89 \\
\hline Trial 2 & 87 & 86 & 85 & 84 & 82 & 90 \\
\hline Trial 32 & 90 & 90 & 89 & 89 & 80 & 91 \\
\hline Trial 4 & 92 & 93 & 91 & 91 & 82 & 91 \\
\hline Trial 5 & 84 & 83 & 94 & 95 & 103 & 88 \\
\hline Trial 6 & 90 & 90 & 95 & 95 & 94 & 92 \\
\hline Trial 7 & 86 & 84 & 88 & 88 & 82 & 90 \\
\hline Trial 8 & 91 & 96 & 96 & 94 & 94 & 95 \\
\hline Trial 9 & 95 & 96 & 104 & 103 & 89 & 100 \\
\hline Trial 10 & 90 & 89 & 96 & 91 & 86 & 94 \\
\hline Trial 11 & 78 & 77 & 85 & 86 & 91 & 84 \\
\hline Trial 12 & 80 & 80 & 86 & 86 & 94 & 84 \\
\hline Trial 13 & 82 & 83 & 88 & 90 & 88 & 83 \\
\hline Trial 14 & 87 & 87 & 88 & 84 & 88 & 87 \\
\hline Trial 15 & 86 & 81 & 86 & 81 & 88 & 87 \\
\hline
\end{tabular}

\begin{tabular}{|l|c|c|c|c|c|c|}
\hline $\begin{array}{l}\text { Subject 1 } \\
\text { DBP }\end{array}$ & AI & $\Delta \mathrm{T} / \mathrm{T}$ & SI & RI & MAA & Omron \\
\hline Trial 1 & 63 & 63 & 69 & 68 & 63 & 73 \\
\hline Trial 2 & 62 & 62 & 72 & 71 & 66 & 74 \\
\hline Trial 3 & 74 & 75 & 71 & 70 & 66 & 74 \\
\hline Trial 4 & 81 & 81 & 79 & 81 & 66 & 74 \\
\hline Trial 5 & 81 & 83 & 73 & 76 & 68 & 71 \\
\hline Trial 6 & 79 & 78 & 77 & 75 & 69 & 75 \\
\hline Trial 7 & 78 & 78 & 72 & 76 & 67 & 71 \\
\hline Trial 8 & 71 & 72 & 76 & 78 & 70 & 78 \\
\hline Trial 9 & 80 & 83 & 80 & 83 & 73 & 85 \\
\hline Trial 10 & 76 & 78 & 81 & 80 & 69 & 75 \\
\hline Trial 11 & 69 & 70 & 67 & 69 & 71 & 69 \\
\hline Trial 12 & 65 & 69 & 67 & 68 & 74 & 69 \\
\hline Trial 13 & 65 & 69 & 68 & 67 & 69 & 67 \\
\hline Trial 14 & 70 & 73 & 77 & 80 & 74 & 70 \\
\hline Trial 15 & 70 & 71 & 77 & 79 & 73 & 70 \\
\hline
\end{tabular}

\begin{tabular}{|l|c|c|c|c|c|c|}
\hline $\begin{array}{l}\text { Subject 2 } \\
\text { SBP }\end{array}$ & AI & $\Delta \mathrm{T} / \mathrm{T}$ & SI & RI & MAA & Omron \\
\hline Trial 1 & 128 & 126 & 133 & 133 & 123 & 136 \\
\hline Trial 2 & 124 & 123 & 126 & 125 & 122 & 129 \\
\hline Trial 3 & 136 & 136 & 130 & 136 & 119 & 130 \\
\hline Trial 4 & 129 & 131 & 133 & 131 & 120 & 119 \\
\hline Trial 5 & 130 & 130 & 130 & 131 & 121 & 132 \\
\hline Trial 6 & 128 & 127 & 131 & 133 & 127 & 131 \\
\hline Trial 7 & 127 & 129 & 130 & 128 & 124 & 127 \\
\hline Trial 8 & 116 & 116 & 113 & 115 & 123 & 120 \\
\hline Trial 9 & 126 & 125 & 128 & 128 & 121 & 119 \\
\hline Trial 10 & 124 & 126 & 126 & 126 & 122 & 126 \\
\hline Trial 11 & 118 & 118 & 107 & 109 & 114 & 116 \\
\hline Trial 12 & 119 & 117 & 122 & 121 & 116 & 117 \\
\hline Trial 13 & 120 & 122 & 115 & 118 & 115 & 115 \\
\hline Trial 14 & 110 & 110 & 112 & 114 & 112 & 114 \\
\hline Trial 15 & 108 & 106 & 109 & 109 & 115 & 113 \\
\hline
\end{tabular}

\begin{tabular}{|l|c|c|c|c|c|c|}
\hline $\begin{array}{l}\text { Subject 2 } \\
\text { MAP }\end{array}$ & AI & $\Delta \mathrm{T} / \mathrm{T}$ & SI & RI & MAA & Omron \\
\hline Trial 1 & 98 & 98 & 102 & 102 & 90 & 101 \\
\hline Trial 2 & 94 & 94 & 96 & 96 & 92 & 96 \\
\hline Trial 3 & 94 & 94 & 97 & 97 & 91 & 95 \\
\hline Trial 4 & 90 & 90 & 91 & 91 & 88 & 92 \\
\hline Trial 5 & 94 & 93 & 96 & 96 & 95 & 95 \\
\hline Trial 6 & 94 & 94 & 95 & 94 & 100 & 97 \\
\hline Trial 7 & 96 & 98 & 99 & 99 & 97 & 95 \\
\hline Trial 8 & 101 & 101 & 100 & 105 & 97 & 91 \\
\hline Trial 9 & 92 & 90 & 91 & 91 & 92 & 92 \\
\hline Trial 10 & 91 & 91 & 93 & 93 & 96 & 99 \\
\hline Trial 11 & 80 & 79 & 79 & 80 & 80 & 86 \\
\hline Trial 12 & 90 & 90 & 89 & 89 & 88 & 91 \\
\hline Trial 13 & 84 & 84 & 86 & 86 & 87 & 88 \\
\hline Trial 14 & 86 & 86 & 87 & 87 & 86 & 87 \\
\hline Trial 15 & 84 & 85 & 86 & 86 & 87 & 85 \\
\hline
\end{tabular}

\begin{tabular}{|l|c|c|c|c|c|c|}
\hline $\begin{array}{l}\text { Subject 2 } \\
\text { DBP }\end{array}$ & AI & $\Delta \mathrm{T} / \mathrm{T}$ & $\mathrm{SI}$ & $\mathrm{RI}$ & MAA & Omron \\
\hline Trial 1 & 80 & 82 & 80 & 81 & 78 & 84 \\
\hline Trial 2 & 75 & 76 & 72 & 74 & 80 & 80 \\
\hline Trial 3 & 76 & 77 & 70 & 75 & 78 & 77 \\
\hline Trial 4 & 76 & 77 & 71 & 73 & 76 & 79 \\
\hline Trial 5 & 80 & 84 & 83 & 83 & 81 & 76 \\
\hline Trial 6 & 90 & 86 & 87 & 87 & 85 & 80 \\
\hline Trial 7 & 75 & 79 & 83 & 82 & 86 & 79 \\
\hline Trial 8 & 75 & 73 & 72 & 74 & 84 & 76 \\
\hline Trial 9 & 83 & 80 & 76 & 75 & 80 & 79 \\
\hline Trial 10 & 89 & 86 & 79 & 76 & 83 & 86 \\
\hline Trial 11 & 65 & 68 & 61 & 64 & 69 & 71 \\
\hline Trial 12 & 77 & 72 & 79 & 82 & 74 & 78 \\
\hline Trial 13 & 75 & 74 & 77 & 79 & 74 & 75 \\
\hline Trial 14 & 76 & 74 & 81 & 83 & 75 & 74 \\
\hline Trial 15 & 70 & 68 & 64 & 66 & 76 & 71 \\
\hline
\end{tabular}




\begin{tabular}{|l|c|c|c|c|c|c|}
\hline $\begin{array}{l}\text { Subject 3 } \\
\text { SBP }\end{array}$ & AI & $\Delta \mathrm{T} / \mathrm{T}$ & $\mathrm{SI}$ & $\mathrm{RI}$ & MAA & Omron \\
\hline Trial 1 & 108 & 106 & 110 & 111 & 107 & 105 \\
\hline Trial 2 & 96 & 98 & 94 & 92 & 108 & 100 \\
\hline Trial 3 & 106 & 106 & 112 & 107 & 114 & 98 \\
\hline Trial 4 & 96 & 96 & 99 & 97 & 106 & 96 \\
\hline Trial 5 & 104 & 103 & 101 & 100 & 112 & 110 \\
\hline Trial 6 & 109 & 109 & 111 & 112 & 104 & 103 \\
\hline Trial 7 & 108 & 110 & 104 & 102 & 102 & 108 \\
\hline Trial 8 & 101 & 100 & 108 & 109 & 103 & 105 \\
\hline Trial 9 & 107 & 106 & 109 & 109 & 102 & 102 \\
\hline Trial 10 & 102 & 100 & 98 & 98 & 99 & 102 \\
\hline Trial 11 & 93 & 96 & 90 & 90 & 101 & 96 \\
\hline Trial 12 & 105 & 107 & 91 & 94 & 108 & 102 \\
\hline Trial 13 & 102 & 101 & 105 & 107 & 101 & 102 \\
\hline Trial 14 & 97 & 99 & 95 & 92 & 109 & 101 \\
\hline Trial 15 & 95 & 96 & 93 & 91 & 106 & 96 \\
\hline
\end{tabular}

\begin{tabular}{|l|c|c|c|c|c|c|}
\hline $\begin{array}{l}\text { Subject 3 } \\
\text { MAP }\end{array}$ & $\mathrm{AI}$ & $\Delta \mathrm{T} / \mathrm{T}$ & $\mathrm{SI}$ & $\mathrm{RI}$ & MAA & Omron \\
\hline Trial 1 & 84 & 84 & 82 & 82 & 90 & 86 \\
\hline Trial 2 & 79 & 78 & 74 & 85 & 88 & 82 \\
\hline Trial 3 & 74 & 74 & 78 & 76 & 84 & 80 \\
\hline Trial 4 & 75 & 75 & 79 & 79 & 85 & 77 \\
\hline Trial 5 & 84 & 84 & 87 & 87 & 93 & 89 \\
\hline Trial 6 & 81 & 81 & 86 & 86 & 85 & 84 \\
\hline Trial 7 & 81 & 86 & 91 & 94 & 85 & 85 \\
\hline Trial 8 & 78 & 76 & 82 & 84 & 86 & 82 \\
\hline Trial 9 & 81 & 81 & 87 & 89 & 81 & 84 \\
\hline Trial 10 & 76 & 71 & 74 & 73 & 82 & 81 \\
\hline Trial 11 & 77 & 76 & 77 & 76 & 83 & 77 \\
\hline Trial 12 & 78 & 78 & 78 & 77 & 88 & 79 \\
\hline Trial 13 & 71 & 70 & 69 & 78 & 85 & 79 \\
\hline Trial 14 & 78 & 74 & 78 & 77 & 82 & 80 \\
\hline Trial 15 & 82 & 82 & 81 & 83 & 83 & 79 \\
\hline
\end{tabular}

\begin{tabular}{|l|c|c|c|c|c|c|}
\hline $\begin{array}{l}\text { Subject 3 } \\
\text { DBP }\end{array}$ & AI & $\Delta \mathrm{T} / \mathrm{T}$ & SI & RI & MAA & Omron \\
\hline Trial 1 & 80 & 82 & 84 & 84 & 80 & 77 \\
\hline Trial 2 & 74 & 75 & 77 & 75 & 76 & 73 \\
\hline Trial 3 & 68 & 66 & 66 & 68 & 71 & 71 \\
\hline Trial 4 & 68 & 67 & 65 & 68 & 72 & 68 \\
\hline Trial 5 & 84 & 85 & 85 & 85 & 80 & 79 \\
\hline Trial 6 & 69 & 69 & 72 & 71 & 76 & 75 \\
\hline Trial 7 & 73 & 72 & 75 & 72 & 74 & 73 \\
\hline Trial 8 & 76 & 77 & 79 & 78 & 76 & 70 \\
\hline Trial 9 & 75 & 76 & 76 & 77 & 69 & 75 \\
\hline Trial 10 & 65 & 66 & 67 & 65 & 71 & 71 \\
\hline Trial 11 & 67 & 68 & 69 & 68 & 71 & 67 \\
\hline Trial 12 & 75 & 77 & 72 & 71 & 75 & 68 \\
\hline Trial 13 & 66 & 68 & 66 & 65 & 73 & 68 \\
\hline Trial 14 & 70 & 71 & 73 & 71 & 71 & 70 \\
\hline Trial 15 & 78 & 78 & 80 & 76 & 71 & 71 \\
\hline
\end{tabular}

\begin{tabular}{|l|c|c|c|c|c|c|}
\hline $\begin{array}{l}\text { Subject 4 } \\
\text { SBP }\end{array}$ & AI & $\Delta \mathrm{T} / \mathrm{T}$ & $\mathrm{SI}$ & $\mathrm{RI}$ & $\mathrm{MAA}$ & Omron \\
\hline Trial 1 & 122 & 121 & 119 & 118 & 116 & 115 \\
\hline Trial 2 & 100 & 114 & 109 & 110 & 117 & 106 \\
\hline Trial 3 & 114 & 114 & 116 & 115 & 113 & 110 \\
\hline Trial 4 & 126 & 124 & 123 & 123 & 111 & 119 \\
\hline Trial 5 & 122 & 121 & 122 & 121 & 113 & 118 \\
\hline Trial 6 & 106 & 105 & 105 & 107 & 118 & 117 \\
\hline Trial 7 & 103 & 101 & 104 & 104 & 120 & 112 \\
\hline Trial 8 & 122 & 123 & 122 & 123 & 116 & 115 \\
\hline Trial 9 & 121 & 120 & 120 & 119 & 114 & 119 \\
\hline Trial 10 & 114 & 115 & 116 & 118 & 114 & 119 \\
\hline Trial 11 & 104 & 106 & 102 & 106 & 117 & 111 \\
\hline Trial 12 & 107 & 108 & 105 & 105 & 114 & 115 \\
\hline Trial 13 & 112 & 112 & 114 & 114 & 107 & 112 \\
\hline Trial 14 & 117 & 118 & 117 & 118 & 112 & 111 \\
\hline Trial 15 & 111 & 119 & 115 & 113 & 116 & 116 \\
\hline
\end{tabular}

\begin{tabular}{|l|c|c|c|c|c|c|}
\hline $\begin{array}{l}\text { Subject 4 } \\
\text { MAP }\end{array}$ & AI & $\Delta \mathrm{T} / \mathrm{T}$ & SI & RI & MAA & Omron \\
\hline Trial 1 & 90 & 90 & 93 & 94 & 92 & 89 \\
\hline Trial 2 & 81 & 83 & 87 & 87 & 88 & 86 \\
\hline Trial 3 & 88 & 85 & 81 & 88 & 91 & 90 \\
\hline Trial 4 & 94 & 94 & 95 & 90 & 89 & 92 \\
\hline Trial 5 & 90 & 91 & 91 & 90 & 92 & 91 \\
\hline Trial 6 & 90 & 90 & 92 & 93 & 88 & 93 \\
\hline Trial 7 & 84 & 81 & 86 & 86 & 86 & 90 \\
\hline Trial 8 & 84 & 80 & 85 & 84 & 88 & 90 \\
\hline Trial 9 & 90 & 90 & 91 & 92 & 87 & 92 \\
\hline Trial 10 & 87 & 84 & 89 & 92 & 84 & 96 \\
\hline Trial 11 & 96 & 91 & 99 & 101 & 89 & 90 \\
\hline Trial 12 & 87 & 85 & 83 & 81 & 84 & 89 \\
\hline Trial 13 & 92 & 94 & 94 & 81 & 82 & 83 \\
\hline Trial 14 & 86 & 81 & 94 & 81 & 85 & 84 \\
\hline Trial 15 & 87 & 89 & 87 & 87 & 85 & 87 \\
\hline
\end{tabular}

\begin{tabular}{|l|c|c|c|c|c|c|}
\hline $\begin{array}{l}\text { Subject 4 } \\
\text { DBP }\end{array}$ & AI & $\Delta \mathrm{T} / \mathrm{T}$ & $\mathrm{SI}$ & $\mathrm{RI}$ & MAA & Omron \\
\hline Trial 1 & 70 & 69 & 69 & 72 & 78 & 76 \\
\hline Trial 2 & 69 & 71 & 75 & 71 & 74 & 76 \\
\hline Trial 3 & 85 & 85 & 82 & 84 & 77 & 80 \\
\hline Trial 4 & 74 & 76 & 74 & 72 & 76 & 78 \\
\hline Trial 5 & 73 & 72 & 71 & 72 & 79 & 78 \\
\hline Trial 6 & 81 & 83 & 83 & 80 & 74 & 81 \\
\hline Trial 7 & 77 & 79 & 77 & 78 & 72 & 79 \\
\hline Trial 8 & 81 & 88 & 81 & 80 & 70 & 78 \\
\hline Trial 9 & 84 & 85 & 80 & 79 & 74 & 79 \\
\hline Trial 10 & 79 & 79 & 76 & 75 & 72 & 84 \\
\hline Trial 11 & 84 & 86 & 84 & 84 & 73 & 80 \\
\hline Trial 12 & 70 & 71 & 69 & 68 & 70 & 76 \\
\hline Trial 13 & 73 & 72 & 73 & 70 & 70 & 69 \\
\hline Trial 14 & 68 & 69 & 67 & 69 & 70 & 71 \\
\hline Trial 15 & 77 & 77 & 78 & 76 & 69 & 73 \\
\hline
\end{tabular}




\begin{tabular}{|l|c|c|c|c|c|c|}
\hline $\begin{array}{l}\text { Subject 5 } \\
\text { SBP }\end{array}$ & AI & $\Delta \mathrm{T} / \mathrm{T}$ & $\mathrm{SI}$ & $\mathrm{RI}$ & MAA & Omron \\
\hline Trial 1 & 110 & 112 & 114 & 112 & 116 & 109 \\
\hline Trial 2 & 98 & 99 & 98 & 99 & 110 & 106 \\
\hline Trial 3 & 101 & 101 & 105 & 104 & 120 & 108 \\
\hline Trial 4 & 106 & 107 & 105 & 107 & 115 & 99 \\
\hline Trial 5 & 105 & 105 & 107 & 108 & 116 & 105 \\
\hline Trial 6 & 110 & 111 & 109 & 110 & 114 & 113 \\
\hline Trial 7 & 120 & 121 & 123 & 121 & 117 & 118 \\
\hline Trial 8 & 117 & 119 & 118 & 119 & 114 & 115 \\
\hline Trial 9 & 122 & 122 & 124 & 127 & 113 & 118 \\
\hline Trial 10 & 118 & 123 & 118 & 122 & 113 & 114 \\
\hline Trial 11 & 115 & 117 & 119 & 121 & 110 & 111 \\
\hline Trial 12 & 106 & 108 & 106 & 107 & 116 & 117 \\
\hline Trial 13 & 119 & 121 & 122 & 119 & 118 & 109 \\
\hline Trial 14 & 108 & 109 & 110 & 109 & 117 & 104 \\
\hline Trial 15 & 105 & 108 & 107 & 107 & 115 & 105 \\
\hline
\end{tabular}

\begin{tabular}{|l|c|c|c|c|c|c|}
\hline $\begin{array}{l}\text { Subject 5 } \\
\text { MAP }\end{array}$ & $\mathrm{AI}$ & $\Delta \mathrm{T} / \mathrm{T}$ & $\mathrm{SI}$ & $\mathrm{RI}$ & MAA & Omron \\
\hline Trial 1 & 80 & 81 & 82 & 81 & 93 & 84 \\
\hline Trial 2 & 78 & 78 & 79 & 80 & 85 & 81 \\
\hline Trial 3 & 78 & 80 & 84 & 85 & 90 & 83 \\
\hline Trial 4 & 70 & 76 & 78 & 76 & 91 & 78 \\
\hline Trial 5 & 91 & 93 & 83 & 83 & 93 & 82 \\
\hline Trial 6 & 90 & 90 & 89 & 88 & 92 & 88 \\
\hline Trial 7 & 90 & 89 & 94 & 92 & 92 & 90 \\
\hline Trial 8 & 81 & 82 & 87 & 87 & 91 & 87 \\
\hline Trial 9 & 85 & 85 & 86 & 89 & 90 & 88 \\
\hline Trial 10 & 89 & 89 & 86 & 81 & 89 & 87 \\
\hline Trial 11 & 84 & 83 & 89 & 89 & 87 & 86 \\
\hline Trial 12 & 85 & 84 & 88 & 86 & 91 & 88 \\
\hline Trial 13 & 86 & 86 & 84 & 85 & 93 & 88 \\
\hline Trial 14 & 81 & 86 & 83 & 83 & 91 & 83 \\
\hline Trial 15 & 84 & 84 & 90 & 90 & 90 & 88 \\
\hline
\end{tabular}

\begin{tabular}{|l|c|c|c|c|c|c|}
\hline $\begin{array}{l}\text { Subject 5 } \\
\text { DBP }\end{array}$ & AI & $\Delta \mathrm{T} / \mathrm{T}$ & SI & RI & MAA & Omron \\
\hline Trial 1 & 70 & 68 & 68 & 69 & 79 & 72 \\
\hline Trial 2 & 66 & 64 & 67 & 66 & 75 & 69 \\
\hline Trial 3 & 74 & 71 & 76 & 76 & 78 & 71 \\
\hline Trial 4 & 72 & 70 & 77 & 73 & 79 & 68 \\
\hline Trial 5 & 69 & 69 & 78 & 79 & 79 & 70 \\
\hline Trial 6 & 81 & 80 & 83 & 80 & 81 & 75 \\
\hline Trial 7 & 76 & 77 & 77 & 77 & 80 & 76 \\
\hline Trial 8 & 71 & 73 & 71 & 72 & 78 & 73 \\
\hline Trial 9 & 79 & 80 & 76 & 77 & 79 & 73 \\
\hline Trial 10 & 73 & 75 & 77 & 77 & 78 & 73 \\
\hline Trial 11 & 71 & 71 & 72 & 71 & 76 & 73 \\
\hline Trial 12 & 76 & 72 & 75 & 76 & 80 & 74 \\
\hline Trial 13 & 77 & 76 & 79 & 78 & 81 & 77 \\
\hline Trial 14 & 71 & 69 & 70 & 69 & 79 & 73 \\
\hline Trial 15 & 83 & 84 & 81 & 83 & 79 & 79 \\
\hline
\end{tabular}

\begin{tabular}{|l|c|c|c|c|c|c|}
\hline $\begin{array}{l}\text { Subject 6 } \\
\text { SBP }\end{array}$ & AI & $\Delta \mathrm{T} / \mathrm{T}$ & $\mathrm{SI}$ & $\mathrm{RI}$ & MAA & Omron \\
\hline Trial 1 & 122 & 123 & 122 & 124 & 108 & 117 \\
\hline Trial 2 & 109 & 110 & 114 & 110 & 107 & 109 \\
\hline Trial 3 & 100 & 99 & 101 & 101 & 110 & 104 \\
\hline Trial 4 & 110 & 113 & 115 & 113 & 106 & 110 \\
\hline Trial 5 & 109 & 111 & 108 & 107 & 108 & 105 \\
\hline Trial 6 & 124 & 118 & 125 & 125 & 106 & 118 \\
\hline Trial 7 & 112 & 114 & 116 & 113 & 115 & 119 \\
\hline Trial 8 & 119 & 124 & 120 & 122 & 113 & 119 \\
\hline Trial 9 & 117 & 117 & 119 & 121 & 110 & 113 \\
\hline Trial 10 & 126 & 123 & 127 & 126 & 107 & 120 \\
\hline Trial 11 & 119 & 119 & 117 & 116 & 110 & 112 \\
\hline Trial 12 & 116 & 118 & 119 & 118 & 110 & 116 \\
\hline Trial 13 & 111 & 114 & 115 & 111 & 107 & 107 \\
\hline Trial 14 & 114 & 113 & 115 & 112 & 104 & 107 \\
\hline Trial 15 & 114 & 112 & 113 & 114 & 107 & 114 \\
\hline
\end{tabular}

\begin{tabular}{|l|c|c|c|c|c|c|}
\hline $\begin{array}{l}\text { Subject 6 } \\
\text { MAP }\end{array}$ & AI & $\Delta \mathrm{T} / \mathrm{T}$ & $\mathrm{SI}$ & $\mathrm{RI}$ & MAA & Omron \\
\hline Trial 1 & 81 & 82 & 86 & 83 & 84 & 84 \\
\hline Trial 2 & 79 & 83 & 81 & 80 & 82 & 82 \\
\hline Trial 3 & 79 & 81 & 85 & 77 & 85 & 79 \\
\hline Trial 4 & 83 & 83 & 80 & 74 & 84 & 78 \\
\hline Trial 5 & 86 & 85 & 83 & 84 & 84 & 84 \\
\hline Trial 6 & 86 & 88 & 84 & 85 & 84 & 86 \\
\hline Trial 7 & 84 & 84 & 89 & 90 & 82 & 87 \\
\hline Trial 8 & 82 & 81 & 85 & 86 & 82 & 86 \\
\hline Trial 9 & 84 & 85 & 83 & 84 & 83 & 85 \\
\hline Trial 10 & 93 & 93 & 90 & 87 & 82 & 89 \\
\hline Trial 11 & 79 & 78 & 84 & 88 & 84 & 85 \\
\hline Trial 12 & 82 & 83 & 86 & 82 & 86 & 82 \\
\hline Trial 13 & 77 & 77 & 87 & 78 & 82 & 80 \\
\hline Trial 14 & 80 & 79 & 79 & 83 & 80 & 80 \\
\hline Trial 15 & 82 & 82 & 82 & 81 & 82 & 81 \\
\hline
\end{tabular}

\begin{tabular}{|l|c|c|c|c|c|c|}
\hline $\begin{array}{l}\text { Subject 6 } \\
\text { DBP }\end{array}$ & AI & $\Delta \mathrm{T} / \mathrm{T}$ & $\mathrm{SI}$ & $\mathrm{RI}$ & $\mathrm{MAA}$ & Omron \\
\hline Trial 1 & 74 & 73 & 74 & 73 & 70 & 68 \\
\hline Trial 2 & 74 & 73 & 73 & 76 & 68 & 68 \\
\hline Trial 3 & 69 & 70 & 70 & 71 & 71 & 67 \\
\hline Trial 4 & 68 & 71 & 68 & 70 & 70 & 62 \\
\hline Trial 5 & 76 & 77 & 70 & 75 & 70 & 73 \\
\hline Trial 6 & 65 & 67 & 64 & 66 & 70 & 70 \\
\hline Trial 7 & 70 & 73 & 74 & 74 & 69 & 71 \\
\hline Trial 8 & 68 & 67 & 66 & 67 & 67 & 70 \\
\hline Trial 9 & 79 & 82 & 77 & 77 & 67 & 71 \\
\hline Trial 10 & 80 & 79 & 78 & 79 & 67 & 73 \\
\hline Trial 11 & 79 & 80 & 80 & 78 & 70 & 72 \\
\hline Trial 12 & 70 & 71 & 72 & 70 & 70 & 65 \\
\hline Trial 13 & 67 & 66 & 65 & 63 & 68 & 66 \\
\hline Trial 14 & 69 & 67 & 68 & 68 & 66 & 66 \\
\hline Trial 15 & 72 & 73 & 71 & 72 & 69 & 65 \\
\hline
\end{tabular}




\begin{tabular}{|l|c|c|c|c|c|c|}
\hline $\begin{array}{l}\text { Subject 7 } \\
\text { SBP }\end{array}$ & AI & $\Delta \mathrm{T} / \mathrm{T}$ & $\mathrm{SI}$ & $\mathrm{RI}$ & $\mathrm{MAA}$ & Omron \\
\hline Trial 1 & 120 & 122 & 130 & 129 & 122 & 127 \\
\hline Trial 2 & 129 & 125 & 126 & 124 & 118 & 121 \\
\hline Trial 3 & 120 & 124 & 122 & 123 & 108 & 116 \\
\hline Trial 4 & 118 & 123 & 119 & 119 & 108 & 111 \\
\hline Trial 5 & 121 & 121 & 124 & 122 & 125 & 119 \\
\hline Trial 6 & 119 & 116 & 110 & 111 & 113 & 113 \\
\hline Trial 7 & 122 & 120 & 125 & 122 & 116 & 118 \\
\hline Trial 8 & 108 & 110 & 109 & 109 & 110 & 114 \\
\hline Trial 9 & 106 & 109 & 110 & 110 & 108 & 115 \\
\hline Trial 10 & 117 & 119 & 121 & 123 & 109 & 115 \\
\hline
\end{tabular}

\begin{tabular}{|l|c|c|c|c|c|c|}
\hline $\begin{array}{l}\text { Subject 7 } \\
\text { MAP }\end{array}$ & AI & $\Delta \mathrm{T} / \mathrm{T}$ & SI & RI & MAA & Omron \\
\hline Trial 1 & 92 & 90 & 94 & 94 & 94 & 99 \\
\hline Trial 2 & 87 & 88 & 93 & 90 & 91 & 95 \\
\hline Trial 3 & 86 & 86 & 88 & 87 & 90 & 92 \\
\hline Trial 4 & 94 & 95 & 91 & 92 & 96 & 89 \\
\hline Trial 5 & 87 & 88 & 94 & 94 & 86 & 92 \\
\hline Trial 6 & 80 & 83 & 85 & 85 & 83 & 89 \\
\hline Trial 7 & 92 & 96 & 93 & 93 & 96 & 89 \\
\hline Trial 8 & 92 & 95 & 90 & 89 & 96 & 89 \\
\hline Trial 9 & 92 & 96 & 86 & 88 & 95 & 89 \\
\hline Trial 10 & 90 & 91 & 87 & 88 & 90 & 93 \\
\hline
\end{tabular}

\begin{tabular}{|l|c|c|c|c|c|c|}
\hline $\begin{array}{l}\text { Subject 7 } \\
\text { DBP }\end{array}$ & AI & $\Delta \mathrm{T} / \mathrm{T}$ & SI & RI & MAA & Omron \\
\hline Trial 1 & 80 & 78 & 77 & 76 & 84 & 85 \\
\hline Trial 2 & 88 & 89 & 86 & 86 & 86 & 82 \\
\hline Trial 3 & 85 & 86 & 88 & 86 & 88 & 81 \\
\hline Trial 4 & 83 & 84 & 84 & 85 & 70 & 78 \\
\hline Trial 5 & 84 & 85 & 83 & 85 & 75 & 79 \\
\hline Trial 6 & 79 & 80 & 79 & 81 & 78 & 78 \\
\hline Trial 7 & 76 & 79 & 80 & 79 & 78 & 75 \\
\hline Trial 8 & 79 & 80 & 81 & 80 & 77 & 77 \\
\hline Trial 9 & 78 & 80 & 82 & 78 & 77 & 77 \\
\hline Trial 10 & 83 & 85 & 83 & 86 & 80 & 82 \\
\hline
\end{tabular}

\begin{tabular}{|l|c|c|c|c|c|c|}
\hline $\begin{array}{l}\text { Subject 8 } \\
\text { SBP }\end{array}$ & AI & $\Delta \mathrm{T} / \mathrm{T}$ & $\mathrm{SI}$ & $\mathrm{RI}$ & MAA & Omron \\
\hline Trial 1 & 100 & 102 & 104 & 107 & 110 & 116 \\
\hline Trial 2 & 108 & 107 & 104 & 106 & 115 & 117 \\
\hline Trial 3 & 109 & 107 & 106 & 107 & 101 & 102 \\
\hline Trial 4 & 115 & 115 & 112 & 112 & 100 & 107 \\
\hline Trial 5 & 102 & 104 & 105 & 105 & 99 & 102 \\
\hline Trial 6 & 103 & 106 & 101 & 104 & 104 & 100 \\
\hline Trial 7 & 120 & 122 & 119 & 119 & 115 & 113 \\
\hline Trial 8 & 102 & 102 & 104 & 105 & 112 & 110 \\
\hline Trial 9 & 112 & 114 & 110 & 109 & 115 & 115 \\
\hline Trial 10 & 109 & 107 & 109 & 109 & 107 & 111 \\
\hline
\end{tabular}

\begin{tabular}{|l|c|c|c|c|c|c|}
\hline $\begin{array}{l}\text { Subject 8 } \\
\text { MAP }\end{array}$ & AI & $\Delta \mathrm{T} / \mathrm{T}$ & SI & RI & MAA & Omron \\
\hline Trial 1 & 89 & 88 & 92 & 94 & 90 & 93 \\
\hline Trial 2 & 92 & 93 & 96 & 95 & 88 & 95 \\
\hline Trial 3 & 85 & 87 & 89 & 88 & 87 & 87 \\
\hline Trial 4 & 93 & 96 & 89 & 90 & 79 & 89 \\
\hline Trial 5 & 86 & 89 & 87 & 88 & 80 & 84 \\
\hline Trial 6 & 90 & 91 & 90 & 90 & 93 & 88 \\
\hline Trial 7 & 96 & 97 & 96 & 96 & 96 & 92 \\
\hline Trial 8 & 96 & 97 & 96 & 96 & 85 & 92 \\
\hline Trial 9 & 98 & 99 & 99 & 99 & 88 & 91 \\
\hline Trial 10 & 90 & 93 & 94 & 97 & 96 & 93 \\
\hline
\end{tabular}

\begin{tabular}{|l|c|c|c|c|c|c|}
\hline $\begin{array}{l}\text { Subject } 8 \\
\text { DBP }\end{array}$ & AI & $\Delta \mathrm{T} / \mathrm{T}$ & $\mathrm{SI}$ & $\mathrm{RI}$ & MAA & Omron \\
\hline Trial 1 & 90 & 92 & 89 & 87 & 80 & 82 \\
\hline Trial 2 & 91 & 90 & 91 & 91 & 79 & 85 \\
\hline Trial 3 & 88 & 90 & 87 & 86 & 86 & 80 \\
\hline Trial 4 & 90 & 90 & 86 & 85 & 86 & 80 \\
\hline Trial 5 & 82 & 81 & 78 & 80 & 74 & 76 \\
\hline Trial 6 & 84 & 86 & 83 & 83 & 81 & 82 \\
\hline Trial 7 & 89 & 87 & 86 & 86 & 80 & 82 \\
\hline Trial 8 & 86 & 90 & 93 & 90 & 80 & 83 \\
\hline Trial 9 & 76 & 74 & 76 & 72 & 86 & 80 \\
\hline Trial 10 & 82 & 81 & 86 & 80 & 79 & 84 \\
\hline
\end{tabular}

\begin{tabular}{|l|c|c|c|c|c|c|}
\hline $\begin{array}{l}\text { Subject 9 } \\
\text { SBP }\end{array}$ & AI & $\Delta \mathrm{T} / \mathrm{T}$ & SI & RI & MAA & Omron \\
\hline Trial 1 & 100 & 98 & 99 & 99 & 112 & 104 \\
\hline Trial 2 & 98 & 99 & 102 & 99 & 98 & 94 \\
\hline Trial 3 & 106 & 107 & 108 & 107 & 102 & 99 \\
\hline Trial 4 & 107 & 106 & 105 & 106 & 105 & 100 \\
\hline Trial 5 & 101 & 103 & 104 & 101 & 94 & 94 \\
\hline Trial 6 & 99 & 102 & 103 & 102 & 96 & 99 \\
\hline Trial 7 & 105 & 106 & 103 & 104 & 96 & 99 \\
\hline Trial 8 & 107 & 109 & 108 & 107 & 109 & 103 \\
\hline Trial 9 & 110 & 109 & 112 & 110 & 110 & 102 \\
\hline Trial 10 & 105 & 106 & 109 & 107 & 112 & 94 \\
\hline
\end{tabular}

\begin{tabular}{|l|c|c|c|c|c|c|}
\hline $\begin{array}{l}\text { Subject 9 } \\
\text { MAP }\end{array}$ & AI & $\Delta \mathrm{T} / \mathrm{T}$ & $\mathrm{SI}$ & $\mathrm{RI}$ & MAA & Omron \\
\hline Trial 1 & 76 & 77 & 78 & 79 & 89 & 78 \\
\hline Trial 2 & 70 & 70 & 71 & 72 & 75 & 71 \\
\hline Trial 3 & 74 & 73 & 75 & 76 & 71 & 74 \\
\hline Trial 4 & 77 & 76 & 75 & 75 & 72 & 76 \\
\hline Trial 5 & 73 & 74 & 77 & 79 & 69 & 75 \\
\hline Trial 6 & 81 & 80 & 83 & 82 & 74 & 79 \\
\hline Trial 7 & 78 & 76 & 79 & 76 & 74 & 76 \\
\hline Trial 8 & 79 & 80 & 79 & 80 & 73 & 77 \\
\hline Trial 9 & 78 & 81 & 79 & 80 & 72 & 77 \\
\hline Trial 10 & 76 & 79 & 77 & 78 & 70 & 74 \\
\hline
\end{tabular}




\begin{tabular}{|l|c|c|c|c|c|c|}
\hline $\begin{array}{l}\text { Subject 9 } \\
\text { DBP }\end{array}$ & AI & $\Delta \mathrm{T} / \mathrm{T}$ & SI & RI & MAA & Omron \\
\hline Trial 1 & 62 & 60 & 61 & 62 & 90 & 65 \\
\hline Trial 2 & 61 & 63 & 59 & 63 & 62 & 60 \\
\hline Trial 3 & 60 & 59 & 60 & 59 & 66 & 62 \\
\hline Trial 4 & 66 & 68 & 68 & 68 & 66 & 64 \\
\hline Trial 5 & 68 & 69 & 69 & 68 & 65 & 66 \\
\hline Trial 6 & 74 & 76 & 76 & 74 & 73 & 69 \\
\hline Trial 7 & 71 & 70 & 71 & 70 & 71 & 65 \\
\hline Trial 8 & 74 & 71 & 73 & 71 & 70 & 64 \\
\hline Trial 9 & 70 & 72 & 70 & 72 & 69 & 65 \\
\hline Trial 10 & 71 & 70 & 70 & 70 & 67 & 64 \\
\hline
\end{tabular}

\begin{tabular}{|l|c|c|c|c|c|c|}
\hline $\begin{array}{l}\text { Subject 10 } \\
\text { SBP }\end{array}$ & AI & $\Delta \mathrm{T} / \mathrm{T}$ & SI & RI & MAA & Omron \\
\hline Trial 1 & 100 & 99 & 105 & 107 & 110 & 104 \\
\hline Trial 2 & 109 & 110 & 108 & 107 & 112 & 104 \\
\hline Trial 3 & 120 & 121 & 120 & 123 & 118 & 113 \\
\hline Trial 4 & 107 & 109 & 107 & 106 & 108 & 98 \\
\hline Trial 5 & 101 & 103 & 103 & 105 & 101 & 107 \\
\hline Trial 6 & 114 & 116 & 116 & 114 & 96 & 101 \\
\hline Trial 7 & 112 & 114 & 114 & 112 & 98 & 105 \\
\hline Trial 8 & 109 & 109 & 107 & 105 & 101 & 101 \\
\hline Trial 9 & 107 & 107 & 102 & 104 & 104 & 103 \\
\hline Trial 10 & 101 & 100 & 101 & 99 & 109 & 107 \\
\hline
\end{tabular}

\begin{tabular}{|l|c|c|c|c|c|c|}
\hline $\begin{array}{l}\text { Subject 10 } \\
\text { MAP }\end{array}$ & $\mathrm{AI}$ & $\Delta \mathrm{T} / \mathrm{T}$ & $\mathrm{SI}$ & $\mathrm{RI}$ & MAA & Omron \\
\hline Trial 1 & 80 & 83 & 84 & 83 & 89 & 82 \\
\hline Trial 2 & 79 & 80 & 79 & 79 & 74 & 78 \\
\hline Trial 3 & 82 & 85 & 81 & 86 & 84 & 84 \\
\hline Trial 4 & 79 & 82 & 78 & 81 & 77 & 80 \\
\hline Trial 5 & 85 & 86 & 87 & 84 & 85 & 83 \\
\hline Trial 6 & 76 & 77 & 78 & 77 & 79 & 75 \\
\hline Trial 7 & 80 & 83 & 83 & 82 & 83 & 81 \\
\hline Trial 8 & 79 & 80 & 81 & 80 & 79 & 77 \\
\hline Trial 9 & 78 & 77 & 79 & 79 & 77 & 76 \\
\hline Trial 10 & 83 & 81 & 77 & 76 & 79 & 80 \\
\hline
\end{tabular}

\begin{tabular}{|l|c|c|c|c|c|c|}
\hline $\begin{array}{l}\text { Subject 10 } \\
\text { DBP }\end{array}$ & AI & $\Delta \mathrm{T} / \mathrm{T}$ & SI & RI & MAA & Omron \\
\hline Trial 1 & 73 & 73 & 74 & 72 & 77 & 71 \\
\hline Trial 2 & 67 & 69 & 68 & 67 & 73 & 65 \\
\hline Trial 3 & 72 & 71 & 70 & 73 & 71 & 70 \\
\hline Trial 4 & 73 & 74 & 72 & 72 & 70 & 71 \\
\hline Trial 5 & 70 & 69 & 68 & 69 & 70 & 71 \\
\hline Trial 6 & 65 & 66 & 67 & 66 & 69 & 63 \\
\hline Trial 7 & 69 & 68 & 67 & 67 & 68 & 70 \\
\hline Trial 8 & 63 & 64 & 62 & 61 & 69 & 66 \\
\hline Trial 9 & 60 & 61 & 62 & 61 & 61 & 63 \\
\hline Trial 10 & 65 & 67 & 69 & 67 & 63 & 67 \\
\hline
\end{tabular}

\begin{tabular}{|l|c|c|c|c|c|c|}
\hline $\begin{array}{l}\text { Subject 11 } \\
\text { SBP }\end{array}$ & $\mathrm{AI}$ & $\Delta \mathrm{T} / \mathrm{T}$ & $\mathrm{SI}$ & $\mathrm{RI}$ & MAA & Omron \\
\hline Trial 1 & 106 & 105 & 104 & 105 & 95 & 96 \\
\hline Trial 2 & 104 & 101 & 100 & 103 & 93 & 89 \\
\hline Trial 3 & 98 & 99 & 99 & 97 & 92 & 95 \\
\hline Trial 4 & 101 & 98 & 100 & 102 & 89 & 91 \\
\hline Trial 5 & 103 & 100 & 101 & 100 & 96 & 94 \\
\hline Trial 6 & 96 & 96 & 98 & 97 & 91 & 88 \\
\hline Trial 7 & 92 & 90 & 91 & 93 & 88 & 84 \\
\hline Trial 8 & 89 & 88 & 87 & 87 & 85 & 82 \\
\hline Trial 9 & 88 & 86 & 87 & 85 & 88 & 86 \\
\hline Trial 10 & 88 & 85 & 86 & 87 & 86 & 83 \\
\hline
\end{tabular}

\begin{tabular}{|l|c|c|c|c|c|c|}
\hline $\begin{array}{l}\text { Subject 11 } \\
\text { MAP }\end{array}$ & AI & $\Delta \mathrm{T} / \mathrm{T}$ & SI & RI & MAA & Omron \\
\hline Trial 1 & 72 & 72 & 73 & 73 & 71 & 74 \\
\hline Trial 2 & 70 & 72 & 71 & 70 & 74 & 69 \\
\hline Trial 3 & 71 & 70 & 69 & 69 & 69 & 73 \\
\hline Trial 4 & 70 & 73 & 73 & 73 & 79 & 68 \\
\hline Trial 5 & 66 & 67 & 72 & 71 & 81 & 73 \\
\hline Trial 6 & 68 & 68 & 69 & 71 & 75 & 66 \\
\hline Trial 7 & 69 & 67 & 68 & 65 & 74 & 66 \\
\hline Trial 8 & 61 & 61 & 63 & 62 & 69 & 64 \\
\hline Trial 9 & 68 & 66 & 64 & 61 & 66 & 66 \\
\hline Trial 10 & 64 & 62 & 60 & 60 & 64 & 65 \\
\hline
\end{tabular}

\begin{tabular}{|l|c|c|c|c|c|c|}
\hline $\begin{array}{l}\text { Subject 11 } \\
\text { DBP }\end{array}$ & AI & $\Delta \mathrm{T} / \mathrm{T}$ & SI & RI & MAA & Omron \\
\hline Trial 1 & 71 & 70 & 72 & 70 & 69 & 64 \\
\hline Trial 2 & 54 & 55 & 57 & 56 & 55 & 59 \\
\hline Trial 3 & 77 & 76 & 75 & 77 & 70 & 62 \\
\hline Trial 4 & 76 & 74 & 71 & 75 & 70 & 57 \\
\hline Trial 5 & 75 & 72 & 73 & 72 & 71 & 63 \\
\hline Trial 6 & 62 & 60 & 59 & 61 & 58 & 56 \\
\hline Trial 7 & 65 & 66 & 63 & 68 & 59 & 58 \\
\hline Trial 8 & 60 & 62 & 59 & 62 & 55 & 55 \\
\hline Trial 9 & 63 & 63 & 61 & 61 & 54 & 57 \\
\hline Trial 10 & 63 & 64 & 62 & 62 & 66 & 57 \\
\hline
\end{tabular}




\begin{tabular}{|l|c|c|c|c|c|c|}
\hline $\begin{array}{l}\text { Subject 12 } \\
\text { SBP }\end{array}$ & AI & $\Delta \mathrm{T} / \mathrm{T}$ & SI & RI & MAA & Omron \\
\hline Trial 1 & 104 & 102 & 107 & 109 & 110 & 102 \\
\hline Trial 2 & 95 & 96 & 99 & 100 & 98 & 96 \\
\hline Trial 3 & 92 & 94 & 91 & 89 & 98 & 101 \\
\hline Trial 4 & 97 & 99 & 98 & 99 & 105 & 99 \\
\hline Trial 5 & 102 & 103 & 106 & 106 & 110 & 110 \\
\hline
\end{tabular}

\begin{tabular}{|l|c|c|c|c|c|c|}
\hline $\begin{array}{l}\text { Subject 12 } \\
\text { MAP }\end{array}$ & AI & $\Delta \mathrm{T} / \mathrm{T}$ & SI & RI & MAA & Omron \\
\hline Trial 1 & 72 & 71 & 75 & 74 & 79 & 76 \\
\hline Trial 2 & 76 & 77 & 72 & 73 & 70 & 70 \\
\hline Trial 3 & 70 & 72 & 74 & 73 & 77 & 75 \\
\hline Trial 4 & 79 & 80 & 74 & 76 & 76 & 71 \\
\hline Trial 5 & 74 & 73 & 76 & 76 & 70 & 78 \\
\hline
\end{tabular}

\begin{tabular}{|l|c|c|c|c|c|c|}
\hline $\begin{array}{l}\text { Subject 12 } \\
\text { DBP }\end{array}$ & AI & $\Delta \mathrm{T} / \mathrm{T}$ & SI & RI & MAA & Omron \\
\hline Trial 1 & 61 & 62 & 68 & 66 & 59 & 64 \\
\hline Trial 2 & 60 & 63 & 64 & 65 & 61 & 58 \\
\hline Trial 3 & 66 & 65 & 69 & 66 & 69 & 62 \\
\hline Trial 4 & 64 & 68 & 60 & 62 & 64 & 57 \\
\hline Trial 5 & 59 & 59 & 61 & 60 & 69 & 62 \\
\hline
\end{tabular}

\begin{tabular}{|l|c|c|c|c|c|c|}
\hline $\begin{array}{l}\text { Subject 13 } \\
\text { SBP }\end{array}$ & AI & $\Delta \mathrm{T} / \mathrm{T}$ & SI & RI & MAA & Omron \\
\hline Trial 1 & 112 & 111 & 115 & 116 & 115 & 111 \\
\hline Trial 2 & 108 & 109 & 104 & 104 & 119 & 114 \\
\hline Trial 3 & 119 & 119 & 121 & 123 & 108 & 111 \\
\hline Trial 4 & 115 & 116 & 119 & 114 & 101 & 109 \\
\hline Trial 5 & 120 & 121 & 121 & 121 & 119 & 113 \\
\hline
\end{tabular}

\begin{tabular}{|l|c|c|c|c|c|c|}
\hline $\begin{array}{l}\text { Subject 13 } \\
\text { MAP }\end{array}$ & AI & $\Delta \mathrm{T} / \mathrm{T}$ & SI & RI & MAA & Omron \\
\hline Trial 1 & 80 & 79 & 79 & 80 & 78 & 82 \\
\hline Trial 2 & 81 & 81 & 86 & 85 & 86 & 84 \\
\hline Trial 3 & 82 & 82 & 83 & 83 & 83 & 83 \\
\hline Trial 4 & 82 & 82 & 83 & 83 & 83 & 83 \\
\hline Trial 5 & 90 & 91 & 85 & 86 & 89 & 86 \\
\hline
\end{tabular}

\begin{tabular}{|l|c|c|c|c|c|c|}
\hline $\begin{array}{l}\text { Subject 13 } \\
\text { DBP }\end{array}$ & AI & $\Delta \mathrm{T} / \mathrm{T}$ & SI & RI & MAA & Omron \\
\hline Trial 1 & 71 & 72 & 70 & 70 & 69 & 68 \\
\hline Trial 2 & 74 & 74 & 72 & 73 & 72 & 70 \\
\hline Trial 3 & 75 & 77 & 76 & 76 & 72 & 70 \\
\hline Trial 4 & 74 & 75 & 75 & 75 & 69 & 71 \\
\hline Trial 5 & 70 & 71 & 70 & 70 & 71 & 73 \\
\hline
\end{tabular}

\begin{tabular}{|l|c|c|c|c|c|c|}
\hline $\begin{array}{l}\text { Subject 14 } \\
\text { SBP }\end{array}$ & AI & $\Delta \mathrm{T} / \mathrm{T}$ & SI & RI & MAA & Omron \\
\hline Trial 1 & 122 & 120 & 109 & 111 & 125 & 119 \\
\hline Trial 2 & 118 & 116 & 112 & 112 & 122 & 120 \\
\hline Trial 3 & 126 & 128 & 130 & 128 & 126 & 124 \\
\hline Trial 4 & 117 & 115 & 126 & 126 & 122 & 120 \\
\hline Trial 5 & 115 & 113 & 120 & 121 & 119 & 118 \\
\hline
\end{tabular}

\begin{tabular}{|l|c|c|c|c|c|c|}
\hline $\begin{array}{l}\text { Subject 14 } \\
\text { MAP }\end{array}$ & AI & $\Delta \mathrm{T} / \mathrm{T}$ & SI & RI & MAA & Omron \\
\hline Trial 1 & 96 & 98 & 94 & 94 & 95 & 93 \\
\hline Trial 2 & 92 & 91 & 94 & 94 & 96 & 93 \\
\hline Trial 3 & 94 & 93 & 93 & 93 & 96 & 93 \\
\hline Trial 4 & 91 & 90 & 90 & 90 & 95 & 92 \\
\hline Trial 5 & 91 & 90 & 90 & 91 & 89 & 91 \\
\hline
\end{tabular}

\begin{tabular}{|l|c|c|c|c|c|c|}
\hline $\begin{array}{l}\text { Subject 14 } \\
\text { DBP }\end{array}$ & AI & $\Delta \mathrm{T} / \mathrm{T}$ & SI & RI & MAA & Omron \\
\hline Trial 1 & 78 & 76 & 72 & 76 & 79 & 80 \\
\hline Trial 2 & 78 & 77 & 70 & 77 & 79 & 80 \\
\hline Trial 3 & 65 & 64 & 68 & 66 & 69 & 78 \\
\hline Trial 4 & 61 & 62 & 64 & 63 & 65 & 79 \\
\hline Trial 5 & 75 & 76 & 79 & 78 & 71 & 78 \\
\hline
\end{tabular}

\begin{tabular}{|l|c|c|c|c|c|c|}
\hline $\begin{array}{l}\text { Subject 15 } \\
\text { SBP }\end{array}$ & AI & $\Delta \mathrm{T} / \mathrm{T}$ & SI & RI & MAA & Omron \\
\hline Trial 1 & 90 & 91 & 93 & 89 & 91 & 95 \\
\hline Trial 2 & 101 & 102 & 106 & 109 & 96 & 94 \\
\hline Trial 3 & 95 & 95 & 98 & 98 & 90 & 92 \\
\hline Trial 4 & 99 & 100 & 106 & 108 & 90 & 90 \\
\hline Trial 5 & 87 & 88 & 89 & 93 & 89 & 85 \\
\hline
\end{tabular}




\begin{tabular}{|l|c|c|c|c|c|c|}
\hline $\begin{array}{l}\text { Subject 15 } \\
\text { MAP }\end{array}$ & AI & $\Delta \mathrm{T} / \mathrm{T}$ & SI & RI & MAA & Omron \\
\hline Trial 1 & 68 & 69 & 70 & 70 & 70 & 72 \\
\hline Trial 2 & 71 & 70 & 71 & 71 & 70 & 72 \\
\hline Trial 3 & 68 & 68 & 67 & 66 & 68 & 69 \\
\hline Trial 4 & 72 & 71 & 73 & 74 & 69 & 70 \\
\hline Trial 5 & 60 & 63 & 62 & 64 & 64 & 69 \\
\hline
\end{tabular}

\begin{tabular}{|l|c|c|c|c|c|c|}
\hline $\begin{array}{l}\text { Subject 15 } \\
\text { DBP }\end{array}$ & AI & $\Delta \mathrm{T} / \mathrm{T}$ & SI & RI & MAA & Omron \\
\hline Trial 1 & 63 & 64 & 66 & 67 & 69 & 61 \\
\hline Trial 2 & 63 & 66 & 66 & 67 & 69 & 61 \\
\hline Trial 3 & 69 & 69 & 68 & 68 & 64 & 58 \\
\hline Trial 4 & 61 & 63 & 59 & 60 & 58 & 60 \\
\hline Trial 5 & 60 & 59 & 63 & 63 & 60 & 62 \\
\hline
\end{tabular}

\begin{tabular}{|l|c|c|c|c|c|c|}
\hline $\begin{array}{l}\text { Subject 16 } \\
\text { SBP }\end{array}$ & AI & $\Delta \mathrm{T} / \mathrm{T}$ & SI & RI & MAA & Omron \\
\hline Trial 1 & 121 & 120 & 123 & 124 & 125 & 120 \\
\hline Trial 2 & 124 & 124 & 123 & 122 & 122 & 114 \\
\hline Trial 3 & 105 & 101 & 108 & 106 & 119 & 115 \\
\hline Trial 4 & 108 & 106 & 107 & 106 & 109 & 111 \\
\hline Trial 5 & 105 & 101 & 104 & 102 & 106 & 105 \\
\hline
\end{tabular}

\begin{tabular}{|l|c|c|c|c|c|c|}
\hline $\begin{array}{l}\text { Subject 16 } \\
\text { MAP }\end{array}$ & AI & $\Delta \mathrm{T} / \mathrm{T}$ & SI & RI & MAA & Omron \\
\hline Trial 1 & 94 & 93 & 91 & 92 & 90 & 92 \\
\hline Trial 2 & 91 & 90 & 90 & 90 & 94 & 88 \\
\hline Trial 3 & 84 & 85 & 86 & 85 & 88 & 87 \\
\hline Trial 4 & 86 & 86 & 87 & 88 & 82 & 88 \\
\hline Trial 5 & 80 & 81 & 79 & 79 & 81 & 83 \\
\hline
\end{tabular}

\begin{tabular}{|l|c|c|c|c|c|c|}
\hline $\begin{array}{l}\text { Subject 16 } \\
\text { DBP }\end{array}$ & AI & $\Delta \mathrm{T} / \mathrm{T}$ & SI & RI & MAA & Omron \\
\hline Trial 1 & 68 & 71 & 73 & 75 & 70 & 78 \\
\hline Trial 2 & 68 & 68 & 71 & 71 & 71 & 76 \\
\hline Trial 3 & 75 & 77 & 79 & 76 & 76 & 73 \\
\hline Trial 4 & 70 & 69 & 68 & 67 & 74 & 77 \\
\hline Trial 5 & 60 & 61 & 66 & 65 & 62 & 72 \\
\hline
\end{tabular}

\begin{tabular}{|l|c|c|c|c|c|c|}
\hline $\begin{array}{l}\text { Subject } 17 \\
\text { SBP }\end{array}$ & AI & $\Delta \mathrm{T} / \mathrm{T}$ & SI & RI & MAA & Omron \\
\hline Trial 1 & 120 & 118 & 117 & 118 & 120 & 122 \\
\hline Trial 2 & 126 & 127 & 129 & 129 & 125 & 123 \\
\hline Trial 3 & 100 & 102 & 104 & 105 & 95 & 97 \\
\hline Trial 4 & 107 & 109 & 108 & 107 & 96 & 105 \\
\hline Trial 5 & 109 & 109 & 107 & 106 & 115 & 112 \\
\hline
\end{tabular}

\begin{tabular}{|l|c|c|c|c|c|c|}
\hline $\begin{array}{l}\text { Subject 17 } \\
\text { MAP }\end{array}$ & AI & $\Delta \mathrm{T} / \mathrm{T}$ & SI & RI & MAA & Omron \\
\hline Trial 1 & 96 & 95 & 90 & 92 & 93 & 91 \\
\hline Trial 2 & 97 & 98 & 94 & 94 & 89 & 92 \\
\hline Trial 3 & 89 & 88 & 82 & 82 & 86 & 81 \\
\hline Trial 4 & 88 & 88 & 94 & 96 & 88 & 90 \\
\hline Trial 5 & 88 & 87 & 87 & 89 & 81 & 88 \\
\hline
\end{tabular}

\begin{tabular}{|l|c|c|c|c|c|c|}
\hline $\begin{array}{l}\text { Subject 17 } \\
\text { DBP }\end{array}$ & AI & $\Delta \mathrm{T} / \mathrm{T}$ & SI & RI & MAA & Omron \\
\hline Trial 1 & 79 & 80 & 83 & 86 & 77 & 76 \\
\hline Trial 2 & 80 & 82 & 83 & 83 & 79 & 77 \\
\hline Trial 3 & 81 & 84 & 80 & 79 & 69 & 74 \\
\hline Trial 4 & 80 & 82 & 84 & 82 & 80 & 83 \\
\hline Trial 5 & 79 & 80 & 81 & 82 & 79 & 77 \\
\hline
\end{tabular}

\begin{tabular}{|l|c|c|c|c|c|c|}
\hline $\begin{array}{l}\text { Subject 18 } \\
\text { SBP }\end{array}$ & AI & $\Delta \mathrm{T} / \mathrm{T}$ & SI & RI & MAA & Omron \\
\hline Trial 1 & 114 & 116 & 117 & 118 & 115 & 113 \\
\hline Trial 2 & 119 & 122 & 123 & 122 & 122 & 120 \\
\hline Trial 3 & 119 & 120 & 121 & 122 & 119 & 114 \\
\hline Trial 4 & 118 & 118 & 119 & 121 & 116 & 115 \\
\hline Trial 5 & 110 & 109 & 108 & 108 & 112 & 112 \\
\hline
\end{tabular}

\begin{tabular}{|l|c|c|c|c|c|c|}
\hline $\begin{array}{l}\text { Subject 18 } \\
\text { MAP }\end{array}$ & AI & $\Delta \mathrm{T} / \mathrm{T}$ & SI & RI & MAA & Omron \\
\hline Trial 1 & 80 & 79 & 83 & 80 & 81 & 84 \\
\hline Trial 2 & 83 & 82 & 85 & 79 & 87 & 91 \\
\hline Trial 3 & 86 & 81 & 80 & 81 & 82 & 84 \\
\hline Trial 4 & 86 & 86 & 82 & 85 & 84 & 86 \\
\hline Trial 5 & 79 & 80 & 89 & 90 & 85 & 85 \\
\hline
\end{tabular}




\begin{tabular}{|l|c|c|c|c|c|c|}
\hline $\begin{array}{l}\text { Subject 18 } \\
\text { DBP }\end{array}$ & AI & $\Delta \mathrm{T} / \mathrm{T}$ & SI & RI & MAA & Omron \\
\hline Trial 1 & 68 & 67 & 60 & 59 & 69 & 70 \\
\hline Trial 2 & 74 & 73 & 71 & 72 & 77 & 77 \\
\hline Trial 3 & 66 & 67 & 62 & 62 & 68 & 70 \\
\hline Trial 4 & 61 & 64 & 63 & 61 & 69 & 72 \\
\hline Trial 5 & 61 & 62 & 63 & 64 & 69 & 72 \\
\hline
\end{tabular}




\section{APPENDIX B- BP Estimation Using Maximum Slope}

\begin{tabular}{|c|c|c|c|c|c|c|c|c|c|c|}
\hline & & \multicolumn{3}{|c|}{ MAX. Slope } & \multicolumn{3}{|c|}{ MAA } & \multicolumn{3}{|c|}{ OMRON } \\
\hline & & DBP & MAP & SBP & DBP & MAP & SBP & DBP & MAP & SBP \\
\hline \multirow{15}{*}{ 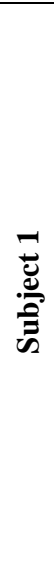 } & Trial 1 & 69 & 84 & 117 & 63 & 87 & 120 & 73 & 89 & 121 \\
\hline & Trial 2 & 70 & 85 & 128 & 66 & 82 & 121 & 74 & 90 & 121 \\
\hline & Trial 3 & 64 & 81 & 120 & 66 & 80 & 117 & 74 & 91 & 126 \\
\hline & Trial 4 & 70 & 96 & 117 & 66 & 82 & 111 & 74 & 91 & 126 \\
\hline & Trial 5 & 61 & 106 & 134 & 68 & 103 & 130 & 71 & 88 & 122 \\
\hline & Trial 6 & 70 & 88 & 119 & 69 & 94 & 125 & 75 & 92 & 127 \\
\hline & Trial 7 & 79 & 98 & 132 & 67 & 82 & 123 & 71 & 90 & 127 \\
\hline & Trial 8 & 83 & 99 & 139 & 70 & 94 & 124 & 78 & 95 & 130 \\
\hline & Trial 9 & 77 & 89 & 114 & 73 & 89 & 123 & 85 & 100 & 130 \\
\hline & Trial 10 & 72 & 91 & 128 & 69 & 86 & 128 & 75 & 94 & 132 \\
\hline & Trial 11 & 76 & 90 & 121 & 71 & 91 & 125 & 69 & 84 & 114 \\
\hline & Trial 12 & 74 & 80 & 118 & 74 & 94 & 123 & 69 & 84 & 113 \\
\hline & Trial 13 & 67 & 80 & 119 & 69 & 88 & 118 & 67 & 83 & 115 \\
\hline & Trial 14 & 60 & 90 & 123 & 74 & 88 & 118 & 70 & 87 & 122 \\
\hline & Trial 15 & 59 & 71 & 108 & 73 & 88 & 114 & 70 & 87 & 120 \\
\hline \multirow{15}{*}{ 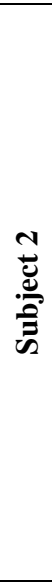 } & Trial 1 & 89 & 107 & 140 & 78 & 90 & 123 & 84 & 101 & 136 \\
\hline & Trial 2 & 76 & 91 & 124 & 80 & 92 & 122 & 80 & 96 & 129 \\
\hline & Trial 3 & 79 & 94 & 130 & 78 & 91 & 119 & 77 & 95 & 130 \\
\hline & Trial 4 & 84 & 104 & 122 & 76 & 88 & 120 & 79 & 92 & 119 \\
\hline & Trial 5 & 76 & 96 & 130 & 81 & 95 & 121 & 76 & 95 & 132 \\
\hline & Trial 6 & 83 & 97 & 132 & 85 & 100 & 127 & 80 & 97 & 131 \\
\hline & Trial 7 & 74 & 90 & 120 & 86 & 97 & 124 & 79 & 95 & 127 \\
\hline & Trial 8 & 76 & 93 & 125 & 84 & 97 & 123 & 76 & 91 & 120 \\
\hline & Trial 9 & 88 & 96 & 122 & 80 & 92 & 121 & 79 & 92 & 119 \\
\hline & Trial 10 & 85 & 101 & 129 & 83 & 96 & 122 & 86 & 99 & 126 \\
\hline & Trial 11 & 67 & 82 & 108 & 69 & 80 & 114 & 71 & 86 & 116 \\
\hline & Trial 12 & 71 & 80 & 109 & 74 & 88 & 116 & 78 & 91 & 117 \\
\hline & Trial 13 & 70 & 82 & 106 & 74 & 87 & 115 & 75 & 88 & 115 \\
\hline & Trial 14 & 77 & 92 & 118 & 75 & 86 & 112 & 74 & 87 & 114 \\
\hline & Trial 15 & 66 & 79 & 106 & 76 & 87 & 115 & 71 & 85 & 113 \\
\hline \multirow{15}{*}{ 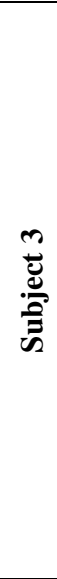 } & Trial 1 & 81 & 92 & 107 & 80 & 90 & 107 & 77 & 86 & 105 \\
\hline & Trial 2 & 83 & 93 & 112 & 76 & 88 & 108 & 73 & 82 & 100 \\
\hline & Trial 3 & 66 & 72 & 90 & 71 & 84 & 114 & 71 & 80 & 98 \\
\hline & Trial 4 & 79 & 85 & 103 & 72 & 85 & 106 & 68 & 77 & 96 \\
\hline & Trial 5 & 81 & 92 & 110 & 80 & 93 & 112 & 79 & 89 & 110 \\
\hline & Trial 6 & 70 & 77 & 97 & 76 & 85 & 104 & 75 & 84 & 103 \\
\hline & Trial 7 & 79 & 87 & 112 & 74 & 85 & 102 & 73 & 85 & 108 \\
\hline & Trial 8 & 77 & 93 & 114 & 76 & 86 & 103 & 70 & 82 & 105 \\
\hline & Trial 9 & 68 & 80 & 99 & 69 & 81 & 102 & 75 & 84 & 102 \\
\hline & Trial 10 & 65 & 74 & 97 & 71 & 82 & 99 & 71 & 81 & 102 \\
\hline & Trial 11 & 61 & 72 & 91 & 71 & 83 & 101 & 67 & 77 & 96 \\
\hline & Trial 12 & 63 & 74 & 90 & 75 & 88 & 108 & 68 & 79 & 102 \\
\hline & Trial 13 & 74 & 82 & 104 & 73 & 85 & 101 & 68 & 79 & 102 \\
\hline & Trial 14 & 79 & 88 & 112 & 71 & 82 & 109 & 70 & 80 & 101 \\
\hline & Trial 15 & 74 & 82 & 99 & 71 & 83 & 106 & 71 & 79 & 96 \\
\hline \multirow{7}{*}{ 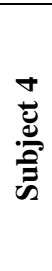 } & Trial 1 & 70 & 81 & 108 & 78 & 92 & 116 & 76 & 89 & 115 \\
\hline & Trial 2 & 81 & 90 & 112 & 74 & 88 & 117 & 76 & 86 & 106 \\
\hline & Trial 3 & 85 & 96 & 117 & 77 & 91 & 113 & 80 & 90 & 110 \\
\hline & Trial 4 & 84 & 96 & 123 & 76 & 89 & 111 & 78 & 92 & 119 \\
\hline & Trial 5 & 89 & 102 & 128 & 79 & 92 & 113 & 78 & 91 & 118 \\
\hline & Trial 6 & 86 & 97 & 125 & 74 & 88 & 118 & 81 & 93 & 117 \\
\hline & Trial 7 & 73 & 84 & 107 & 72 & 86 & 120 & 79 & 90 & 112 \\
\hline
\end{tabular}




\begin{tabular}{|c|c|c|c|c|c|c|c|c|c|c|}
\hline & & \multicolumn{3}{|c|}{ MAX. Slope } & \multicolumn{3}{|c|}{ MAA } & \multicolumn{3}{|c|}{ OMRON } \\
\hline & & DBP & MAP & SBP & DBP & MAP & SBP & DBP & MAP & SBP \\
\hline \multirow{8}{*}{ 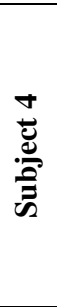 } & Trial 8 & 72 & 86 & 110 & 70 & 88 & 116 & 78 & 90 & 115 \\
\hline & Trial 9 & 84 & 96 & 122 & 74 & 87 & 114 & 79 & 92 & 119 \\
\hline & Trial 10 & 80 & 91 & 116 & 72 & 84 & 114 & 84 & 96 & 119 \\
\hline & Trial 11 & 80 & 92 & 112 & 73 & 89 & 117 & 80 & 90 & 111 \\
\hline & Trial 12 & 79 & 92 & 115 & 70 & 84 & 114 & 76 & 89 & 115 \\
\hline & Trial 13 & 71 & 85 & 115 & 70 & 82 & 107 & 69 & 83 & 112 \\
\hline & Trial 14 & 68 & 84 & 107 & 70 & 85 & 112 & 71 & 84 & 111 \\
\hline & Trial 15 & 70 & 90 & 114 & 69 & 85 & 116 & 73 & 87 & 116 \\
\hline \multirow{15}{*}{ 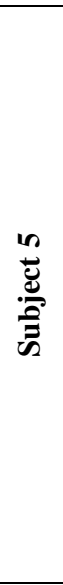 } & Trial 1 & 76 & 88 & 113 & 79 & 93 & 116 & 72 & 84 & 109 \\
\hline & Trial 2 & 71 & 84 & 108 & 75 & 85 & 110 & 69 & 81 & 106 \\
\hline & Trial 3 & 66 & 79 & 104 & 78 & 90 & 120 & 71 & 83 & 108 \\
\hline & Trial 4 & 73 & 85 & 114 & 79 & 91 & 115 & 68 & 78 & 99 \\
\hline & Trial 5 & 74 & 82 & 107 & 79 & 93 & 116 & 70 & 82 & 105 \\
\hline & Trial 6 & 70 & 91 & 116 & 81 & 92 & 114 & 75 & 88 & 113 \\
\hline & Trial 7 & 69 & 81 & 107 & 80 & 92 & 117 & 76 & 90 & 118 \\
\hline & Trial 8 & 69 & 81 & 107 & 78 & 91 & 114 & 73 & 87 & 115 \\
\hline & Trial 9 & 70 & 84 & 115 & 79 & 90 & 113 & 73 & 88 & 118 \\
\hline & Trial 10 & 68 & 80 & 109 & 78 & 89 & 113 & 73 & 87 & 114 \\
\hline & Trial 11 & 71 & 84 & 110 & 76 & 87 & 110 & 73 & 86 & 111 \\
\hline & Trial 12 & 79 & 91 & 121 & 80 & 91 & 116 & 74 & 88 & 117 \\
\hline & Trial 13 & 80 & 92 & 114 & 81 & 93 & 118 & 77 & 88 & 109 \\
\hline & Trial 14 & 76 & 85 & 107 & 79 & 91 & 117 & 73 & 83 & 104 \\
\hline & Trial 15 & 71 & 84 & 101 & 79 & 90 & 115 & 79 & 88 & 105 \\
\hline \multirow{15}{*}{ 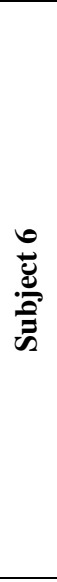 } & Trial 1 & 70 & 87 & 125 & 70 & 84 & 108 & 68 & 84 & 117 \\
\hline & Trial 2 & 69 & 83 & 111 & 68 & 82 & 107 & 68 & 82 & 109 \\
\hline & Trial 3 & 66 & 77 & 101 & 71 & 85 & 110 & 67 & 79 & 104 \\
\hline & Trial 4 & 67 & 81 & 113 & 70 & 84 & 106 & 62 & 78 & 110 \\
\hline & Trial 5 & 76 & 88 & 109 & 70 & 84 & 108 & 73 & 84 & 105 \\
\hline & Trial 6 & 75 & 90 & 121 & 70 & 84 & 106 & 70 & 86 & 118 \\
\hline & Trial 7 & 67 & 82 & 114 & 69 & 82 & 115 & 71 & 87 & 119 \\
\hline & Trial 8 & 74 & 90 & 122 & 67 & 82 & 113 & 70 & 86 & 119 \\
\hline & Trial 9 & 76 & 93 & 118 & 67 & 83 & 110 & 71 & 85 & 113 \\
\hline & Trial 10 & 75 & 91 & 124 & 67 & 82 & 107 & 73 & 89 & 120 \\
\hline & Trial 11 & 68 & 81 & 107 & 70 & 84 & 110 & 72 & 85 & 112 \\
\hline & Trial 12 & 69 & 86 & 120 & 70 & 86 & 110 & 65 & 82 & 116 \\
\hline & Trial 13 & 70 & 84 & 111 & 68 & 82 & 107 & 66 & 80 & 107 \\
\hline & Trial 14 & 68 & 82 & 109 & 66 & 80 & 104 & 66 & 80 & 107 \\
\hline & Trial 15 & 60 & 74 & 107 & 69 & 82 & 107 & 65 & 81 & 114 \\
\hline \multirow{10}{*}{ 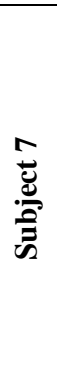 } & Trial 1 & 81 & 82 & 114 & 84 & 94 & 122 & 85 & 99 & 127 \\
\hline & Trial 2 & 86 & 97 & 127 & 86 & 91 & 118 & 82 & 95 & 121 \\
\hline & Trial 3 & 83 & 94 & 118 & 88 & 90 & 108 & 81 & 92 & 116 \\
\hline & Trial 4 & 81 & 92 & 115 & 70 & 96 & 108 & 78 & 89 & 111 \\
\hline & Trial 5 & 82 & 94 & 120 & 75 & 86 & 125 & 79 & 92 & 119 \\
\hline & Trial 6 & 70 & 82 & 105 & 78 & 83 & 113 & 78 & 89 & 113 \\
\hline & Trial 7 & 71 & 85 & 114 & 78 & 96 & 116 & 75 & 89 & 118 \\
\hline & Trial 8 & 83 & 91 & 116 & 77 & 96 & 110 & 77 & 89 & 114 \\
\hline & Trial 9 & 82 & 93 & 117 & 77 & 95 & 108 & 77 & 89 & 115 \\
\hline & Trial 10 & 82 & 94 & 116 & 80 & 90 & 109 & 82 & 93 & 115 \\
\hline \multirow{7}{*}{ 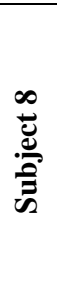 } & Trial 1 & 86 & 95 & 119 & 80 & 90 & 110 & 82 & 93 & 116 \\
\hline & Trial 2 & 80 & 89 & 110 & 79 & 88 & 115 & 85 & 95 & 117 \\
\hline & Trial 3 & 76 & 81 & 97 & 86 & 87 & 101 & 80 & 87 & 102 \\
\hline & Trial 4 & 77 & 85 & 106 & 86 & 79 & 100 & 80 & 89 & 107 \\
\hline & Trial 5 & 74 & 81 & 99 & 74 & 80 & 99 & 76 & 84 & 102 \\
\hline & Trial 6 & 78 & 84 & 94 & 81 & 93 & 104 & 82 & 88 & 100 \\
\hline & Trial 7 & 87 & 94 & 118 & 80 & 96 & 115 & 82 & 92 & 113 \\
\hline
\end{tabular}




\begin{tabular}{|c|c|c|c|c|c|c|c|c|c|c|}
\hline & & \multicolumn{3}{|c|}{ MAX. Slope } & \multicolumn{3}{|c|}{ MAA } & \multicolumn{3}{|c|}{ OMRON } \\
\hline & & DBP & MAP & SBP & DBP & MAP & SBP & DBP & MAP & SBP \\
\hline & Trial 8 & 86 & 97 & 114 & 80 & 85 & 112 & 83 & 92 & 110 \\
\hline & Trial 9 & 74 & 84 & 109 & 86 & 88 & 115 & 80 & 91 & 115 \\
\hline & Trial 10 & 80 & 89 & 111 & 79 & 96 & 107 & 84 & 93 & 111 \\
\hline \multirow{10}{*}{ 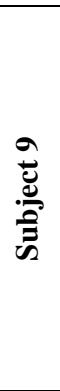 } & Trial 1 & 69 & 81 & 109 & 90 & 89 & 112 & 65 & 78 & 104 \\
\hline & Trial 2 & 65 & 76 & 100 & 62 & 75 & 98 & 60 & 71 & 94 \\
\hline & Trial 3 & 69 & 78 & 104 & 66 & 71 & 102 & 62 & 74 & 99 \\
\hline & Trial 4 & 71 & 80 & 105 & 66 & 72 & 105 & 64 & 76 & 100 \\
\hline & Trial 5 & 56 & 69 & 91 & 65 & 69 & 94 & 66 & 75 & 94 \\
\hline & Trial 6 & 75 & 82 & 103 & 73 & 74 & 96 & 69 & 79 & 99 \\
\hline & Trial 7 & 73 & 81 & 104 & 71 & 74 & 96 & 65 & 76 & 99 \\
\hline & Trial 8 & 71 & 82 & 107 & 70 & 73 & 109 & 64 & 77 & 103 \\
\hline & Trial 9 & 71 & 84 & 109 & 69 & 72 & 110 & 65 & 77 & 102 \\
\hline & Trial 10 & 70 & 77 & 107 & 67 & 70 & 112 & 64 & 74 & 94 \\
\hline \multirow{10}{*}{ 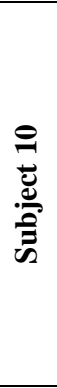 } & Trial 1 & 79 & 87 & 109 & 77 & 89 & 110 & 71 & 82 & 104 \\
\hline & Trial 2 & 71 & 82 & 107 & 73 & 74 & 112 & 65 & 78 & 104 \\
\hline & Trial 3 & 78 & 94 & 119 & 71 & 84 & 118 & 70 & 84 & 113 \\
\hline & Trial 4 & 68 & 77 & 94 & 70 & 77 & 108 & 71 & 80 & 98 \\
\hline & Trial 5 & 68 & 78 & 105 & 70 & 85 & 101 & 71 & 83 & 107 \\
\hline & Trial 6 & 71 & 79 & 114 & 69 & 79 & 96 & 63 & 75 & 101 \\
\hline & Trial 7 & 70 & 83 & 105 & 68 & 83 & 98 & 70 & 81 & 105 \\
\hline & Trial 8 & 70 & 79 & 109 & 69 & 79 & 101 & 66 & 77 & 101 \\
\hline & Trial 9 & 59 & 70 & 96 & 61 & 77 & 104 & 63 & 76 & 103 \\
\hline & Trial 10 & 62 & 75 & 101 & 63 & 79 & 109 & 67 & 80 & 107 \\
\hline \multirow{10}{*}{ 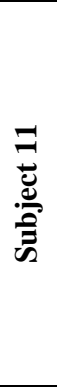 } & Trial 1 & 72 & 80 & 103 & 69 & 71 & 95 & 64 & 74 & 96 \\
\hline & Trial 2 & 57 & 66 & 86 & 55 & 74 & 93 & 59 & 69 & 89 \\
\hline & Trial 3 & 64 & 75 & 98 & 70 & 69 & 92 & 62 & 73 & 95 \\
\hline & Trial 4 & 55 & 64 & 88 & 70 & 79 & 89 & 57 & 68 & 91 \\
\hline & Trial 5 & 66 & 77 & 99 & 71 & 81 & 96 & 63 & 73 & 94 \\
\hline & Trial 6 & 62 & 71 & 93 & 58 & 75 & 91 & 56 & 66 & 88 \\
\hline & Trial 7 & 61 & 69 & 90 & 59 & 74 & 88 & 58 & 66 & 84 \\
\hline & Trial 8 & 59 & 70 & 86 & 55 & 69 & 85 & 55 & 64 & 82 \\
\hline & Trial 9 & 58 & 66 & 86 & 54 & 66 & 88 & 57 & 66 & 86 \\
\hline & Trial 10 & 64 & 71 & 89 & 66 & 64 & 86 & 57 & 65 & 83 \\
\hline \multirow{5}{*}{ 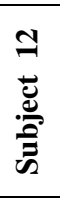 } & Trial 1 & 66 & 79 & 104 & 59 & 79 & 110 & 64 & 76 & 102 \\
\hline & Trial 2 & 62 & 76 & 102 & 61 & 70 & 98 & 58 & 70 & 96 \\
\hline & Trial 3 & 60 & 73 & 94 & 69 & 77 & 98 & 62 & 75 & 101 \\
\hline & Trial 4 & 55 & 68 & 90 & 64 & 76 & 105 & 57 & 71 & 99 \\
\hline & Trial 5 & 69 & 84 & 115 & 69 & 70 & 110 & 62 & 78 & 110 \\
\hline \multirow{5}{*}{ 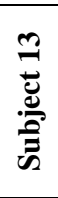 } & Trial 1 & 73 & 86 & 114 & 69 & 78 & 115 & 68 & 82 & 111 \\
\hline & Trial 2 & 71 & 88 & 118 & 72 & 86 & 119 & 70 & 84 & 114 \\
\hline & Trial 3 & 71 & 85 & 113 & 72 & 83 & 108 & 70 & 83 & 111 \\
\hline & Trial 4 & 79 & 90 & 115 & 69 & 83 & 101 & 71 & 83 & 109 \\
\hline & Trial 5 & 80 & 91 & 117 & 71 & 89 & 119 & 73 & 86 & 113 \\
\hline \multirow{5}{*}{ 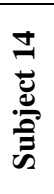 } & Trial 1 & 71 & 84 & 122 & 79 & 95 & 125 & 80 & 93 & 119 \\
\hline & Trial 2 & 70 & 81 & 115 & 79 & 96 & 122 & 80 & 93 & 120 \\
\hline & Trial 3 & 84 & 97 & 128 & 69 & 96 & 126 & 78 & 93 & 124 \\
\hline & Trial 4 & 80 & 94 & 124 & 65 & 95 & 122 & 79 & 92 & 120 \\
\hline & Trial 5 & 74 & 89 & 114 & 71 & 89 & 119 & 78 & 91 & 118 \\
\hline \multirow{5}{*}{ 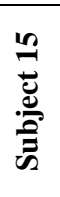 } & Trial 1 & 55 & 67 & 93 & 69 & 70 & 91 & 61 & 72 & 95 \\
\hline & Trial 2 & 64 & 75 & 98 & 69 & 70 & 96 & 61 & 72 & 94 \\
\hline & Trial 3 & 61 & 72 & 96 & 64 & 68 & 90 & 58 & 69 & 92 \\
\hline & Trial 4 & 67 & 78 & 100 & 58 & 69 & 90 & 60 & 70 & 90 \\
\hline & Trial 5 & 66 & 73 & 89 & 60 & 64 & 89 & 62 & 69 & 85 \\
\hline
\end{tabular}




\begin{tabular}{|c|c|c|c|c|c|c|c|c|c|c|}
\hline & & \multicolumn{3}{|c|}{ MAX. Slope } & \multicolumn{3}{|c|}{ MAA } & \multicolumn{3}{|c|}{ OMRON } \\
\hline & & DBP & MAP & SBP & DBP & MAP & SBP & DBP & MAP & SBP \\
\hline \multirow{5}{*}{ 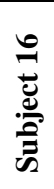 } & Trial 1 & 71 & 85 & 117 & 70 & 90 & 125 & 78 & 92 & 120 \\
\hline & Trial 2 & 70 & 82 & 112 & 71 & 94 & 122 & 76 & 88 & 114 \\
\hline & Trial 3 & 76 & 90 & 117 & 76 & 88 & 119 & 73 & 87 & 115 \\
\hline & Trial 4 & 80 & 91 & 115 & 74 & 82 & 109 & 77 & 88 & 111 \\
\hline & Trial 5 & 79 & 88 & 108 & 62 & 81 & 106 & 72 & 83 & 105 \\
\hline \multirow{5}{*}{ 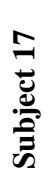 } & Trial 1 & 79 & 93 & 124 & 77 & 93 & 120 & 76 & 91 & 122 \\
\hline & Trial 2 & 80 & 96 & 127 & 79 & 89 & 125 & 77 & 92 & 123 \\
\hline & Trial 3 & 80 & 87 & 104 & 69 & 86 & 95 & 74 & 81 & 97 \\
\hline & Trial 4 & 79 & 88 & 101 & 80 & 88 & 96 & 83 & 90 & 105 \\
\hline & Trial 5 & 74 & 82 & 107 & 79 & 81 & 115 & 77 & 88 & 112 \\
\hline \multirow{5}{*}{ 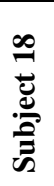 } & Trial 1 & 72 & 87 & 117 & 69 & 81 & 115 & 70 & 84 & 113 \\
\hline & Trial 2 & 77 & 93 & 122 & 77 & 87 & 122 & 77 & 91 & 120 \\
\hline & Trial 3 & 67 & 82 & 111 & 68 & 82 & 119 & 70 & 84 & 114 \\
\hline & Trial 4 & 75 & 88 & 118 & 69 & 84 & 116 & 72 & 86 & 115 \\
\hline & Trial 5 & 79 & 87 & 115 & 69 & 85 & 112 & 72 & 85 & 112 \\
\hline
\end{tabular}




\section{APPENDIX C - Informed Consent Form}

\section{Informed Consent Form}

\section{Blood Pressure Measurement in Healthy Subjects}

The research project is being conducted by professors Hilmi Dajani, Voicu Groza, and Miodrag Bolic at the School of Information Technology and Engineering of the University of Ottawa. (Tel. (613) 5625800, ext. 6217).

Purpose: This informed consent form is to make sure that you understand the nature of your involvement in this study, and to obtain your informed consent to participate in this study.

Procedure: You will be comfortably seated on a chair, and asked to stay relaxed with minimum movement. You might be asked to breath at a specified rhythm for a few minutes. A cuff will be placed on your arm, a wristband on the opposite wrist and possibly a belt on your chest to take measurements. The entire recording session will last up to 60 minutes. You will be asked to participate on 5 different days ( 1 session /day).

Subject description: Healthy adults with no known history of heart disease will be included.

Risks to participating: There is no danger or risk to health associated with this study. All procedures have been pre-tested and they have been used routinely for many years in hospitals, clinics, and laboratories.

Withdrawing from the study: Your participation in this study is voluntary. You may withdraw from the study at any time, by verbally informing the investigator or any of the researchers, even after signing the form. There will be no consequences following this action.

If you have any concerns with regards to the ethical conduct of the study, you may contact the Protocol Officer for Ethic in Research, University of Ottawa, Tabaret Hall, 550 Cumberland Street, Room 159, 
Compensation: You will not receive monetary compensation for this study.

Confidentiality: Any information about you collected during the study will be kept strictly confidential. Your name will not be associated with the collected data in any way. While the results will appear in student's dissertations and may also be published, you will not be identified.

The data collection will be conducted by Dr. Dajani, his graduate students or research fellows, or his research assistant. The records will be kept on computer files. The files will be password-protected. The data will be kept in Dr. Dajani's office when not in use and will be conserved for a maximum period of 10 years, after which the computer files will be deleted.

In closing: With your participation, you will be given a copy of this consent form. At the conclusion of the study, should you wish, you will be provided with a summary of the results. You may ask questions at any time, even after signing this consent form.

Signatures: I have read the above description of the study and understand the conditions of participation. My signature indicates that I agree to participate in the study.

Please indicate if you want to receive a summary of your results (please write YES or NO):

Name of participant (please print name here):

\section{Participant's Signature:}

\section{Date:}

\section{Researcher's Signature:}

Date: 


\section{References:}

[1] H. Sorvoja and R. Myllyla, " noninvasive blood pressure measurement methods," molecular and quantum acoustic, vol. 27,pp. 239-267. 2006.

[2]S. Fox, Human Physiology, 8th ed. New York: McGraw-Hill, 2007.

[3] J. G. Wbster “ Medical Instrumentation, Application and Design” USA, CANADA: John Wiley \& Sons, INC. 1998.

[4] W.A. Littler, B. Komosuoglu, "Which is the Most Accurate Method of Measuring Blood Pressure?," American Heart Journal, vol. 117, Mar. 1989, pp. 723-728.

[5] K. Turner, Arterial Blood Pressure Monitoring: An introduction. St Vincent's Hospital, Intensive Theraphy Unit, USA, 2000.

[6] X. F. Teng, and Y. T. Zhang, "Continous and Noninvasive estimation of Arterial Blood Pressure Using a Photoplethysmographic Approach,” IEEE EMBS, Cancun, Mexico, 2003.

[7] Z. B. Zhang, and T. H. Wu, “ The Technique of Non-Invasive Blood Pressure Measurement and its Development," Chinese Journal of Medical Instrumentation, Vol.27, pp. 196-199, 2003.

[8] P.C. Wankum, T.L. Thurman, S.J. Holt, R. A. Hall, P. M. Simpson, and M. J. Heulitt, "Validation of a Noninvasive Blood Pressure Monitoring Device in Normotensive and Hypertensive Pediatric Intensive Care Patients," J. Clinical Monitoring and Computing, Vol. 18, pp. 253-263, 2005.

[9] L. A. Geddes, "Handbook of blood Pressure Measurement," Humana Press, 1991.

[10] D. J. Sebald, D. E. Bahr, and A. R. Kahn, "Narrowband ascultatory blood pressure measurement," IEEE Trans. Biomed. Eng. Vol. 49, no. 9, pp. 1038-1044, 2002.

[11] N.M. Noaman, and A. K. Abbas, " system identification of integrative non invasive blood pressure sensor based on ARMAX estimator algorithm," Medicon 2007, IFMBE Proceedings 16, pp. 385-389, 2007.

[12] K.G. Ng, C.F. Small, "Survey of Automated Noninvasive Blood Pressure Monitors," Journal of Clinical Engineering, Vol. 33, Dec. 1994, pp. 452-475.

[13] E.J. Marey, Ecole Practique des Hautes Etudes, Physiologie Experimentale, vol. 6, 1876, pp. 307-343. 
[14] S.C Millasseau, R.P. Kelly, J.M. Ritter, and P.J. Chowienczyk "Determination of agerelated increase in lare artery stiffness by digital pulse contour analysis," Clinical Science, Vol. 103, pp.371-377, 2002.

[15] T. Ellis, J. McNames, and M. Aboy, "Pulse Morphology Visualization and Analysis With Application in Cardiovascular Pressure Signals," IEEE Transaction on Biomedical Engineering, Vol. 54, No. 9 pp 1552-1559, September 2007.

[16] D. Korpas, J. Halek, and L. Dolezal, “ Parameters Describing the Pulse Wave,” Physiol. Res. Vol. 58, pp. 473-479, 2009.

[17] S. Omboni, G. Parai, A. Frattola, E. Mutti, M. Di Rienzo, P. Castiglioni, and G. Mancia, "Spectral and sequence analysis of finger blood pressure variability. Comparison with analysis of intra-arterial recording," Hypertension, Vol. 22, pp. 26-33.

[18] V.K. Jayasree, T.V. Sandhya, P. Radhakrishnan, "Non-invasive Studies on Age Related Parameters Using a Blood Volume Pulse Sensor," Measurement Science Review, Vol. 8, Section 2, No. 4, pp. 82-86, 2008.

[19] J.R. Jago, and A. Murray, "Repeatability of peripheral pulse measurement on ears, fingers, and toes using photoelectric plethysmography," Clin. Phys. Physiol. Meas. Vol. 9. pp. 319329. 1988.

[20] R. Kelly, C. Hayward, A. Avolio, and M. O’Rourke, 'Noninvasive determination of agerelated changes in the human arterial pulse," Circulation, Vol. 80 pp. 1652-1659, 1989.

[21] G. E. McVeigh, C. W. Bratteli, D. J. Morgan, C. M. Alinder, S. P. Glasser, S. M. Finkelstein, and J. N. Cohn, “ Age-Related Abnormalities in Arterial Compliance Identified by Pressure Pulse Contour Analysis: Aging and Arterial Compliance," Hypertension, Vol. 33 pp. 1392-1398, 1999.

[22] E. D. Freis, W. C. Heath, P. C. Lunchsinger, and R. E. Snell, "Changes in the carotid pulse which occur with age and hypertension," AM. Heart A., Vol. 71, Num. 6, pp. 757-765, 1966.

[23] S. C. Millasseau, R. P. Kelly, J. M. Ritter, and P.J. Chowienczyk, "Determination of agerelated increase in large artery stiffness by digital pulse contour analysis," Clinical Science, Vol. 103 pp. 371-377, 2002. 
[24] J. Allen, and A. Murray, "Age-related Changes in the characteristics of the pholoplethysmographic pulse shape at various body sites," Physiological Measurement, Vol. 24, pp. 297-307, 2003.

[25] J. Allen, A. Murray, "Age-Related changes in peripheral pulse timing characteristics at the ear, fingers, and toe," J. Hum. Hypertens, Vol. 16, pp. 711-717, 2002.

[26] J. Allen, "Photoplethysmography and its application in clinical physiological measurement," Physiol. Meas. Vol. 28 pp. R1-R39, 2007.

[27] E. D. Lehmann, “Clinical value of aortic pulse-wave velocity measurement," Lancet, Vol. 354 No. 9178 pp. 528-529, 1999.

[28] A. Yamashina, H. Tomiyama, K. Takeda, H. Tsuda, T. Arai, and K. Hirose, "Validity, reproducibility, and clinical significant of noninvasive brachial ankle pulse wave velocity measurement," Hypertens. Res. Vol. 25 No. 3 pp. 359-364, 2002.

[29] R. Asmar, A. Benetos, J. Topoucluan, P. Laurent, B. Pannier, and A. M. Brisac, “Assesment of arterial dispensability by automatic pulse wave velocity measurement, validation and clinical application studies," Hypertension, Vol. 26, No. 3, pp. 485-490, 1995.

[30] J.M. Padilla, E.J. Berjano, U. Saiz, L. Facila, P. Diaz, and S. Merce, "Assesment of Relationships between Blood Pressure, Pulse Wave Velocity and Digital Volume Pulse," Computers in Cardiology, Vol. 33, pp. 893-896, 2006.

[31] S. Laurent, P. Boutouyrie, R. Asmar, I. Gautier, B. Laloux, and L. Guize, "Aortic Stiffness is an independent predictor of all-cause and cardiovascular mortality in hypertensive patients," Hypertension, Vol. 37, No. 5, pp. 1236-1241, 2001.

[32] Y. Matsui, K. Kario, J. Ishikawa, K. Eguchi, S. Hoshida, and K. Shimada, "Reproducibility of arterial stiffness indices (pulse wave velocity and augmentation Index) simultaneously assessed by automated pulse wave analysis and their associated risk factors in essential hypertensive patients," Hypertens. Res. Vol. 27 No. 11, pp. 851-857, 2004.

[33] B. Williams, P. S. Lacy, S. M. Thom, K. Cruichshank, A. Stanton, D. Collier, A. D. Hughes, H. Thurston, "Differential Impact of Blood Pressure-Lowering Drugs on Central Aortic Pressure and Clinical Outcomes," Circulation, Vol. 113, pp. 1213-1225, 2006. 
[34] R. Klocke, J. R. Cockcroft, and G. J. Taylor, "Arterial Stiffness and Central Blood Pressure, as determined by pulse wave analysis, in rheumatoid arthritis," Ann Rheum Dis, Vol. 62, pp. 414-418, 2003.

[35] I. B. Wilkinson, N. H. Mohammad, S. Tyrrell, I. R. Hall, D. J. Webb, V. E. Paul, T. Levy, and J. R. Cockcroft, “ Heart Rate Dependency of Pulse Pressure Amplification and Arterial Stiffness," AJH, Vol. 15, pp. 24-30, 2002.

[36] P. Boutouyrie, C. Bussy, P. Lacolley, X. Girerd, B. Laloux, and S. Laurent, "Association Between Local Pulse Pressure, Mean Blood Pressure, and Large-Artery Remodeling," Circulation, Vol. 100, pp. 1387-1393, 1999.

[37] G. Gelido, S. Angiletta, A. Pujalte, P. Quiroga, P. Cornes, and D. Craiem, “Arterial pressure measurement: Is the envelop curve of the oscillometric method influenced by arterial stiffness?," Journal of Physics, Conference Series 90, 2007.

[38] M. Nitzan, B. Khanokh, and Y. Slovik, " the difference in pulse transit time to the toe and finger measured by photoplethysmography," Physiol. Meas. Vol. 23, pp. 85-93, 2002.

[39] J. S. Kim, K. K. Kim, H. J. Baek, and K. S. Parl, "Comparable Prameter related to arterial stiffness in Blood Pressure Estimation Method," IEEE International Conference on Information Technology and Application in Biomedicine, pp. 410-411, 2008.

[40] J.C. Bramwell, and A. V. Hill, "Velocity of Transmission of the pulse wave and elasticity of arteries," Lancent, Vol. 1. Pp. 891-892, 1922.

[41] M. Eliakim, D. Sapoznikov, amd J. Wienman, " Pulse wave velocity in healthy subjects and in patients with various diseases states," Am. Heart J. Vol. 82, pp. 448-457, 1971.

[42] M. F. O’Rourke, and D. E. Gallagher, "Pulse wave analysis," J. hypertens. Vol. 14, pp. 160167, 1993.

[43] N. Westerhof, and M. F. O'Rourke, "Haemodynamic basis for the development of left ventricle failure in systolic hypertension and for its logical treatment," J. Hypertens, Vol. 13, pp. 943-952, 1995.

[44] P. S. Saba, M. J. Pini, M. Spitzer, A. Ganau, and R. B. Devereux, "Relation of arterial pressure waveform to left ventricular and carotid anatomy in normotensive subjects," $J$. Am. Coll. Cardiol., Vol. 22, pp. 1873-1880, 1993. 
[45] E. G. Lakatta, "Similar myocardial effects of aging and hypertension," Eur Heart J. Vol. 11, pp. 29-38, 1991.

[46] L. Xu, Y. Wang, C. Feng, Y. Yao, and Y. Zhao, "Variability Analysis on the Parameters of Pulse Waveform,” Bioinformatics and Biomedical Engineering (ICBBE) , pp. 1-3, 2010.

[47] S. Ahmad, S. Chen, K. Soueidan, I. Batkin, M. Bolic, H. Dajani, V. Groza, "A Prototype of an Integrated Blood Pressure and Electrocardiogram Device for Multi-Parameter Physiologic Monitoring," IEEE International Instrumentation and Measurement Technology Conference I2MTC 2010, Austin, Texas, May 2010, pp. 1244-1249.

[48] M. Pagani, F. Lombardi, S. Guzzetti, O. Rimoldi, R. Furlan, P. Pizzinelli, G. Sandrone, G. Malfatto, S. Dell'Orto, E. Piccaluga, M. Turiel, G. Baselli, S. Cerutti, A. Malliani, "Power Spectral Analysis of Heart Rate and Arterial Pressure Variabilities as a Marker of Sympathovagal Interaction in Man and Conscious Dog," Circulation Research, Vol. 59, Aug. 1986, pp. 178-193.

[49] H. Sorvoja, and R. Myllyla, "Noninvasive blood pressure measurement methods,"Molecular and quantum acoustics, Vol. 27, pp. 239-264, 2006.

[50] D. Goldbring, and H. Wohltmann, "Flush Method for Blood Pressure Determination in Newborn Infants,” J. of Pediatrics, Vol. 40, pp.285-290, 1952.

[51] W. A. Littler, and B. Komsuoglu, "Which is the most accurate method of measuring blood pressure?," American Heart Journal, Vol. 117, no. 3, pp. 723-728, 1989.

[52] W. W. Nichols, and M. F. O’Rourke, "McDonald's Blood Flow in Arteris," 4th edition, 1998.

[53] G. Drzewiecki, J. Melbin, and A. Noordengraaf, "Arterial tonometry: review and analysis," J. Biomech. Vol. 16, pp. 141-152, 1983.

[54] E. A. Zorn, M. B. Wilson, J. Angel, J. Zanella, and B. S. Alpert, "Validation of an automated arterial tonometry monitor using Association for the Advancement of Medical Instrumentation Standards,” Blood Press. Monitor. Vol.2, no. 4, pp. 185-188, 1997.

[55] L. A. Geddes, M. Voelz, C. Combs, D. Reiner, C. F. Babbs, "Characterization of the Oscillometric Method for Measuring Indirect Blood Pressure," Annals of Biomedical Engineering, Vol. 10, pp. 271-280, 1982. 
[56] T. K. Lee, and D. R. Westenkow, "Comparison of Blood Pressure Measured by Oscillometry from the Supraorbital Artery and Invasively from the Radial Artery," Journal of Clinical Monitoring and Computing, Vol. 14, No. 2, pp. 113-117, 1998.

[57] J. V. T. B. Moraes, M. Cerulli, and P.S. Ng "A Strategy for Determination of Systolic, Mean and Diastolic Blood Pressures from Oscillometric Pulse Profiles," Computers in Cardiology, Vol. 27, pp. 211-214, 2002.

[58] J.C.T.B. Moraes, M. Cerulli, P.S. Ng, "Development of a New Oscillometric Blood Pressure Measurement System," IEEE Computers and Cardiology, Hannover, Germany, Sep. 1999, pp. 467-470.

[59] J.N. Amoore, "Extracting Oscillometric Pulses from the Cuff Pressure: Does it Affect the Pressures Determined by Oscillometric Blood Pressure Monitors?," Blood Pressure Monitoring, Vol. 11, Oct. 2006, pp. 269-279.

[60] H. Sorvoja, R. Myllyla, P. Karja-Koskenkari, J. Koskenkari, M. Lilja, A. Kesaniemi, "Accuracy Comparison of Oscillometric and Electronic Palpation Blood Pressure Measuring Methods Using Intra-Arterial Method as a Reference," Molecular and Quantum Acoustics, Vol. 26, 2005, pp. 235-260.

[61] A. Ball-llovera, R. Del Rey, R. Ruso, J. Ramos, O. batista, and I. Niubo, “ An Experience in Implementing the Oscillometric Algorithm for the Non-Invasive Determination of Human Blood Pressure," Annual International Comnference of the IEEE EMBS, Vol. 4, pp. 3173$3175,2003$.

[62] S. Chen, V. Z. Groza, M. Bolic, H. R. Dajani, "Assesment of Algorithms for Oscillometric Blood Pressure Measurement," International Instrumentation and Measurement Technology Conferences, pp.1763-1767, 2009.

[63] D. B. McCombie, A. T. Reisner, H. H. Asada, and P. Shaltis, Patent No. US 2008/0039731 A1. Boston/USA, 2008.

[64] M. Mafi, M. Bolic, V. Z. Groza, H. Dajani, S. Rajan, "Oscillometric Blood Pressure Pulse Morphology," MeMeA 2011, [submitted on 15/April/2011].

[65] M. Mafi, S. Rajan, M. Bolic, V. Z. Groza, H. Dajani, “ Blood Pressure Estimation using Oscillometric Pulse Morphology,” IEEE EMBS, [submitted on 20/June/ 2011]. 
[66] G. Drzewiecki, R. Hood, and H. Apple, Theory of the Oscillometric Maximum and the systolic and Diastolic Detection Ratios," Annals of Biomedical Engineering, Vol. 22, pp. 8896, 1994.

[67] AAMI/ANSI-SP10, Manual, Electronic or Automated Sphygmomanometers. ANSI ( Americal National Standars Institude), AAMI (Association for the advancement of Medical Instrumentation), Arlington, 2002.

[68] S. Hales, Statistical Essays, vol. 2, London, W. Innys, 1733.

[69] S. Iamratanakul, J. McNames, B. Goldstein, "Estimation of Respiration from Physiologic Pressure Signals," Proceedings of the $25^{\text {th }}$ Annual International Conference of the IEEE Engineering in Medicine and Biology Society, Cancun, Mexico, Sep. 2003, pp. 2734-2737.

[70] S. Bruno, P. Scalart, "Estimation of Cardiac and Respiratory Rhythms Based on an AMFM Demodulation and an Adaptive Eigenvector Decomposition," $13^{\text {th }}$ European Signal Processing Conference, Antalya, Turkey, Sep. 2005.

[71] M. Ramsey III, "Blood Pressure Monitoring: Automated Oscillometric Devices,” Journal of Clinical Monitoring and Computing, Vol. 7, Jan. 1991, pp. 55-67.

[72] Doohan, James (1999, Sept. 17), "Lesson 4: Circulatory System” in BioMed 108 - Human Physiology, [Online]. Available:

http://www.biosbcc.net/doohan/sample/htm/COandMAPhtm.htm.

[73] G.G. Bernstson, J.T. Cacioppo, K.S. Quigley, "Respiratory Sinus Arrhythmia: Autonomic Origins, Physiological Mechanisms and Psychophysiological Implication," Psychophysiology, vol. 30, Mar. 1993, pp. 183-196.

[74] C.P.M Leeson, P.H. Whincup, D.G. Cook, M.J. Mullen, A.E. Donald, C. A. Seymour, J.E. Deanfield, " Cholestrol and Arterial Distensibility in the First Decade of Life," Circulation, Vol.101, pp. 1533-1538, 2000.

[75] T.G. Pickering, J. E. Hall, L. J. Appel, Bonita. E. Falkner, J. Graves, M. N. Hill, D. W. Jones, T. Kurtz, S. G. Sheps, and E. J. Roccella, “ Recommendations for Blood Pressure Measurement in Humans and Experimental Animals: Part 1: Blood Pressure Measurement in in Humans: A Statement for Progessionals From the Subcommittee of Professional and Public Education of the American Heart Association Council on High Blood Pressure Research," Hypertension, Vol. 45, pp. 142-161, 2005. 
[76] P. Tozzi, A. Corno, and D. Hayoz, "Definition of arterial compliance," AM J Physiol Heart Circ. Physiol, Vol. 278, pp. 1407, 2000.

[77] A.C. Dornhost, P. Howard, G.L. Leathart, "Respiratory Variations in Blood Pressure," Circulation, Vol. 6, pp. 553-558, 1952.

[78] L. Suganthi, M. Manivannan, “ Effect of upper arm cuff pressure on pulse morphology using photoplethysmography," Conf. Proc. IEEE Eng. Med. Biol. Soc., Vol. 5, pp. 17921794, 2009. 